\title{
GENETIC AND MICROBIOTA-MEDIATED PHENOTYPE VARIABILITY IN THE MIN MOUSE MODEL OF HUMAN COLORECTAL CANCER
}

\author{
A Dissertation \\ presented to \\ the Faculty of the Graduate School \\ at the University of Missouri-Columbia \\ In Partial Fulfillment \\ of the Requirements for the Degree \\ Doctor of Philosophy \\ by \\ JACOB E. MOSKOWITZ \\ Dr. James Amos-Landgraf, Dissertation Supervisor \\ JULY 2019
}


(C) Copyright by Jacob E. Moskowitz 2019

All Rights Reserved 
The undersigned, appointed by the dean of the Graduate School, have examined the dissertation entitled

\section{GENETIC AND MICROBIOTA-MEDIATED PHENOTYPE VARIABILITY IN THE MIN MOUSE MODEL OF HUMAN COLORECTAL CANCER}

presented by Jacob E. Moskowitz, a candidate for the degree of doctor of philosophy and hereby certify that, in their opinion, it is worthy of acceptance.

Dr. James Amos-Landgraf

Dr. Jeffrey Bryan

Dr. Aaron Ericsson

Dr. Craig Franklin

Dr. Lloyd W. Sumner 


\section{ACKNOWLEDGEMENTS}

I would like to express my deepest gratitude to my advisor, Dr. James AmosLandgraf, who has consistently encouraged me throughout the circuits of graduate training. Your ability to promote independence and critical thinking while providing a safety net in times of sincere need is a unique attribute that I value highly in your mentorship. I always appreciate your worldly outlook on life, highlighted by your numerous hidden facts about geography and culture across the globe. You have been a selfless advocate for my continued growth and professional development, and you have certainly transformed me into a better scientist, thinker, and overall person.

I would also like to thank Dr. Craig Franklin, who has been an integral part of my mentorship throughout both veterinary school and laboratory animal residency. True to your generous form, you have consistently supported me since I first expressed interest in research and laboratory animal medicine. You have unfailingly been in my corner through every challenge faced, and constantly remind me that life is an adventure to experience to the fullest. Your friendship and guidance have been invaluable.

Many thanks to my committee members, Dr. Craig Franklin, Dr. Aaron Ericsson, Dr. Lloyd W. Sumner, and Dr. Jeffrey Bryan. Each of you has provided a fantastic outlook on different ways to think about and approach science. Your thought-provoking questions and helpful suggestions have been critical to pushing me forward throughout my training. I can only hope that as I continue to progress, I have the opportunity to model your mentorship styles to help future generations of scientists as you have helped me. 
I would also like to thank my former lab colleague, Dr. Susheel Busi, for his guidance during our time together in the Amos-Landgraf lab. As a new graduate student, you were endlessly patient in teaching me new techniques and analyses that would later become vital to my success. I would certainly be lost in the depths of microbiome analysis and multivariate statistics without your mentorship. Beyond that, our unplanned conversations about the complexities of science always helped me put things into perspective, and to remind me that science is not supposed to be easy.

The support of the Comparative Medicine Program has been central to my graduate education. To all faculty, staff, and trainees of the CMP, thank you for your flexibility in allowing me to pursue my many scientific endeavors. Each of your unique skillsets, and your willingness to share them with me, amazes me. Special thanks to Shelly Nail for helping me navigate the difficulties of this journey. For every supplyorder error you helped me circumvent, every flight you booked for conference travel, and most importantly for every time you went the extra mile to make a welcoming presence, thank you. I would also like to thank all members of Discovery Ridge and IDEXX Bioresearch for their contributions to my education and personal growth throughout my years here.

No mention of my journey would be complete without acknowledgment of my mother, Ruth Fisher. Your unwavering support through times of great change in my life have made each success a possibility. Whether it is my academic pursuits, or standing idly by as I traipse into an unknown wilderness, you accept my adventurous and independent spirit. Knowing you are in my corner no matter the circumstances continues to give me the confidence and drive to pursue my passions. 


\section{TABLE OF CONTENTS}

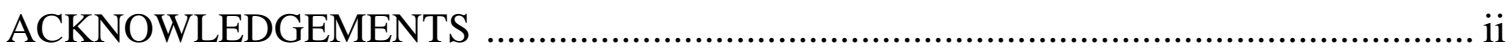

LIST OF ABBREVIATIONS ............................................................................... viii

LIST OF FIGURES

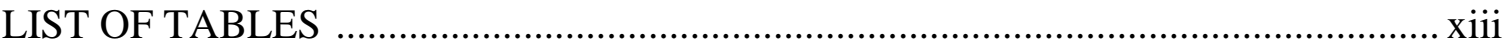

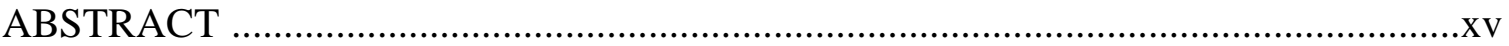

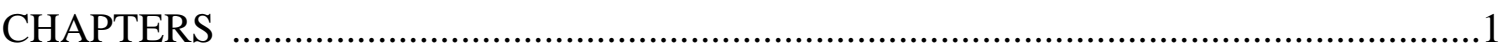

I. An Overview of Genetic and Microbial Modifiers of Colorectal Cancer

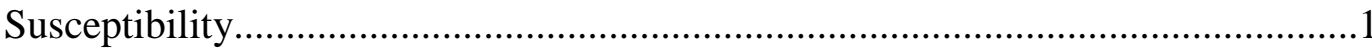

1.1 Significance and overview of colorectal cancer ...........................................

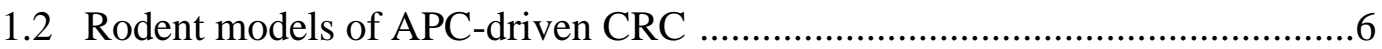

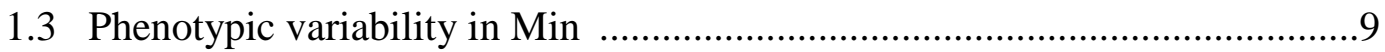

1.4 The microbiome in CRC ………….........................................................13

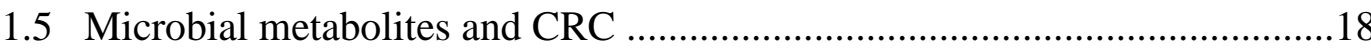

1.6 From characterization to function: tools to assess host-GM interactions .....21

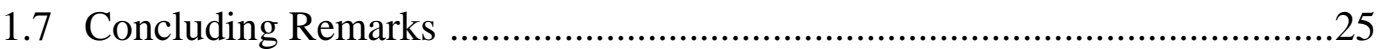

II. Host Genetics and the Gut Microbiota Additively Confer Adenoma Susceptibility in the Min Mouse Model of Colorectal Cancer .................................27

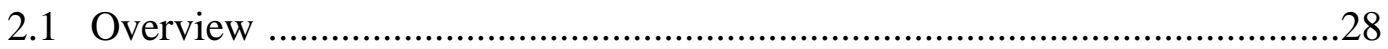

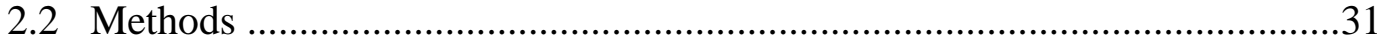

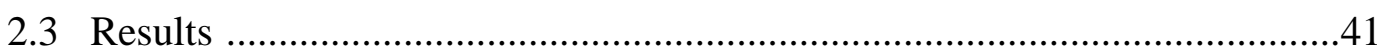

2.3.1. Genetic lineage and GM colonization additively determine adenoma susceptibility in distinct C57BL/6-Apc ${ }^{M i n}$ colonies 
2.3.2. Distinct GM communities influence adenoma susceptibility

2.3.3. GM and host genetic lineage shape the metabolome in Min mice .....44

2.3.4. Host genetic lineage influences bile acid metabolism .45

2.3.5. The Min/D Fabp6 variant associates with SI adenoma susceptibility...46

2.3.6. Colonic adenoma susceptibility is associated with changes in bile acid metabolism .47

2.4. Discussion .48

2.5. Author Contributions and Acknowledgements .55

2.6. Financial Support .55

2.7. Figures .56

2.8. Tables .73

III. Bilophila wadsworthia Supplementation Modulates the Gut Microbiota, Metabolome, and Tumor Burden in Min mice $\ldots \ldots \ldots \ldots \ldots \ldots \ldots \ldots \ldots \ldots . \ldots . \ldots . \ldots . \ldots 6$

3.1. Overview .87

3.2. Methods .89

3.3. Results .95

3.3.1. Bilophila wadsworthia modulates the gut microbiota .95

3.3.2. B. wadsworthia supplementation suppresses SI adenomas in Min ...96

3.3.3. B. wadsworthia modulates the fecal and serum metabolome .98

3.3.4. B. wadsworthia modulates fecal and serum bile acid composition .....99

3.3.5. Untargeted metabolites correlate with B. wadsworthia and adenoma multiplicity 100

3.4. Discussion .101 
3.5. Author Contributions and Acknowledgements......................................106

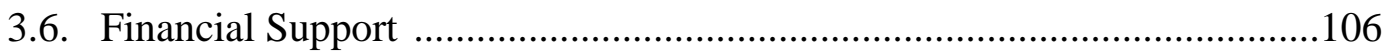

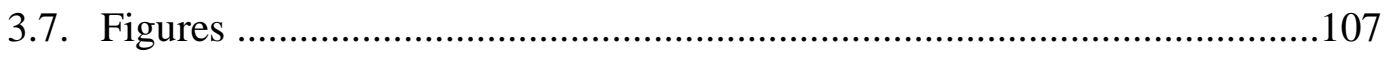

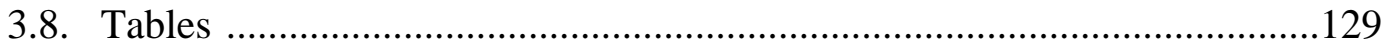

IV. The Gut Microbiota Modulates Differential Adenoma Suppression by B6/J and B6/N Genetic Backgrounds in Min Mice.....................................147

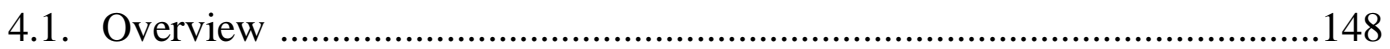

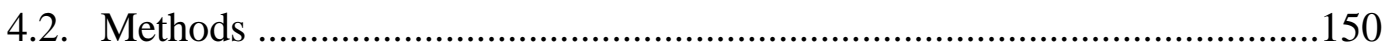

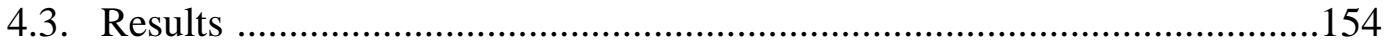

4.3.1. B6JB6JDF1-Apc ${ }^{\mathrm{Min}}$ mice exhibit partial repression of the B6/JD

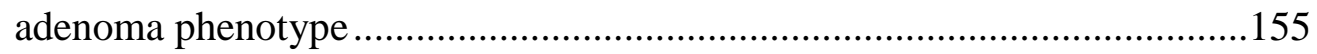

4.3.2. $\mathrm{B} 6 / \mathrm{N}$ repression of adenomas is modulated by the gut microbiota . 155

4.3.3. Distinct GMs confer differential adenoma susceptibility .................156

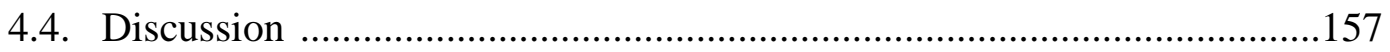

4.5. Author Contributions and Acknowledgements......................................160

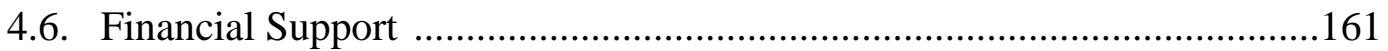

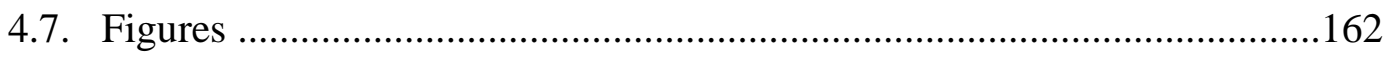

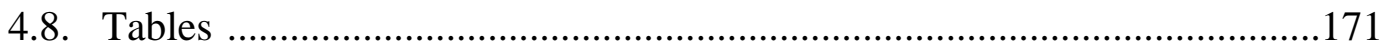

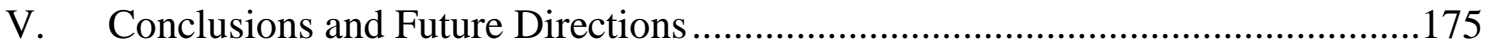

APPENDIX A: Effects of Akkermansia muciniphila Supplementation on Min Adenoma

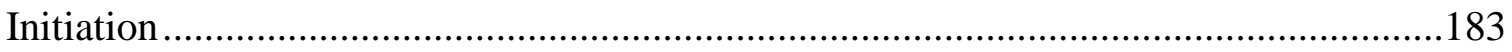

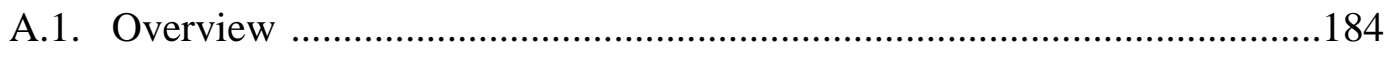

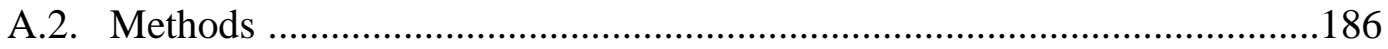


A.3.1. Akkermansia muciniphila demonstrates maternal transmission to Min offspring

A.3.2. Akkermansia. muciniphila modulates colonic goblet cells and mucin secretion.

A.3.3. Akkermansia muciniphila colonization does not influence Min adenoma multiplicity

A.4. Discussion

A.5. Financial Support

A.6. Figures

APPENDIX B: Cross fostering as a Method of Complex Microbiota Transfer .204

B.1. Overview .205

B.2. Methods .206

B.3. Results and Figures .207

B.4. Discussion .212

BIBLIOGRAPHY .214

VITA .234 


\section{LIST OF ABBREVIATIONS}

\begin{tabular}{|c|c|}
\hline $\mathrm{ACF}$ & Aberrant Crypt Foci \\
\hline ANOVA & Analysis of variance \\
\hline AOM & Azoxymethane \\
\hline$A P C$ & Adenomatous polyposis coli \\
\hline ATCC & American Type Culture Collection \\
\hline B6/J & $\mathrm{C} 57 \mathrm{BL} / 6 \mathrm{~J}$ \\
\hline $\mathrm{B} 6 / \mathrm{N}$ & C57BL/6NHsd \\
\hline BA & Bile acid \\
\hline $\mathrm{BD}$ & Becton Dickinson \\
\hline $\mathrm{CA}$ & California \\
\hline $\mathrm{CF}$ & Cross-foster \\
\hline $\mathrm{CFU}$ & Colony forming unit \\
\hline CMTR & Complex microbiota targeted rederivation \\
\hline CRC & Colorectal cancer \\
\hline DCA & Deoxycholic acid \\
\hline DNA & Deoxyribonucleic acid \\
\hline DSS & Dextran sodium sulfide \\
\hline ETBF & Enterotoxigenic Bacteroides fragilis \\
\hline ENU & $N$-ethyl- $N$-nitrosurea \\
\hline FAPB6 & Fatty acid binding protein 6 \\
\hline FAP & Familial adenomatous polyposis \\
\hline FC & Fold-change \\
\hline FDR & False discovery rate \\
\hline GC & Goblet cell \\
\hline GCMS & Gas chromatography/Mass spectrometry \\
\hline GF & Germ-free \\
\hline GI & Gastrointestinal tract \\
\hline GM & Gut microbiota/gut microbiome \\
\hline GWAS & Genome-wide association studies \\
\hline HNPCC & Hereditary nonpolyposis colorectal cancer \\
\hline HRM & High resolution melt \\
\hline IBD & Inflammatory bowel disease \\
\hline $\mathrm{IFN} \gamma$ & Interferon gamma \\
\hline IL & Interleukin \\
\hline IN & Indiana \\
\hline JAX & The Jackson Laboratory \\
\hline KEGG & Kyoto Encyclopedia of Genes and Genomes \\
\hline LCMS & Liquid chromatography/Mass spectrometry \\
\hline LDA & Linear discriminant analysis \\
\hline M & Male \\
\hline Min & Multiple Intestinal Neoplasia \\
\hline $\operatorname{Min} / \mathrm{J}$ & C57BL6/J-Apc $c^{\operatorname{Min}}$ \\
\hline $\operatorname{Min} / \mathrm{D}$ & C57BL6/JD-Apc $c^{\operatorname{Min}}$ \\
\hline MO & Missouri \\
\hline
\end{tabular}




$\begin{array}{ll}\text { Mom } & \text { Modifier of Min } \\ \text { MS/MS } & \text { Tandem mass spectrometry } \\ \text { MSI } & \text { Microsatellite instability } \\ \text { MU } & \text { University of Missouri } \\ \text { MUMC } & \text { MU Metagenomics Core } \\ \text { NA } & \text { Not applicable/available } \\ \text { NE } & \text { Normal epithelium } \\ \text { NGS } & \text { Next-generation sequencing } \\ \text { NIH } & \text { National institute of health } \\ \text { NSAID } & \text { Nonsteroidal anti-inflammatory } \\ \text { OTUs } & \text { Operational taxonomic unit(s) } \\ \text { PAST } & \text { Paleontological statistics } \\ \text { PBS } & \text { Phosphate-buffered saline } \\ \text { PCA } & \text { Principal component analysis } \\ \text { PCoA } & \text { Principal coordinate analysis } \\ \text { PCR } & \text { Polymerase chain reaction } \\ \text { PERMANOVA } & \text { Permutational multivariate analysis of variance } \\ \text { PICRUSt } & \text { Phylogenetic investigation of communities by reconstruction of } \\ & \text { unobserved states } \\ \text { PIRC } & \text { Polyposis in rat colon } \\ \text { qPCR } & \text { Quantitative polymerase chain reaction } \\ \text { qRT-PCR } & \text { Quantitative real-time polymerase chain reaction } \\ \text { RNA } & \text { Ribonucleic acid } \\ \text { ROS } & \text { Reactive oxygen species } \\ \text { SCFA } & \text { Short chain fatty acid } \\ \text { SEM } & \text { Standard error of the mean } \\ \text { SI } & \text { Small intestine } \\ \text { SMO } & \text { Spermine Oxidase } \\ \text { SNP } & \text { Single nucleotide polymorphism } \\ \text { SPF } & \text { Specific-pathogen free } \\ \text { SRB } & \text { Sulfate-reducing bacteria } \\ \text { Tau } & \text { Taurine } \\ \text { TGCA } & \text { The Cancer Genome Atlas } \\ \text { Th1 } & \text { T-helper cell type 1 } \\ \text { Th17 } & \text { T-helper cell type 17 } \\ \text { TM } & \text { Trademark } \\ \text { TN } & \text { Tennessee } \\ \text { UB } & \text { Uncultured bacterium } \\ \text { USA } & \text { United States of America } \\ \text { UW } & \text { University of Wisconsin } \\ \text { WD } & \text { Western diet } \\ \text { WGS } & \\ \text { WT } & \end{array}$




\section{LIST OF FIGURES}

\section{CHAPTER II}

Figure 1. Genetic lineage and GM colonization additively determine adenoma numbers in $A p c^{\text {Min }}$ mice. .56

Figure 2. Distinct GM communities influence adenoma susceptibility .58

Figure 3. Untargeted analysis of GM and host genetic lineage effects on the fecal metabolome .60

Figure 4. Min/D Fabp6 variant association with SI adenoma susceptibility...... 62

Figure 5. Colonic adenoma susceptibility is associated with changes in bile acid metabolism .63

Supplementary figure 1. Effects of genetic lineage and GM on adenoma susceptibility by $\operatorname{sex}$ .65

Supplementary figure 2 . Analysis of $\alpha$ - and $\beta$-diversity measures of GMJAX and GMHSD

Supplementary figure 3. GM profile and genetic lineage modulate relative abundances of specific fecal metabolites

Supplementary figure 4. Sequencing analysis of detected Fabp6 variant .71

\section{CHAPTER III}

Figure 1. Bilophila wadsworthia modulates the GM

Figure 2. B. wadsworthia supplementation suppresses SI adenomas in $A p c^{M i n}$ mice .110 
Figure 3. B. wadsworthia modulates the fecal and serum metabolome

Figure 4. B. wadsworthia regulates fecal and serum bile acid composition.

Figure 5. Untargeted metabolites correlate with adenoma multiplicity and B. wadsworthia abundance

Supplementary figure 1 . Summary of SI and colonic adenoma sizes

Supplementary figure 2. PCA visualization of untargeted small intestinal content metabolite profiles

Supplementary figure 3. Composition of detected bile acids in SI contents

Supplementary figure 4. Untargeted fecal and serum metabolites correlate with adenoma multiplicity 124

Supplementary figure 5. Untargeted metabolites correlate with $B$. wadsworthia relative abundance

\section{CHAPTER IV}

Figure 1. B6JB6JDF1-Apc ${ }^{\text {Min }}$ mice display an intermediate adenoma phenotype 162

Figure 2. The C57BL/6NHsd genetic background and the gut microbiota modulate adenoma repression in $A p c^{M i n}$ mice

Figure 3. Distinct complex gut microbiota communities contribute to differential adenoma counts 165

Supplementary figure 1. Colonic adenoma numbers in B6/J and B6/JD parental lines and B6JB6JDF1-Apc ${ }^{\text {Min }}$ offspring 169 
Supplementary figure 2. Colonic adenoma counts in rederived F1 $A p c^{M i n}$ mice

\section{APPENDIX A}

Figure 1. Maternal transmission of supplemented A. muciniphila .......................197

Figure 2. A. muciniphila supplementation effects on colonic goblet cells

Figure 3. A. muciniphila supplementation effects on adenoma multiplicity .201

Supplementary figure 1. A. muciniphila supplementation effects on SI goblet cells .....202

\section{APPENDIX B}

Figure 1. Experimental design

Figure 2. Analysis of $\alpha$-diversity measures of CF pup recipients and surrogate dam donors .208

Figure 3. Analysis of $\beta$-diversity measures of $\mathrm{CF}$ pup recipients and surrogate dam donors .210 


\section{LIST OF TABLES}

\section{CHAPTER II}

Table 1. Summary of SI adenoma number variability between C57BL6/J-Apc ${ }^{\text {Min }}$ colonies

Supplementary Table 1. Two-way PERMANOVA community analysis of fecal GM and

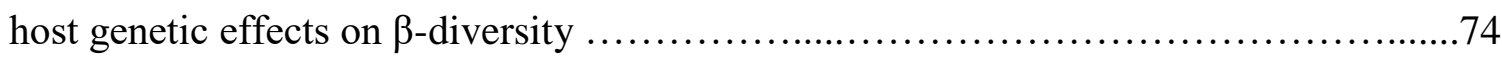

Supplementary Table 2. Two-way PERMANOVA community analysis of ileal GM and

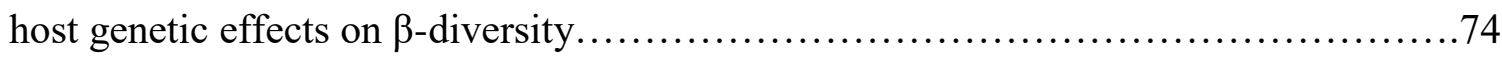

Supplementary Table 3. Significantly different fecal OTUs between GMJAX and GMHSD

Supplementary Table 4. Significantly different ileal OTUs between GMJAX and GMHSD

Supplementary Table 5. Summary of metabolic pathways modulated by genetic lineage and GM

Supplementary Table 6. Summary of heterozygous and homozygous private variants associated with $\mathrm{Min} / \mathrm{J}$ and $\mathrm{Min} / \mathrm{D}$

Supplementary Table 7. Over-represented biological pathways and candidate genes identified in $\operatorname{Min} / \mathbf{J}$

Supplementary Table 8 . Over-represented biological pathways and candidate genes identified in $\mathrm{Min} / \mathrm{D}$ 
Supplementary Table 9. RT-qPCR primer/probe sets for mouse Fabp6, Cyp39a1, Myc,

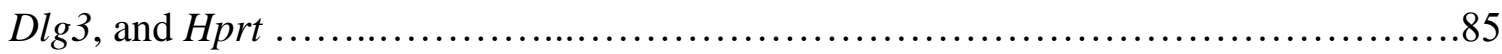

\section{CHAPTER III}

Supplementary Table 1. Significantly different OTUs in feces and ileal epithelium

following B. wadsworthia supplementation ..................................... 129

Supplementary Table 2: Summary of significantly modulated serum metabolites .......131

\section{CHAPTER IV}

Supplementary Table 1. Significantly different fecal OTUs between GM1 and GM4......171 


\begin{abstract}
Complex diseases develop as a result of genetic predisposition and environmental factors that determine individual risk. Environmental factors such as geographical location, diet, and lifestyle choices shape the gut microbiota (GM), a dynamic community of bacteria, viruses, fungi, and archaea that normally coexist symbiotically with mammalian hosts. Both genetic heterogeneity and the GM modulate various systems and influence the development of a wide range of diseases including intestinal pathologies, emphasizing the importance of the effects of genetic variants and perturbations of GM communities. Colorectal cancer (CRC) is the $2^{\text {nd }}$ leading cause of cancer-related mortality for men and women combined in the United States, and exemplifies the multifactorial etiologies that define complex diseases. In this body of work, we leveraged quantitative phenotypic variation in the $A p c$-mutant Min mouse model of CRC to interrogate genetic and microbial factors that influence adenoma susceptibility.
\end{abstract}

We used embryo rederivation to determine how genetic variation acquired through colony divergence, and distinct complex GMs representing two common mouse producers (GMJAX and GMHSD), each contribute to Min adenoma multiplicity. We found that genetic lineage and complex GM additively conferred overall adenoma multiplicity, and followed up with whole-genome sequencing (WGS) and 16S rRNA sequencing to characterize genetic divergence and GM differences, respectively. We integrated liquid chromatography coupled to mass spectrometry (LCMS) with WGS data to identify a candidate modifier variant associated with the Fabp6 gene, a regulator of bile acid reabsorption. In parallel, we identified bacteria of interest Bilophila wadsworthia and Akkermansia muciniphila associated with the adenoma-enhancing 
GMHSD, and tested the individual effects of these species on the Min phenotype. While A. muciniphila had no effect on adenoma burden, B. wadsworthia treatment resulted in modulation of the broader GM and host metabolism, and reduced adenoma multiplicity and overall burden. Finally, we asked whether the background genetics of related inbred mouse substrains C57BL/6J (B6/J) and C57BL/6NHsd (B6/N) have differential modifier effects with respect to the Min phenotype. Using F1 mice generated from B6/J or B6/N crosses to Min/D mice in combination with an embryo rederivation approach, we found that B6/N elicits stronger Min adenoma suppression than B6/J, and that the complex GM further modulates phenotype suppression. Together, this work begins to unravel the complex interactions between host and environment that drive cancer susceptibility. As such, we provide a framework for further mechanistic studies, identification of novel biomarkers, and targeted preventative and therapeutic strategies in CRC. 


\section{CHAPTER I}

An Overview of Genetic and Microbial Modifiers of Colorectal Cancer Susceptibility 


\subsection{Significance and overview of colorectal cancer}

Colorectal cancer (CRC) is the third most common cancer among men and women combined, with an estimated 145,600 new cases expected in the United States in 2019. Individual lifetime risk of developing CRC is approximately $4-5 \%$ for males and females, and is expected to cause about 51,020 deaths in 2019 (Key Statistics for Colorectal Cancer; https://www.cancer.org/cancer/colon-rectal-cancer). As such, CRC is a significant cause of morbidity and mortality warranting substantial preventative and therapeutic efforts.

$\mathrm{CRC}$ initiation and progression are characterized by hallmark changes within the intestinal epithelium. Models suggest that Aberrant Crypt Foci (ACF) are the earliest identifiable neoplastic lesions, frequently exhibiting epithelial dysplastic changes and resistance to cell death (Takayama, Katsuki et al. 1998, Alrawi, Schiff et al. 2006). Due to a persistent imbalance between cell proliferation and cell death, benign adenomas develop with continued proliferation and dysplasia. These adenomas frequently persist up to ten years, after which they may progress to carcinomas with significant potential for metastasis and mortality (de la Chapelle 2004). Observations of familial predisposition to $\mathrm{CRC}$, and the availability of biopsy material representing various stages of disease spurred decades of research focused on elucidating the underlying heritability and genetic changes that accompany the pathological progression of CRC. Early studies associated somatic mutations in the well-known oncogene ras, and loss of the tumor-suppressor p53 with various stages of disease progression (Fearon and Vogelstein 1990). While ras mutations and loss of p53 were highly associated with late-adenoma and early-carcinoma stages of CRC, these changes were notably absent from cells in early adenomas. Thus, it 
was postulated that these changes were required for progression of adenomas and malignant transformation from adenoma to carcinoma, but likely did not explain disease initiation.

Studies focused on the two most prevalent types of heritable CRC, hereditary nonpolyposis colorectal cancer (HPNCC) and Familial Adenomatous Polyposis (FAP), were critical to our understanding of adenoma initiation. HPNCC or Lynch syndrome is defined by transmission of germ-line mutations in DNA mismatch repair genes including MSH2 and $M L H 1$, resulting in destabilization of the genome referred to as microsatellite instability (MSI) (Cunningham, Christensen et al. 1998). MSI stemming from these inherited mutations not only predisposes patients to CRC, but also to many other types of cancer (Cortes-Ciriano, Lee et al. 2017). FAP, characterized by the development of hundreds to thousands of colorectal adenomas in patients, was observed as early as the mid- $18^{\text {th }}$ century and was recognized as an inherited syndrome by the start of the $20^{\text {th }}$ century. In contrast to HPNCC, tumors from FAP patients typically do not demonstrate MSI characterized by DNA mismatch repair mutations, but rather are considered microsatellite stable (MSS) (Kinzler and vogelstein 1996). Despite awareness of the hereditary nature of FAP, its underlying molecular pathogenesis was unknown until the early 1990s, when mutant alleles of the tumor-suppressor adenomatous polyposis coli (APC) demonstrated cosegregation in affected kindreds (Groden, Thliveris et al. 1991, Nishisho, Nakamura et al. 1991). Additional studies showed that in FAP, transmission of a single truncating $A P C$ mutant allele was not sufficient to induce adenoma initiation. Rather, loss of the additional wild-type $A P C$ copy via allelic loss or somatic mutation frequently precedes adenoma formation, supporting Knudson's “two-hit” hypothesis in 
which an additional mutation beyond germ-line transmission is necessary to initiate cancer (Knudson 1993).

While hereditary forms of CRC comprise approximately $20-25 \%$ of all cases, sporadic CRC where no familial predisposition is apparent, accounts for most remaining cases (de la Chapelle 2004). Notably, the critical role of $A P C$ mutation as a rate-limiting event in tumorigenesis is not limited to FAP, as somatic $A P C$ mutations are present in a majority of sporadic colorectal tumors (Miyoshi, Nagase et al. 1992, Rowan, Lamlum et al. 1999). As loss-of-function APC mutations initiate tumors in both FAP patients and sporadic CRC, $A P C$ was designated the "gatekeeper" of colonic epithelial cell proliferation, whereby its inactivation results in an imbalance of cell division and cell death (Kinzler and vogelstein 1996). Since $A P C$ has been implicated as a significant regulator of cancer initiation, its molecular role in Wnt-signaling has been wellcharacterized. Cytoplasmic APC forms part of a multi-protein complex that destabilizes $\beta$-catenin by marking it for ubiquitination and proteasome-mediated degradation. With the loss of functional APC, $\beta$-catenin accumulates in the ctyoplasm and translocates into the nucleus, where it binds $\mathrm{T}$ cell factor (TCF) and lymphoid enhancer-binding factor (LEF) and serves as a coactivator to stimulate transcription of Wnt target genes that regulate cell proliferation and death (Bienz and Clevers 2000).

More recently, human studies using CRC cohorts have linked various genetic factors with increased CRC risk through correlative studies, promoting a polygenic model of CRC susceptibility. Genome-wide association studies (GWAS) have been particularly useful in identifying new genetic susceptibility loci, suggesting important roles for loci that may affect known cancer pathways, as well as those that modify risk through other 
unknown mechanisms (Houlston, Cheadle et al. 2010, Peters, Jiao et al. 2013, Al-Tassan, Whiffin et al. 2015). Studies have also taken advantage of newer high-throughput approaches such as transcriptomic analysis to determine how differential expression of genes associate with CRC cohorts, and thereby developed genetic signatures that predict multiple cancer types (Peng, Bian et al. 2015). Taken together, these studies provide a platform for mechanistic studies that address the underlying associations uncovered by high-throughput approaches, and offer a framework to distinguish mutations in modifier genes from passenger mutations with no functional consequences.

In addition to efforts centered on genetic factors in CRC, the prevalence of sporadic disease prompted significant interest in non-heritable factors that affect CRC risk, with special focus on environmental variables that may contribute to adenoma initiation and progression. Sporadic CRC has been associated with the westernization of society, with factors including geographical location, diet, and tobacco and alcohol consumption affecting disease risk (Danaei, Vander Hoorn et al. 2005). Studies initially focused on these risk factors in the context of their capacity to induce somatic mutations that may initiate adenomas or allow adenomas to progress to cancer. For example, diets high in red meats associated with developed countries were classically linked to increased genotoxicity and overall CRC risk (Rieger, Parlesak et al. 1999, Louis, Hold et al. 2014). More recently, studies have uncovered a number of potential mechanistic links between the environment and CRC. These include increased prevalence of immune-mediated conditions such as inflammatory bowel disease (IBD) that may precede CRC, generalized or local epigenetic changes affecting expression of oncogenes or tumor-suppressors, and perturbations of the commensal microbial community residing within the GI tract 
(Cunningham, Christensen et al. 1998, Frigola, Song et al. 2006, Rubin, Shaker et al. 2012, Weir, Manter et al. 2013). Taken together, epidemiological studies of sporadic CRC emphasize the multifactorial nature of disease initiation and progression and emphasize the challenges we face in resolving the contributions of these complex variables.

\subsection{Rodent models of APC-driven CRC}

While retrospective human studies suggest a number of correlations between various genetic and environmental factors and CRC risk, these approaches are limited by their associative nature. Preclinical rodent models have revolutionized our ability to address the underlying mechanisms driving these associations, providing a platform for prospective studies investigating the molecular underpinnings of proposed correlations in large-scale studies. Due to the well-established role of $A P C$ as a tumor initiator in $80-$ $90 \%$ of CRCs, the majority of mouse models developed to recapitulate human CRC have centered on $A p c$ mutations. Of these $A p c$-focused models, the multiple intestinal neoplasia (Min; $A p c^{\mathrm{Min} /+}$ ) is the longest-standing and one of the most commonly cited cancer models (Kwong and Dove 2009, Zeineldin and Neufeld 2013). The Min mouse was originally created by $N$-ethyl- $N$-nitrosourea (ENU) mutagenesis of C57BL/6J (B6) inbred mice resulting in large numbers of small intestinal (SI) adenomas, and relatively fewer colonic adenomas (Moser, Pitot et al. 1990). Soon thereafter, the Min phenotype in ENU-mutagenized mice was mapped to an autosomal dominant nonsense mutation in the murine $A p c$ gene, leading to downstream dysregulation of Wnt-signaling ( $\mathrm{Su}$, Kinzler et al. 1992, Morin, Sparks et al. 1997). 
Since the inception and large-scale production of Min mice, studies have exploited the model's quantitative phenotype to provide a wealth of knowledge pertaining to the study of $A p c$ tumor-suppressor function, genetic modifiers, environmental variables, and chemopreventative strategies in CRC (Kwong and Dove 2009, Jackstadt and Sansom 2016). Oshima et al. classically demonstrated that for adenomas to form in Min mice, a second mutational Apc "hit" in addition to the germline mutation was required. This finding highlighted the role of loss-of-heterozygosity (LOH) mediated by homologous somatic recombination in cancer initiation (Oshima, Oshima et al. 1995). Interestingly, other studies later showed that LOH is not always required for adenoma formation in murine $A p c$ mutant models, and suggested that $A p c$ haploinsufficiency and epigenetic silencing of the WT copy can similarly lead to adenoma formation (Amos-Landgraf, Irving et al. 2012). Chemopreventative studies in Min mice demonstrated marked preventative effects of non-steroidal anti-inflammatory drugs (NSAIDs) via cyclooxygenase-2 (COX2) inhibition. These findings have translated to the use of NSAIDs as an effective prevention strategy for human FAP patients (Jacoby, Seibert et al. 2000, Baron, Cole et al. 2003). Moreover, studies incorporating Min mice were pivotal in showing how environmental factors, especially the diet, can influence tumorigenesis. Mai et al. demonstrated that calorie-restricted Min mice have reduced adenoma frequency (Mai, Colbert et al. 2003), while others have shown that a high-fat diet enhances tumor numbers (Park, Kim et al. 2016). Taken together, these studies speak to the importance of environmental and lifestyle variables, and may partially explain increased CRC prevalence in western societies. 
The plethora of data originating from the Min mouse highlights its utility as a model of early carcinogenesis. However, it is also important to recognize the limitations of this particular model as it pertains to the full scope of CRC initiation and progression. One of the most substantial challenges in the development of mouse models of CRC is generating mice that display progression to adenocarcinomas with metastatic disease (Jackstadt and Sansom 2016). CRC progression is frequently characterized by compounding mutations in genes such as KRAS, TP53, PTEN, and PI3KCA that promote disease progression following APC-driven initiation (Jackstadt and Sansom 2016). However, these changes are not observed in Min mice, and thus the phenotype rarely progresses. To improve the model's resemblance to human disease, Apc mutants have been combined with Kras, Pik3ca, and Pten mutants to generate mice with more invasive adenocarcinomas (Sansom, Meniel et al. 2006, Marsh, Winton et al. 2008).

Min mice also display a primarily SI phenotype, unlike human FAP where most tumors arise in the colon. The combination of $A p c$ mutation with mutations in the transcription factor encoded by $C d x 2$ results in a considerable shift towards a colonic phenotype, though this also seems to increase chromosomal instability (Aoki, Tamai et al. 2003). Amos-Landgraf et al. circumvented this problem by creating the Polyposis in rat colon (Pirc) rat model, which like the Min mouse harbors a knockout allele in the Apc gene, but exhibits a colonic tumor distribution that recapitulates human FAP (AmosLandgraf, Kwong et al. 2007). Furthermore, the larger size of the rat more readily permits longitudinal analysis and sampling through endoscopic techniques, and there is no evidence of increased chromosomal instability. Altogether, an acute awareness of the strengths and limitations of the Min mouse is required for its optimal usage as a model of 
CRC. Under appropriate experimental conditions, the Min quantitative phenotype serves as a powerful platform for the interrogation of genetic and environmental contributions to CRC, and potential chemopreventative and therapeutic strategies.

\subsection{Phenotypic variability in Min}

When the Min mouse was originally created and maintained on the B6 inbred background at the University of Wisconsin, mice developed an average of approximately 100 SI adenomas (Amos-Landgraf, Heijmans et al. 2014). However, investigators soon found that the Min allele conferred highly variable overall tumor susceptibility depending upon genetic background (Kwong and Dove 2009). Notable examples include the BTBR background, which confers an enhanced phenotype of approximately 600 tumors (Kwong, Shedlovsky et al. 2007), and the AKR background, which suppresses the phenotype to just a few tumors per animal (Shoemaker, Moser et al. 1998). The powerful effects of background genetics on Min prompted efforts to uncover potentially translational novel modifying loci, defined as loci that would otherwise have no phenotypic effect in the absence of the Apc mutation. A well-known modifier is the Mom1 (Modifier of Min 1) locus, which was identified by quantitative trait loci (QTL) analysis using a series of crosses between inbred strains (Dietrich, Lander et al. 1993). Further resolution of this locus confirmed that the Pla2g2a gene which encodes secretory phospholipase 2A, has resistance and sensitivity alleles that account for significant phenotypic variability between background strains (Cormier, Bilger et al. 2000). Subsequently, similar mapping strategies were used to elucidate additional modifier loci including Mom2, Mom3, Mom7, Mom12, and Mom13 (Kwong and Dove 2009). Mom3 
and Mom7 were mapped to regions of chromosome 18 proximal to Apc, suggesting that they could have a role in the rate of somatic recombination and LOH (Kwong, Shedlovsky et al. 2007). However, this chromosomal region also contains the complex centromeric sequence, which complicates efforts to resolve these loci by sequencing.

Additional studies have recognized modifiers that influence tumorigenesis by induction of mitotic instability, hyper-recombination, and both enhancing and suppressing effects of epigenetic machinery (Goss, Risinger et al. 2002, Millar, Guy et al. 2002, Sansom, Berger et al. 2003, Rao, Yang et al. 2005). For example, the BubRl and Blm genes both have important roles in maintaining genomic stability. When loss-offunction mutations in these genes are combined with $A p c$ mutation in Min mice, reduced genomic stability and subsequently higher rates of somatic recombination result in dramatically increased adenoma multiplicity (Goss, Risinger et al. 2002, Rao, Yang et al. 2005). $M b d 2$, whose function is required to recruit co-suppressor complexes to methylated DNA, is required for adenoma formation in Min mice (Sansom, Berger et al. 2003). This suggests that epigenetic silencing at the Apc locus, in addition to other loci, contributes to adenoma initiation. More recently, investigators have adopted alternative strategies to identify modifiers of Min using a systems genetics approach. Dorman et al. crossed Min mice with a panel of 27 different high-diversity collaborative cross (CC) lines to generate F1 offspring. Interestingly, the F1 offspring displayed a broad range of tumor multiplicities depending on the $\mathrm{CC}$ line. These results suggest that several dominant modifiers are present in the CC lines (Dorman, Baer et al. 2016). However, high numbers of genetic modifiers will likely complicate resolution efforts, and further 
studies are required to determine whether novel modifiers translate to human CRC susceptibility.

Interestingly, Min phenotypic variability is not limited to the presence of genetic modifiers across different inbred genetic backgrounds, as variability is also readily apparent between different Min colonies on the B6 genetic background. Phenotypic variability within the B6 background became especially apparent after a sub-population of the original UW Min colony (Min/D) was sent to The Jackson Laboratory (JAX) for production and global distribution. C57BL/6J-Apc ${ }^{M i n} / \mathrm{J}$ mice from JAX $(M i n / \mathrm{J})$ exhibited average SI tumor multiplicity ranging from approximately 20 total tumors in some colonies to 130 in others (MacGregor, Kim et al. 2000, Kwong, Shedlovsky et al. 2007, Zell, Ignatenko et al. 2007). Presently, it is unknown whether these phenotypic shifts are attributable to institutional environmental differences or genetic drift. However, this observation emphasizes the potential for genetic modifiers of Min to appear spontaneously within a colony despite maintenance on the same genetic background. The Mom2 locus, which mapped to a distal region of chromosome 18 , is an example of such a modifier. This locus was further resolved to show that a duplication in the ATP synthase Atp5al gene confers substantial resistance to tumorigenesis via cell lethality, and could therefore explain tumor suppression in some of the Min colonies originating from JAX (Baran, Silverman et al. 2007). Presently, it is unclear what drives the appearance of these functional variants in some colonies versus others. Though rarely considered, colony maintenance practices may influence the rate of phenotypically consequential genetic drift in specific Min colonies. For example, the original Min/D colony was consistently progeny tested to ensure that only male breeder lines with high adenoma multiplicities 
were used, causing the colony to maintain consistently high adenoma numbers. In contrast, large-scale production facilities typically allow males to breed for the duration of their reproductive lifespan regardless of phenotype. This practice inherently facilitates higher contributions to the overall population from healthier males that on average have fewer adenomas Therefore, we may select for variants that confer fewer adenomas over the course of several generations in large production colonies where male breeders are not screened for adenoma multiplicity.

Phenotypic variability within a relatively uniform genetic background also suggests that environmental factors that may contribute to Min phenotype disparity across colonies. Colnot et al. designed an alternative Apc mutant that shifted the adenoma phenotype to the colon and rectum (Colnot, Niwa-Kawakita et al. 2004). The authors noted that the colorectal phenotype was more severe in conventionally housed mice compared to those in pathogen-free conditions, indicating the importance of unknown environmental influences between and even within institutions. Differences in personnel, husbandry standards, water decontamination methods, and pathogen exclusion among other practices between mouse producers and institutions can all impact the resident intestinal flora, or gut microbiota (GM) (Bidot, Ericsson et al. 2018, Montonye, Ericsson et al. 2018). To that end, Ericsson et al. demonstrated that mice with the same inbred genetic background from the common mouse producers JAX and Envigo have highly distinct GMs in terms of both specific taxa present, and overall richness and diversity of the intestinal communities (Ericsson, Davis et al. 2015). Though it is not yet clear how these communities affect Min, investigators using other models of intestinal disease have already leveraged this natural variation to determine potential GM-mediated modifying 
effects on a phenotype of interest. In a study focused on IBD, Hart et al. demonstrated that different complex GMs confer variable disease severity in the genetically-susceptible IL-10 ${ }^{-/-}$mouse model (Hart, Ericsson et al. 2017). Thus, naturally occurring differential GMs resulting from environmental differences between mouse producers or other institutions may contribute to phenotypic variability across colonies that share the same genetic background.

\subsection{The Microbiome in CRC}

Trillions of bacteria that reside within the GI tract comprise the complex and dynamic system known as the gut microbiota (GM). The vast majority of these bacteria are classified into highly abundant phyla such as Bacteroidetes and Firmicutes, but also include less common phyla such as Proteobacteria (Mariat, Firmesse et al. 2009). These communities are highly prevalent in the colon relative to the small intestines, where they range from low abundances in the duodenum to higher abundances in the terminal ileum (Sekirov, Russell et al. 2010). Recent focus on the role of the GM in maintaining homeostatic conditions highlights a number of critical functions of these intestinal communities including mucosal immune development, host metabolic homeostasis, drug metabolism, and gut barrier integrity (Jandhyala, Talukdar et al. 2015). With the identification of the GM as a significant regulator of both local and systemic physiology, perturbation of the normal intestinal flora is increasingly recognized as a potentially pathogenic event (Round and Mazmanian 2009, Sekirov, Russell et al. 2010). Several studies have attributed cancer initiation to the intestinal environment through evaluation of fecal water genotoxicity, and found that increased toxicity associated with various risk 
factors could contribute to carcinogenesis (Hebels, Sveje et al. 2012, Daniela, Sara et al. 2014). While the source of this genotoxicity is unclear, these observations suggest that microbial communities and their metabolic products that comprise the intestinal environment likely contribute. The GM's relationship with CRC is of particular interest due to disease localization within the GI tract, a known relationship between the GM and predisposing inflammatory conditions, and CRC's complex multifactorial etiology.

Early studies investigating the GM primarily depended on cultivating bacterial communities with identification based on growth in selective media, biochemical characteristics, and morphology and staining properties. Though these approaches were relatively successful, they were limited by an inability to culture the majority of commensal species found in the GI tract. Contemporary high-throughput approaches enable culture-independent GM characterization through sequencing technology, and therefore circumvent labor-intensive bacterial cultivation and identification. Among the most common approaches for identification and relative quantification of GM taxa is the use of next-generation sequencing (NGS) of the microbial 16S rRNA gene in DNA extracted from fecal samples or other samples throughout the GI tract. The 16S rRNA gene encodes a component of the prokaryotic ribosome that is highly conserved between bacterial species, and therefore serves as a PCR target to generate amplicons for sequencing. Hypervariable regions within the 16S rRNA gene further enable phylogenetic classification of sequenced amplicons into operational taxonomic units (OTUs) by comparison to previously annotated sequences in established databases (Weisburg, Barns et al. 1991, Coenye and Vandamme 2003, Ritari, Salojarvi et al. 2015). Alternatively, shotgun sequencing of the whole metagenome provides a comprehensive 
survey of the GM, and further enables functional annotation of microbial communities (Tringe, von Mering et al. 2005). Together, these approaches provide improved understanding of GM communities, and allow characterization of abundance, richness, and diversity measures in the context of health and disease.

Following the availability of culture-independent methods of GM analysis, several studies used human CRC cohorts to draw associations between enriched or depleted microbial communities and disease status, intending to identify potentially pathogenic or protective flora. Enrichment of individual taxa such as enterotoxigenic Bacteroides fragilis, pathogenic E. coli strains, and Akkermansia muciniphila among others is associated with CRC populations, while butyrate-producing bacteria such as Roseburia and others within family Lachnospiraceae are reportedly more prevalent in healthy cohorts (Wang, Cai et al. 2012, Weir, Manter et al. 2013, Fukugaiti, Ignacio et al. 2015). Though these correlative studies are valuable as hypothesis-generating observations, there are significant challenges when interpreting results. Namely, distinction of true pathologic agents from bystanders that benefit from the tumor microenvironment is limited by the retrospective nature of most clinical studies. Furthermore, lack of reproducibility across clinical studies highlights our inability to design prospective longitudinal studies and limitations in controlling genetic and environmental variation in human cohorts. For example, some studies suggest enrichment of Prevotella spp. in healthy patients (Weir, Manter et al. 2013), while others report its enrichment in the mucosa and stool associated with CRC (Sobhani, Tap et al. 2011, Chen, Liu et al. 2012). Similarly, sulfate- and sulfite-reducing bacteria (SRB) of the family Desulfovibrionaceae, which produce large amounts of hydrogen sulfide $\left(\mathrm{H}_{2} \mathrm{~S}\right)$ 
have a disputed role in CRC. While some cite enrichment of SRBs and increased genotoxic $\mathrm{H}_{2} \mathrm{~S}$ in CRC (Attene-Ramos, Wagner et al. 2006, Yazici, Wolf et al. 2017), others have reported no differences in relative abundance of SRB communities, or suggest a protective anti-inflammatory function (Balamurugan, Rajendiran et al. 2008, Scanlan, Shanahan et al. 2009). Taken together, these challenges emphasize the importance of employing translatable animal models to systematically address observations made in associative studies.

Studies in various preclinical models of CRC have assessed the role of the GM at both a community level and a mechanistic level with respect to specific taxa. For such studies, the ability to reduce variation by using standardized complex GMs, simplified communities, and the availability of germ-free (GF) mice devoid of any detectable intestinal microbial flora are distinct advantages to using animal models. Li et al. observed that GF Min mice developed significantly fewer adenomas compared to their conventionally colonized counterparts, indicating an important role for the GM in adenoma initiation (Li, Kundu et al. 2012). Zackular et al. showed that antibioticmediated depletion of the GM in azoxymethane/dextran sodium sulfate (AOM/DSS) treated mice resulted in significantly fewer intestinal tumors compared to mice that did not receive antibiotics, and thus corroborated the importance of the GM. To follow, GF mice colonized with a GM from tumor-bearing mice resulted in a higher tumor burden compared to those colonized with a GM from healthy mice, suggesting that tumorassociated communities are capable of exacerbating disease (Zackular, Baxter et al. 2013). 
Associative clinical studies and community-based GM studies in animal models have spurred interest in a number of bacterial species and thus prompted investigators to directly interrogate their role in CRC. Fusobacterium spp. are commonly enriched in colorectal carcinomas relative to surrounding normal tissue, and have therefore garnered attention as potentially pathogenic species (Chen, Liu et al. 2012, Kostic, Gevers et al. 2012). To address these associations, Kostic et al. supplemented Min mice with Fusbacterium nucleatum daily for 8 weeks, and subsequently observed a proinflammatory profile and potentiated colonic tumorigenesis (Kostic, Chun et al. 2013). Meanwhile, a robust study incorporating fecal samples from over 500 patients representing a range of CRC stages found that there was no enrichment of Fusobacterium at the adenoma stage despite its association with advanced carcinomas (Amitay, Werner et al. 2017). Coupled with the fact that colonization and tumor potentiation in Min mice required daily Fusobacterium nucleatum treatments for a two month period, Fusobacterium's role as an oncogenic driver versus bystander remains contentious.

Wu et al. also utilized the Min model as a platform to explore the oncogenic role Enterotoxigenic Bacteroides fragilis (ETBF), an organism that secretes $B$. fragilis toxin (BFT) and can cause inflammatory diarrhea in people. Notably, chronic colonization of Min mice with ETBF triggers colitis and promotes colonic tumorigenesis via T helper type 17 (Th17)-mediated activation of signal transducer and activator of transcription-3 (Stat3) (Wu, Rhee et al. 2009). Additional studies in Min show that inhibition of IL-17A independent of ETBF treatment, suppresses adenomas, and thus supports a critical role for Th17 immune responses in Min adenomagenesis (Chae, Gibson et al. 2010). Moreover, ETBF-mediated inflammation induces the polyamine enzyme spermine 
oxidase (SMO), whose activity results in increased reactive oxygen species (ROS) and DNA damage. A blockade of SMO catabolism reduced ETBF-mediated colon tumorigenesis, demonstrating that microbial induced enzymes can directly influence tumor initiation (Goodwin, Destefano Shields et al. 2011). While ETBF is a primary example of a bacterial-induced Th17 response, other species known to trigger Th17 have uncharacterized roles in oncogenesis. For example, Prevotella spp. are part of the normal oral and intestinal flora, but enrichment of this genera is associated with augmented Th17-mediated mucosal inflammation (Larsen 2017). Based on previous work centered on ETBF, further studies exploring tumorigenic roles for these species are warranted.

\subsection{Microbial metabolites and CRC}

Much of our understanding of the GM's role in CRC susceptibility and pathogenesis stems from diet-microbe interactions, and the metabolic products of these interactions. Enterohepatic bile acid (BA) metabolism is a primary example of the interdependence of diet, host physiology, and the GM. Primary bile acids are synthesized from cholesterol in the liver, and in mice, are conjugated exclusively with taurine, and either taurine or glycine in humans. Bile acids contain both methyl groups and hydroxyl groups, and are therefore amphipathic. This property enables BAs to emulsify lipid aggregates, and facilitates solubilization and transport of lipids in aqueous environments. Thus, BAs are critical for the digestion and absorption of lipids within the small intestines. (Chiang 2009). The majority of BAs are reabsorbed through the SI epithelium via transporters such as apical sodium-dependent bile salt transporter (ASBT) and fatty acid-binding protein 6 (FABP6/IBABP) as they pass through the terminal ileum (Trauner 
and Boyer 2003). However, 5-10\% of BAs are not reabsorbed, and instead undergo multiple bacterial biotransformations in the ileum, cecum, and colon. In this process, primary BAs are first deconjugated by microbes with bile salt hydrolase (BSH) activity, followed by $7 \alpha$-dehydroxylation to generate secondary BAs such as deoxycholic acid (DCA) and lithocholic acid (LCA) (Zeng, Umar et al. 2019). BSH bacteria are primarily gram-positive species from the genera Clostridium, Bifidobacterium, and Lactobacillus, whereas $7 \alpha$-dehydroxylating activity is largely limited to clostridial species such as Clostridium scindens (Wells, Williams et al. 2003, Ridlon, Kang et al. 2006, Ridlon, Harris et al. 2016). It is also important to note that BAs can alter influence the GM. For example, the release of cholic acid following consumption of a high-fat source results in an increase in the Firmicutes:Bacteroidetes ratio (Islam, Fukiya et al. 2011). Moreover, the presence of taurine in conjugated bile acids provides a source of sulfite that promotes expansion of the sulfite-reducing bacteria Bilophila wadsworthia, leading to the production of $\mathrm{H}_{2} \mathrm{~S}$ via sulfite reduction (Peck, Denger et al. 2019).

An extensive body of literature implicates secondary BAs as a risk factor for early carcinogenesis. Elevated levels of unconjugated fecal BAs have long been associated with human CRC or polyp cohorts, prompting a number of studies exploring their carcinogenic potential (Imray, Radley et al. 1992, Ajouz, Mukherji et al. 2014). Cao et al. treated Min mice with DCA and observed both increased adenoma multiplicity and acceleration of the adenoma-adenocarcinoma sequence, suggesting DCA's role in both tumor initiation and progression. These DCA-mediated changes were associated with an increased percentage of $\beta$-catenin positive cells (Cao, Luo et al. 2014). Later studies showed that even low concentrations of DCA increase phosphorylation of $\beta$-catenin and 
induce downstream targets urokinase-type plasminogen activator (uPAR) and cyclin D1, and thus confirmed its carcinogenic activity (Pai, Tarnawski et al. 2004). Other proposed mechanisms of secondary BA oncogenicity include targeting of nuclear receptor signaling (Degirolamo, Modica et al. 2011), perturbation of epithelial cell membranes due to increased hydrophobicity (Ridlon, Wolf et al. 2016), and increased epidermal growth factor receptor-mediated COX-2 expression (Cheng and Raufman 2005). Studies investigating factors that regulate microbial communities that produce secondary BAs are warranted due to the extensive evidence suggesting the carcinogenicity of these metabolites.

In addition to intestinal bacteria that regulate BA metabolism, host genetic factors interacting with dietary components are also of critical importance. Western diets (WD) high in fat result in BA expansion in the intestines to facilitate lipid digestion, and enhance cell proliferation and tumorigenesis in B6 mice. Moreover, WD triggers dysregulation of BA metabolism through reduced expression of the BA transporters FABP6 and ASBT (Dermadi, Valo et al. 2017), disrupting the normal enterohepatic axis and potentially increasing luminal BAs available for biotransformation. Thus, genetic variants that alter expression of genes encoding BA transporters are of interest as potential modifiers of BA-mediated carcinogenesis.

In addition to high fat intake, scarce fiber consumption typifies a WD associated with increased CRC risk, while vegetarian diets high in fiber are linked to decreased risk (Dermadi, Valo et al. 2017). Accumulating epidemiological and molecular evidence suggest that GM-mediated metabolism of fiber has an important role in CRC susceptibility. The GM utilizes various polysacchararides, oligosaccharides, and lignin 
that comprise dietary fiber as a source to produce short chain fatty acids (SCFA) including butyrate, acetate, and propionate (Zeng, Umar et al. 2019). This processing is mutually beneficial for the host and microbiota, as bacterial metabolism of complex sugars provides a microbial energy source while breaking down otherwise indigestible dietary components for host utilization. Butyrate is produced by Coprococcus spp., Anaerostipes spp., Eubacterium spp., Roseburia spp., and Faecalibacterium prausnitzii among others, and has been studied extensively with respect to its effects on cellular homeostasis, colonic cell proliferation, and CRC prevention (Barcenilla, Pryde et al. 2000, Scott, Martin et al. 2006). Butyrate undergoes $\beta$-oxidation within the mitochondria of colonic epithelial cells, accounting for a majority of energy used by normal colonocytes (Roediger 1980). Butyrate is also a strong histone deacetylase (HDAC) inhibitor and promotes cycle arrest and apoptosis in CRC cell lines through Wnt signaling hyperactivation (Bordonaro and Lazarova 2015). Finally, a high fiber diet protected gnotobiotic AOM/DSS treated mice in a microbiota- and butyrate-dependent manner, such that only those mice colonized with the butyrate-producing Butyrivibrio fibrisolvens demonstrated fiber-induced tumor suppression (Donohoe, Holley et al. 2014). To summarize, BA and SCFA metabolism are primary examples of how the GM modulates host physiology, and thereby affect overall CRC susceptibility.

\subsection{From characterization to function: tools to assess the host-GM interactions}

The development of tools that enable interrogation of GM function in the context of both human cohorts and disease models is critical as interest in the GM's role in various pathophysiological processes continues to grow. Bioinformatics tools frequently 
do not account for dynamic community interactions, and functional annotation of $16 \mathrm{~S}$ rRNA sequencing data is lacking (Zierer, Jackson et al. 2018). Ideally, novel tools will account not only for the function of complex microbial communities, but also molecular interactions between host and GM that can provide mechanistic insight into GMmediated disease modulation.

New techniques allow for initial assessment of whether the complex GM modulates a phenotype of interest, serving as a platform for further functional studies. Hart et al. developed Complex Microbiota Targeted Rederivation (CMTR), a strategy that leverages natural variation in complex GM between common mouse producers (Hart, Ericsson et al. 2018). In CMTR, embryos are isolated from a colony with a genetic background including any mutations of interest, and rederived into surrogate dams harboring different complex GMs. As a result, isogenic animals representing the genetics of interest are colonized with distinct complex GMs through maternal transmission, permitting the evaluation of how each GM contributes to the phenotype of interest. Following the development of CMTR, the approach was initially used to demonstrate that complex GM modulates disease severity in the IL- $10^{-/-}$mouse model of IBD (Hart, Ericsson et al. 2017). To follow, Ericsson et al. demonstrated the utility of CMTR in a model of CRC by rederiving isogenic Pirc rats using surrogate dams harboring three distinct GMs (Ericsson, Akter et al. 2015). Analysis of the tumor phenotype revealed that one of the complex GMs conferred a significantly reduced tumor burden. Notably, one of the rats colonized with the suppressive GM failed to develop any colonic tumors, indicating that this GM community disrupted the full penetrance of the Pirc allele. Thus, the IL-10 $0^{-/}$and Pirc models provide a foundation for the utility of CMTR when 
determining the role of the complex GM in a given phenotype. While this approach serves as a baseline to determine how the GM influences disease models, follow-up techniques are required to assess how the GM functionally modulates these pathologies.

High-throughput transcriptomic and metabolomics profiling are rapidly advancing our understanding of host-microbe interactions and their relationships to normal physiology and disease. Several studies have employed RNA-sequencing of host tissue to fundamentally show how changes in commensal communities alter host gene expression. For example, circadian rhythm dictates oscillations in localization and abundance of several mucosa-associated GM taxa, which is accompanied by coinciding oscillations in the host transcriptome (Thaiss, Levy et al. 2016). Antibiotic mediated disruption of the GM also abrogated these cyclic transcriptomic changes, confirming that molecular changes associated with circadian fluctuation are partially mediated by the GM through a number of described pathways (Thaiss, Levy et al. 2016). Meanwhile, others have taken more targeted approaches towards understanding how specific commensals alter host gene expression. Adult volunteers were supplemented with the commensal Lactobacillus plantarum, then subjected to duodenal biopsy for transcriptional profiling. The authors found that $L$. plantarum regulated expression of a number of host genes including upregulation of Wnt targets MYC and cyclin D1, suggesting the commensal's role in modulating cell proliferation within the SI (van Baarlen, Troost et al. 2009). Pfalzer et al. employed an integrated GM, metabolomics, and transcriptomics analysis in a model of obesity-induced intestinal cancer. Regression analysis demonstrated correlations between several tumor-associated taxa and cancer-related gene expression, especially those that regulate the PI3k-Akt pathway (Pfalzer, Kamanu et al. 2016). Though these results do not 
prove causation and fail to show which changes precede tumor formation, they provide the framework for such studies.

Untargeted metabolomics allows evaluation of the global metabolic state of a given environment. Metabolite separation techniques such as liquid or gas chromatography are frequently coupled with mass spectrometry (LCMS, GCMS) to generate small molecule profiles from complex mixtures (DeHaven, Evans et al. 2010). As such, metabolomics may be considered a terminal view of a biological system that accounts for underlying genetic factors, transcriptional products, and all enzymatic or protein-mediated reactions (Spratlin, Serkova et al. 2009). Thus, metabolomics can also account for environmental factors, including the GM, that contribute to metabolic shifts that may be relevant to disease pathogenesis (Wikoff, Anfora et al. 2009, Zierer, Jackson et al. 2018). Gnotobiotics and antibiotic-mediated microbiota depletion have been used extensively to show that the GM contributes to host metabolite profiles. For example, Theriot et al. used antibiotics to assess GM-mediated metabolite profile shifts in mice susceptible to Clostridium difficile infection. Of note, the authors found that GM depletion resulted in enrichment of primary BAs and several carbon sources, which $C$. difficile subsequently used to enhance its own colonization and thus exacerbate disease (Theriot, Koenigsknecht et al. 2014).

An improved understanding of metabolites in relation to associated microbes and CRC is critical to further development of metabolite and prebiotic-based preventatives and therapeutics. Non-digestible oligosaccharides and fiber are considered useful supplements in the prevention of CRC (Fotiadis, Stoidis et al. 2008), and in vitro data demonstrates the potential clinical use of rice bran extracts for their chemopreventative 
properties (Forster, Raina et al. 2013). Moreover, studies have shown that metabolites found in milk fermented by specific Lactobacillus and Streptococcus spp. display antimutagenic effects that may translate to reduced cancer risk (Wollowski, Rechkemmer et al. 2001). These advances suggest that metabolomics-based approaches have significant potential to elucidate unknown metabolites that can promote beneficial microbes, or directly influence CRC pathogenesis.

With respect to CRC, several epidemiological studies have utilized metabolomics to identify differential metabolite profiles between healthy and disease cohorts (Holmes, Li et al. 2011, Weir, Manter et al. 2013, Sinha, Ahn et al. 2016). These efforts have identified CRC-associated metabolites and pathways that are subject to GM modulation, including BA and sphingolipid metabolism, SCFA production, and CRC-specific amino acid composition among many others. While it is unclear whether these metabolic shifts occur prior to, or after disease initiation and progression, these studies provide a platform for further mechanistic exploration. Given the complexity of the genome, the GM, and their composite metabolic product, sophisticated approaches incorporating model systems are required to resolve contributing variables. Based on these challenges, prospective studies that incorporate microbiota manipulation techniques and high-throughput approaches in the context of $\mathrm{CRC}$ models will better inform targeted preventative and therapeutic strategies.

\subsection{Concluding Remarks}

Epidemiological studies have paved the way for a deeper understanding of CRC susceptibility largely through identification of foundational genetic and environmental 
risk associations. While these associations are limited in their capacity to inform underlying causative factors in $\mathrm{CRC}$, animal models overcome many of these limitations by enabling prospective longitudinal studies that target the role of both individual and complex variables. The Min model of early intestinal neoplasia emerged as a quantitative platform to test whether a wide range of variables modify disease susceptibility. However, meta-analysis of the Min phenotype reveals dramatic phenotypic variability of unknown origin across different colonies, which presents significant challenges with respect to reproducibility and translatability of studies using this model. Though extensive studies indicate that both genetic modifiers and environmental variables influencing GM community structure can alter the Min phenotype, our understanding of these host-microbe interactions and how they confer overall CRC susceptibility is significantly lacking. Therefore, studies that utilize novel approaches and techniques to address how complex factors contribute to overall risk and pathogenesis are critical to the advancement of personalized prevention and the development of new treatment strategies. 


\section{CHAPTER II}

\section{Host Genetics and the Gut Microbiota Additively Confer Adenoma Susceptibility in the Min Mouse Model of Colorectal Cancer}

(Jacob Moskowitz, Anthony Doran, Zhentian Lei, Susheel Busi, Marcia Hart, Craig

Franklin, Lloyd W. Sumner, Thomas Keane, and James Amos-Landgraf) 


\subsection{Overview}

Colorectal cancer $(\mathrm{CRC})$ is a complex disease trait resulting from a variety of factors including genetic predisposition, diet, age, inflammation, and lifestyle (Thune and Lund 1996, de la Chapelle 2004, Itzkowitz and Yio 2004). Malignant disease is preceded by the initiation of adenomas in the epithelial lining of the intestinal mucosa, and often persist up to ten years before acquiring malignant transformations, making the adenoma a critical target for early intervention (Bertagnolli, Hsu et al. 2010). Recently, CRC has been associated with perturbations in the gut microbiota (GM) through postulated mechanisms including modulation of inflammation, genotoxin production, and metabolic homeostasis (Travaglione, Fabbri et al. 2008, Cuevas-Ramos, Petit et al. 2010, Carbonero, Benefiel et al. 2012, Couturier-Maillard, Secher et al. 2013), but it is often unclear whether these shifts in bacterial composition directly impact disease risk, or merely result from physiological changes associated with disease. Initiation and progression of adenomas is likely determined by a combination of genetic factors and changes in microbial populations that mutually impact relevant pathways (Birt and Phillips 2014). However, the ability to successfully integrate these complex factors and to dissect the independent and additive effects of each remains elusive in human populations.

The intestinal environment is collectively comprised of dynamic interactions between diet, modified host compounds, and microbial metabolites (Franzosa, SirotaMadi et al. 2019). As such, changes in host functional genetic output via germline or acquired mutations, or shifts in the functional GM, may substantially influence the metabolite profile. Metabolomics provides an avenue to interrogate the metabolic output 
of complex biological systems in a non-targeted discovery-based approach (Bino, Hall et al. 2004). Metabolomics represent a highly sensitive means of detecting functional changes associated with genetic variation, differences in complex microbial communities, and even more importantly the combination of these factors in the context of complex disease traits. Several studies have already demonstrated the utility of metabolomics in colorectal cancer, identifying microbial metabolites including short-chain fatty acids (SCFAs) such as butyrate that can influence gene expression, cell proliferation, and ultimately adenoma formation (Bultman and Jobin 2014). Furthermore, altered levels of microbial-influenced metabolites including bile acids (BA) and hydrogen sulfide $\left(\mathrm{H}_{2} \mathrm{~S}\right)$ are associated with both inflammatory bowel disease and CRC through the production of genotoxic reactive oxygen species (Attene-Ramos, Wagner et al. 2007, Carbonero, Benefiel et al. 2012, Dossa, Escobar et al. 2016, Farhana, Nangia-Makker et al. 2016). Thus, non-targeted metabolomics has been used extensively to identify novel metabolites associated with CRC (Melnik, da Silva et al. 2017).

Adequate models are needed to study initiating events in CRC due to the challenges of controlling environmental conditions and performing longitudinal monitoring of disease progression from pre-disease stages in human populations. The $A p c^{\text {Min }}$ (Min) mouse model of CRC, which harbors an autosomal dominant mutation in the Apc tumor suppressor gene causing the development of intestinal adenomas, provides an extensively studied platform to interrogate genomic and GM contributions to disease initiation in a quantitative manner (Shoemaker, Gould et al. 1997). Investigators using this model have observed complex genetic modification of the adenoma phenotype from multiple modifier genes, including modifiers between mouse strains and newly arising 
variants within the C57BL/6J strain (Cormier, Bilger et al. 2000, Baran, Silverman et al. 2007, Kwong and Dove 2009). It is now clear that in addition to both known and unknown genetic factors, the GM can also impact adenoma initiation and progression, as germ-free Min mice develop significantly lower adenoma burdens than their colonized counterparts (Li, Kundu et al. 2012). Still, it is unclear how functional genomic changes and distinct GM communities independently and additively influence adenoma initiation in the context of the complex specific-pathogen-free GM. Thus, the Min mouse provides a platform to dissect genomic and microbial contribution to phenotypic variability and to draw further inferences about variable disease susceptibility across human populations.

Adenoma counts across different colonies have varied substantially over the past two decades of use of the C57BL/6J-Apc ${ }^{+/ M i n}$ mouse, (Table 1). In some cases, these disparities were attributed to undetermined differences between institutions. It is wellestablished that mice originating from different mouse producers and institutions have highly distinct GMs (Ericsson, Davis et al. 2015). Furthermore, strict genetic control of mouse models is essential to maintaining a consistent phenotype. Though producers take precautions to prevent genetic drift in inbred colonies, mutations in genetic modifiers of the Min phenotype may appear rapidly within a colony, and thus account for differences in tumor number across different colonies. In this study, we used the observed phenotypic variability between two Min mouse colonies from a common lineage that have been separated in excess of twenty generations, to interrogate whether disparity in tumor numbers between C57BL/6 inbred colonies occurs due to differences in the GM or host genetic differences associated with colony divergence. We transferred embryos from mice from a low-tumor multiplicity colony (C57BL6/J-Apc ${ }^{M i n}$ abbrv. Min/J) and a high- 
tumor multiplicity colony (C57BL6/JD-Apc $c^{\text {Min }}$ abbrv. Min/D) into surrogate dams harboring distinct complex GMs, resulting in two independent lines of mice each harboring two distinct complex GMs. We describe independent and additive influences of host genetics and the GM on adenoma initiation through the use of 16S rRNA microbial profiling, host whole-genome sequencing (WGS), and finally non-targeted metabolomics. This approach allowed for the relatively non-invasive identification of altered biologically relevant pathways and mechanistic associations with CRC initiation through integration and refinement of large data sets.

\subsection{Methods}

\subsubsection{Animal use and ethics statement}

Animal studies were conducted in an Association for Assessment and Accreditation of Laboratory Animal Care International (AAALAC) accredited facility according to the guidelines provided by the Guide for the Care and Use of Laboratory Animals, and were approved by the University of Missouri Institutional Animal Care and Use Committee. For Complex Microbiota Targeted Rederivation (CMTR), C57BL/6JMlcr-Ap$c^{M i n} / \mathrm{Mmmh}(M i n / \mathrm{D})$ and C57BL/6J-Apc ${ }^{M i n} / \mathrm{J}(M i n / \mathrm{J})$ embryos were transferred into separate $\mathrm{Crl}: \mathrm{CD} 1$ surrogate dams with distinct complex GM populations (GMJAX and GMHSD) to naturally deliver offspring representing four experimental groups; Min/J $\mathrm{JMJAX}_{\mathrm{G}} \operatorname{Min} / \mathrm{D}_{\mathrm{GMJAX}}, \operatorname{Min} / \mathrm{J}_{\mathrm{GMHSD}}$, and $\operatorname{Min} / \mathrm{D}_{\mathrm{GMHSD}}$ (Fig. 1A).

Male and female CMTR offspring were group-housed by sex, genetic origin of the embryo donor $(\mathrm{Min} / \mathrm{D}$ or $\mathrm{Min} / \mathrm{J})$, and GM of the surrogate dam (GMJAX or GMHSD). All mice, including embryo donors, ET recipients, and rederived offspring 
were group-housed in microisolator cages on ventilated racks (Thoren, Hazelton, PA) on a 14:10 light:dark cycle on paper chip bedding (Shepherd Specialty Papers, Watertown, TN), with ad libitum access to 5058 irradiated breeder chow (LabDiet, St. Louis, MO) and acidified autoclaved water. All pups were ear-punched at weaning (21 days of age) using sterile technique. DNA was extracted using the "HotSHOT" genomic DNA preparation method as described (Truett, Heeger et al. 2000). We generated N2 backcross animals by crossing $\mathrm{Min} / \mathrm{D}$ males and WT females from the $\mathrm{Min} / \mathrm{J}$ colony to first create F1 hybrids of the two genetic lineages. F1 hybrids were then backcrossed to both the $\mathrm{Min} / \mathrm{D}$ and $\mathrm{Min} / \mathrm{J}$ parental lines to create N2 mice. At 3 months of age, all mice were euthanized via $\mathrm{CO}_{2}$ asphyxiation and the abdominal cavity was opened. Whole small and large intestines were incised longitudinally, flushed with saline and placed on bibulous paper with the luminal side facing up for formalin fixation. Grossly visible adenomas were counted manually using a Leica M165FC microscope at $1.25 \mathrm{x}$ magnification. Fecal samples were collected from all rederived mice at 1 month, while fecal samples, cecal material, and ileal scrapes were collected after sacrifice at 3 months of age. For onemonth fecal samples, individual mice were placed into an empty autoclaved cage, and fecal pellets were collected immediately following natural bowel movements.

\subsubsection{Genotyping}

Genotyping for the Min allele by PCR was performed in a reaction volume of 10 uL containing $0.2 \mathrm{uM}$ of each primer (5'-ATTGCCCAGCTCTTCTTCCT-3' and 5'CGTCCTGGGAGGTATGAATG-3'), 1 x HRM Supermix (BioRad, Hercules, CA), and genomic DNA. Genotyping for the Fabp6 upstream deletion was similarly performed using ear-punches as described. The $10 \mathrm{uL}$ HRM reaction contained $0.2 \mathrm{uM}$ of each 
primer (5'-ACCACTTCCTCCCTCAGGAT-3' and 5'-TTCTCCCAATGCCCATCCAG3'), 1 x HRM Supermix, and genomic DNA. Genotyping reactions and analyses were carried out using a BioRad CFX384 Real-Time PCR Detection system. For Min genotyping, cycling conditions were as follows: $95^{\circ} \mathrm{C}, 2 \mathrm{~min} ; 40$ cycles of $95^{\circ} \mathrm{C}, 10 \mathrm{sec}$; $60^{\circ} \mathrm{C}, 30 \mathrm{sec}, 72^{\circ} \mathrm{C}, 30 \mathrm{sec}, 95^{\circ} \mathrm{C}, 30 \mathrm{sec} ; 60^{\circ} \mathrm{C}, 1 \mathrm{~min}$, followed by melt curve analysis from $73^{\circ} \mathrm{C}$ to $85^{\circ} \mathrm{C}$ in increments of $0.1^{\circ} \mathrm{C}$ for $10 \mathrm{sec}$. PCR cycling conditions for Fabp6 analysis were the same as those mentioned above, followed by a melt curve analysis from $65^{\circ} \mathrm{C}$ to $95^{\circ} \mathrm{C}$ in increments of $0.2^{\circ} \mathrm{C}$. All melt curve results were analyzed using BioRad Precision Melt Software v1.2 to detect the Min allele or the Fabp6 deletion.

\subsubsection{Embryo collection and transfer}

Embryos for transfer were collected from donors from two separate colonies (ET donors). Half of the embryos were obtained from frozen stocks that were generated through breeding of sexually mature C57BL/6JD-Apc ${ }^{+/ M i n}(\mathrm{Min} / \mathrm{D})$ males with 5-8 weekold C57BL/6JD-Apc ${ }^{+/+}$females, maintained as a closed-colony at the McArdle Laboratory, University of Wisconsin (Madison, WI). A second cohort of embryos for ET was obtained on-site (University of Missouri, Columbia, MO) using C57BL/6J-Apc ${ }^{+/ M i n}$ $(\mathrm{Min} / \mathrm{J})$ males and five to eight week-old C57BL/6J- $\mathrm{Apc}^{+/+}$females, purchased from The Jackson Laboratory (Bar Harbor, ME). To generate Min/J embryos, in vitro fertilization was performed by the University of Missouri Cryobiology Core as described (Takeo T 2011). Presumptive zygotes were then moved to a KSOM dish and incubated for 24 hours to allow progression to the two-cell stage (Biggers JD 2000). For ET recipients, eight week old CD1 females harboring a GM (Hsd:CD1 ${ }_{\mathrm{GMHSD}}$ ) from Envigo (Envigo, Indianapolis, IN) were purchased and allowed to acclimate for one week prior to use. 
Eight week old CD1 females harboring a GM representing The Jackson Laboratory

$\left(\mathrm{Crl}: \mathrm{CD} 1_{\mathrm{GMJAX}}\right)$ were previously generated in our laboratory (Hart, Ericsson et al. 2018). $\mathrm{CD} 1_{\mathrm{GMHSD}}$ and $\mathrm{CD} 1_{\mathrm{GMJAX}}$ surrogate female embryo recipients were mated with sterile, vasectomized Hsd:CD1 or Crl:CD1 males, respectively. All surrogate females were inspected for copulatory plugs and plug-positive mice were used for embryo transfer. Surrogate females were anesthetized via IM injection of ketamine/xylazine cocktail at 5.5 $\mathrm{mg}$ and $1 \mathrm{mg}$ per $100 \mathrm{~g}$ body weight respectively, and placed in sternal recumbency. A dorsal midline incision was made and the uterine oviducts located by dissecting through the retroperitoneal muscle. Embryos in 3 to $5 \mu \mathrm{l}$ of media were injected into the oviducts using a glass hand-pipette. Skin incisions were closed with sterile surgical staples and mice received a subcutaneous injection of $2.5 \mathrm{mg} / \mathrm{kg}$ of body weight flunixin meglumine (Banamine®) prior to recovery on a warming pad.

\subsubsection{Tissue collection and processing}

All mice were humanely euthanized with $\mathrm{CO}_{2}$ asphyxiation and necropsied, and small intestines were processed as described above. A sterile scalpel blade was used to gently scrape normal ileal and colonic epithelium. After the body cavity was opened, whole spleens and liver were also collected. All collected tissue was flash-frozen in liquid nitrogen followed by storage at $-80{ }^{\circ} \mathrm{C}$.

\subsubsection{Sample collection and DNA extraction for 16S rRNA sequencing}

Two fecal pellets per mouse were collected aseptically and placed in a $2 \mathrm{~mL}$ round-bottom tube containing $800 \mu \mathrm{l}$ of lysis buffer (Ericsson, Davis et al. 2015) and a $0.5 \mathrm{~cm}$ diameter stainless steel bead (Grainger, Lake Forest, Il). All samples were mechanically disrupted using a TissueLyser II (Qiagen, Venlo, Netherlands) for 2 
minutes at $50 \mathrm{~Hz}$, followed by incubation at $70^{\circ} \mathrm{C}$ for 20 minutes with periodic vortexing. DNA extraction from fecal pellets, cecal contents, and ileal epithelium for 16S rRNA sequencing was performed using a DNeasy Blood \& Tissue Kit@ (Qiagen) as previously described (Ericsson, Davis et al. 2015).

\subsubsection{S library preparation and sequencing}

Using DNA extracted as described in Section 2.2.5, bacterial 16S rRNA amplicons were generated by amplification of the V4 hypervariable region of the $16 \mathrm{~S}$ rRNA gene using universal primers (U515F/806R) (Caporaso, Lauber et al. 2010), then sequenced on the Illumina MiSeq platform as described previously (Ericsson, Davis et al. 2015). Assembly, binning, and annotation of DNA sequences were performed using Qiime v1.9 (Kuczynski, Stombaugh et al. 2012) at the University of Missouri Informatics Research Core Facility (Columbia, MO) as described (Ericsson, Davis et al. 2015). Contiguous sequences were assigned to operational taxonomic units (OTUs) using a criterion of $97 \%$ nucleotide identity by de novo clustering. Taxonomy was assigned to selected OTUs using BLAST (Altschul, Madden et al. 1997) against the SILVA database (Ritari, Salojarvi et al. 2015) of 16s rRNA sequences and taxonomy.

\subsubsection{Tissue processing and Reverse Transcriptase-quantitative PCR (RT-qPCR)}

Ileal scrapes collected at 3 months of age were used to quantitate expression of Fabp6, Myc, and Dlg3, and liver used to quantitate expression of Cyp39al. All collected tissues were flash-frozen in liquid nitrogen followed by storage at $-80{ }^{\circ} \mathrm{C}$. Frozen tissues were mechanically disrupted using a TissueLyser II (Qiagen) for 4 minutes at $50 \mathrm{~Hz}$. Total RNA was then extracted using the AllPrep ${ }^{\circledR}$ DNA/RNA Mini Kit (Qiagen), and cDNA was synthesized using the iScript ${ }^{\mathrm{TM}}$ cDNA Synthesis Kit (Bio-Rad, Hercules, CA) 
according to the respective manufacturer's instructions. Samples were analyzed in quadruplicate and all evaluated gene expression levels were normalized to Hprt expression using a PrimeTime ${ }^{\circledR}$ qPCR assay $\left(\right.$ IDT $^{\circledR}$ ) and the $2^{-\Delta \Delta C t}$ method of relative expression (Schmittgen and Livak 2008). For qPCR, each $10 \mathrm{uL}$ reaction contained $1 \mathrm{x}$ Primer/Probe mixes (Table S9), 1 x iTaq $^{\mathrm{TM}}$ Universal Probe Supermix, and 100 ng cDNA template. PCR parameters were: denaturation at $95^{\circ} \mathrm{C}$ for 5 seconds, and annealing and elongation at $60^{\circ} \mathrm{C}$ for 30 seconds for a total of 54 cycles.

\subsubsection{Whole-Genome sequencing}

Genomic DNA for whole-genome sequencing (WGS) was extracted from splenic tissue using the DNeasy Blood \& Tissue Kit®, as described by the manufacturers (Qiagen). Paired-end (151 base pair) sequence reads generated for each sample were aligned to the GRCm38 (mm10) mouse reference genome using BWA-MEM (v0.7.5) (http://arxiv.org/abs/1303.3997) followed by a local realignment around indels using the GATKv3.0 ‘IndelRealigner Tool’ (McKenna, Hanna et al. 2010). Possible PCR and optical duplicates were filtered using Picard tools (v1.64)

(http://broadinstitute.github.org/picard). SNP and short indel calls were generated using the Mouse Genomes Project variation catalog v5 parameters (Doran, Wong et al. 2016). In brief, samtools mpileup v1.3 (Li, Handsaker et al. 2009) and vcftools call v1.3 (Danecek, Auton et al. 2011) were used to identify SNPs and indels in each of the samples. Indels were left-aligned using the bcftools norm function. Filters were then applied to remove variants of low depth ( $<10$ reads), low genotype quality $(q<20)$, poor mapping quality $(\mathrm{q}<20)$ and proximity to an indel (SNPs within $2 \mathrm{bp}$ of an indel). Additionally, only heterozygous SNPs with > 5 support reads for each allele were 
retained. Functional consequences based on mouse Ensembl gene models (v88) were annotated using the Variant Effect Predictor (McLaren, Pritchard et al. 2010). The VEP tool facilitates the identification of synonymous and deleterious mutations such as stop changes and potentially damaging missense variants. Variants private to each sample were identified by removing SNPs and indels common to any of the 36 strains present in the MGPv5 catalog (Doran, Wong et al. 2016).

\subsubsection{TA cloning and Sanger sequencing for variant validation}

Ear punches were used to collect DNA for variant validation as described in Genotyping Section 2.2.2. To validate the observed variant in the upstream region of Fabp6 detected by WGS, this region was PCR amplified using the primers FWD 5'ACCACTTCCTCCCTCAGGAT-3', REV 5'-TTCTCCCAATGCCCATCCAG-3'. The TOPO TA Cloning ${ }^{\circledR}$ Kit (Invitrogen ${ }^{\mathrm{TM}}$ ) was used to insert the region of interest into the $\mathrm{pCR}^{\mathrm{TM}} 4-\mathrm{TOPO} \circledast$ vector, and TOP10 competent $E$. coli cells were used for vector transformation according to the manufacturer's instructions. Transformed cells were spread onto Lysogeny Broth (LB) plates with $50 \mathrm{ug} / \mathrm{mL}$ kanamycin for resistance selection, then grown overnight at $37^{\circ} \mathrm{C}$ in a shaking incubator. The PureYield ${ }^{\mathrm{TM}}$ Plasmid Miniprep System (Promega, Madison, WI) was used to extract DNA from each culture according to the manufacturer's instructions. Sequencing reactions were prepared using the extracted DNA and the T7 sequencing primer (5'-TAATACGACTCACTATAGGG3'. Sanger sequencing was performed at the MU DNA Core using a 3730xl 96-capillary DNA analyzer (ThermoFisher Scientific, Waltham, MA) with the Applied Biosystems Big Dye Terminator cycle sequencing chemistry. 


\subsubsection{Ultra-high performance liquid chromatography-tandem mass spectrometry (UHPLC-MS/MS)}

Fecal samples weighing $25 \mathrm{mg}$ were extracted with $1.0 \mathrm{~mL} 80 \% \mathrm{MeOH}$ containing $18 \mu \mathrm{g} / \mathrm{mL}$ umbelliferone as an internal standard, sonicated for 5 minutes and centrifuged for 40 minutes at $3000 \mathrm{~g}$ at $10^{\circ} \mathrm{C} .0 .5 \mathrm{~mL}$ supernatant was used for UHPLCMS analysis after centrifugation at $5000 \mathrm{~g}$ at $10^{\circ} \mathrm{C}$ for 20 minutes and $250 \mu \mathrm{L}$ of the extract transferred into autosampler glass vials with $300 \mu \mathrm{L}$ inserts. A Bruker maXis impact quadrupole-time-of-flight mass spectrometer coupled to a Waters ACQUITY UPLC system was used to perform UHPLC-MS analyses. Metabolite separations were achieved using a Waters BEH C18 column (2.1x $150 \mathrm{~mm}$ with 1.7-um particles) using a linear gradient composed of mobile phase A ( $0.1 \%$ formic acid) and B (100\% acetonitrile). Phase B increased from 5\% to $70 \%$ over $30 \mathrm{~min}$, then to $95 \%$ over $3 \mathrm{~min}$, held at $95 \%$ for $3 \mathrm{~min}$, then returned to 5\% for equilibrium. The flow rate was 0.56 $\mathrm{mL} / \mathrm{min}$ and the column temperature was $60{ }^{\circ} \mathrm{C}$. Mass spectrometry was performed in the negative electrospray ionization mode with the nebulization gas pressure at $43.5 \mathrm{psi}$, dry gas of $12 \mathrm{l} / \mathrm{min}$, dry temperature of $250^{\circ} \mathrm{C}$ and a capillary voltage of $4000 \mathrm{~V}$. Mass spectral data were collected from 100 and $1500 \mathrm{~m} / \mathrm{z}$ and were auto-calibrated using sodium formate after data acquisition. One sample from each of the four experimental groups was analyzed with automated MS/MS. Fragmentation data was compared to archived PUBCHEM and KEGG fragment databases via the MetFrag web tool (https://msbi.ipb-halle.de/MetFragBeta/).

\subsubsection{Metabolomics data analysis}


Chromatographic data were aligned using mass and retention time with XCMS software (http://xcmsonline.scripps.edu/). Following alignment, XCMS was used to generate a relative intensity table with individual features labeled by retention time and mass for analysis using the Metaboanalyst v3.0 web program (Xia, Sinelnikov et al. 2015). In Metaboanalyst, the interquartile range method was used to filter data. Data were normalized based on sample sums of features' relative intensity, then log transformed prior to multivariate analysis. Principle Component Analysis (PCA), putative metabolite identification, and pathway overrepresentation cloud plots were generated with XCMS, where dysregulated pathways were determined using the mummichog algorithm $(\mathrm{Li}$, Park et al. 2013). Metaboanalyst was used to perform hierarchical clustering using the Euclidean distance measure and Ward clustering algorithm with significantly modulated (based on ANOVA) metabolites according to experimental group, and displayed as a heat-map and dendogram. Metabolite and tumor correlation analyses were performed using small intestinal tumor counts and relative intensities for individual features across all four experimental groups, and regression graphs were generated using GraphPad Prism 8. Individually significant features were determined separately in terms of GM (compared Min/D $\mathrm{D}_{\mathrm{GMJAX}}$ and $\mathrm{Min} / \mathrm{D}_{\mathrm{GMHSD}}$ ) and genetic lineage (compared of $\operatorname{Min} / \mathrm{J}_{\mathrm{GMJAX}}$ and Min/ $\mathrm{D}_{\mathrm{GMJAX}}$ ) by t-test in XCMS. To determine the metabolites contributing to the separation and rooting of the hierarchical clusters illustrated by the dendogram, the samples were classified into those with 'high' or 'low' colonic adenoma numbers independent of genetic lineage or GM, and a linear discriminant analysis (LDA) was performed using the LEfSe (Linear discriminant analysis Effect Size) tool on a highcomputing Linux platform (Segata, Izard et al. 2011). An LDA score of $\log 10=2$ or 
greater for any given metabolite was considered significantly differential between the high and low adenoma groups.

\subsubsection{Statistical analyses}

Statistical analyses were performed using Sigma Plot 14.0 (Systat Software Inc., Carlsbad CA). Differences in OTU relative abundance between GMJAX and GMHSD were determined using Student's t-test. To account for multiple testing, OTUs with a $p$ value $<0.001$ were considered statistically significant. Two-way ANOVA with the Student Newman-Keuls post-hoc method was used to assess differences in adenoma number between rederived groups, where $p<0.05$ was considered statistically significant. For GM analysis, GraphPad Prism 8 was used to generate bar graphs and Tukey's box plots displaying phylum relative abundances, richness (OTU counts), and $\alpha$ diversity (Shannon Index). Principal Coordinate Analyses incorporating the Bray-Curtis similarity index used for visualizing $\beta$-diversity were generated with the Paleontological Statistics software package (PAST) 3.12 (Hammer, Harper et al. 2001). Two-way ANOVA/Student Newman-Keuls post-hoc method was used to assess differences in richness and $\alpha$-diversity and phylum abundance differences between rederived mice. To better account for quantitative and qualitative community differences between GMJAX and GMHSD, statistical testing for $\beta$-diversity was performed via a two-way PERMANOVA analysis of both Bray-Curtis and Jaccard dissimilarities for bacterial OTU community structure using PAST 3.12. For RT-qPCR assays, expression analysis was performed using the $2^{-\Delta \Delta \mathrm{Ct}}$ method of relative expression (Schmittgen and Livak 2008), and statistical differences were assessed using the Student's t-test. 


\subsection{Results}

\subsubsection{Genetic lineage and GM colonization additively determine adenoma susceptibility in distinct $\mathrm{C57BL/6-Apc^{Min }}$ colonies}

Historically, tumor multiplicities in C57BL/6- $A p c^{\operatorname{Min}}$ mice vary widely in reported studies despite having the same inbred genetic background (Table 1). Notably, these colonies were housed in different institutions for unknown numbers of generations prior to reporting tumor numbers, highlighting the difficulty in separating the impact of genetic divergence from environmental variables. We compared intestinal adenoma number in our institution between two C57BL/6- $A p c^{M i n}$ lines arising from a common colony. The original B6-Apc ${ }^{\text {Min }}$ colony was developed in the McArdle Laboratory at the University of Wisconsin $(M i n / \mathrm{D})$. A subset of Min/D mice were sent to the Jackson Laboratory (JAX) and underwent rederivation for colony development and distribution $(\mathrm{Min} / \mathrm{J})$, and thus harbor a GM representing JAX. The original Min/D colony was maintained as a closed colony through sibling mating and harbored a GM from Harlan/Sprague Dawley (now Envigo) that was acquired through pup fostering to CD-1 (Harlan ID) foster mice to rid the colony of Helicobacter spp. Mice from the Min/D colony had an average of 99.2 small intestinal (SI) and 2.26 colonic adenomas (Amos-Landgraf JM 2014), and breeder males were consistently progeny-tested to maintain tumor multiplicities in the offspring within the average. The Min/J colony acquired from the Jackson Laboratory and maintained at the University of Missouri developed 44.2 and 0.55 SI and colonic adenomas, respectively. Thus, the Min/J colony develops fewer adenomas than the Min/D colony throughout the GI tract $(p<0.001)$ (Fig. 1A). 
We used Complex Microbiota Targeted Rederivation (CMTR) to interrogate how GM and host genetic lineage independently and additively contribute to variable adenoma susceptibility in $A p c^{M i n}$ mice. We established mice from the $\mathrm{Min} / \mathrm{J}$ and $\mathrm{Min} / \mathrm{D}$ genetic lineage with two different complex GMs; a low-richness GM originally acquired from B6 mice from the Jackson Laboratory (GMJAX) and high-richness GM originally acquired from CD-1 mice from Envigo (GMHSD). These GM profiles were chosen because they most closely represent the original GMs of the $\mathrm{Min} / \mathrm{J}$ and $\mathrm{Min} / \mathrm{D}$ colonies, respectively. Min/J and Min/D embryos were separately implanted into surrogate dams harboring the desired GM, such that they would maintain their original genetic lineage while acquiring the desired maternal GM through natural birth. Thus, we generated four experimental groups representing each combination of genetic lineage and GM (Fig. 1B). All $A p c^{M i n}$ offspring were sacrificed at 3 months of age, and SI and colonic adenomas were counted to determine the effects of genetic lineage and GM colonization on adenoma susceptibility. We found that mice colonized with GMHSD developed more SI adenomas than their GMJAX counterparts independent of genetic lineage. Furthermore, mice of the $\mathrm{Min} / \mathrm{D}$ lineage developed more adenomas than $\mathrm{Min} / \mathrm{J}$ mice independent of GM when comparing adenoma susceptibility between the genetic lineages within each GM (Fig. 1C). Thus, colonization of Min/J embryos with GMHSD partially restored the original Min/D phenotype, but did not account entirely for the phenotypic differences between the original Min/D and Min/J colonies. Colonization of Min/D embryos with GMJAX suppressed the original Min/D phenotype, while colonization of Min/D with GMHSD completely restored the original McArdle phenotype. Min/ $\mathrm{D}_{\mathrm{GMHSD}}$ mice develop substantially more adenomas than $\operatorname{Min} / \mathrm{J}_{\mathrm{GMJAX}}(p<0.001)$ when the effects of genetic 
lineage and GM are combined. In the colon, we observed increased adenomas in GMHSD-colonized mice compared to GMJAX, while genetic lineage had no apparent effect (Fig. 1C). These trends were similarly observed when males and females were assessed separately (Fig. S1). To summarize, both genetic lineage and GM colonization independently modulated adenoma susceptibility, and collectively had either additive protective or deleterious phenotypic effects.

\subsubsection{Distinct GM communities influence adenoma susceptibility}

Feces were collected at 1 month, and fecal and ileal epithelial scrapes were collected at 3 months of age from rederived $A p c^{M i n}$ mice to characterize the GMJAX and GMHSD microbial communities. 16S rRNA sequencing was used to determine the relative abundance of all detected microbial taxa. At 1 month, phyla Proteobacteria, Actinobacteria, Deferribacteres, and Cyanobacteria were enriched in GMHSD-colonized mice, while Tenericutes were enriched in GMJAX-colonized mice (Fig. 2A). These changes were observed regardless of genetic lineage, indicating that phylum make-up was determined by the surrogate dam rather than genetic lineage of the embryo. At the operational taxonomic unit (OTU) level, GMJAX and GMHSD had distinct post-weaning microbial profiles in fecal samples (Fig. 2B) which remained disparate until sacrifice at 3 months in both feces and ileal scrapes (Fig. S2A). Community analyses of fecal and ileal $\beta$-diversity by two-way PERMANOVA corroborated the discrete nature of these communities (Table S1 and S2). Sex did not appear to play a significant role in GM make-up, and as anticipated based on previous characterization of these GMs (Ericsson, Davis et al. 2015), GMHSD mice had increased microbial richness (Chao1 index) and $\alpha$ diversity (Shannon Index) compared to GMJAX mice (Figs. S2B and S2C). We found 58 
and 34 significantly modulated OTUs in feces and ileal scrapes, respectively, between GMJAX and GMHSD, using a $p$-value of 0.001 as a threshold (Tables S3 and S4). GMHSD mice harbored enriched abundances of sulfidogenic Desulfovibrio and Bilophila sp., as well as sulfatase-secreting bacteria (SSB) Rikenella, while GMJAX had enriched levels of Bacteroides sp. and family Peptococcaceae. A heat map illustrating fold-change of the 25 most significantly modulated OTUs was used for a hierarchical clustering analysis, and shows that samples clustered based on GM profile, regardless of genetic lineage (Fig. 2C). Thus, GMJAX and GMHSD represent highly distinct complex microbial communities with a number of different taxa potentially contributing to differential adenoma susceptibility.

\subsubsection{GM and host genetic lineage shape the metabolome in $A p c^{M i n}$ mice}

Based on the results of our rederivation experiment, we aimed to determine functional differences between each genetic lineage and GM community that could contribute to differential disease susceptibility using a metabolomics approach. Feces contains not only microbial metabolites, but also mammalian host metabolites that may undergo microbial biotransformation (Franzosa, Sirota-Madi et al. 2019). In untargeted analyses of fecal metabolites at 3 months of age detected by ultra-high pressure liquid chromatography coupled mass spectrometry (UHPLC-MS), we observed distinct metabolite profiles based on both genetic lineage and GM colonization (Fig. 3A). Using a False Discovery Rate (q-value) of 0.1 as a threshold, we found that 1009 features were significantly modulated between the four rederived $A p c^{\text {Min }}$ groups. Of these features, 172 were specifically modulated by the GM and 7 by genetic lineage (Supplementary datasets 1-3; Figs. S3A and B), while the remainder appear to be modulated by a combination of 
the two factors. A heat map illustrating fold-change of the most substantially modulated metabolites (based on ANOVA) was used for a hierarchical clustering analysis. This analysis demonstrated that samples primarily clustered based on GM, with a secondary clustering pattern based on genetic lineage (Fig. 3B). Notably, we found that certain metabolites had significant positive and negative correlations with adenoma number across all four rederived groups (Fig. 3C). A pathway analysis using putative metabolite identifications was performed to determine metabolic pathways modulated based on genetic lineage and GM colonization. Differential bile acid metabolism was observed when comparing $\mathrm{Min} / \mathrm{J}$ and $\mathrm{Min} / \mathrm{D}$ genetics, as defined by enrichment of putative bile acid intermediates (25R)-3 $\alpha, 7 \alpha$-dihydroxy-5 $\beta$-cholestanate and $3 \alpha, 7 \alpha, 12 \alpha$-trihydroxy-24oxo-5 $\beta$-cholestanoyl CoA in Min/D mice compared to Min/J (Table S5, Fig. 3D). Meanwhile, differential sphingosine lipid metabolism was observed based on GM colonization (Table S5). To summarize, a minority of differential features were specifically modulated by GM colonization or host genetic lineage, whereas the vast majority of features were modulated by a combination of the two factors. Furthermore, both individual metabolites and metabolic pathways were independently modulated based on genetic lineage or GM.

\subsubsection{Host genetic lineage influences bile acid metabolism}

The divergent genetic lineages $\mathrm{Min} / \mathrm{J}$ and $\mathrm{Min} / \mathrm{D}$ had significantly altered adenoma susceptibility and metabolic profiles. We therefore characterized genetic divergence between the $\mathrm{Min} / \mathrm{J}$ and $\mathrm{Min} / \mathrm{D}$ lines via $~ 30 \mathrm{X}$ whole-genome sequencing (WGS) on representative breeder female mice from each colony (see supplementary data and methods). SNPs and indels that were private to either $\mathrm{Min} / \mathrm{D}$ or $\mathrm{Min} / \mathrm{J}$ were 
categorized based on their predicted functional effect due to the nature of the variant using the Variant Effect Predictor (VEP) tool (Table S6). There were no private protein coding homozygous variants detected in either line, with all homozygous variants residing in noncoding regions. To interrogate overall effects of private mutations in each lineage, all private homozygous variants residing within or near known genes were used to identify over-represented KEGG (Kanehisa and Goto 2000) and REACTOME (JoshiTope, Gillespie et al. 2005) biological pathways using the over-representation tool in InnateDB, which revealed over-representation of bile-acid metabolism in the Min/D line (Table S8) (Breuer, Foroushani et al. 2013). Candidate genes with variants contributing to bile acid metabolism included Cyp39al, which codes for an enzyme involved in bile acid biosynthesis, and the intestinal bile acid transporter coded for by Fabp6 (Fuchs 2003, Ohmachi, Inoue et al. 2006). Notably, homozygous variants private to the Min/J line were detected near candidate genes $M y c$ and $D \lg 3$ among other cancer related genes (Table S7).

\subsubsection{The Min/D Fabp6 variant is associated with SI adenoma susceptibility}

Our WGS findings of variants associated with bile acid metabolism were particularly notable as they provide a possible explanation for the previously described changes in bile acid metabolites (Fig. 3D). However, it is unclear whether there are any functional consequences of the observed germline mutations. To determine whether detected Fabp6 and Cyp39al variants had potential downstream effects in the tissues they are normally expressed, we compared gene expression levels in the normal ileal epithelium and liver, respectively, of $\mathrm{Min} / \mathrm{J}$ and $\mathrm{Min} / \mathrm{D}$ mice. We found that Min/D mice had a significant reduction in Fabp6 expression in the ileal epithelium compared to $\mathrm{Min} / \mathrm{J}$ 
mice, while there were no differences in Cyp39al mRNA levels in the liver (Fig. 4B). We also evaluated expression of $M y c$ and $D \lg 3$ in ileal epithelium to determine the functional impact of variants associated with these genes. While there was substantial variability in $M y c$ expression, there were no significant differences in $M y c$ or $D \lg 3$ expression between the Min/J and Min/D lineages (Fig. S4A).

We validated the Fabp6 variant detected by WGS and further interrogated whether Fabp6 is a possible modifier of adenoma susceptibility. The Min/D and Min/J parental lines were used to generate N2 mice. Blinded tumor number assessment and correlation with the Fabp6 variants showed a significant association, where mice that were homozygous for the Min/D variant had the highest adenoma susceptibility. Those that were heterozygous displayed an intermediate phenotype, while mice that were homozygous for the Min/J variant had the lowest adenoma multiplicity (Fig. 4B). Thus, we observed differential Fabp6 expression between the Min/J and Min/D lineages associated with the validated upstream insertion in Min/D mice, and further associated this variant with SI adenoma susceptibility in N2 mice.

\subsubsection{Colonic adenoma susceptibility is associated with changes in bile acid metabolism}

We finally aimed to determine whether the fecal metabolome could account for the observed differences in colonic adenoma number between the original Min/D and Min/J colonies (Fig. 1A). An unsupervised dendrogram was generated to cluster the fecal metabolomes from 3 month old mice based on detected putative fecal metabolite features. The major root of the tree clustered samples into two distinct groups independent of genetic lineage and GM profile (Fig. 5A). Analysis of these two groups revealed that they 
correlated with colonic adenoma multiplicity, indicated by the numbers adjacent to the dendrogram, where one group had an average of $0.75 \pm 0.22$ colon adenomas, while the other had an average of $2.5 \pm 0.57$ colon adenomas. Linear Discriminant Analysis (LDA) was used to identify the associated with the low-adenoma and high-adenoma clusters. In total, we found 16 metabolites associated with the high-adenoma cluster, and 6 metabolites associated with the low-adenoma cluster (Fig. 5B). Of these metabolites, tandem MS enabled identification of two metabolites over-represented in the lowadenoma cluster, both of which were bile acid or bile acid derivatives. The relative abundance of putative cholate was primarily modulated by GM, while the abundance of putative $3 \beta, 7 \alpha, 12 \alpha$-Trihydroxy-5 $\alpha$-cholan-24-oic acid was dependent on both GM and genetic lineage (Fig. 5C). In conclusion, an unbiased clustering analysis of the fecal metabolomes of the rederived $A p c^{\text {Min }}$ groups generated two primary groups, which were associated with colonic adenoma numbers. Identification of two of these metabolites driving the low- and high-adenoma clusters revealed elevated levels of two bile acid compounds in the low-adenoma group, while the remainder are currently uncharacterized.

\subsection{Discussion}

The Min mouse has been the single most cited mouse model of human cancer for nearly three decades, yielding an extraordinary wealth of information about the pathogenesis and treatment of human disease. However, the use of the Min mouse model for quantitative analysis of tumor susceptibility and treatment has been confounded by phenotypic variability of unknown origin, particularly with respect to adenoma 
multiplicity (Table 1). Here, we demonstrate how leveraging the observed phenotypic variability between Min colonies allowed us to unravel the complex factors comprising disease susceptibility, with special focus on how host genetics and the gut microbiota (GM) collectively influence adenoma initiation. We utilized a multi-omics approach to integrate microbial community and host genomics data, and included the fecal metabolome to incorporate these data sets to provide new insight into the functional contributions of these interactions in CRC susceptibility.

We exploited our observation of a variable phenotype between two colonies that diverged from a common population; the original C57BL/6-Apc ${ }^{\text {Min }}$ colony generated and housed at the McCardle Laboratory at the University of Wisconsin (Min/D) and mice received from The Jackson Laboratory $(\mathrm{Min} / \mathrm{J})$ (Fig. 1A). Given the multi-generation segregation of the two colonies and the differences in selective pressures, we hypothesized that host genetic divergence would account for differences in adenoma susceptibility, despite having the same original inbred genetic background. Previous studies have also demonstrated that mice housed in different institutions have distinct GMs due to environmental differences, so we further hypothesized that the Min/D and Min/J colonies' disparate GM communities could contribute to distinct phenotypes (Ericsson, Davis et al. 2015). The original Min/D colony was rederived onto Hsd:CD1 surrogate dams from Envigo (previously Harlan) at the McCardle Laboratory, and therefore had a GM representing Envigo, while Min/J mice have a relatively less complex GM from The Jackson Laboratory (Hart, Ericsson et al. 2018). To segregate the effects of host genetics and GM on adenoma susceptibility, we used a unique targeted rederivation approach in which we generated mice of the Min/D and Min/J genetic lineages each with 
a GM representing either Envigo (GMHSD) or The Jackson Laboratory (GMJAX) (Fig. 1B). Remarkably, we demonstrated that both genetic lineage and GM considerably influenced adenoma numbers. While Min/J mice colonized with GMHSD had increased adenoma numbers compared to our original $\mathrm{Min} / \mathrm{J}$ colony, colonization of Min/D mice with GMJAX repressed adenoma numbers compared to the original Min/D colony, emphasizing a critical role for the GM in disease susceptibility in $A p c^{M i n}$ mice (Fig. 1C). Furthermore, rederived mice of the Min/D lineage developed more adenomas than their Min/J counterparts independent of GM colonization, indicating that genetic lineage similarly accounts for significant phenotypic variability (Fig. 1C). Thus, we demonstrate here that host genetics and the GM collectively accounted for the adenoma number disparity between two divergent colonies, additively determining adenoma multiplicity.

Microbial profiling via NGS of the $16 \mathrm{~S}$ rRNA gene allowed characterization of the GMJAX and GMHSD communities to identify OTUs associated with a protective versus deleterious phenotype. Analysis of $\beta$-diversity of the microbial taxa of GMHSD and GMJAX in the ileum and feces across multiple time points confirmed that these GMs were stably distinct from one another throughout the GI tract (Figs. 2 and S2).

Desulfovibrio sp. and Bilophila sp., which are deltaproteobacteria producers of hydrogen sulfide $\left(\mathrm{H}_{2} \mathrm{~S}\right)$ via sulfate and sulfite reduction, respectively, were 2-3 orders of magnitude higher in GMHSD compared to GMJAX in both ileal scrapes and feces (Tables S3 and S4) suggesting a potentially important role for sulfidogenic bacteria. A number of studies describe associations between $\mathrm{H}_{2} \mathrm{~S}$ and CRC risk, indicating both pro- and anticarcinogenic roles depending on concentration and route of cellular exposure (AtteneRamos, Wagner et al. 2006, Attene-Ramos, Wagner et al. 2007, Hellmich and Szabo 
2015, Guo, Yu et al. 2016). Of further interest, Bilophila sp., named for their close association with bile, is the only bacterial genera known to utilize taurine from taurineconjugated bile acids for anaerobic respiration and $\mathrm{H}_{2} \mathrm{~S}$ production (Laue, Friedrich et al. 2001, Carbonero, Benefiel et al. 2012). Due to its use of bile acids as a source of respiration, $B$. wadsworthia expands dramatically in western diets with higher fat content associated with increased taurine-conjugated bile acids, and thus presents a critical link between western diets, bile acid levels, sulfide production, and CRC risk (Ridlon, Kang et al. 2014). While these suggestive results remain correlative, experiments focused on supplementing these bacteria in an environmentally controlled setting could provide additional insight into complex community structures and their role in CRC pathogenesis.

The emergence of targeted and untargeted metabolomics provide an avenue to interrogate metabolic changes in disease. While the high sensitivity of an untargeted approach yields large numbers of metabolites of interest, distinguishing these compounds from unclassified fragments or adducts poses a significant challenge (Melnik, da Silva et al. 2017). This study in particular exemplifies the challenges of an untargeted approach, as the vast majority of detected differential metabolite features remain unidentified. It is also important to consider that more extensive annotation of certain metabolite classes may cause inherent bias when interpreting results. Thus, continued efforts to improve metabolite libraries, as well as bioinformatics pipelines that enable more efficient compound identification, are critical to the development of these approaches.

Despite these challenges, a wealth of information was gleaned from controlled metabolomics studies. The data show that both the GM and host genetics shape the fecal metabolome, and in the process, could alter predisposition to adenoma initiation (Fig. 
2A-B). Additional analyses enabled mapping of differential putative compounds to metabolic pathways, and thus show the perturbation of such metabolic pathways associated with pathology of interest. We identified dysregulation of bile acid metabolism in mice from the $\operatorname{Min} / \mathrm{D}$ genetic lineage. The enterohepatic BA system is a classic example of the inter-dependent nature of host genetics and the GM. Host gene expression of enzymes responsible for primary BA biosynthesis, as well as intestinal transporters that recycle these BAs are required for functional enterohepatic circulation (Thomas, Pellicciari et al. 2008), while the GM de-conjugates and transforms primary bile acids as they traverse the GI tract to produce secondary BAs. (Ridlon, Kang et al. 2006). Thus, intra-intestinal levels of BAs depend upon cooperative genomic and microbial functions.

Gleaning functional genomic significance of WGS variants is often especially challenging due to high numbers of misreads and unknown intergenic effects of poorly annotated functional elements. Thus, we used our metabolomics data, specifically identification of bile acid dysregulation, as a functional genomic tool to focus our variants of interest. We identified an insertion at a Spil transcription factor binding site of Fatty acid binding protein 6 (Fabp6), a protein responsible for the re-uptake of bile acids in the ileum for enterohepatic recirculation (Lefebvre, Cariou et al. 2009), in Min/D mice. This variant was associated with decreased expression of Fabp6 in the Min/D population, suggesting a functional role for the insertion. Previous studies have implicated Fabp6 in human CRC where it was over-expressed in cancerous tissue relative to normal tissue. Counterintuitively, higher expression levels of Fabp6 within tumors correlated with smaller tumors and less metastasis, suggesting its potential role in early carcinogenesis (Ohmachi, Inoue et al. 2006). Moreover, a western diet resulting in 
increased cell proliferation and tumorigenesis also significantly reduces FABP6 transporter levels, suggesting that the detected Fabp6 variant mimics the effects of a western diet as a modifier of disease susceptibility (Dermadi, Valo et al. 2017). To summarize, decreased expression of Fabp6 in normal ileal epithelium associated with increased adenomagenesis in Min/D mice, and segregation of the Min/D Fabp6 variant associated with SI adenoma multiplicity in N2 mice. Together, these results support the proposed role for Fabp6 in tumor initiation (Fig. 4B).

While it is difficult to discern contributing factors to colonic adenoma development in $A p c^{\mathrm{Min}}$ mice due to low tumor numbers and an incompletely penetrant phenotype, metabolomics provided a foundation for identifying changes associated with a more severe or suppressed colonic phenotype. An unbiased analysis of fecal metabolites in our rederived groups separated the metabolic profiles into two distinct groups defined by colonic tumor number. Among several associated compounds, we identified two bile acids whose increased abundance was associated with the low-adenoma group (Fig. 5). Previous studies have implicated secondary bile acids such as deoxycholic acid (DCA) in CRC pathogenesis through mechanisms including oxidative damage and mitochondrial dysfunction, while primary bile acids can inhibit adenoma formation (Smith, Keshavan et al. 2010, Degirolamo, Modica et al. 2011, Cao, Luo et al. 2014). Cholic acid (CA) is a primary bile acid converted to DCA by the gut microbiota (Brown, Rao et al. 2016). Enrichment of cholate in the low-adenoma group, and its association with GMJAXcolonized mice, may indicate that GMJAX converts CA to DCA less efficiently than GMHSD, and therefore confers a suppressed adenoma phenotype. These results highlight the diversity of bile acids and their potential effects on host cell proliferation in CRC, and 
suggest that carcinogenesis may depend upon a delicate balance between the two.

However, further targeted studies are required to better characterize the mechanism of dysregulation of primary and secondary bile acids, and to determine how genetic variants and the microbiota each influence these metabolites.

Colorectal cancer is a classic example of a multifaceted disease with complex biological systems contributing to overall susceptibility and pathogenesis. Here, we demonstrate that complex GM communities and host genetics both independently and additively modulate adenoma development in $A p c^{M i n}$ mice. We utilized a metabolomics platform to show that genetically divergent host genomes and complex GM interactively shape the intestinal metabolome. Our unique strategy of utilizing untargeted metabolomics data as a functional genomics tool enabled us to focus our attention to WGS variants of consequence. Thus, we demonstrate a novel tactic to extract pathologically relevant functional candidate variants from large cohorts of sequencing data. This work provides a platform for both mechanistic links between genetic variants and the GM as well as novel biomarker discovery, and supports the use of metabolomics for risk assessment of tumor susceptibility. Furthermore, this data provides a clear explanation for much of the variability observed in the $A p c^{\operatorname{Min}}$ tumor phenotype throughout its use over the course of several decades, and explains substantial differences in susceptibility to $\mathrm{CRC}$ across different human populations. Finally, this approach was relatively non-invasive and can be translated to human studies, integrating the complicated interactive nature of the host genome, the GM, and the fecal metabolome to create individualized risk assessment and tailored preventive medicine strategies. 


\subsection{Author Contributions and Acknowledgements}

Experiments were designed by JM and JAL with input from CF and LS. Wholegenome sequencing was performed by AD and TK. Metabolomics sample preparation, analyses, and processing were performed by JM and ZL. SB performed LefSe analyses and helped gavage the animals with the bacterial cultures. The authors would like to thank William Dove for generously donating the C57BL/6JMlcr-Apc ${ }^{M i n} / \mathrm{Mmmh}$ mice to the MMRRC for public distribution and comments on the manuscript. We would also like to acknowledge the contributions of Nathan Bivens and the MU DNA Core for assistance with 16S rRNA gene sequencing, Bill Spollen and the MU Informatics Research Core Facility for assistance with processing and analysis of $16 \mathrm{~S}$ rRNA sequencing data, and the MU Office of Animal Resources and their staff for assistance with animal husbandry and veterinary care.

\subsection{Financial Support}

This research was funded by grants from the National Institutes of Health to the Mutant Mouse Resource and Research Center at the University of Missouri (U42 OD010918), and by the University of Missouri to Dr. James Amos-Landgraf (Startupfunding). JM was supported by NIH T32 OD011126. Financial support for the MU Metabolomics Center and the Sumner lab was also provided by the University of Missouri. 


\subsection{Figures}

\section{Figure 1. Genetic lineage and GM colonization additively determine adenoma}

numbers in $\boldsymbol{A p c}^{\mathrm{Min}}$ mice. A, Scatter plots comparing mean ( \pm SEM) small intestinal (SI) and colon adenoma counts of the original B6- $A p c^{\text {Min }}$ colony generated at UW McArdle Laboratory $(\mathrm{Min} / \mathrm{D})$ to B6-Apc ${ }^{\mathrm{Min}}$ mice acquired from The Jackson Laboratory and maintained at University of Missouri $(\operatorname{Min} / \mathrm{J})(\operatorname{Min} / \mathrm{D}, \mathrm{n}=65 ; \operatorname{Min} / \mathrm{J}, \mathrm{n}=22)$. B, Embryos from the $\mathrm{Min} / \mathrm{J}$ and $\mathrm{Min} / \mathrm{D}$ genetic lineages were transplanted into surrogate dams harboring two distinct complex GM profiles; GMJAX and GMHSD. Offspring represent the two genetic lineages which have inherited a GM from their respective surrogate dams $\left(\operatorname{Min} / \mathrm{J}_{\mathrm{GMJAX}} n=13 ; \operatorname{Min} / \mathrm{D}_{\mathrm{GMJAX}} n=18 ; \operatorname{Min} / \mathrm{J}_{\mathrm{GMHSD}}, n=19 ; \operatorname{Min} / \mathrm{D}_{\mathrm{GMHSD}}, n=10\right) . \mathbf{C}$, Scatter plots comparing mean ( \pm SEM) SI and colon adenoma counts of the four rederived groups, including each genetic lineage (Min/J and Min/D) rederived with two complex GMs. ${ }^{*} p<0.05, * * p<0.01, * * * P<0.001$; Student's t-test (A) and Two-way ANOVA with the Student Newman-Keuls method (C). 
A
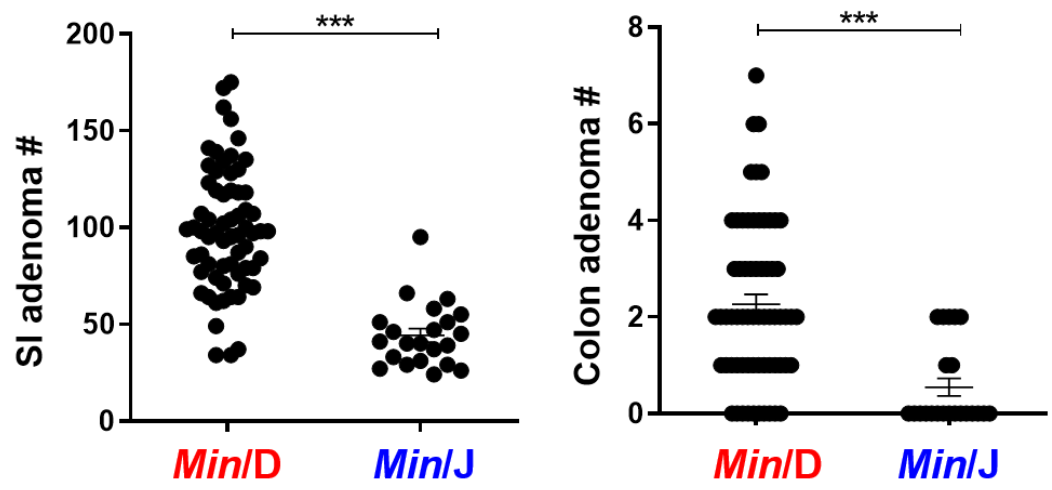

B

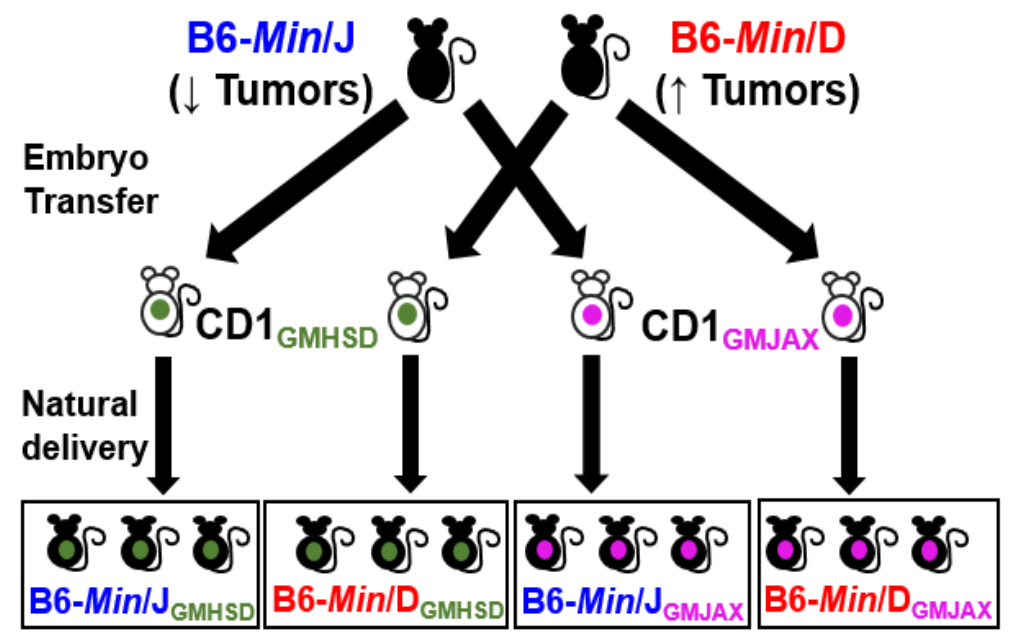

C
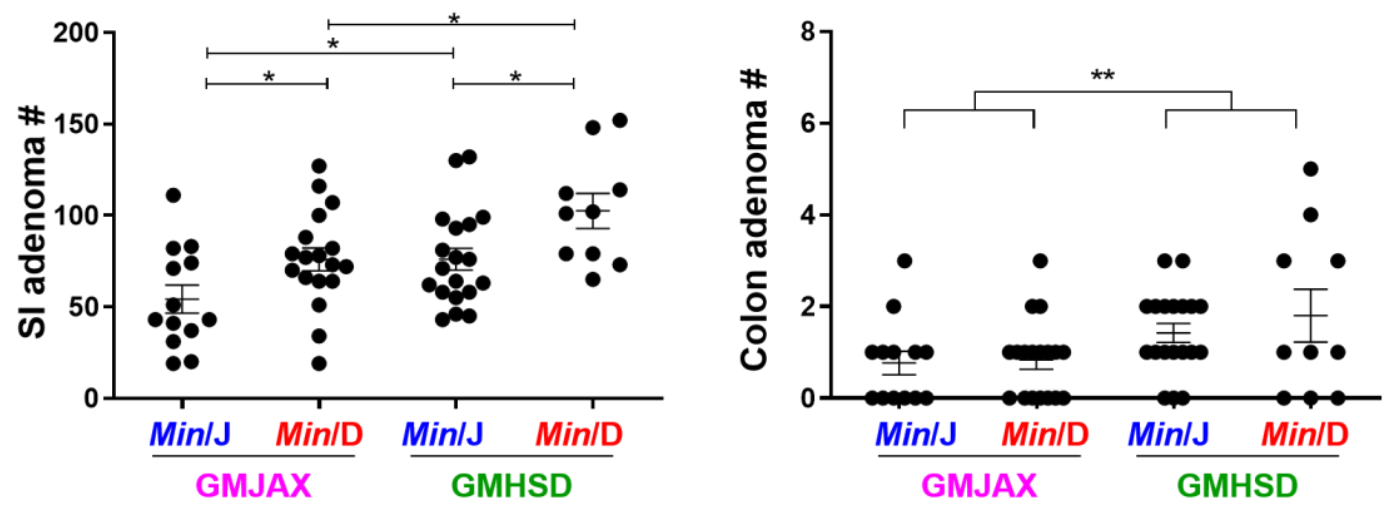
Figure 2. Distinct GM communities influence adenoma susceptibility. A, Bar charts representing relative abundances (mean \pm SEM) of Phyla with detected significant differences between fecal samples GMJAX and GMHSD groups $\left(\operatorname{Min} / \mathrm{J}_{\mathrm{GMJAX}}, n=13\right.$; $\left.\operatorname{Min} / \mathrm{D}_{\mathrm{GMJAX}}, n=18 ; \operatorname{Min} / \mathrm{J}_{\mathrm{GMHSD}}, n=19 ; \operatorname{Min} / \mathrm{D}_{\mathrm{GMHSD}}, n=10\right)$. B, Unweighted Principal Coordinate Analysis (PCoA) representing differences in $\beta$-diversity at the Operational Taxanomic Unit (OTU) level between complex GM profiles of CMTR offspring in feces at 1 month, and ileal scrapes at 3 months of age. C, Heatmap showing 25 taxa with significantly different $(p<0.001)$ fecal relative abundances between GMJAX and GMHSD at 1 month, where color intensity represents fold-change of each OTU. Hierarchical clustering based on Euclidean distances (top) demonstrates clustering of samples based on GM. All statistically significant OTUs and associated fold changes are represented in supplementary tables $3 \mathrm{~A}$ (fecal) and 3B (ileal). ${ }^{*} p<0.05, * * p<0.01, * * * p$ $<0.001$; Two-way ANOVA with the Student Newman-Keuls method for Multiple Comparisons.

A
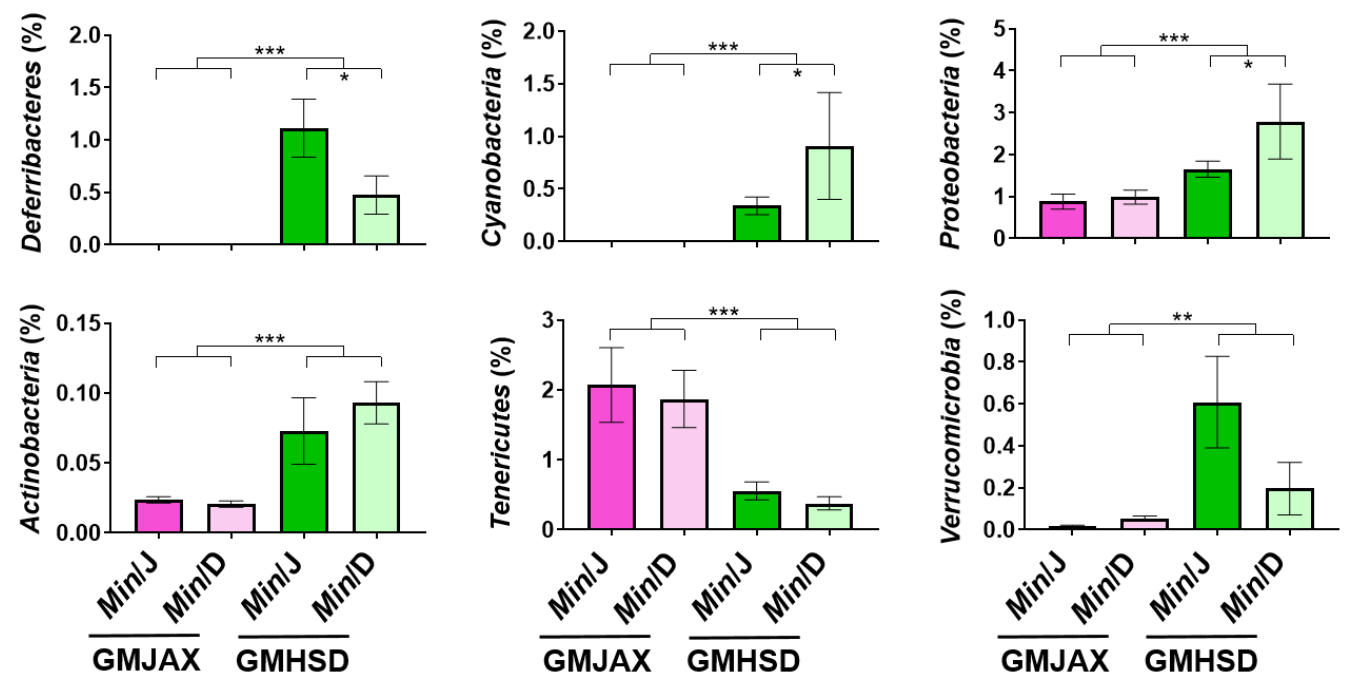
B
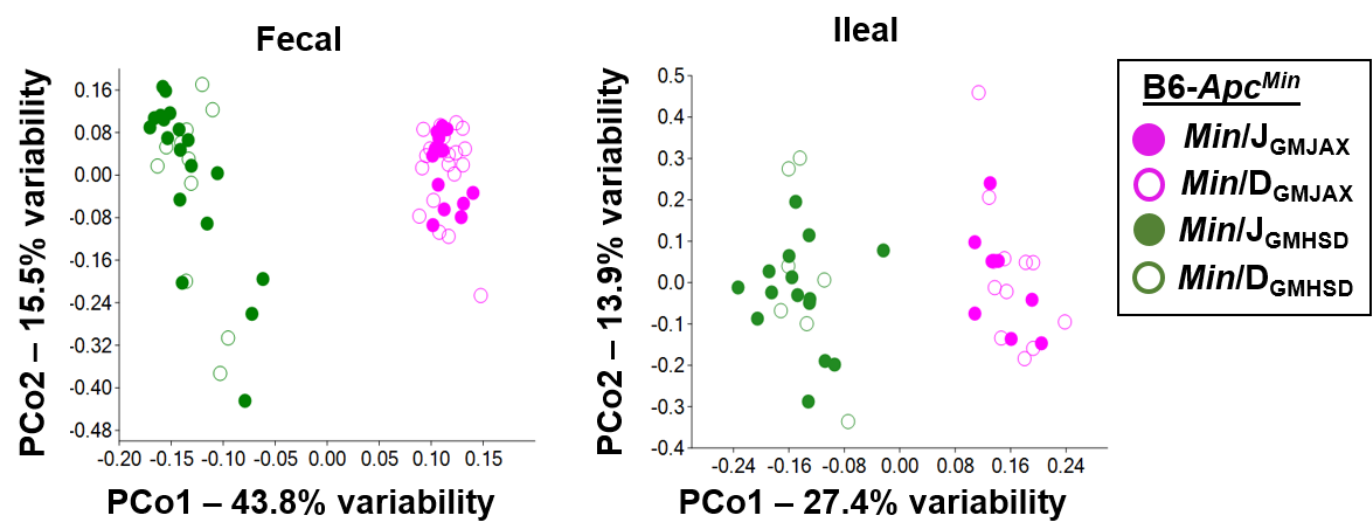

C

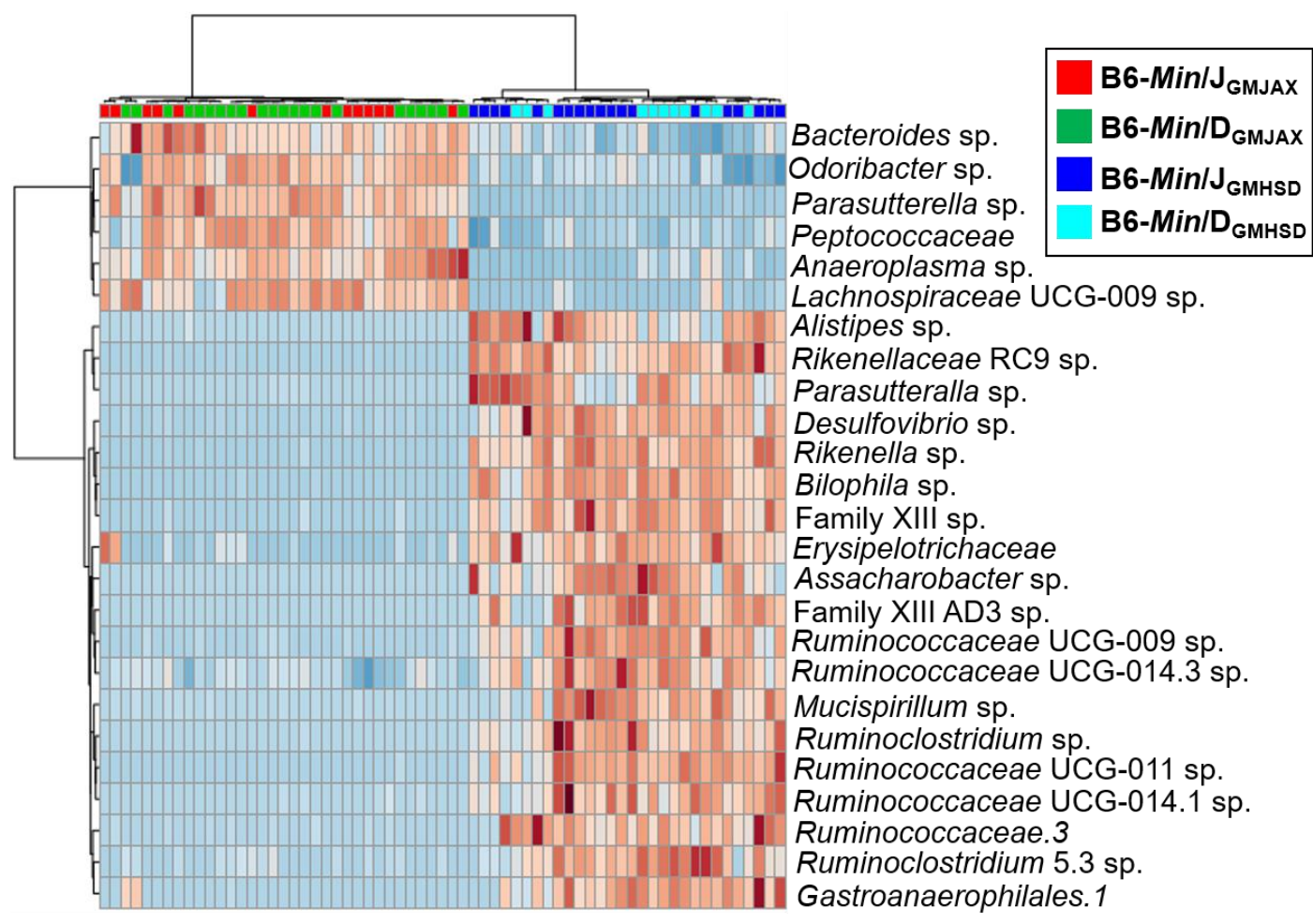




\section{Figure 3. Untargeted analysis of GM and host genetic lineage effects on the fecal}

metabolome. A, PCA illustrating unsupervised clustering of fecal metabolites at 3

months of age $\left(\operatorname{Min} / \mathrm{J}_{\mathrm{GMJAX}} n=6 ; \operatorname{Min} / \mathrm{D}_{\mathrm{GMJAX}}, n=4 ; \operatorname{Min} / \mathrm{J}_{\mathrm{GMHSD}}, n=5 ; \operatorname{Min} / \mathrm{D}_{\mathrm{GMHSD}}, n\right.$

=5). B, Heatmap showing 25 detected fecal metabolites with most significantly different relative abundances across all rederived groups, where color intensity represents foldchange of each metabolite. Hierarchical clustering based on Euclidean distances (top) illustrates primary clustering of samples based on GM, and secondary clustering based on genetic lineage. All metabolites shown on heat map have significantly different mean abundances $(p<0.001)$ based on ANOVA. C, Spearman's rank correlation was used to show metabolites with significant positive or negative correlations to SI tumor number across all rederived $A p c^{\text {Min }}$ groups $(\mathrm{n}=20)$. D, Scatter plots of mean \pm SEM relative abundances of putative metabolites contributing to modulation of bile acid metabolism $(\operatorname{Min} / \mathrm{J}, \mathrm{n}=6 ; \operatorname{Min} / \mathrm{D}, \mathrm{n}=4)$. Metabolites are denoted by mass-to-charge ratio and retention time $\left(\mathrm{m} / \mathrm{z}_{-} \mathrm{t}_{\mathrm{R}}\right) .{ }^{*} p<0.05, * * p<0.01, * * * p<0.001$; Student's t-test.

A

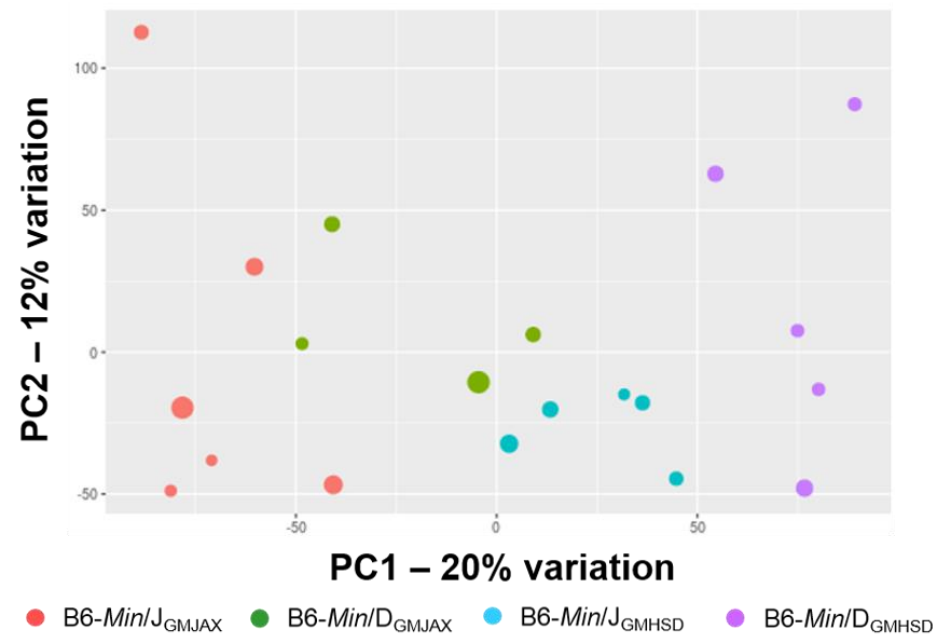


B

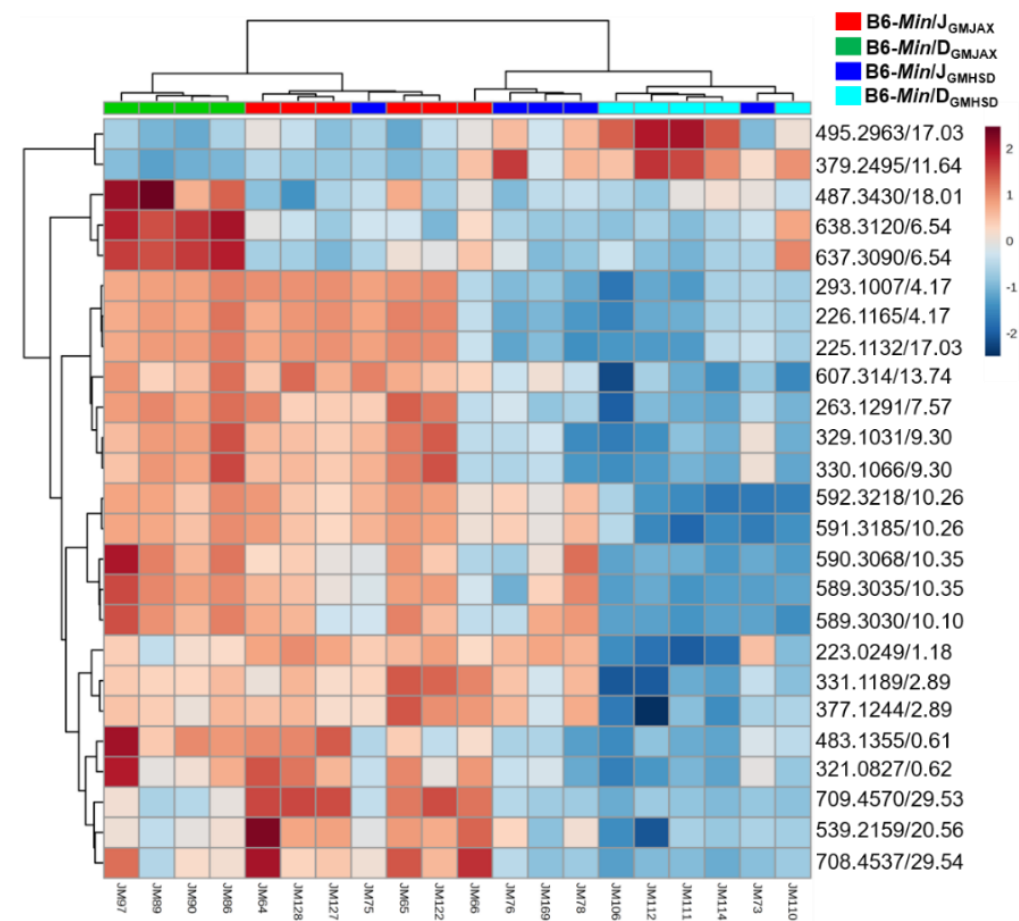

C
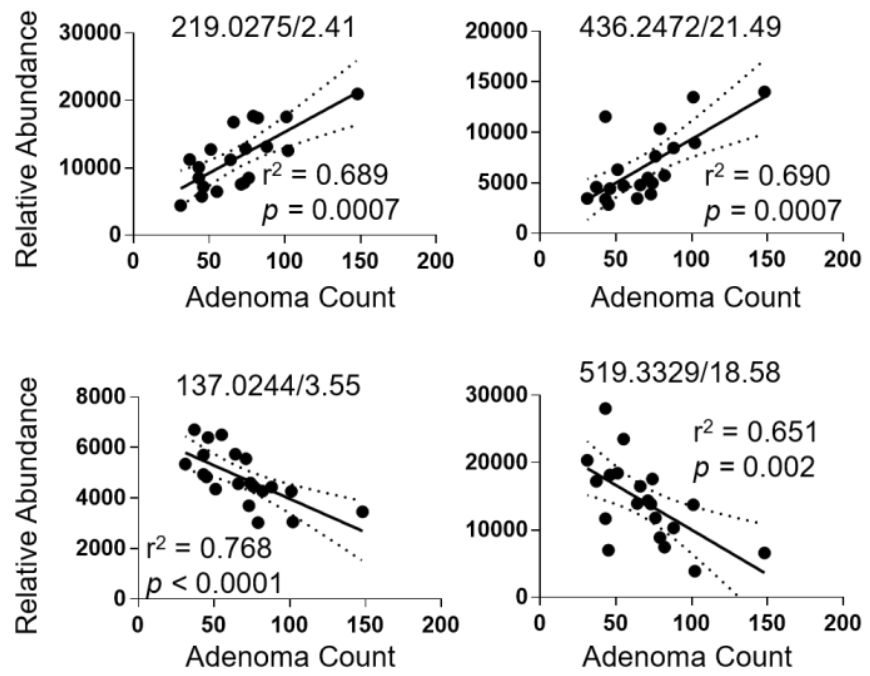

D

(25R)-3a,7 $\alpha$-dihydroxy$5 \beta$-cholestanate

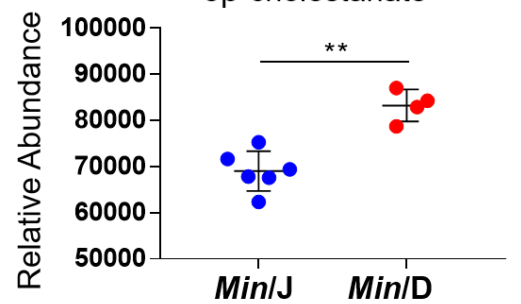

$3 \alpha, 7 \alpha, 12 \alpha$-trihydroxy-24oxo- $5 \beta$-cholestanoyl CoA

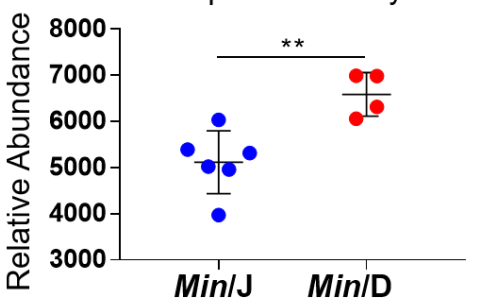


Figure 4. Min/D Fabp6 variant association with SI adenoma susceptibility. A, RTqPCR relative expression of candidate genes Fabp6 and Cyp39al in Min/J and Min/D lineages using ileal mucosal scrapes from normal intestinal epithelium and liver, respectively $(\operatorname{Min} / \mathrm{J}, \mathrm{n}=10 ; \operatorname{Min} / \mathrm{D}, \mathrm{n}=12)$. B, Scatter plots comparing mean $( \pm \mathrm{SEM}) \mathrm{SI}$ tumor counts of $\mathrm{N} 2$ mice based on status for the Fabp6 insertion (-/- homozygous for absence of insertion, $\mathrm{n}=29 ;+/$ - heterozygous for insertion, $\mathrm{n}=34 ;+/+$ homozygous for presence of insertion, $\mathrm{n}=16) .{ }^{*} p<0.05, * * p<0.01, * * * p<0.001$; Student's t-test (A) and ANOVA with the Student Newman-Keuls method (B).

A
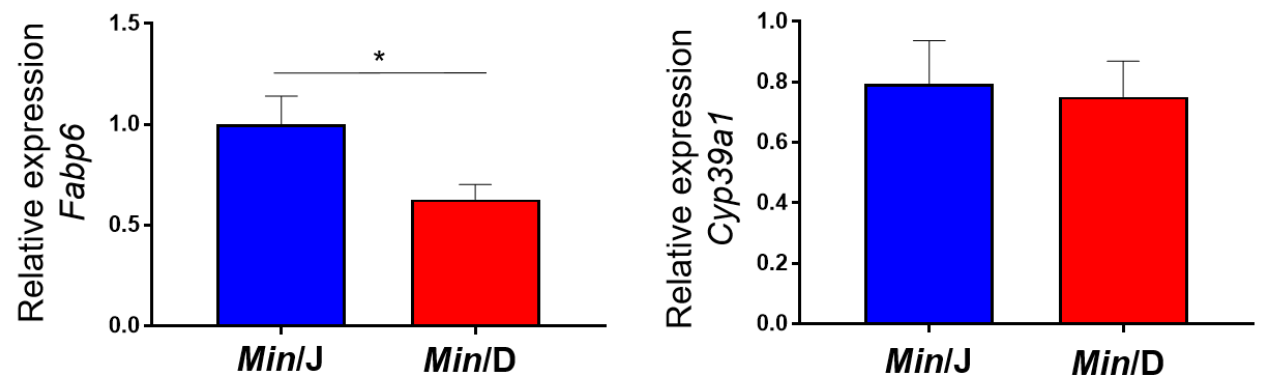

B

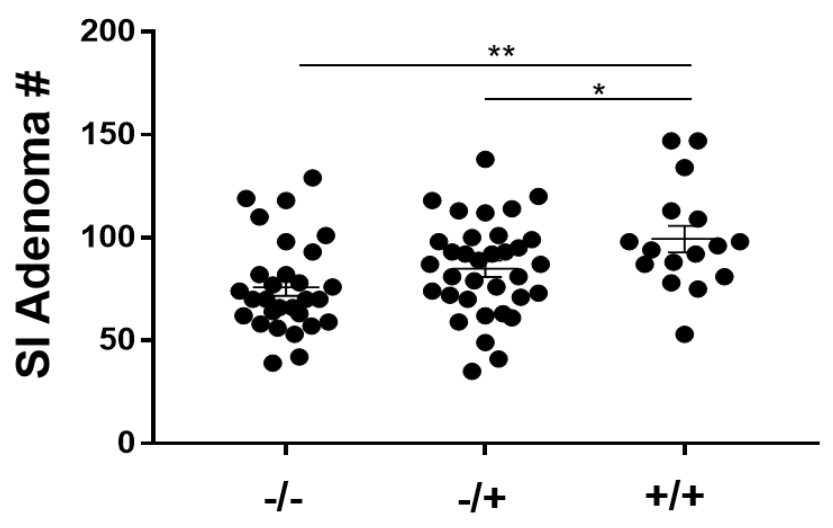

Fabp6 variant 
Figure 5. Colonic adenoma susceptibility is associated with changes in bile acid metabolism. A, Dendrogram was generated based on the putative fecal metabolite features using the Euclidean distance of measurement and Wards clustering algorithm. The major root of the tree clustered samples independent of genetic lineage and GM profile. The mean \pm SD is included for each cluster. B, Linear Discriminant Analysis (LDA) was used to identify the metabolites driving separation between the low-adenoma and high-adenoma clusters identified by the dendogram. C, Scatter plots displaying relative abundance of two bile acids identified by tandem MS, significantly overrepresented in the low-colonic adenoma group defined by the dendogram analysis $\left(\operatorname{Min} / \mathrm{J}_{\mathrm{GMJAX}}, n=6 ; \operatorname{Min} / \mathrm{D}_{\mathrm{GMJAX}}, n=4 ; \operatorname{Min} / \mathrm{J}_{\mathrm{GMHSD}}, n=5 ; \operatorname{Min} / \mathrm{D}_{\mathrm{GMHSD}}, n=4\right) .{ }^{*} p<0.05$, $* * p<0.01 ;$ student's t-test.

\section{A}

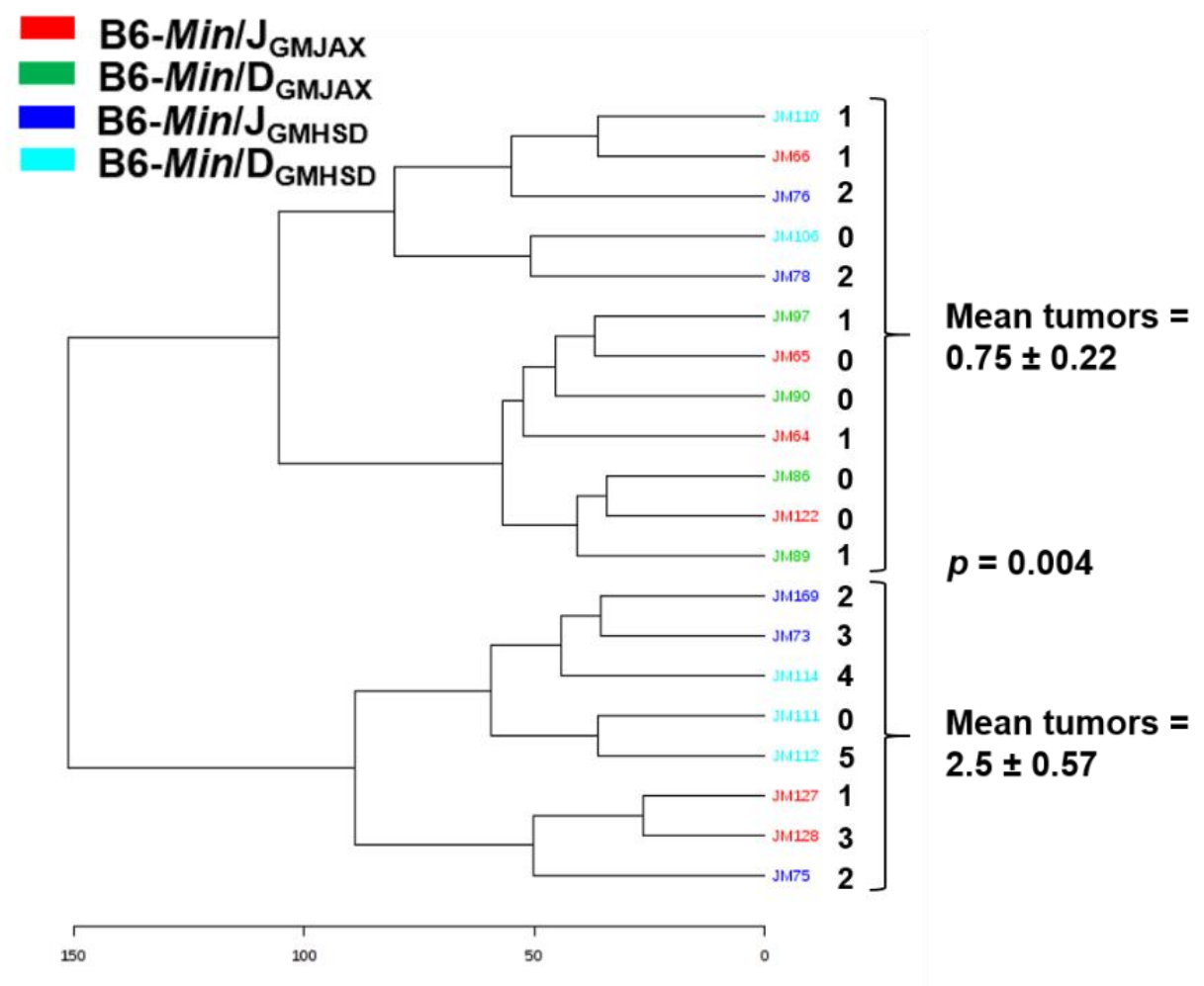


B
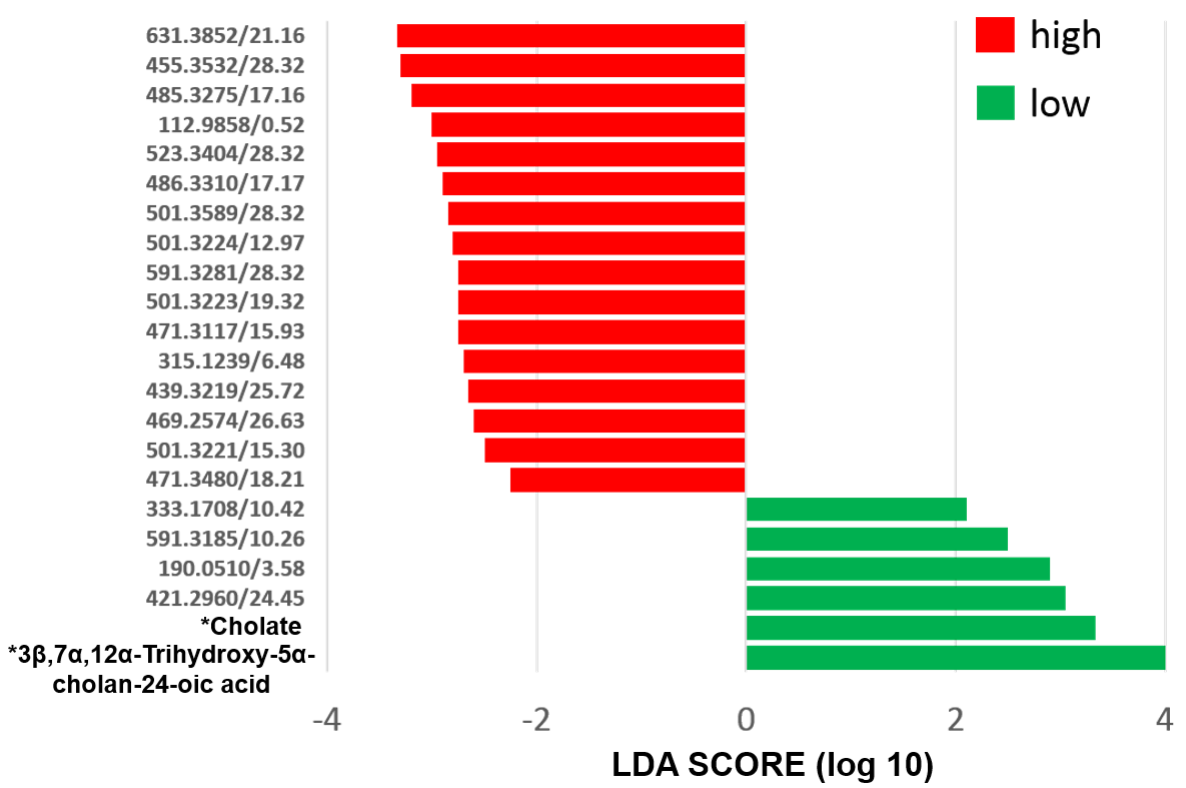

C

Cholate

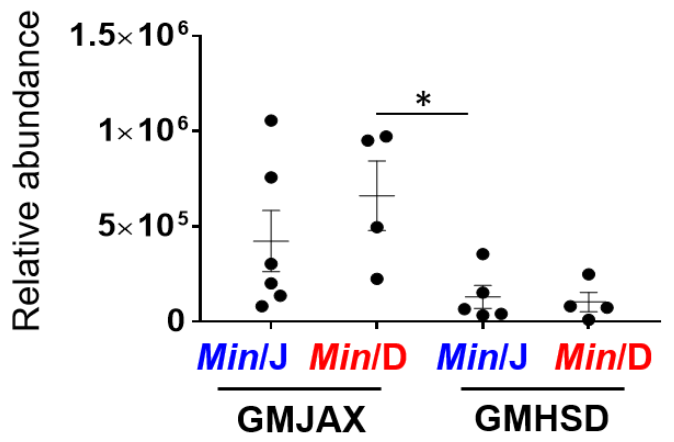

$3 \beta, 7 \alpha, 12 \alpha-T r i h y d r o x y-5 \alpha-$ cholan-24-oic acid

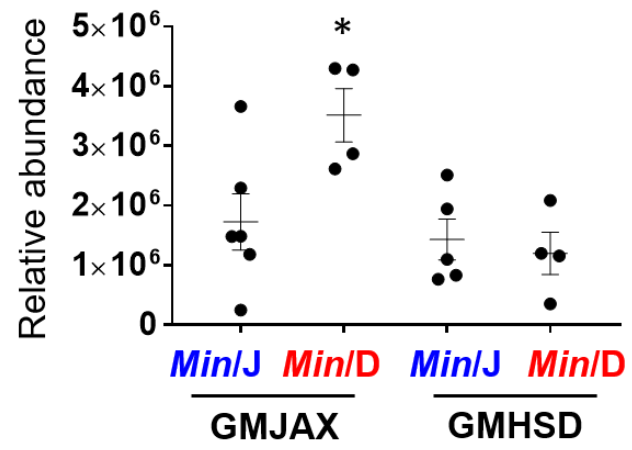




\section{Supplementary Figure 1. Effects of genetic lineage and GM on adenoma}

susceptibility by sex. Scatter plots comparing mean ( \pm SEM) small intestinal (SI) and colon adenoma counts of the original B6- $A p c^{\mathrm{Min}}$ colony generated at UW McArdle Laboratory $(\mathrm{Min} / \mathrm{D})$ to $\mathrm{B} 6-A p c^{\text {Min }}$ mice acquired from the Jackson Laboratory and maintained at University of Missouri $(\operatorname{Min} / \mathrm{J})$ of females $(\mathbf{A})(\operatorname{Min} / \mathrm{D}, \mathrm{n}=31 ; \operatorname{Min} / \mathrm{J}, \mathrm{n}=$ $3)$, and males (B) $(\operatorname{Min} / \mathrm{D}, \mathrm{n}=34 ; \operatorname{Min} / \mathrm{J}, \mathrm{n}=19)$. Scatter plots comparing mean $( \pm \mathrm{SEM})$ SI and colon adenoma counts of the four rederived groups, including each genetic lineage $(\operatorname{Min} / \mathrm{J}$ and $\operatorname{Min} / \mathrm{D})$ rederived with two complex GMs of females (C) $\left(\operatorname{Min} / \mathrm{J}_{\mathrm{GMJAX}}, n=4\right.$; $\left.\operatorname{Min} / \mathrm{D}_{\mathrm{GMJAX}}, n=9 ; \operatorname{Min} / \mathrm{J}_{\mathrm{GMHSD}}, n=10 ; \operatorname{Min} / \mathrm{D}_{\mathrm{GMHSD}}, n=4\right)$ and males $(\mathbf{D})\left(\operatorname{Min} / \mathrm{J}_{\mathrm{GMJAX}}, n\right.$ $\left.=9 ; \operatorname{Min} / \mathrm{D}_{\mathrm{GMJAX}}, n=9 ; \operatorname{Min} / \mathrm{J}_{\mathrm{GMHSD}}, n=9 ; \operatorname{Min} / \mathrm{D}_{\mathrm{GMHSD}}, n=6\right) .{ }^{*} p<0.05,{ }^{*} p<0.01$, *** $p<0.001$; student's t-test and Two-way ANOVA with the Student Newman-Keuls method.

A
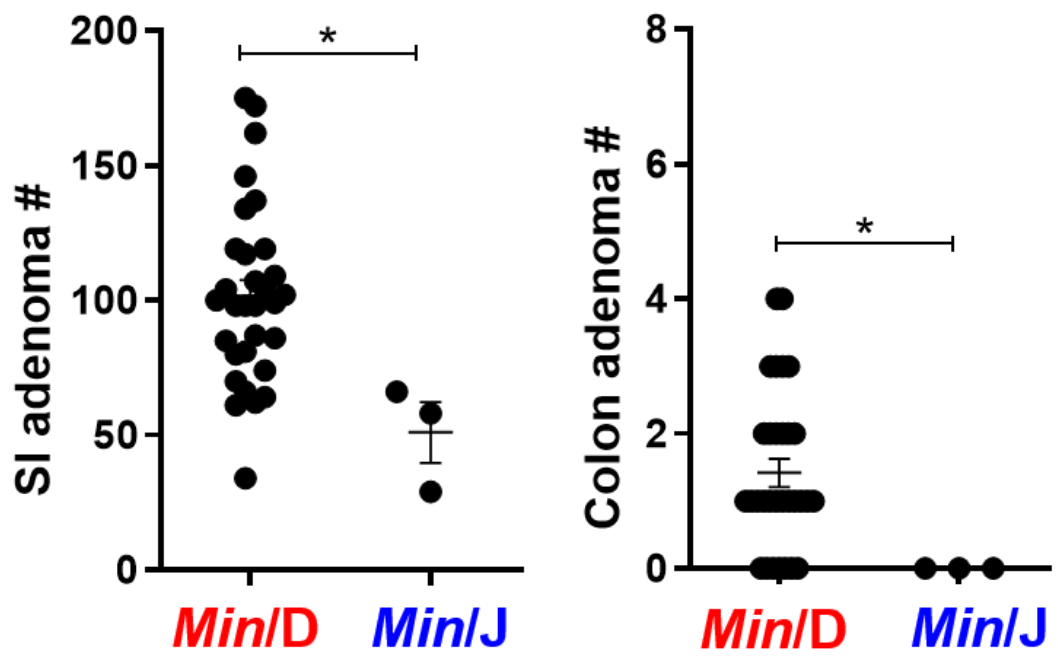
B
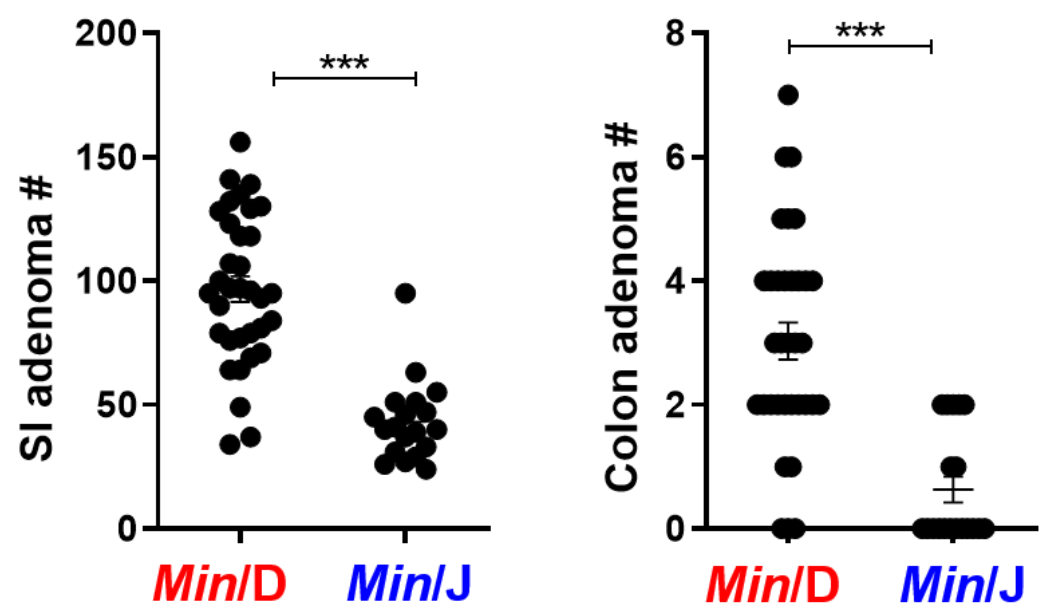

C
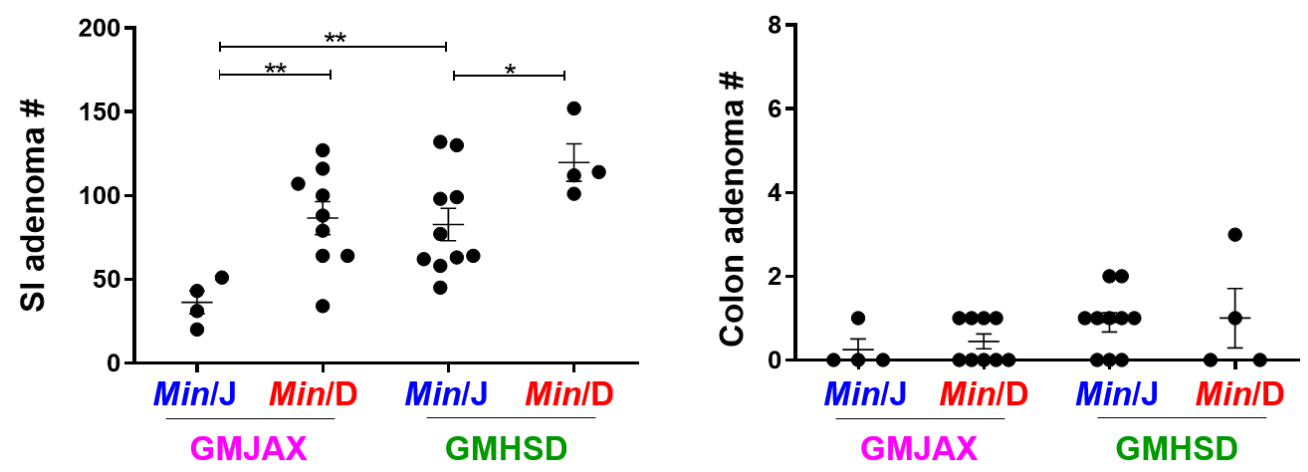

D
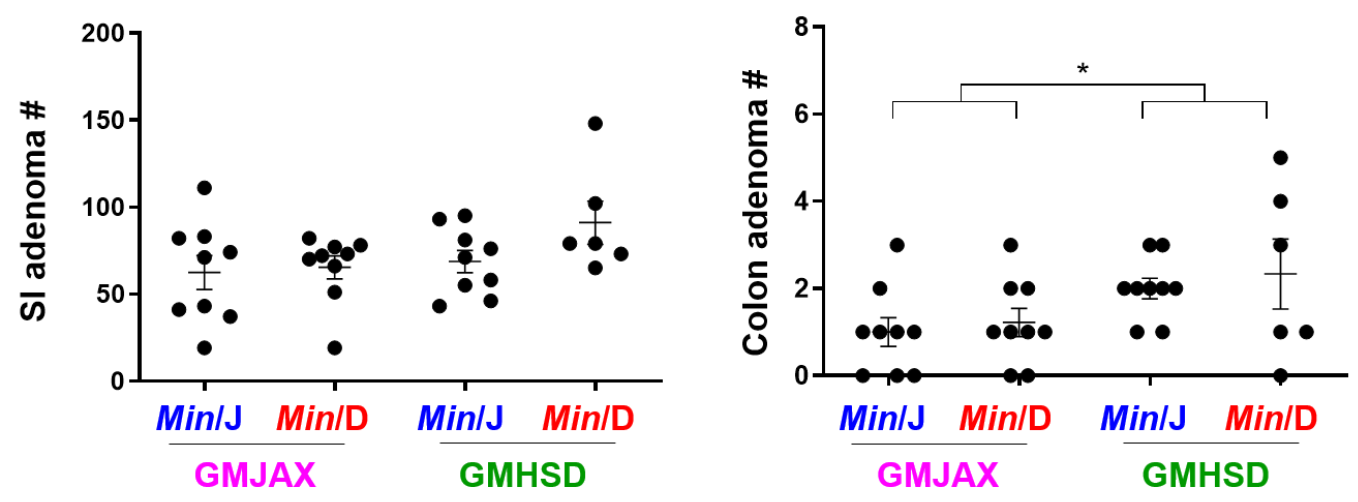


\section{Supplementary Figure 2. Analysis of $\alpha$ - and $\beta$-diversity measures of GMJAX and}

GMHSD. A, Unweighted PCoA representing differences in $\beta$-diversity at the Operational Taxanomic Unit (OTU) level between complex GM profiles of CMTR offspring in feces at 3 months of age and $\mathbf{B}$, unweighted PCoA of 1 month fecal OTUs separated by sex. $\mathbf{C}$, Differences in GM richness (Chaol index) and $\alpha$-diversity (Shannon Index) are shown with Tukey's boxplots. ${ }^{*} p<0.05, * * p<0.01, * * * p<0.001$; Kruskal-Wallis ANOVA on ranks with Dunn's Method for Multiple Comparisons.

A

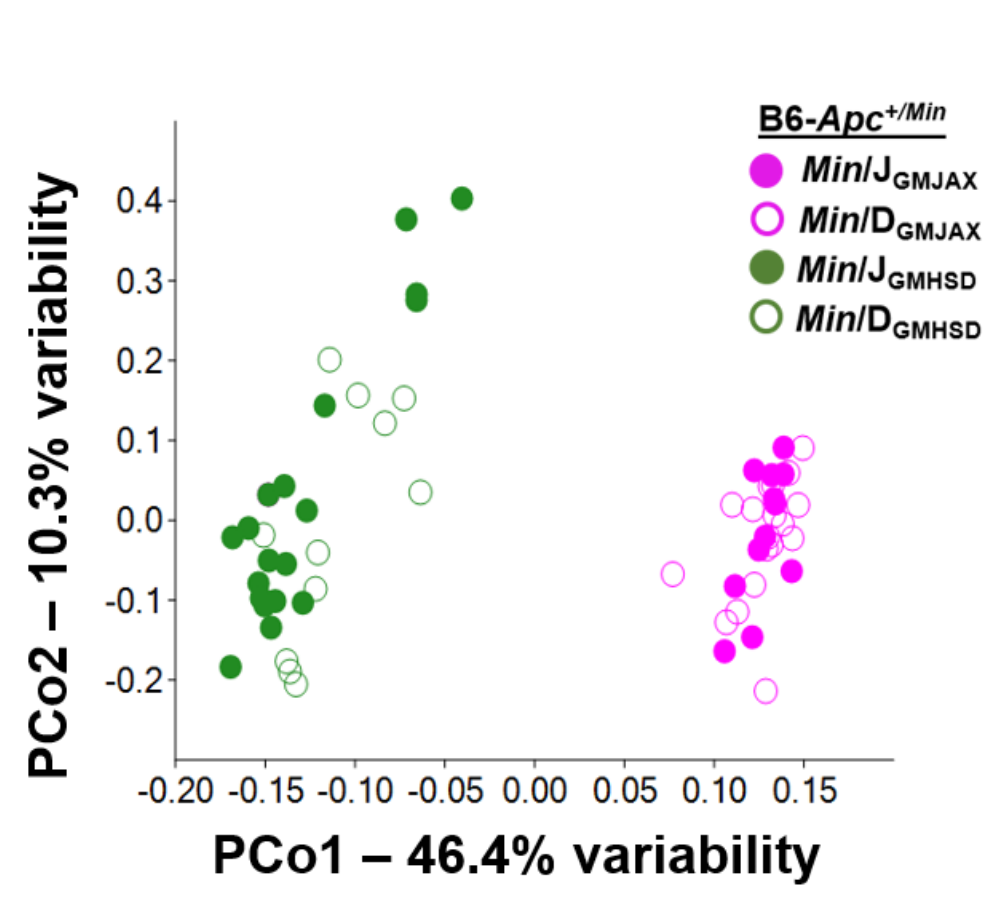


B

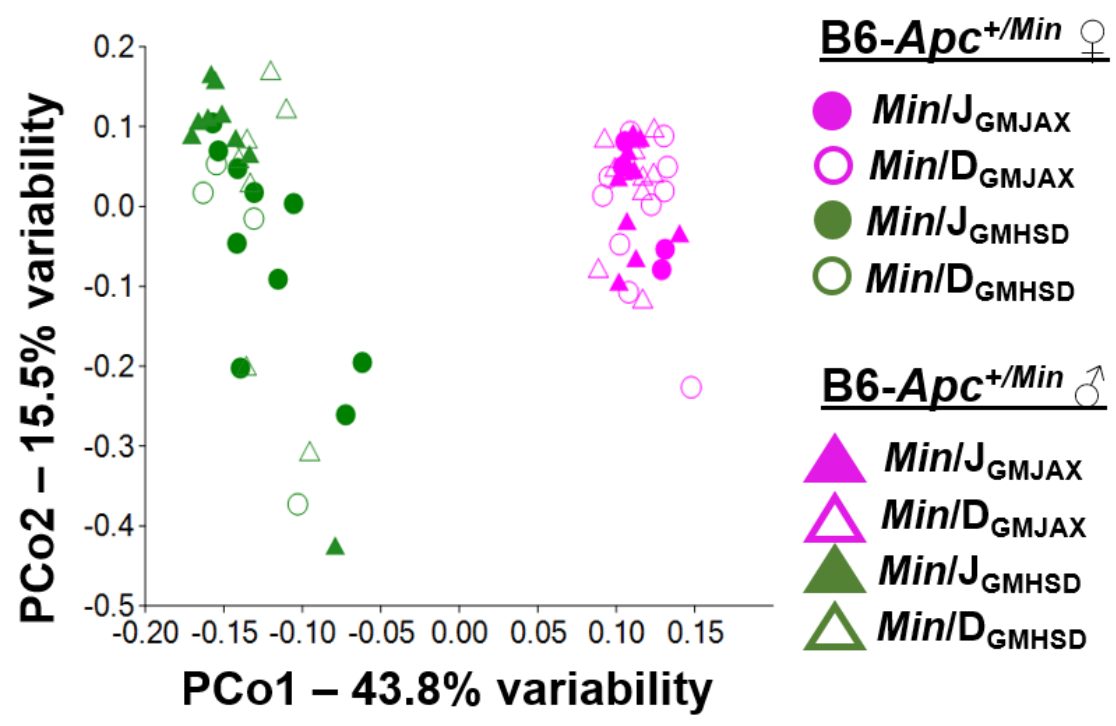

C
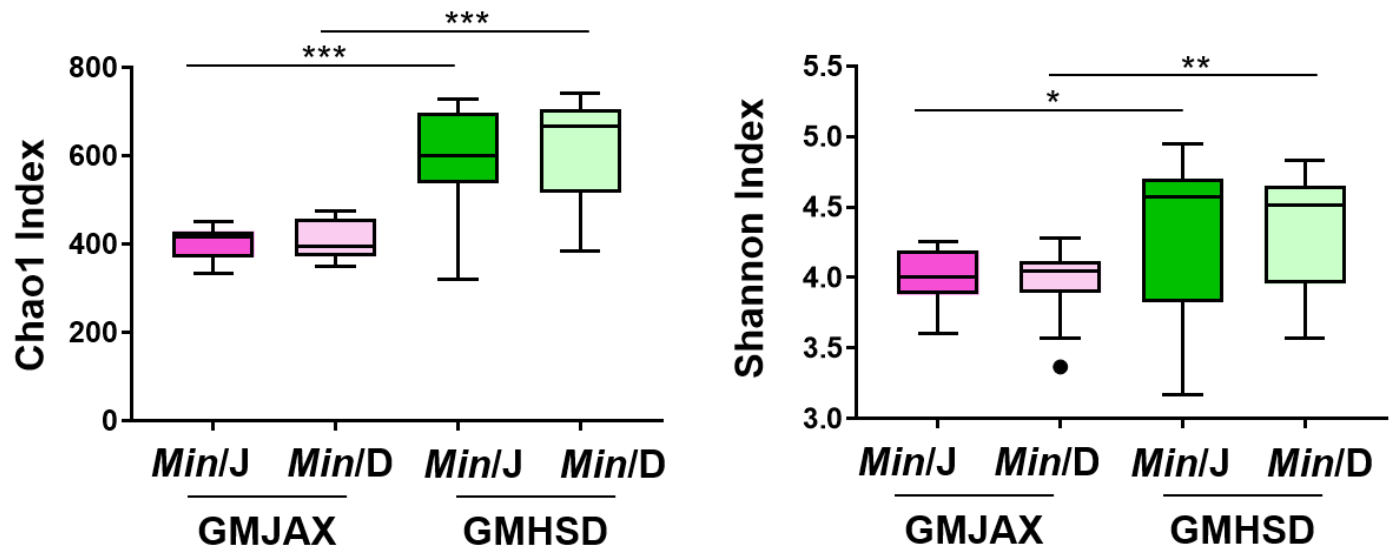


\section{Supplementary Figure 3. GM profile and genetic lineage modulate relative}

abundances of specific fecal metabolites. A, Scatter plots showing relative abundance of fecal metabolites modulated by the GM independent of genetic lineage (GMJAX, $n=$ 4; GMHSD, $n=5$ ). B, Scatter plots showing relative abundance of fecal metabolites modulated by genetic lineage independent of GM $(\operatorname{Min} / \mathrm{J}, \mathrm{n}=6 ; \operatorname{Min} / \mathrm{D}, \mathrm{n}=4)$. Metabolites are labeled by mass-to-charge ratio/retention time. $* * * p<0.001$.

A
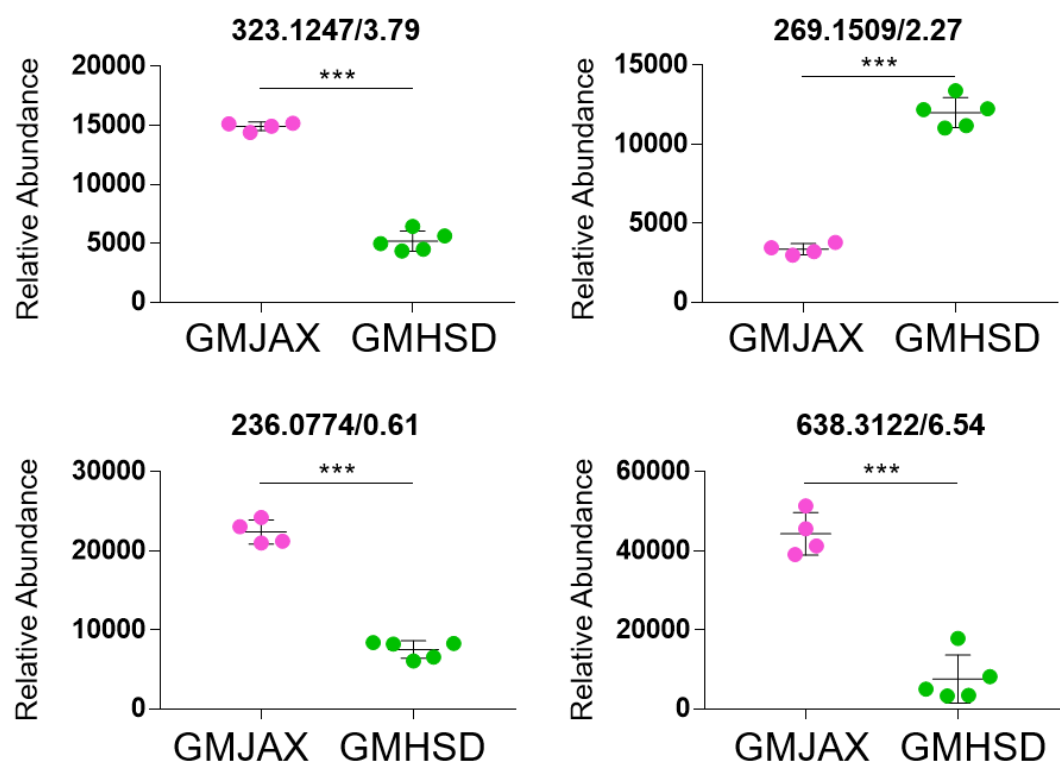

$638.3122 / 6.54$
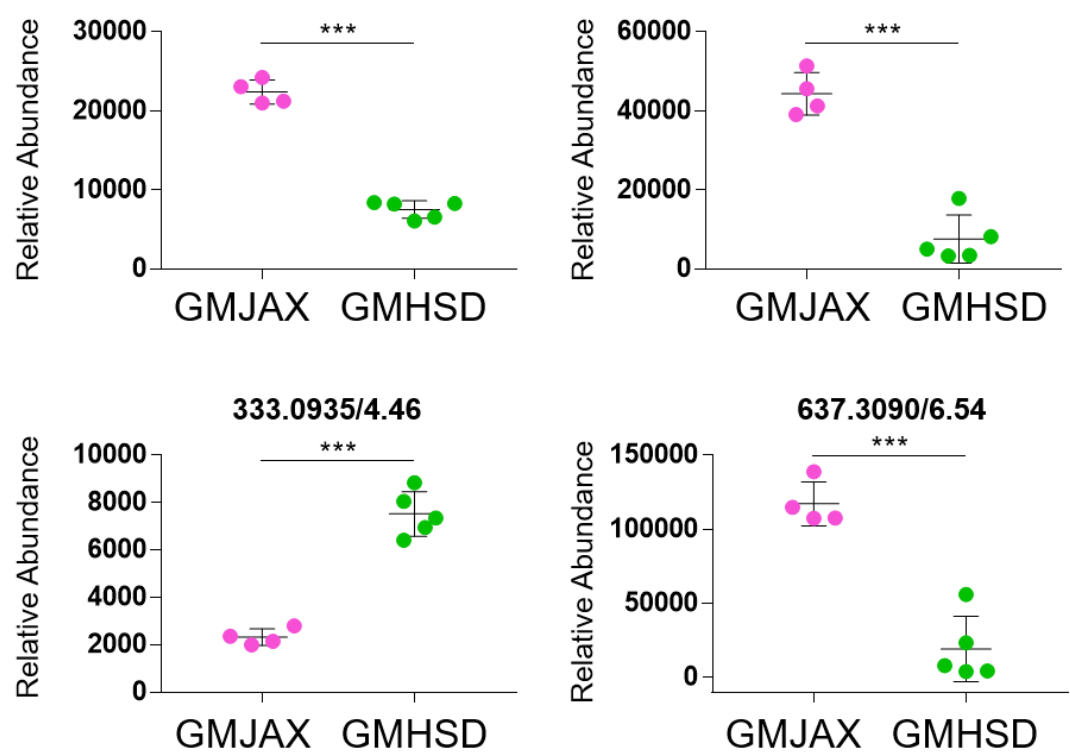
B
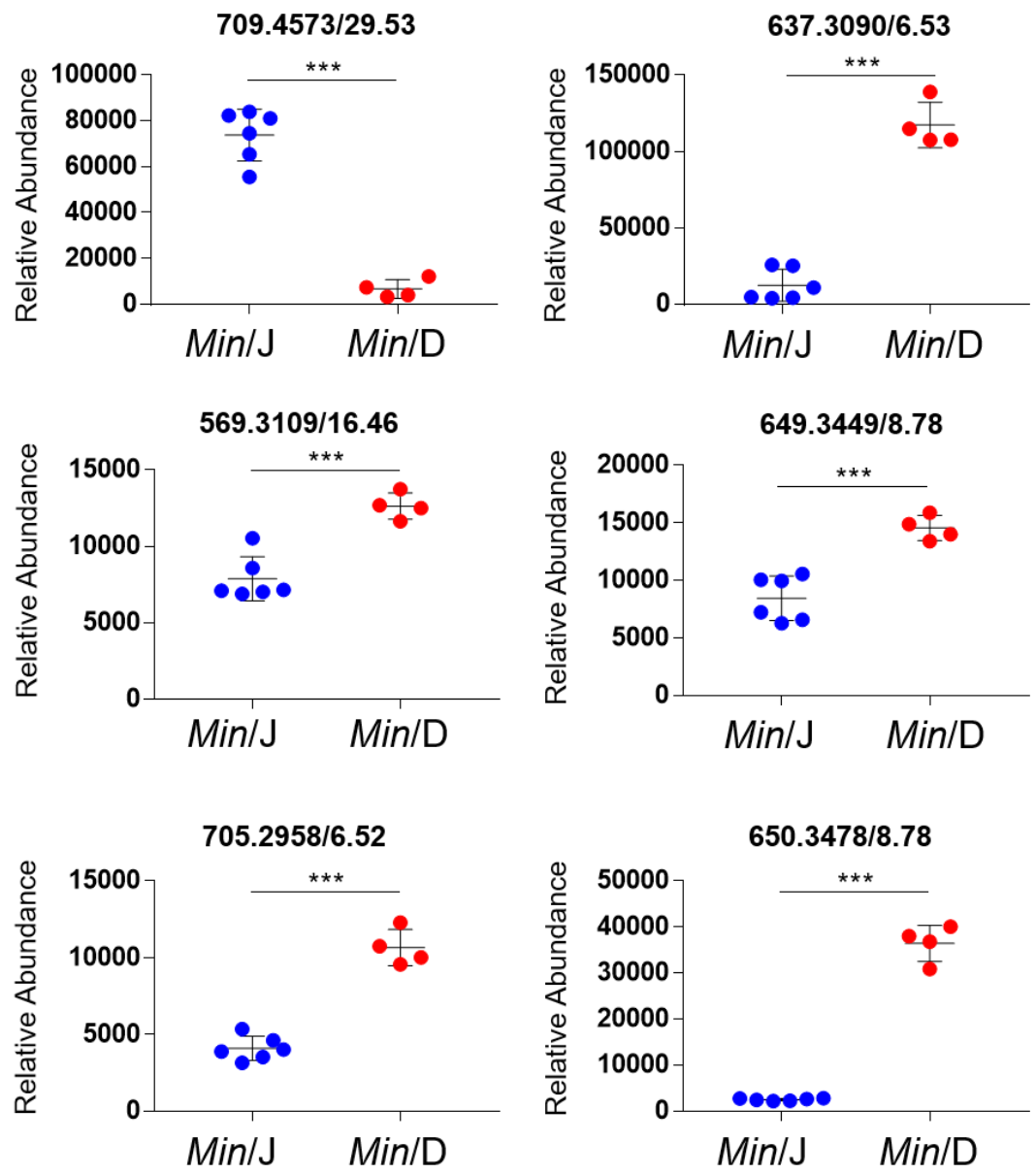
Supplementary Figure 4. Sequencing analysis of detected Fabp6 variant. A, RTqPCR comparison of relative expression ( \pm SEM) of candidate genes $M y c$ and $D \lg 3$ between $M i n / \mathrm{J}$ and $\operatorname{Min} / \mathrm{D}$ lineages $(\operatorname{Min} / \mathrm{J}, \mathrm{n}=6 ; \operatorname{Min} / \mathrm{D}, \mathrm{n}=6)$, using ileal mucosal scrapes from normal intestinal epithelium. B, Chromatogram of Sanger sequencing results for validation of the 5-bp deletion detected at position 43604912 of chromosome 11 in Min/J WGS. DNA extracted from ear punches of representative Min/D (top) and Min/J (bottom) mice, PCR amplified for the region of interest, and TA-cloned using the pCR 4-TOPO vector. The T7 sequencing primer (5'-TAATACGACTCACTATAGGG-3' was used for the sequencing reaction.

A
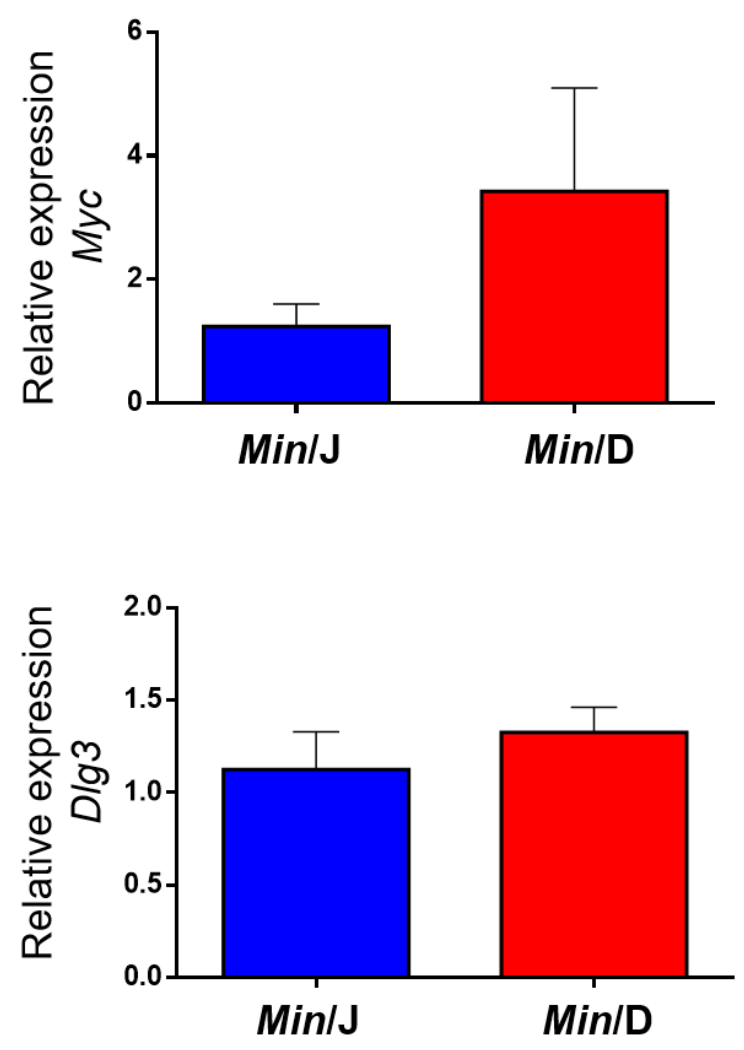
B
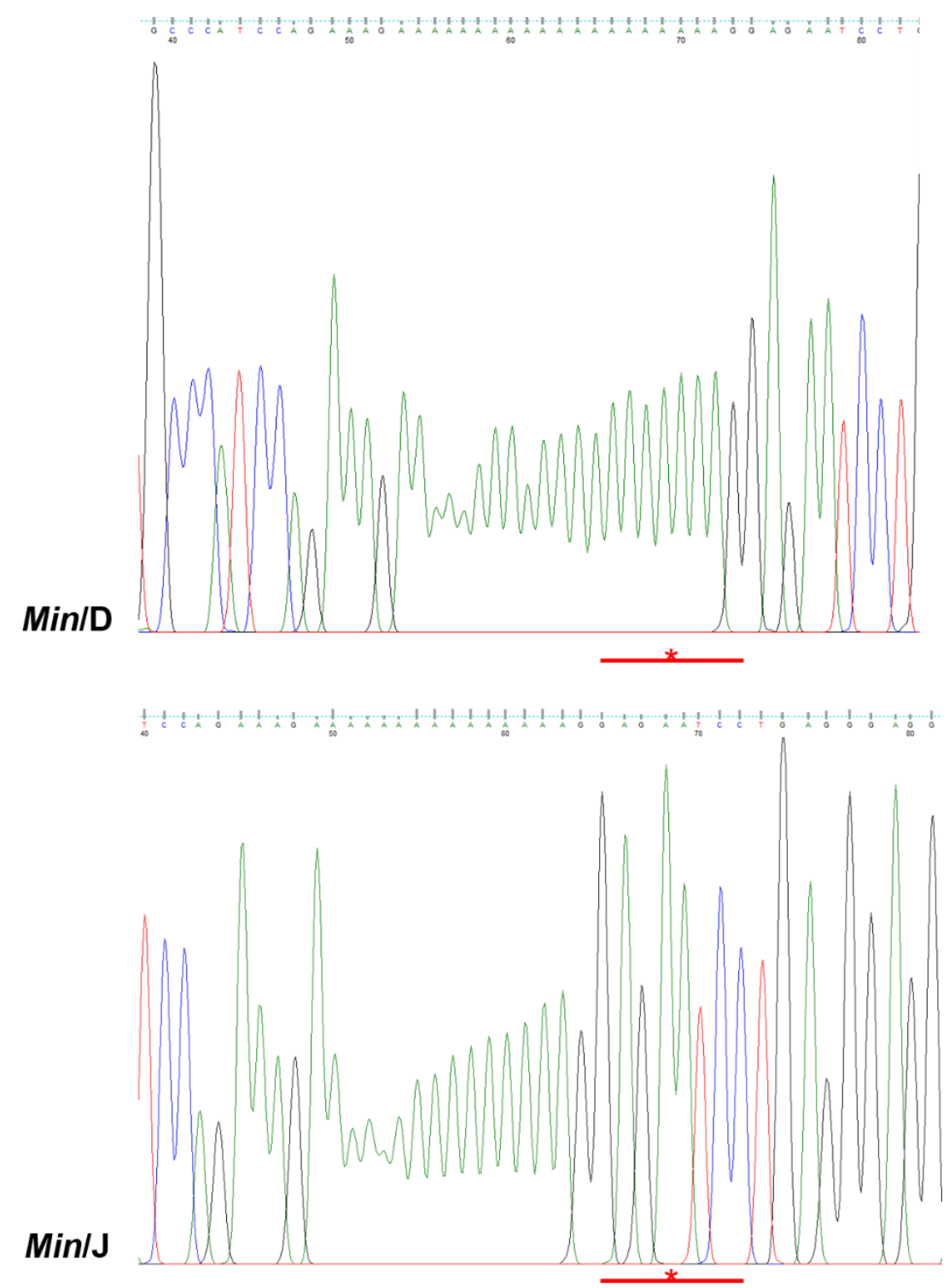


\section{Tables}

Table 1. Summary of small intestinal (SI) adenoma number variability between C57BL6/J-Apc $c^{M i n}$ colonies.

\begin{tabular}{|c|l|}
\hline $\begin{array}{c}\text { Tumor Count } \\
\text { (SI) }\end{array}$ & \multicolumn{1}{c|}{ Reference } \\
\hline 22 & MacGregor DJ et al. International Journal of Oncology. 2000. \\
\hline 34 & Zell JA et al. International Journal of Cancer. 2007. \\
\hline 41 & Chiu CH et al. Cancer Research. 1997. \\
\hline 71 & Niho N et al. Cancer Science. 2003. \\
\hline 102 & Ahn B and Ohshima H. Cancer Research. 2001. \\
\hline 108 & Paulsen JE et al. Carcinogenesis. 1997. \\
\hline 128 & Kwong et al. Genetics. 2007. \\
\hline
\end{tabular}


Supplementary table 1. Two-way PERMANOVA community analysis of fecal GM and host genetic effects on $\beta$-diversity

\begin{tabular}{|c|c|c|c|c|}
\hline & \multicolumn{2}{|c|}{ Bray Curtis } & \multicolumn{2}{c|}{ Jaccard } \\
\hline Variable & P value & $\boldsymbol{F}$ value & $\boldsymbol{P}$ value & $\boldsymbol{F}$ value \\
\hline GM (JAX vs HSD) & 0.0001 & 13.68 & 0.0001 & 43.26 \\
\hline Genetics (Min/J vs Min/D & .068 & 2.16 & 0.0025 & 4.36 \\
\hline Interaction & 0.716 & -5.18 & 0.85 & -5.23 \\
\hline
\end{tabular}

Bray Curtis and Jaccard's dissimilarity indices, based on 9999 permutations

Supplementary table 2. Two-way PERMANOVA Community analysis of ileal GM and host genetic effects on $\beta$-diversity

\begin{tabular}{|c|c|c|c|c|}
\hline & \multicolumn{2}{|c|}{ Bray Curtis } & \multicolumn{2}{c|}{ Jaccard } \\
\hline Variable & $\boldsymbol{P}$ value & $\boldsymbol{F}$ value & $\boldsymbol{P}$ value & $\boldsymbol{F}$ value \\
\hline GM (JAX vs HSD) & 0.0004 & 5.53 & 0.0001 & 9.46 \\
\hline Genetics (Min/J vs Min/D & .268 & 1.06 & 0.0020 & 2.43 \\
\hline Interaction & 0.758 & -5.36 & 0.378 & -4.18 \\
\hline
\end{tabular}

Bray Curtis and Jaccard's dissimilarity indices, based on 9999 permutations 
Supplementary table 3. Significantly different fecal OTUs between GMJAX and GMHSD

\begin{tabular}{|c|c|c|c|c|c|}
\hline \multicolumn{2}{|c|}{ Significant OTUs in B6-Apc $c^{+/ M i n}(p<0.001)$} & \multicolumn{2}{|c|}{$\begin{array}{c}\text { GMHSD } \\
\text { relative to GMJAX }\end{array}$} & \multirow{2}{*}{$\begin{array}{c}\text { GMJAX } \\
\text { Mean Relative } \\
\text { Abundance ( } \pm \text { SEM) }\end{array}$} & \multirow{2}{*}{$\begin{array}{c}\text { GMHSD } \\
\text { Mean Relative } \\
\text { Abundance }( \pm \text { SEM) }\end{array}$} \\
\hline Phylum & OTU & $p$-value & $\begin{array}{c}\text { Mean } \\
\text { Fold } \\
\text { Change }\end{array}$ & & \\
\hline Proteobacteria & Genus Bilophila & $2.18 \mathrm{E}-15$ & 523.12 & $7.29 \mathrm{E}-07 \pm 5.14 \mathrm{E}-07$ & $0.0025 \pm 0.0003$ \\
\hline Bacteroidetes & Genus Odoribacter & $2.19 \mathrm{E}-15$ & 685.34 & $2.95 \mathrm{E}-06 \pm 1.23 \mathrm{E}-06$ & $0.0044 \pm 0.0012$ \\
\hline Firmicutes & Family Peptococcaceae & $1.36 \mathrm{E}-14$ & 0.16577 & $0.0096 \pm 0.0007$ & $\begin{array}{c}0.0016 \\
\pm 0.0002 \\
\end{array}$ \\
\hline Bacteroidetes & Genus Rikenella & $3.31 \mathrm{E}-11$ & 662.83 & $2.92 \mathrm{E}-06 \pm 1.36 \mathrm{E}-06$ & $0.0043 \pm 0.0006$ \\
\hline Firmicutes & $\begin{array}{c}\text { Genus Ruminococcaceae } \\
\text { NK4A214 }\end{array}$ & $4.95 \mathrm{E}-11$ & 5.7893 & $0.0003 \pm 3.57 \mathrm{E}-05$ & $0.0015 \pm 0.0002$ \\
\hline Firmicutes & Genus Lachnospiraceae UCG-009 & $1.70 \mathrm{E}-10$ & 0.045779 & $0.0006 \pm 6.484 \mathrm{E}-05$ & $2.27 \mathrm{E}-05 \pm 1.23 \mathrm{E}-05$ \\
\hline Bacteroidetes & Genus Bacteroides & $8.19 \mathrm{E}-10$ & 0.26689 & $0.0918 \pm 0.0084$ & $0.0245 \pm 0.0025$ \\
\hline Firmicutes & Genus Family XIII AD3011 group & NA & $\mathrm{NA}$ & ND & $0.0001 \pm 1.95 \mathrm{E}-05$ \\
\hline Bacteroidetes & Parabacteroides goldsteinii & $2.46 \mathrm{E}-09$ & 0.28362 & $0.0019 \pm 0.0002$ & $0.0005 \pm 7.69 \mathrm{E}-05$ \\
\hline Firmicutes & Genus Ruminococcaceae UCG-009 & 3.49E-09 & 218.38 & $4.00 \mathrm{E}-07 \pm 4.00 \mathrm{E}-07$ & $0.0010 \pm 0.0002$ \\
\hline Proteobacteria & Genus Parasutterella & 4.54E-09 & 0.002419 & $2.04 \mathrm{E}-06 \pm 8.68 \mathrm{E}-07$ & $0.0024 \pm 0.0005$ \\
\hline Firmicutes & Genus Family XIII UCG-001 & $8.86 \mathrm{E}-09$ & 49.401 & $1.12 \mathrm{E}-06 \pm 6.32 \mathrm{E}-07$ & $0.0002 \pm 3.93 \mathrm{E}-05$ \\
\hline Firmicutes & Genus Ruminococcaceae UCG-014 & $2.84 \mathrm{E}-08$ & 10.455 & $0.0017 \pm 0.00021$ & $0.0182 \pm 0.0028$ \\
\hline Actinobacteria & Genus Asaccharobacter & NA & NA & ND & $6.78 \mathrm{E}-05 \pm 1.16 \mathrm{E}-05$ \\
\hline
\end{tabular}




\begin{tabular}{|c|c|c|c|c|c|}
\hline Firmicutes & Genus Lachnospiraceae UCG-010 & $1.09 \mathrm{E}-07$ & 0.15101 & $0.0003 \pm 3.92 \mathrm{E}-05$ & $4.43 \mathrm{E}-05 \pm 6.38 \mathrm{E}-06$ \\
\hline Firmicutes & Genus Ruminococcaceae UCG-014 & $1.43 \mathrm{E}-07$ & 364.11 & $3.68 \mathrm{E}-06 \pm 1.34 \mathrm{E}-06$ & $0.0026 \pm 0.0005$ \\
\hline Firmicutes & Family Peptococcaceae & $1.59 \mathrm{E}-07$ & 189.63 & $1.79 \mathrm{E}-06 \pm 9.52 \mathrm{E}-07$ & $0.0010 \pm 0.0002$ \\
\hline Bacteroidetes & Genus Fluviicola & NA & NA & ND & $0.0001 \pm 2.15 \mathrm{E}-05$ \\
\hline Bacteroidetes & Genus Rikenellaceae RC9 & 4.87E-07 & 1801.1 & $1.47 \mathrm{E}-06 \pm 7.21 \mathrm{E}-07$ & $0.0094 \pm 0.0018$ \\
\hline Bacteroidetes & Alistipes CC-5826 & $5.53 \mathrm{E}-07$ & 30.486 & $3.51 \mathrm{E}-07 \pm 3.51 \mathrm{E}-07$ & $0.0001 \pm 2.56 \mathrm{E}-05$ \\
\hline Firmicutes & Genus Ruminococcaceae UCG-003 & $7.59 \mathrm{E}-07$ & 55.968 & $1.70 \mathrm{E}-05 \pm 4.49 \mathrm{E}-06$ & $0.0011 \pm 0.0002$ \\
\hline Firmicutes & Family Clostridiales vadinBB60 & NA & NA & ND & $4.79 \mathrm{E}-05 \pm 3.10 \mathrm{E}-05$ \\
\hline Firmicutes & Genus Ruminiclostridium 5 & $1.02 \mathrm{E}-06$ & 60.332 & $9.00 \mathrm{E}-06 \pm 2.49 \mathrm{E}-06$ & $0.0007 \pm 0.0001$ \\
\hline Firmicutes & Family Erysipelotrichaceae & $1.25 \mathrm{E}-06$ & 8.3106 & $0.0001 \pm 3.36 \mathrm{E}-05$ & $3.44 \mathrm{E}-05 \pm 1.11 \mathrm{E}-05$ \\
\hline Proteobacteria & Genus Oxalobacter & $1.58 \mathrm{E}-06$ & 0.043163 & $0.0001 \pm 1.83 \mathrm{E}-05$ & $6.13 \mathrm{E}-07 \pm 6.13 \mathrm{E}-07$ \\
\hline Bacteroidetes & Genus Alloprevotella & $1.63 \mathrm{E}-06$ & 610.15 & $6.77 \mathrm{E}-06 \pm 1.45 \mathrm{E}-06$ & $0.0056 \pm 0.0011$ \\
\hline Proteobacteria & Genus Parasutterella & $1.80 \mathrm{E}-06$ & 427.03 & $0.0090 \pm 0.0012$ & $2.044 \mathrm{E}-05 \pm 3.65 \mathrm{E}-06$ \\
\hline Firmicutes & Family Christensenellaceae & $3.38 \mathrm{E}-06$ & 0.28271 & $0.0003 \pm 4.07 \mathrm{E}-05$ & $9.68 \mathrm{E}-05 \pm 2.31 \mathrm{E}-05$ \\
\hline Actinobacteria & Genus Senegalimassilia & NA & NA & ND & $1.41 \mathrm{E}-05 \pm 2.80 \mathrm{E}-06$ \\
\hline Firmicutes & Genus Christensenellaceae R-7 & $5.09 \mathrm{E}-06$ & 33.441 & $8.10 \mathrm{E}-07 \pm 4.56 \mathrm{E}-07$ & $0.0002 \pm 3.30 \mathrm{E}-05$ \\
\hline Tenericutes & Genus Anaeroplasma & $5.77 \mathrm{E}-06$ & 0.028716 & $0.0165 \pm 0.0030$ & $0.0005 \pm 0.0002$ \\
\hline Firmicutes & Genus Ruminiclostridium 5 & $7.04 \mathrm{E}-06$ & 0.13776 & $0.0120 \pm 0.0020$ & $0.0017 \pm 0.0002$ \\
\hline Deferribacteres & Genus Mucispirillum & $7.12 \mathrm{E}-06$ & 1078 & $5.15 \mathrm{E}-06 \pm 1.52 \mathrm{E}-06$ & $0.0088 \pm 0.0019$ \\
\hline Firmicutes & Genus Coprococcus & $1.06 \mathrm{E}-05$ & 0.34219 & $0.0054 \pm 0.0007$ & $0.0018 \pm 0.0003$ \\
\hline
\end{tabular}




\begin{tabular}{|c|c|c|c|c|c|}
\hline Cyanobacteria & Order Gastranaerophilales & $1.38 \mathrm{E}-05$ & 725.14 & $2.32 \mathrm{E}-06 \pm 8.83 \mathrm{E}-07$ & $0.0042 \pm 0.0020$ \\
\hline Proteobacteria & Genus Desulfovibrio & $1.55 \mathrm{E}-05$ & 1566.8 & $7.18 \mathrm{E}-06 \pm 1.89 \mathrm{E}-06$ & $0.0154 \pm 0.0036$ \\
\hline Firmicutes & Genus Ruminococcaceae UCG-013 & NA & NA & ND & $4.12 \mathrm{E}-05 \pm 9.25 \mathrm{E}-06$ \\
\hline Firmicutes & Genus Ruminococcaceae UCG-005 & $2.45 \mathrm{E}-05$ & 0.27122 & $0.0012 \pm 0.0002$ & $0.0003 \pm 5.42 \mathrm{E}-05$ \\
\hline Firmicutes & Genus Erysipelatoclostridium & $3.64 \mathrm{E}-05$ & 38.155 & $8.46 \mathrm{E}-07 \pm 5.90 \mathrm{E}-07$ & $0.0002 \pm 4.3734 \mathrm{E}-05$ \\
\hline Firmicutes & Catabacter hongkongensis & NA & NA & ND & $3.06 \mathrm{E}-05 \pm 7.15 \mathrm{E}-06$ \\
\hline Firmicutes & Genus Family XIII AD3011 group & $4.15 \mathrm{E}-05$ & 0.49768 & $0.0003 \pm 2.19 \mathrm{E}-05$ & $0.0001 \pm 1.75 \mathrm{E}-05$ \\
\hline Firmicutes & Genus Ruminococcaceae UCG-011 & $4.46 \mathrm{E}-05$ & 313.95 & $3.12 \mathrm{E}-06 \pm 1.04 \mathrm{E}-06$ & $0.0020 \pm 0.0005$ \\
\hline Tenericutes & Order Mollicutes RF9.1 & NA & NA & ND & $0.0002 \pm 4.80 \mathrm{E}-05$ \\
\hline Firmicutes & Genus Anaerovorax & 7.77E-05 & 0.33895 & $0.0001 \pm 1.46 \mathrm{E}-05$ & $4.82 \mathrm{E}-05 \pm 1.76 \mathrm{E}-05$ \\
\hline Firmicutes & Family Christensenellaceae & 0.0001 & 2.5038 & $2.75 \mathrm{E}-05 \pm 3.87 \mathrm{E}-06$ & $7.02 \mathrm{E}-05 \pm 1.05 \mathrm{E}-05$ \\
\hline Firmicutes & Family Lachnospiraceae & 0.000101 & 5.0579 & $0.0005 \pm 8.42 \mathrm{E}-05$ & $0.0024 \pm 0.0005$ \\
\hline Firmicutes & Genus Ruminiclostridium 5 & 0.00018 & 3.2504 & $7.05 \mathrm{E}-05 \pm 1.50 \mathrm{E}-05$ & $0.0002 \pm 3.97 \mathrm{E}-05$ \\
\hline Bacteroidetes & Genus Prevotella & 0.000218 & 0.46679 & $0.0067 \pm 0.0008$ & $0.0031 \pm 0.0004$ \\
\hline Bacteroidetes & Genus Odoribacter.1 & 0.000236 & 0.1121 & $0.0097 \pm 0.0008$ & $0.0011 \pm 0.0002$ \\
\hline Firmicutes & Family Ruminococcaceae.3 & 0.000283 & 186.66 & $2.64 \mathrm{E}-06 \pm 1.26 \mathrm{E}-06$ & $0.0012 \pm 0.0003$ \\
\hline Firmicutes & Family Ruminococcaceae.2 & NA & NA & ND & $1.54 \mathrm{E}-05 \pm 4.09 \mathrm{E}-06$ \\
\hline Firmicutes & Family Lachnospiraceae.4 & NA & NA & ND & $1.86 \mathrm{E}-05 \pm 5.07 \mathrm{E}-06$ \\
\hline Firmicutes & Family Lachnospiraceae.2 & 0.000373 & 415.54 & $1.59 \mathrm{E}-06 \pm 9.53 \mathrm{E}-07$ & $0.0023 \pm 0.0006$ \\
\hline Bacteroidetes & Genus Bacteroides.1 & 0.00038 & 0.21468 & $0.0001 \pm 1.97 \mathrm{E}-05$ & $2.07 \mathrm{E}-05 \pm 7.15 \mathrm{E}-06$ \\
\hline
\end{tabular}




\begin{tabular}{|c|c|c|c|c|c|}
\hline Firmicutes & Genus Ruminiclostridium & 0.000385 & 527.2 & $3.97 \mathrm{E}-07 \pm 3.97 \mathrm{E}-07$ & $0.0024 \pm 0.0007$ \\
\hline Firmicutes & $\begin{array}{c}\text { Genus Ruminococcaceae UCG- } \\
\text { 005.1 }\end{array}$ & NA & NA & ND & $6.99 \mathrm{E}-05 \pm 1.99 \mathrm{E}-05$ \\
\hline Firmicutes & bacterium NLAE-zl-H60 & 0.000733 & 0.15777 & $0.0018 \pm 0.0004$ & $0.0003 \pm 7.82 \mathrm{E}-05$ \\
\hline Firmicutes & Genus Peptoclostridium & 0.000814 & 0.061672 & $0.0014 \pm 0.0004$ & $8.67 \mathrm{E}-05 \pm 3.85 \mathrm{E}-05$ \\
\hline
\end{tabular}

ND: Relative abundance was below detectable limits by $16 \mathrm{~S}$ sequencing

NA: Statistical comparison not applicable due to a relative abundance below detectable limits (ND) in at least one GM group 
Supplementary table 4. Significantly different ileal scrape OTUs between GMJAX and GMHSD

\begin{tabular}{|c|c|c|c|c|c|}
\hline \multicolumn{2}{|c|}{ Significant OTUs in B6- $A p c^{+/ M i n}(p<0.001)$} & \multicolumn{2}{|c|}{$\begin{array}{c}\text { GMHSD } \\
\text { relative to GMJAX }\end{array}$} & \multirow{2}{*}{$\begin{array}{c}\text { GMJAX } \\
\text { Mean Relative } \\
\text { Abundance }( \pm \text { SEM) }\end{array}$} & \multirow{2}{*}{$\begin{array}{c}\text { GMHSD } \\
\text { Mean Relative } \\
\text { Abundance }( \pm \text { SEM })\end{array}$} \\
\hline Phylum & OTU & $p$-value & $\begin{array}{c}\text { Mean } \\
\text { Fold } \\
\text { Change }\end{array}$ & & \\
\hline Proteobacteria & Genus Parasutterella & $4.56 \mathrm{E}-31$ & 481.21 & $9.63 \mathrm{E}-06 \pm 6.99 \mathrm{E}-06$ & $0.0101 \pm 0.0012$ \\
\hline Proteobacteria & Genus Desulfovibrio & $1.64 \mathrm{E}-15$ & 201.56 & $5.72 \mathrm{E}-05 \pm 5.72 \mathrm{E}-05$ & $0.0140 \pm 0.0028$ \\
\hline Bacteroidetes & Family Muribaculaceae & $4.02 \mathrm{E}-15$ & 47.486 & $0.0001 \pm 5.64 \mathrm{E}-05$ & $0.0072 \pm 0.0010$ \\
\hline Firmicutes & Genus Butyricicoccus & NA & NA & ND & $0.0009 \pm 0.0003$ \\
\hline Firmicutes & Family Peptostreptococcaceae & $5.92 \mathrm{E}-10$ & 0.0368 & $0.0148 \pm 0.0042$ & $0.0005 \pm 0.0002$ \\
\hline Cyanobacteria & Order Gastranaerophilales.1 & NA & NA & ND & $0.0031 \pm 0.0013$ \\
\hline Proteobacteria & Genus Bilophila & NA & NA & ND & $0.0014 \pm 0.0003$ \\
\hline Firmicutes & Genus Lachnospiraceae UCG-001 & NA & NA & ND & $0.0017 \pm 0.0009$ \\
\hline Firmicutes & Genus Ruminococcaceae UCG-014.1 & 2.34E-09 & 37.965 & $0.0003 \pm 0.0001$ & $0.0113 \pm 0.0029$ \\
\hline Bacteroidetes & Genus Muribaculum & $1.90 \mathrm{E}-08$ & 0.41829 & $0.0565 \pm 0.0048$ & $0.0236 \pm 0.0024$ \\
\hline Bacteroidetes & Genus Alloprevotella.2 & $1.32 \mathrm{E}-07$ & 22.386 & $5.95 \mathrm{E}-05 \pm 5.32 \mathrm{E}-05$ & $0.0016 \pm 0.0004$ \\
\hline Bacteroidetes & Genus Rikenella & NA & NA & ND & $0.0007 \pm 0.0002$ \\
\hline Firmicutes & Genus Ruminococcaceae UCG-014 & $6.87 \mathrm{E}-07$ & 13.226 & $0.0001 \pm 4.89 \mathrm{E}-05$ & $0.0015 \pm 0.00041$ \\
\hline Actinobacteria & Genus Bifidobacterium & $1.19 \mathrm{E}-06$ & 29.214 & $8.98 \mathrm{E}-05 \pm 5.08 \mathrm{E}-05$ & $0.0029 \pm 0.0008$ \\
\hline Bacteroidetes & Genus Alistipes. 1 & 2.19E-06 & 7.0451 & $0.0008 \pm 0.00054$ & $0.0054 \pm 0.0019$ \\
\hline Verrucomicrobia & Genus Akkermansia & $2.71 \mathrm{E}-06$ & 27.871 & $0.0011 \pm 0.0003$ & $0.0301 \pm 0.0064$ \\
\hline
\end{tabular}




\begin{tabular}{|c|c|c|c|c|c|}
\hline Tenericutes & Genus Anaeroplasma & $6.98 \mathrm{E}-06$ & 0.12163 & $2.13 \mathrm{E}-05 \pm 1.49 \mathrm{E}-05$ & $0.0118 \pm 0.0093$ \\
\hline Proteobacteria & Genus Parasutterella.1 & $1.50 \mathrm{E}-05$ & 0.22455 & $0.0218 \pm 0.0035$ & $0.0049 \pm 0.0011$ \\
\hline Firmicutes & Genus Eubacterium oxidoreducens & $2.62 \mathrm{E}-05$ & 0.07639 & $0.0054 \pm 0.0011$ & $0.0004 \pm 0.0001$ \\
\hline Firmicutes & Genus Dubosiella & $3.69 \mathrm{E}-05$ & 58.775 & $6.35 \mathrm{E}-06 \pm 6.35 \mathrm{E}-06$ & $0.0011 \pm 0.0003$ \\
\hline Deferribacteres & Genus Mucispirillum & $9.35 \mathrm{E}-05$ & 89.705 & $4.75 \mathrm{E}-05 \pm 2.62 \mathrm{E}-05$ & $0.0052 \pm 0.0026$ \\
\hline Firmicutes & Genus Roseburia.1 & NA & $\mathrm{NA}$ & ND & $0.0004 \pm 0.0001$ \\
\hline Firmicutes & Genus Ruminococcaceae UCG-003 & 0.000125 & 0.18607 & $0.0045 \pm 0.0008$ & $0.0008 \pm 0.0002$ \\
\hline Proteobacteria & Azospirillum sp.47_25 & NA & NA & ND & $0.0013 \pm 0.0006$ \\
\hline Firmicutes & Genus Coprococcus & 0.000789 & 145.18 & $8.54 \mathrm{E}-06 \pm 8.54 \mathrm{E}-06$ & $0.0030 \pm 0.0022$ \\
\hline Firmicutes & Genus Erysipelatoclostridium & NA & NA & ND & $0.0005 \pm 0.0002$ \\
\hline Firmicutes & Genus Roseburia & 0.000911 & 0.25049 & $0.0035 \pm 0.0012$ & $0.0009 \pm 0.0003$ \\
\hline
\end{tabular}

ND: Relative abundance was below detectable limits by $16 \mathrm{~S}$ sequencing

NA: Statistical comparison not applicable due to a relative abundance below detectable limits (ND) in at least one GM group 
Supplementary table 5. Summary of metabolic pathways modulated by genetic lineage and GM

\begin{tabular}{|l|l|l|l|}
\hline Pathway Modulated & $\begin{array}{l}\text { Modulating } \\
\text { factor }\end{array}$ & $\begin{array}{l}\boldsymbol{p} \text { value } \\
\text { (corrected) }\end{array}$ & $\begin{array}{l}\text { Overlapping } \\
\text { metabolites }\end{array}$ \\
\hline Sphingosine/sphingosine-1-phosphate metabolism & GM & $3.3 \times 10^{-6}$ & 3 \\
\hline Morphine biosynthesis & GM & $1.7 \times 10^{-5}$ & 7 \\
\hline Bile acid biosynthesis/metabolism & Genetic lineage & 0.1 & 4 \\
\hline
\end{tabular}


Supplementary table 6. Summary of heterozygous and homozygous private variants associated with $\mathrm{Min} / \mathrm{J}$ and $\mathrm{Min} / \mathrm{D}$ colonies

\begin{tabular}{|c|c|c|c|c|c|c|c|c|}
\hline \multirow{2}{*}{} & \multicolumn{2}{|c|}{ VEP 0 } & \multicolumn{2}{c|}{ VEP 1 } & \multicolumn{2}{c|}{ VEP 2 } & \multicolumn{2}{c|}{ Total } \\
\cline { 2 - 9 } & Het & Hom & Het & Hom & Het & Hom & Het & Hom \\
\hline Min/D & 14,996 & 475 & 3,917 & 109 & 54 & 0 & 18,967 & 584 \\
\hline Min/J & 18,467 & 388 & 4,772 & 115 & 94 & 1 & 23,333 & 504 \\
\hline
\end{tabular}


Supplementary table 7. Over-represented biological pathways and candidate genes identified in $\mathrm{Min} / \mathrm{J}$

\begin{tabular}{|l|l|l|l|}
\hline Pathway name & Database & $\begin{array}{l}\text { P value } \\
\text { (corrected) }\end{array}$ & Candidate genes \\
\hline Neurotransmitter Receptor Binding & REACTOME & $1.29 \times 10^{-2}$ & Dlg3, Gabrb1, Grial \\
\hline Ion channel transport & REACTOME & $1.53 \times 10^{-2}$ & Casq2, Gabrb1, Mcoln3 \\
\hline Neuronal System & REACTOME & $1.57 \times 10^{-2}$ & Dlg3, Gabrb1, Grial, Kcnb2 \\
\hline Transmission across Chemical Synapses & REACTOME & $1.75 \times 10^{-2}$ & Dlg3, Gabrb1, Grial \\
\hline Stimuli-sensing channels & REACTOME & $3.52 \times 10^{-2}$ & Casq2, Mcoln3 \\
\hline Small cell lung cancer & KEGG & $3.84 \times 10^{-2}$ & Fhit, Myc \\
\hline TGF-beta signaling pathway & KEGG & $4.21 \times 10^{-2}$ & Acvr1, Myc \\
\hline Ubiquitin mediated proteolysis & KEGG & $6.97 \times 10^{-2}$ & Herc2, Ube2e3 \\
\hline
\end{tabular}

Candidate genes are genes that are found in the over-represented pathways and contained SNPs or indels private to the

Min/J line. Corrected $p$ values were calculated using the Benjamini-Hochberg method 
Supplementary table 8. Over-represented biological pathways and candidate genes identified in Min/D

\begin{tabular}{|l|l|l|l|}
\hline Pathway name & Database & P value (corrected) & Candidate genes \\
\hline Bile acid and bile salt metabolism & Reactome & $8.16 \times 10^{-2}$ & Cyp39al, Fabp6 \\
\hline Platelet aggregation & Reactome & $8.76 \times 10^{-2}$ & Rapgef4, Syk \\
\hline Integration of energy metabolism & Reactome & $9.28 \times 10^{-2}$ & Cacnala, Cacnalc, Rapgef4 \\
\hline
\end{tabular}

Candidate genes defined as those found in over-represented pathways and contained SNPs or indels private to the Min/D line.

Corrected $p$ values were calculated using the Benjamini-Hochberg method. 
Supplementary table 9. RT-qPCR primer/probe sets for mouse Fabp6, Cyp39a1, Myc, Dlg3, and Hprt

\begin{tabular}{|c|c|c|c|}
\hline Gene (IDT assay no.) & Probe & Primer 1 & Primer 2 \\
\hline $\begin{array}{c}\text { Fabp6 } \\
\text { (Mm.PT.58.41459866) }\end{array}$ & $\begin{array}{c}5^{\prime}-/ 56- \\
\text { FAM/CTCCACCAA/ZEN/C } \\
\text { TTGTCACCCACGA/31ABk } \\
\text { FQ/-3' }\end{array}$ & $\begin{array}{c}\text { CCCAACTATCACCAGA } \\
\text { CTTCG }\end{array}$ & GCCAGCCTCTTGCTTACG \\
\hline $\begin{array}{c}\text { Cyp39a1 } \\
\text { (Mm.PT.58.28626812) }\end{array}$ & $\begin{array}{c}\text { 5'-/56- } \\
\text { FAM/TGCTATGGG/ZEN/A } \\
\text { AACCGAATGACCTTTGT/ } \\
\text { 31ABkFQ/-3' }\end{array}$ & $\begin{array}{c}\text { GATTGGAGCTGGACTT } \\
\text { GAGTT }\end{array}$ & $\begin{array}{c}\text { GAAGCACATTGATTCCTTC } \\
\text { TTCT }\end{array}$ \\
\hline Hprt (Mm.PT.39a.22214828) & $\begin{array}{c}5^{\prime}- \\
\text { /5HEX/CTTGCTGGT/ZEN/ } \\
\text { GAAAAGGACCTCTCGAA } \\
\text { /31ABkFQ/-3' }\end{array}$ & $\begin{array}{c}\text { AACAAAGTCTGGCCTG } \\
\text { TATCC }\end{array}$ & $\begin{array}{c}\text { CCCCAAAATGGTTAAGGTT } \\
\text { GC }\end{array}$ \\
\hline Myc (Mm.PT.58.28494642) & $\begin{array}{c}\text { 5'-/56- } \\
\text { FAM/CGGTGTCTC/ZEN/C } \\
\text { TCATGCAGCACT/31ABkF } \\
\text { Q/-3' }\end{array}$ & $\begin{array}{c}\text { TTCTCTCCTTCCTCGGA } \\
\text { CTC }\end{array}$ & $\begin{array}{c}\text { CTTCCTCATCTTCTTGCTCT } \\
\text { TCT }\end{array}$ \\
\hline Dlg3 (Mm.PT.58.42693890) & $\begin{array}{c}5^{\prime}-/ 56- \\
\text { FAM/AGAGAAACT/ZEN/ } \\
\text { GAGTCCCTGGCTTGG/31 } \\
\text { ABkFQ/-3' }\end{array}$ & $\begin{array}{c}\text { GTGAGAAGAGGTCCTT } \\
\text { GTATGTC }\end{array}$ & $\begin{array}{c}\text { CGTTAATGACGTGCAGAA } \\
\text { TGTC }\end{array}$ \\
\hline
\end{tabular}




\section{CHAPTER III}

Bilophila wadsworthia Supplementation Modulates the Gut Microbiota, Metabolome, and Decreased Tumor Burden in Min Mice

(Jacob Moskowitz, Zhentian Lei, Lloyd W. Sumner, and

James Amos-Landgraf) 


\subsection{Overview}

Colorectal cancer (CRC) is a multifactorial disease comprising both genetic and environmental etiologies (Lichtenstein, Holm et al. 2000, de la Chapelle 2004). The gut microbiota (GM) is a dynamic collection of microbial species shaped by environmental factors such as geographical location and diet, and has emerged as a critically important component of mammalian physiology (Brim, Yooseph et al. 2013, Dejea, Wick et al. 2014). In recent years, the development of culture-independent methods of bacterial identification such as $16 \mathrm{~S}$ rRNA sequencing has revolutionized our capacity to understand the complex ecological community defined as the GM. With the aid of such technologies, studies have shown that changes in the GM are associated with a multitude of disease processes including CRC, suggesting that some bacterial species may contribute to pathogenesis through mechanisms such as genotoxin production, inflammation, and metabolic changes (Travaglione, Fabbri et al. 2008, Cuevas-Ramos, Petit et al. 2010, Carbonero, Benefiel et al. 2012, Couturier-Maillard, Secher et al. 2013). As such, elucidating protective or deleterious roles of specific taxa will improve risk assessment, prevention, and therapy for CRC.

Bilophila wadsworthia is a gram-negative, relatively low-abundance species that utilizes sulfites from taurine-conjugated bile acids as an electron receptor, ultimately producing hydrogen sulfide $\left(\mathrm{H}_{2} \mathrm{~S}\right)$ as a byproduct. This particular species expands after exposure to high-fat content that induces bile acid release into the intestines, and can regulate both inflammation and metabolic pathways (Natividad, Lamas et al. 2018). Though $B$. wadsworthia is regularly found in healthy fecal samples, it is frequently recovered from clinical appendicitis isolates (Baron, Summanen et al. 1989). However, 
its role as an instigator or bystander in these infectious cases is unclear. Taken together, B. wadsworthia is often considered an opportunistic pathogen that may contribute to the prevalence of obesity and metabolic syndrome associated with western diets.

While $B$. wadsworthia's immunogenicity and metabolic influence have been well described, its role as an etiologic, protective, or bystanding agent in CRC is currently unknown. One study reported enriched B. wadsworthia in a CRC cohort, but these associations have been inconsistent across different populations (Yazici, Wolf et al. 2017). To the authors' knowledge, there are no studies addressing the causative role of $B$. wadsworthia in adenoma initiation and development, nor do we understand how this species influences the broader GM and metabolism in the context of CRC. Given our growing understanding of the physiological relevance of $B$. wadsworthia, there is a significant need to explore how this species influences cancer initiation and progression.

The challenges of controlling for genetic and environmental variability, and limited ability to conduct prospective studies in humans, emphasize the importance of preclinical cancer models to establish causal relationships. The C57BL/6J-Apc ${ }^{\text {in }}\left(A p c^{M i n}\right)$ mouse has a dominant mutation in the Apc gene resulting in the development of intestinal adenomas. This model provides a quantifiable phenotype recapitulating early carcinogenesis, and serves as a platform to test genetic or environmental modifiers of disease (Shoemaker, Gould et al. 1997). Here, we use a combination of specificpathogen-free (SPF) $A p c^{M i n}$ mice, microbial $16 \mathrm{~S}$ rRNA analysis, and targeted and untargeted metabolomics to explore how B. wadsworthia contributes to adenoma initiation and development, and its capacity to modulate the GM and host metabolism. We hypothesized that addition of $B$. wadsworthia to an existing complex GM would 
result in increased adenoma burden. Here, we show that $B$. wadsworthia supplementation unexpectedly suppressed both adenoma multiplicity and overall adenoma burden, and it further influenced the complex GM and host metabolism. Together, these results suggest that $B$. wadsworthia may inhibit initiation and progression of adenomas, and could have a positive role in CRC prevention.

\subsection{Methods}

\subsubsection{Ethics Statement}

Animal studies were conducted in an Association for Assessment and Accreditation of Laboratory Animal Care International (AAALAC) accredited facility based on the guidelines provided by the Guide for the Care and Use of Laboratory Animals. All animal studies were approved by the University of Missouri Institutional Animal Care and Use Committee.

\subsubsection{Animals}

All mice were purchased from The Jackson Laboratory (Bar Harbor, ME), and acclimated for several generations prior to use. Six to eight week old male C57BL/6J$A p c^{\mathrm{Min}} /+$ mice were each set up in mating trios with two wild-type six to eight week old female C57BL/6J mice. After females were confirmed pregnant, they were individually housed and assigned to one of three experimental groups: control (PBS), B. wadsworthia, or B. wadsworthia + taurine. Pregnant dams in the 'B. wadsworthia + taurine' group were given $1 \%$ taurine (Sigma-aldrich, St. Louis, MO) in drinking water until their pups were weaned. At weaning (21 days of age), offspring were group-housed with sex-matched 
littermates within their experimental group. To genotype for the Min allele, all offspring were ear-punched at weaning. DNA was extracted using the "HotSHOT" genomic DNA preparation method as described (Truett, Heeger et al. 2000). Fecal samples were collected from all Min offspring at weaning and 40 days of age (D40), and stored at -80 ${ }^{\circ} \mathrm{C}$. All experimental groups were housed in micro-isolator cages on ventilated racks (Thoren, Hazelton, PA). All mice had ad libitum access to 5058 irradiated breeder chow (LabDiet, St. Louis, MO) and acidified autoclaved water, and were maintained on paper chip bedding (Shepherd Specialty Papers, Watertown, TN) with a 14:10 light:dark hour cycle.

\subsubsection{Bilophila wadsworthia culture and treatment}

Bilophila wadsworthia was purchased from American Type Culture Collection (ATCC \#49260; Manassas, VA), grown in PYG-BILE media (Anaerobe Systems, Morgan Hill, CA), then flash-frozen in glycerol solution (25\% v/v glycerol) and stored at $-80{ }^{\circ} \mathrm{C}$. For treatment, $1 \mathrm{~mL}$ of frozen stock was thawed, inoculated into $10 \mathrm{~mL}$ PYGBILE medium, and incubated at $37^{\circ} \mathrm{C}$. After reaching the late log-phase of growth based on optical density of pure culture, bacteria was pelleted by centrifugation and washed twice with 1x phosphate-buffered saline (PBS) pH 7.3 (Ausubel FM 1994). The pellet was resuspended in PBS to a final concentration of 5.0x $10^{7}-5.0 \times 10^{8} \mathrm{CFUs} / 250 \mu \mathrm{L}$ PBS. Intra-gastric gavage was used to treat pregnant dams with either $250 \mu \mathrm{L} \mathrm{PBS}$ (controls), or $250 \mu \mathrm{L}$ PBS containing 5.0x $10^{7}-5.0 \times 10^{8}$ CFUs B. wadsworthia (treatment groups) on days 15 and 18 of pregnancy, and days 2 and 5 after pups were delivered. All Min offspring were themselves administered one additional treatment at 25 days of age (Fig. 1A). 


\subsubsection{Bilophila wadsworthia colonization}

Fecal samples collected from Min offspring at weaning, D40, and 3 months, and ileal epithelial scrapes (see supplementary methods) were used to test colonization levels of $B$. wadsworthia by quantitative PCR (qPCR) analysis. All B. wadsworthia quantification is expressed as copy number relative to $16 \mathrm{~S}$ eubacterial copy number (see table S4 for all primer/probe sequences). For fecal samples, each $10 \mu \mathrm{L}$ reaction contained $5 \mu \mathrm{L}$ of $2 \mathrm{x}_{\text {iTaq }}{ }^{\mathrm{TM}}$ Universal SYBR ${ }^{\circledR}$ Green Supermix (Bio-Rad, Hercules, $\mathrm{CA}$ ), $2 \mu \mathrm{L}$ of $2 \mu \mathrm{M}$ forward and reverse primers (final concentration $400 \mathrm{nM}$ ), and $1 \mathrm{ng}$ DNA template. PCR parameters were: initial incubation at $95^{\circ} \mathrm{C}$ for 10 minutes, denaturation at $95^{\circ} \mathrm{C}$ for 15 seconds, annealing and elongation at $60{ }^{\circ} \mathrm{C}$ for 1 minute for a total of 45 cycles. For ileal epithelium, a B. wadsworthia primer/probe mix was designed due to non-specific amplification with the aforementioned assay (table S4). Each $10 \mu \mathrm{L}$ reaction contained $0.5 \mu \mathrm{L}$ of $20 \mathrm{x}$ primer/probe mix (IDT®), $5 \mu \mathrm{L}$ of $2 \mathrm{x}^{\text {iTaq }}{ }^{\mathrm{TM}}$ Universal Probe Supermix, 3.5 $\mu \mathrm{L}$ nuclease-free water, and 20-100 ng DNA template. PCR parameters were: denaturation at $95{ }^{\circ} \mathrm{C}$ for 5 seconds, and annealing and elongation at 60 ${ }^{\circ} \mathrm{C}$ for 30 seconds for a total of 54 cycles. All reactions were performed in triplicate. Standard curves were generated using DNA extracted from pure $B$. wadsworthia culture using both $B$. wadsworthia primers and 16S V4 eubacterial primers, and were subsequently used to estimate copy numbers in fecal and epithelial samples.

\subsubsection{Adenoma multiplicity and burden}

At 3 months of age all Min mice were euthanized via $\mathrm{CO}_{2}$ asphyxiation. Whole small and large intestines were removed from the abdominal cavity. The small intestines were cut into four equal lengths (S1-S4). All sections including the colon were opened 
longitudinally, flushed with saline and formalin-fixed on bibulous paper with the luminal side facing up. Grossly visible adenomas were counted manually using a Leica M165FC microscope (Leica, Buffalo Grove, IL) at 1.25x magnification. Adenoma sizes were measured using the Leica Application Suite 4.2, and were used for adenoma burden calculations. The diameter of all adenomas in an individual were measured, and then used to calculate estimated spherical volume of each adenoma using the formula $\left(v=4 / 3 \pi r^{3}\right)$

(Crist, Roth et al. 2010). Estimated volume of all SI and colon adenomas were summed to determine overall SI and colon adenoma burdens, respectively.

\subsubsection{Tissue and sample collection and processing}

Mice were humanely euthanized via $\mathrm{CO}_{2}$ asphyxiation at 3 months of age followed by necropsy for tissue and sample harvesting. A sterile scalpel blade was used to scrape a section of normal ileal epithelium, and fecal samples were collected from the distal colon. Intra-cardiac extraction of blood was performed immediately following euthanasia, and serum was separated by centrifugation at $2000 \mathrm{~g}$ for 10 minutes. All samples were flash-frozen in liquid nitrogen followed by storage at $-80{ }^{\circ} \mathrm{C}$.

\subsubsection{DNA extraction for quantitative PCR and 16S rRNA sequencing}

All samples were mechanically disrupted using a TissueLyser II (Qiagen, Venlo, Netherlands) for 2 minutes at $50 \mathrm{~Hz}$, then incubated at $70{ }^{\circ} \mathrm{C}$ for 20 minutes with periodic vortexing. DNA extraction from fecal pellets for $16 \mathrm{~S}$ rRNA sequencing was performed using a DNeasy Blood \& Tissue Kit® (Qiagen) as previously described (Ericsson, Davis et al. 2015). DNA extraction from ileal epithelium scrapes were performed using the AllPrep ${ }^{\circledR}$ DNA/RNA Mini Kit (Qiagen) according to the manufacturer's instructions. 


\subsubsection{S library preparation, sequencing, and informatics processing}

DNA was extracted from feces and ileal epithelium as described (see supplementary methods) and processed at the University of Missouri DNA Core (Columbia, MO). Primers (U515F/806R) were used to generate an amplicon library of the hypervariable V4 region of the bacterial $16 \mathrm{~S}$ rRNA gene as previously reported (Caporaso, Lauber et al. 2010). Amplicons were sequenced on the Illumina MiSeq platform under previously described conditions (Montonye, Ericsson et al. 2018). Trimming, assembly, binning, and annotation of DNA sequences was performed at the University of Missouri Informatics Research Core Facility (Columbia, MO). Quality control of DNA, assembly of contiguous sequences, sequence removal after trimming for base quality, and chimera removal were completed (Montonye, Ericsson et al. 2018). Sequences were assigned to operational taxonomic units (OTUs) by de novo OTU clustering based on $97 \%$ nucleotide similarity. OTUs were annotated by referencing BLAST (Altschul, Madden et al. 1997) against the SILVA database (Ritari, Salojarvi et al. 2015) of 16s rRNA phylogeny.

\subsubsection{Ultra-high Performance Liquid Chromatography-Mass Spectrometry (UHPLC-MS)}

Samples from the control and B. wadsworthia + taurine groups were used for UHPLC-MS analysis. Fecal and SI content samples were frozen and then lyophilized at $20{ }^{\circ} \mathrm{C}$ using 0.1 millibar of vacuum pressure. Dried samples were weighed and suspended to a final concentration of $10 \mathrm{mg}$ dried sample $/ \mathrm{mL} 80 \% \mathrm{MeOH}+18 \mu \mathrm{g} / \mathrm{mL}$ umbelliferone as an internal standard. For serum samples, $50 \mu \mathrm{L}$ of serum was suspended in $450 \mu \mathrm{L} 100 \% \mathrm{MeOH}$ with $18 \mu \mathrm{g} / \mathrm{mL}$ umbelliferone and $0.1 \%$ formic acid. Samples 
were sonicated for 5 minutes and centrifuged for 40 minutes at $3000 \mathrm{~g}$, then a $100 \mu \mathrm{l}$ aliquot was transferred to microvolume inserts. For UHPLC-MS analysis, a Bruker maXis impact quadrupole-time-of-flight mass spectrometer coupled to a Waters ACQUITY UPLC system was used. Extracted compounds were separated with a Waters BEH C18 column (2.1x 150 mm with 1.7-um particles) using a linear gradient and mobile phase A (0.1\% formic acid in water) and B (100\% acetonitrile). The initial mobile phase composition was $95 \% \mathrm{~A}$ and $5 \% \mathrm{~B}$. Phase B was linerarly increased from $5 \%$ to $70 \% \mathrm{~B}$ from 0 to $30 \mathrm{~min}$, then to $95 \% \mathrm{~B}$ over $3 \mathrm{~min}$, held at $95 \% \mathrm{~B}$ for $3 \mathrm{~min}$, then returned to $5 \% \mathrm{~B}$ for equilibration. The flow rate was $0.56 \mathrm{~mL} / \mathrm{min}$ and the column temperature was $60^{\circ} \mathrm{C}$. Mass spectrometry was performed in the negative electrospray ionization mode with the nebulization gas pressure at 43.5 psi, dry gas of $121 / \mathrm{min}$, dry temperature of $250{ }^{\circ} \mathrm{C}$ and a capillary voltage of $4000 \mathrm{~V}$. Mass spectral data were autocalibrated using sodium formate after data acquisition. For targeted bile acid analyses, purified standard compounds were purchased from Sigma-Aldrich (DCA, CDCA, LCA, CA, UDCA, TCA) and Steraloids, Inc. (Newport, RI) (MCA $\alpha$, TMCA $\alpha$, MCA $\beta$, TMCA $\beta$ ). Standards were each suspended in $\mathrm{MeOH}$ to a final concentration of 500 $\mu \mathrm{g} / \mathrm{mL}$, then analyzed under the same conditions described above.

\subsubsection{Statistical analysis}

Statistical analyses of B. wadsworthia qPCR data and 16S rRNA data were performed using Sigma Plot 14.0 (Systat Software Inc., Carlsbad CA). To generate a list of all significantly modulated $(\mathrm{q}<0.05)$ OTUs, relative abundance data was entered into the Metaboanalyst v3.0 web program (Xia, Sinelnikov et al. 2015), and ANOVA with Tukey's post-hoc test was performed. PCA biplots were generated using the 
Paleontological Statistics software package (PAST) 3.12 (Hammer, Harper et al. 2001). PCA were performed using an eigenvalues of the variance-covariance matrix and plotted on the eigenvalue scale using 1/4-root transformed data. Statistical testing of adenoma counts and burden were performed using Analysis of Variance (ANOVA) with the Student Newman-Keul post-hoc test in Sigma Plot 14.0, where $p<0.05$ was considered significant. GraphPad Prism 8 was used to generate all scatter plots, bar graphs, and Pearson's correlations.

\subsection{Results}

\subsubsection{Bilophila wadsworthia modulates the gut microbiota}

To determine the effects of Bilophila wadsworthia on the gut microbiota, metabolism, and adenoma susceptibility in $A p c^{M i n}$ mice, pregnant dams were assigned to one of three groups: controls, B. wadsworthia, or B. wadsworthia + taurine. The additional group receiving supplemental $1 \%$ taurine in drinking water was included to aid with successful B. wadsworthia colonization, due to the bacteria's primary use of taurine as a source of sulfite. Pregnant dams were treated with PBS (control group) or 5.0x10 5.0x $10^{8}$ CFUs B. wadsworthia (treatment groups) prior to, and after pups were delivered to ensure that $A p c^{M i n}$ offspring were colonized with $B$. wadsworthia immediately upon birth (Fig. 1A). Apc $c^{\mathrm{Min}}$ offspring also received an additional PBS or bacteria treatment after weaning (day 25). Quantitative PCR analysis of $A p c^{\text {Min }}$ fecal samples at weaning, day 40, 3 months, and ileal epithelium scrapes revealed significantly increased relative abundance of B. wadsworthia in both treatment groups relative to controls (Fig. 1B). 
Notably, there was no appreciable difference in colonization between the $B$. wadsworthia and B. wadsworthia + taurine treatment groups.

Fecal and ileal epithelium samples were also used to determine how the addition of $B$. wadsworthia influenced the broader GM community using $16 \mathrm{~S}$ rRNA sequencing. At the phylum level, we did not note any changes in the Firmicutes:Bacteroidetes ratio, but there was a substantial increase in the relative abundance of Proteobacteria in treated mice (Fig. 1C). As the Proteobacteria phylum was exclusively comprised of $B$. wadsworthia, our B. wadsworthia supplementation accounts for the enrichment of this phylum. Interestingly, we observed that Prevotella sp. in feces and ileal epithelium were largely abolished in both groups treated with B. wadsworthia (Fig. 1C, supplementary table 1), and this loss persisted throughout the duration of the study until the 3 month endpoint. Principal Component Analysis with overlaid loadings plots (biplot) were used to visualize overall GM composition of control and treated groups and to identify OTUs primarily driving the differences in $\beta$-diversity between groups. Biplots representing GM communities in feces at day 40 and 3 months, and in the ileal epithelium, demonstrate distinct clustering of controls compared to treatment groups (Fig. 1D). Enrichment of Bilophila sp. was strongly associated with the treated groups in all analyzed samples, while enriched Prevotella sp. was associated with controls. In ileal epithelium, Prevotella sp. and an OTU from the family Muribaculacea were enriched in controls while there was increased Lachnospiraceae in treated mice. Thus, the addition of B. wadsworthia to a complex community previously lacking this microbe influenced relative abundance of various taxa, but distinctly resulted in long-term loss of Prevotella sp.

\subsubsection{B. wadsworthia supplementation suppresses SI adenomas in Min mice}


Thus far, we have demonstrated that the GM changes following the introduction of $B$. wadsworthia. Additionally, previous studies have demonstrated metabolic and inflammatory changes associated with B. wadsworthia. (Devkota, Wang et al. 2012, Natividad, Lamas et al. 2018). Thus, we explored whether these physiological changes associated with $B$. wadsworthia affect adenoma initiation and progression in genetically susceptible $A p c^{M i n}$ mice. At 3 months of age, control and treated mice were humanely sacrificed for adenoma phenotyping. After manual counting of SI and colonic adenomas, we observed significantly reduced SI adenoma multiplicity in both B. wadsworthia treatment groups compared to controls (Fig. 2A). All observed adenomas were also measured by diameter (Fig. S1), which was subsequently used to estimate adenoma volume (see materials and methods). For each individual, all estimated SI and colon adenoma volumes were summed to determine overall adenoma SI and colon adenoma burden, respectively. We noted reduced SI adenoma burden in B. wadsworthia-treated groups, while there were no significant differences noted in colon adenoma burden (Fig. 2B). Furthermore, we found that there was a negative correlation between fecal $B$. wadsworthia relative abundance and adenoma multiplicity across the two treated groups (controls were omitted from this analysis) (Fig. 2C). We also evaluated whether there was a positive correlation between adenoma multiplicity and Prevotella sp in controls and treated mice. In controls (Fig. 2D), we observed a positive correlative trend between Prevotella sp. in the ileal epithelium and adenoma number. The majority of treated animals had very low or undetectable levels of Prevotella. Interestingly though, three treated mice retained their Prevotella populations, two of which developed higher adenoma counts compared to the remainder of the treated cohort (Fig. 2E). To 
summarize, addition of $B$. wadsworthia to an existing complex GM results in reduced SI adenoma multiplicity, size, and overall burden, and colonization level specifically correlates with adenoma numbers among treated mice.

\subsubsection{B. wadsworthia modulates the fecal and serum metabolome}

We employed UHPLC-MS analyses of feces and serum to interrogate local and systemic changes in metabolism resulting from addition of $B$. wadsworthia. For these analyses, fecal, SI content, and serum samples from control and B. wadsworthia + taurine mice following sacrifice at 3 months were prepared. Taurine was removed from the water of pregnant dams when $A p c^{\text {Min }}$ pups were weaned. Therefore, we do not anticipate that taurine supplementation affected metabolite profiles at the terminal 3-month time-point. We used PCA analysis to visualize distinct untargeted metabolite profiles in feces (Fig. 3A), and relatively unaffected metabolite profiles in SI contents (Fig. S2). Differential metabolites were used to determine the dysregulation of KEGG pathways (Fig. 3A) in order to provide insight into broader biological pathways influenced by $B$. wadsworthia supplementation. Notably, bile acid (BA) biosynthesis was the most significantly dysregulated pathway, with seven out of 18 metabolites associated with BA biosynthesis being differentially accumulated in feces. PCA visualization also showed the discrete nature of serum metabolites in treated mice (Fig. 3B). Using a False Discovery Rate (qvalue) of 0.05 as a threshold, we found that 658 features were significantly differential in serum, with putative metabolite METLIN identifications given when applicable (supplementary table 2). Pathway analysis of serum metabolites further identified dysregulation of 4-hydroxy-2-nonenal detoxification in treated mice. Thus, untargeted metabolomics analysis demonstrates that $B$. wadsworthia directly or indirectly influences 
local and systemic metabolite profiles, and results in altered regulation of various biological pathways.

\subsubsection{B. wadsworthia supplementation modulates fecal and serum bile acid composition}

B. wadsworthia utilizes sulfite groups present in taurine as an energy source and can therefore affect taurine metabolism (Natividad, Lamas et al. 2018). Taurine is further involved in BA conjugation, and enrichment of B. wadsworthia may also alter BA metabolism, and thereby exert changes in BA-mediated signaling and carcinogenesis (Thomas, Pellicciari et al. 2008, Cao, Luo et al. 2014). Observations in the dysregulation of BA biosynthesis in treated mice, as determined by untargeted metabolomics, led us to develop a targeted metabolite analysis method to better understand how B. wadsworthia influences primary and secondary bile acids composition both locally and systemically. We used a panel of commercial authentic purified known primary and secondary bile acids to develop a targeted UHPLC-MS analysis method and then used this method for further studies. We observed increased relative levels of muricholic acid- $\beta$ (MCA $\beta$ ) and deoxycholic acid (DCA) in the feces of B. wadsworthia-treated mice compared to controls (Fig. 4A), while there were no detected differences between groups in SI contents (Fig. S3). In serum samples, there was a significant reduction in relative DCA levels after B. wadsworthia treatment (Fig. 4B). Thus, addition of Bilophila wadsworthia supplementation affects both primary and secondary bile acid composition. Taken together with our untargeted metabolomics demonstrating changes in BA biosynthesis, $B$. wadsworthia has a broad capacity to influence BA metabolism. 


\subsubsection{Untargeted metabolites correlate with $B$. wadsworthia and adenoma multiplicity}

Using PCAs to visualize untargeted fecal and serum metabolite profiles, we have established that $B$. wadsworthia supplementation affects metabolism both locally in the intestinal lumen, and systemically in the serum (Fig. 3). To follow up on these findings, we asked whether these changes could be used as a platform to identify candidate metabolites that are regulated directly or indirectly by B. wadsworthia and also have a protective role in adenoma initiation. To that end, we screened both fecal and serum untargeted metabolomics datasets for metabolites with the strongest correlations to adenoma multiplicity across control and B. wadsworthia-treated groups, of which several are shown (Fig. S4). These datasets were also screened for correlations with qPCRdetermined B. wadsworthia abundance in feces within the treated group (Fig. S5), while the control group was omitted from this analysis because there was no B. wadsworthia detected in control samples. To identify putative metabolites most likely to have a role in B. wadsworthia-mediated adenoma suppression, the aforementioned correlation data were cross-referenced to distinguish features with significant correlations to both $B$. wadsworthia relative abundance and adenoma multiplicity. We found 3 fecal metabolites, two of which were putatively identified as azalomycin and 1,3-diisopropylbenzene (Fig. 5A-B), and 3 serum metabolites (Fig. 5C-D) that fit such criteria. Of note, all candidate metabolites demonstrated a positive correlation with fecal B. wadsworthia abundance, and a negative correlation with adenoma multiplicity. In summary, using large-scale metabolomics datasets, we correlated individual features with two quantitative measures 
to determinediscriminate candidate metabolites mechanistically involved in $B$.

wadsworthia-mediated adenoma suppression.

\subsection{Discussion}

Numerous studies have demonstrated the gut microbiota's capacity to modulate CRC susceptibility (Ericsson, Akter et al. 2015, Chen and Vitetta 2018, Mendes, Paulino et al. 2018). The limitations of associative human studies necessitate the use of preclinical models to delineate how various microbial taxa influence the intestinal environment, and ultimately affect CRC initiation and progression. Using the $A p c^{M i n}$ mouse model, we herein describe the role of the resident human gut microbe, Bilophila wadsworthia, in CRC initiation and early progression in the context of APC-driven adenomas. Following the successful colonization of specific-pathogen-free $A p c^{M i n}$ mice with $B$. wadsworthia, we used a multi-omics approach to demonstrate $B$. wadsworthiamediated changes in the GM and metabolism, and further showed suppression of adenomas resulting from the introduction of this bacterium. Though these results proved our initial hypothesis incorrect, this work represents significant progress in our understanding of how $B$. wadsworthia modulates the intestinal environment in the context of CRC.

Presently, little is known regarding how B. wadsworthia affects broader GM communities. While studies have reported community changes associated with dietarymediated B. wadsworthia expansion, it is unclear how this organism influences the GM independent of the diet (Devkota, Wang et al. 2012, Natividad, Lamas et al. 2018). By sequencing the microbial 16S rRNA gene, we characterized changes in the GM attributed 
to $B$. wadsworthia supplementation. While there were no changes in the prominent Firmicutes and Bacteroidetes phyla, we observed a striking loss of the prevalent human genera Prevotella sp. in treated mice (Fig. 1C). Interestingly, the loss of Prevotella was not only seen in fecal samples, but also in the ileal epithelium of mice treated with $B$. wadsworthia. Enrichment of B. wadsworthia and concurrent loss of Prevotella in the ileal mucosa persisted at the 3 month experimental terminus despite cessation of supplementation at 25 days of age (Figs 1B-C). This suggests that these two organisms compete for a niche in the epithelium of the ileum, and factors such as high-fat diets that promote expansion of $B$. wadsworthia can simultaneously deplete Prevotella. Moreover, the close proximity of $B$. wadsworthia and Prevotella sp. to the epithelium further suggests that these bacteria have important roles in host-microbiota interactions. Bilophila wadsworthia utilizes sulfites in taurine-conjugated bile acids for anaerobic respiration, ultimately producing hydrogen sulfide $\left(\mathrm{H}_{2} \mathrm{~S}\right)$ as a byproduct (Ridlon, Wolf et al. 2016). Several studies have reported vastly different effects of $\mathrm{H}_{2} \mathrm{~S}$ in the context of CRC, with some suggesting a pro-carcinogenic genotoxic role, while others have demonstrated a protective role (Attene-Ramos, Wagner et al. 2006, AtteneRamos, Wagner et al. 2007, Chattopadhyay, Kodela et al. 2012). B. wadsworthia has become a very interesting species in the etiology of CRC due to its role in sulfur metabolism, and more recently its association with certain human CRC cohorts (Yazici, Wolf et al. 2017). Remarkably, we found that supplementing a complex SPF GM harbored by genetically susceptible $A p c^{M i n}$ mice resulted in both reduced adenoma multiplicity and overall adenoma burden (Figs. 2A-B). Given the aforementioned ablation of Prevotella sp. after supplementation, it is important to consider the potential 
for both direct and indirect effects mediated by B. wadsworthia. Of note, studies have described the immunogenic nature of $B$. wadsworthia characterized by induction of Th1 immune responses mediated by increased IL-12 and $\mathrm{CD} 4^{+} \mathrm{IFN} \gamma$, while there was no detectible Th17 response (Devkota, Wang et al. 2012). In contrast, Prevotella sp. distinctly induced Th17-mediated inflammation in vitro and in vivo via associated cytokines such as IL-17, IL-23, and IL-1 (Larsen 2017). Various studies have demonstrated the anti-tumorigenic role of Th1 immune responses, highlighted by a report of increased tumors in IFN $\gamma$ knockout mice exposed to azoxymethane and trinitrobenzene sulfonic acid relative to wild-type controls (Osawa, Nakajima et al. 2006). Moreover, increased Th1-mediated infiltrates are associated with a positive prognosis in human CRC outcome (Pages, Berger et al. 2005). Meanwhile, Th17 responses are positively associated with CRC, as higher levels of Th17 cytokines mark the early stages of pathogenesis (De Simone, Pallone et al. 2013). Furthermore, ablation of IL-17A in Apc ${ }^{\text {Min }}$ mice results in substantially reduced tumorigenesis, while enterotoxigenic Bacteroides fragilis (ETBF) enhances tumorigenesis in a Th17-dependent manner (Wu, Rhee et al. 2009, Chae, Gibson et al. 2010). As such, the loss of mucosal-associated Prevotella coinciding $B$. wadsworthia enrichment may dictate a relative shift from a Th17 to Th1 response, thereby conferring adenoma suppression in $A p c^{M i n}$ mice.

Targeted and untargeted metabolomics provide an avenue to interrogate metabolite profiles under controlled experimental settings, and can therefore provide insight into perturbed biological pathways in the context of disease. While an untargeted approach is highly sensitive and may reveal novel metabolites of interest, distinguishing true features from unidentified adducts and fragments is still a substantial challenge 
(Melnik, da Silva et al. 2017). Thus, using untargeted metabolomics in concert with a targeted approach to validate initial findings is an ideal strategy to glean valuable data from high-throughput techniques. Do such, we found that $B$. wadsworthia induced shifts in overall metabolite profiles of feces and serum (Fig. 3). This suggested that the introduction of $B$. wadsworthia to an established GM has a significant effect on metabolism, and may regulate specific metabolites responsible for $B$. wadsworthiamediated adenoma suppression (Fig. 5). To interrogate these changes further, we mapped putative differential metabolites to their associated KEGG pathways, and identified dysregulation of a number of pathways, the most striking of which was bile acid biosynthesis (Fig. 3A). Given that B. wadsworthia utilizes taurine from conjugated bile acids, it is not altogether surprising that this species regulates the enterohepatic axis. Moreover, previous studies have reported modulation of the bile acid composition after B. wadsworthia supplementation (Natividad, Lamas et al. 2018). However, it is unclear how bile acid metabolism is affected in the context of a genetically susceptible CRC model.

To expand upon our initial untargeted metabolomics analyses, we used a targeted approach to directly address how $B$. wadsworthia influences bile acid levels. Notably, we found significant modulation of deoxycholic acid (DCA), where DCA levels were higher in the feces, and lower in the serum of treated mice relative to controls (Figs 4A and B). DCA has been implicated in the pathogenesis of CRC through a number of different mechanisms including induction of reactive oxygen species, genomic destabilization, and resistance to apoptosis (Nguyen, Ung et al. 2018), though it can promote apoptosis when present in higher concentrations (Milovic, Teller et al. 2002). Furthermore, higher serum 
levels have been associated with increased CRC risk in human cohorts (Costarelli, Key et al. 2002), which is reflected in our observation of increased serum DCA in control mice (Fig. 4B). In this case, serum DCA levels did not reflect fecal levels, as fecal DCA was decreased in supplemented mice. This suggests that $B$. wadsworthia could modify recycling of secondary bile acids such that DCA is more readily excreted, accounting for its relative increase in feces and reduction in serum. Notably, a Prevotella-enriched GM has been associated with elevated plasma DCA levels, suggesting that diminished Prevotella after B. wadsworthia supplementation could account for reduced serum DCA (Gu, Wang et al. 2017). Presently, it is not clear how B. wadsworthia modulates potentially carcinogenic metabolites such as DCA, and further studies in controlled settings are required to elucidate these mechanisms.

As evidenced in this report, the precise role of B. wadsworthia is highly complex and dependent on the surrounding intestinal environment. Here, we establish that supplementing $B$. wadsworthia results in distinct changes in resident microbial taxa as shown by the first known interaction between Bilophila and Prevotella, and broadly modulates local and systemic metabolism. We show that these changes result in suppression of adenoma initiation and progression in $A p c^{M i n}$ mice, provide insight into potential mechanisms through which $B$. wadsworthia affects pathogenesis, and establish a platform to perform such studies in the future. Together, these data highlight the convoluted nature of host-microbe interactions in the context of CRC initiation and progression, and emphasize the need to incorporate preclinical studies using complex ecological systems alongside simplified gnotobiotic approaches to increase translatability. Finally, continued accumulation of data pertaining to the effects of 
microbial populations in CRC models will enhance our understanding of pre-existing risk factors, and ultimately drive the development of novel preventatives and therapeutics to reduce the prevalence of $\mathrm{CRC}$ in human populations.

\subsection{Author Contributions and Acknowledgements}

JM and JAL designed all experiments. JM performed all experiments. ZL and LS were instrumental in assisting with metabolite extractions, and performing metabolomics analyses. SB, KD, JW and JAL designed the experiments. The authors would like to acknowledge Nathan Bivens and the MU DNA Core for their role in 16S rDNA sequencing, and Bill Spollen, Christopher Bottoms and the MU Informatics Research Core Facility for assistance with 16S rRNA sequencing analysis.

\subsection{Financial Support}

This research was funded by grants from the National Institutes of Health to the Mutant Mouse Resource and Research Center at the University of Missouri (U42 OD010918), and by the University of Missouri to Dr. James Amos-Landgraf (Startupfunding). JM was supported by NIH T32 OD011126. The University of Missouri also provided support for the MU Metabolomics Center and the DNA Core Facility. 


\subsection{Figures}

Figure 1. Bilophila wasdworthia modulates the GM. A, Pregnant dams and 25 day old C57BL/6J-Apc ${ }^{\text {Min }}$ offspring were supplemented with PBS (controls; $\mathrm{n}=5$ ), 5.0x1075.0x $10^{8}$ CFUs B. wadsworthia $(\mathrm{n}=8)$, or $5.0 \times 10^{7}-5.0 \times 10^{8} \mathrm{CFUs} B$. wadsworthia with $1 \%$ taurine in drinking water $(n=7)$. B, Scatter plots comparing mean $( \pm S D)$ relative abundance of $B$. wadsworthia in feces across three time-points, and in ileal epithelum scrapes based on qPCR analysis. C, Bar graphs comparing mean $( \pm \mathrm{SD})$

Firmicutes:Bacteroidetes ratio, relative abundances of phylum Proteobacteria and Prevotella sp., determined by 16S rRNA sequencing. D, Principal Component Analysis (PCA) biplots visualizing differences in $\beta$-diversity at the OTU level between control and treatment groups. PCAs are overlaid with loadings plots, which identify OTUs primarily driving the differences in $\beta$-diversity. ${ }^{*} p<0.05,{ }^{* *} p<0.01,{ }^{* * *} p<0.001$; ANOVA with the Student Newman-Keuls method.

A

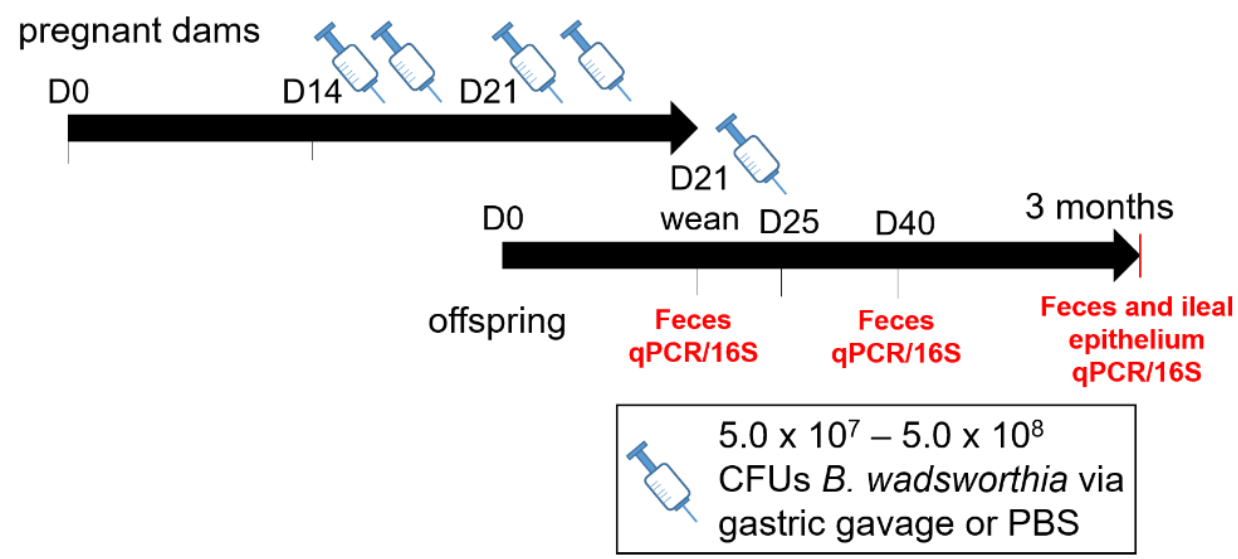


B

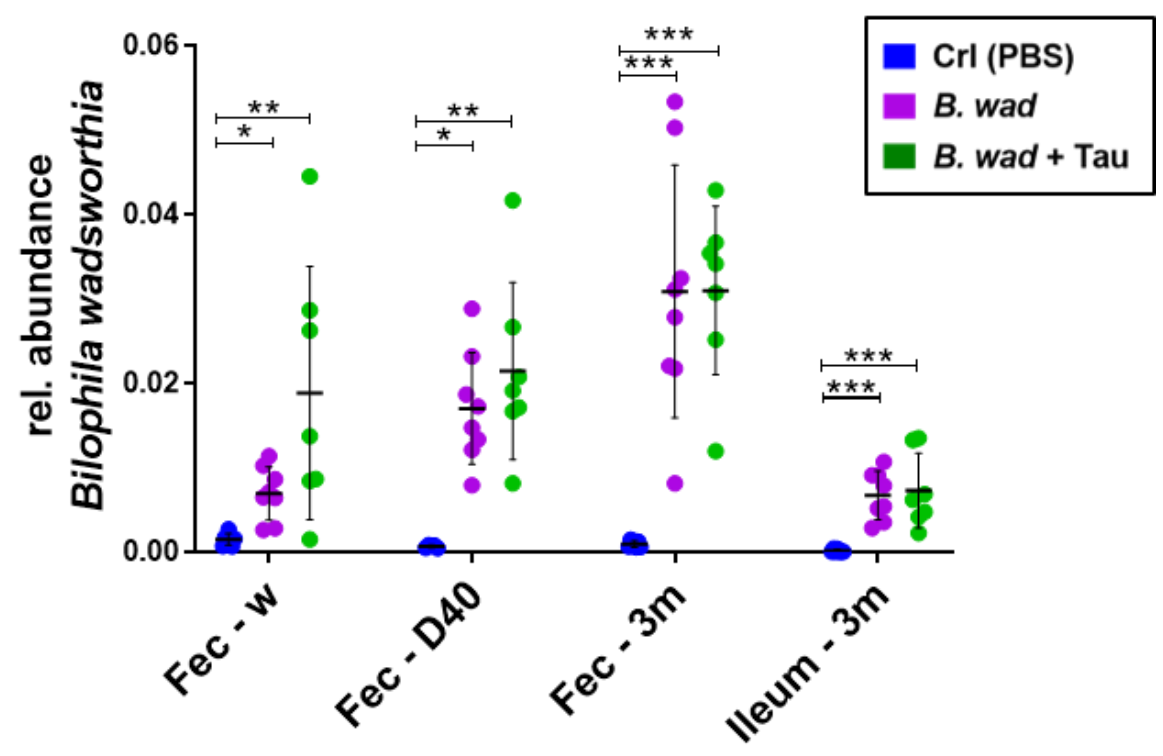

C
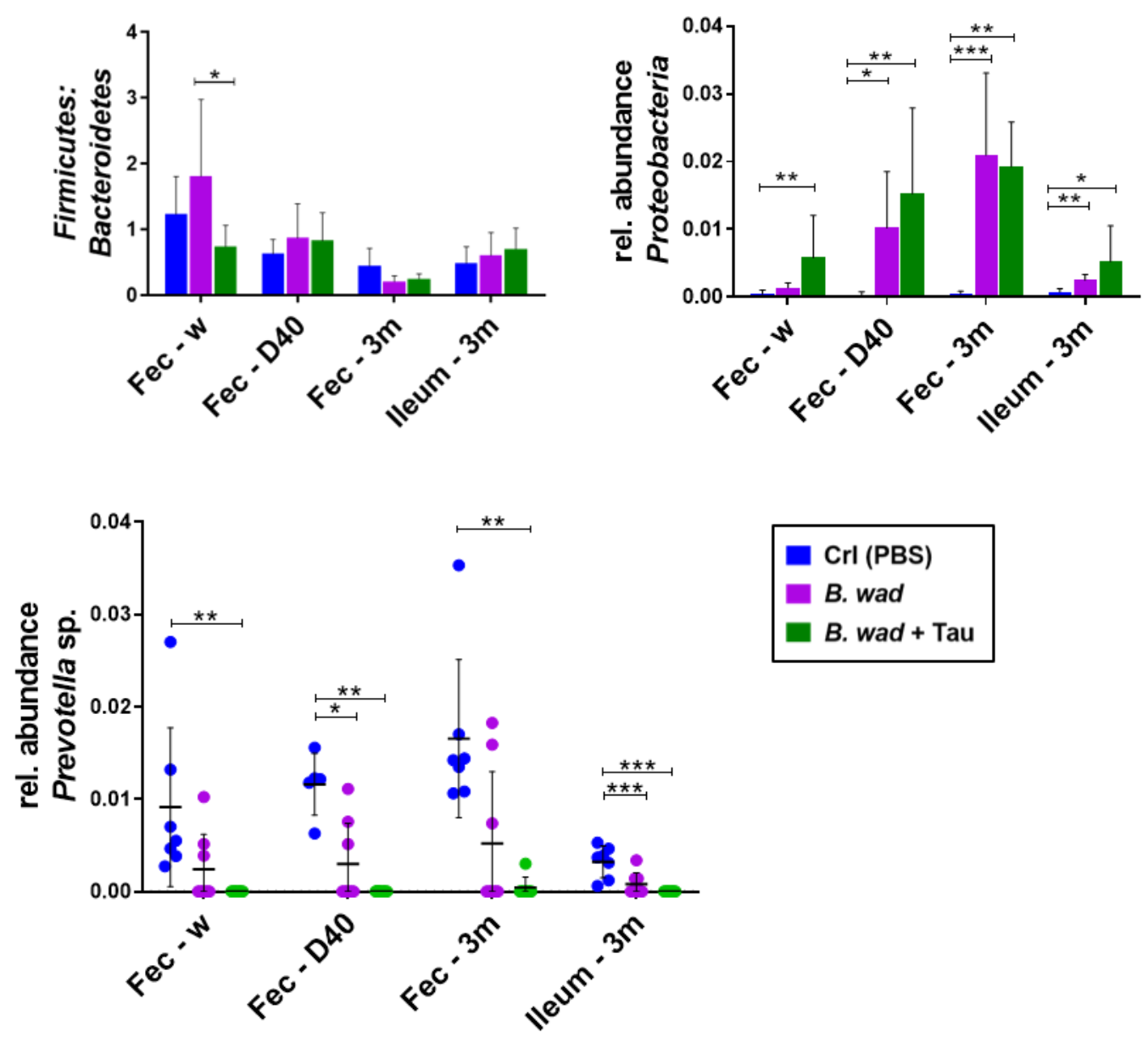
D
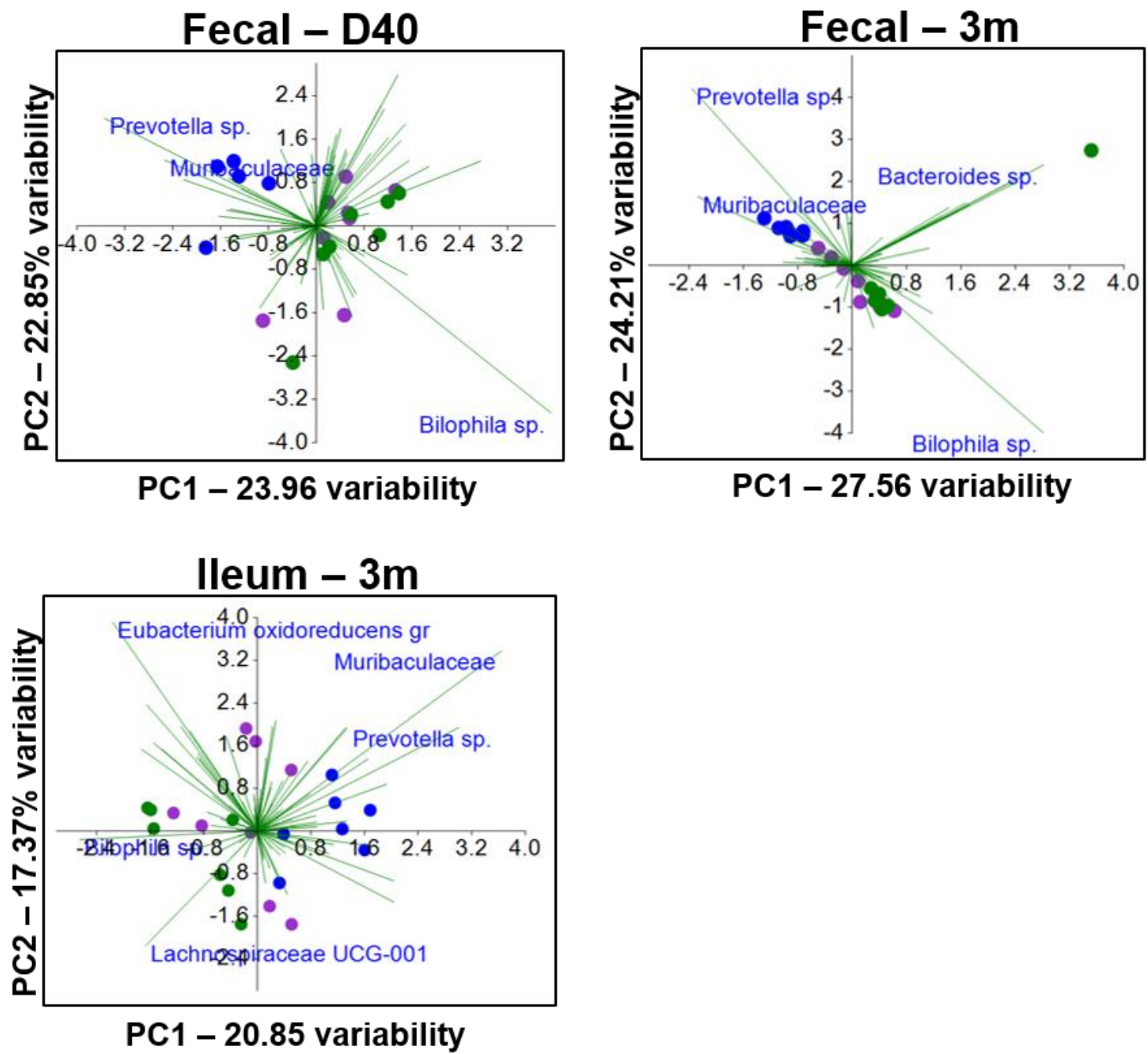
Figure 2. B. wadsworthia supplementation suppresses SI adenomas in $A p c^{M i n}$ mice.

A, Scatter plot comparing mean ( \pm SD) small intestinal (SI) and colon adenoma multiplicity of control $A p c^{M i n}$ offspring and two B. wadsworthia treatment groups following sacrifice at 3 months of age (PBS, $\mathrm{n}=5$, B. wadsworthia, $\mathrm{n}=8, B$.

wadsworthia + Tau, $\mathrm{n}=7) . \mathbf{B}$, Scatter plot comparing mean $( \pm \mathrm{SD})$ SI and colon adenoma burden, where each data point is calculated as the sum of estimated volumes of all adenomas found in an individual. C, Pearson's correlation method was used to show correlation between $B$. wadsworthia relative abundance in feces at 3 months of age and adenoma multiplicity among the two treatment groups $(n=14)$. D, Pearson's correlation showing the relationship between Prevotella sp. relative abundance in ileal epithelium scrapes and adenoma multiplicity in controls $(\mathrm{n}=7)$ and $\mathbf{E}$, treated groups $(\mathrm{n}=15) .{ }^{*} p<$ $0.05, * * P<0.01, * * * p<0.001 ;$ ANOVA with the Student Newman-Keuls method and Pearson's correlation method. Significant correlations have coefficient of determination $\left(\mathrm{r}^{2}\right)$ and $p$-value displayed on graphs.
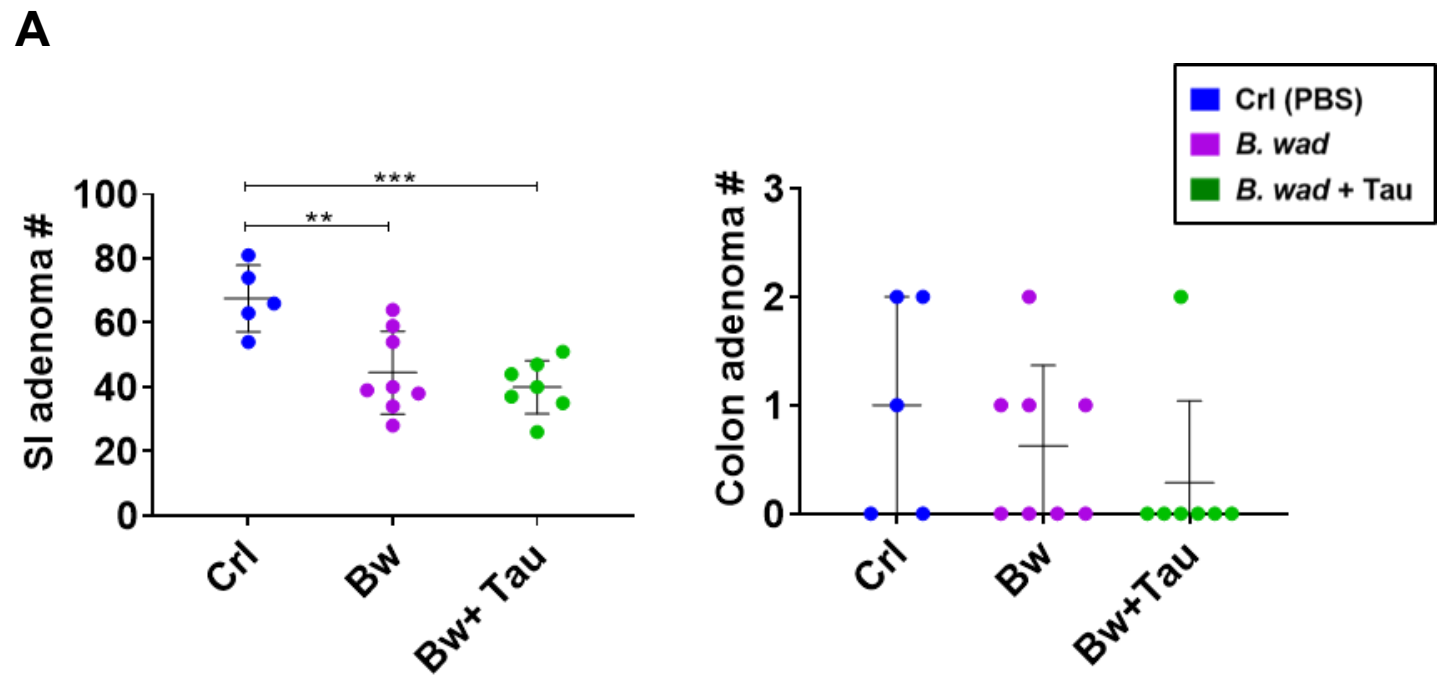
B
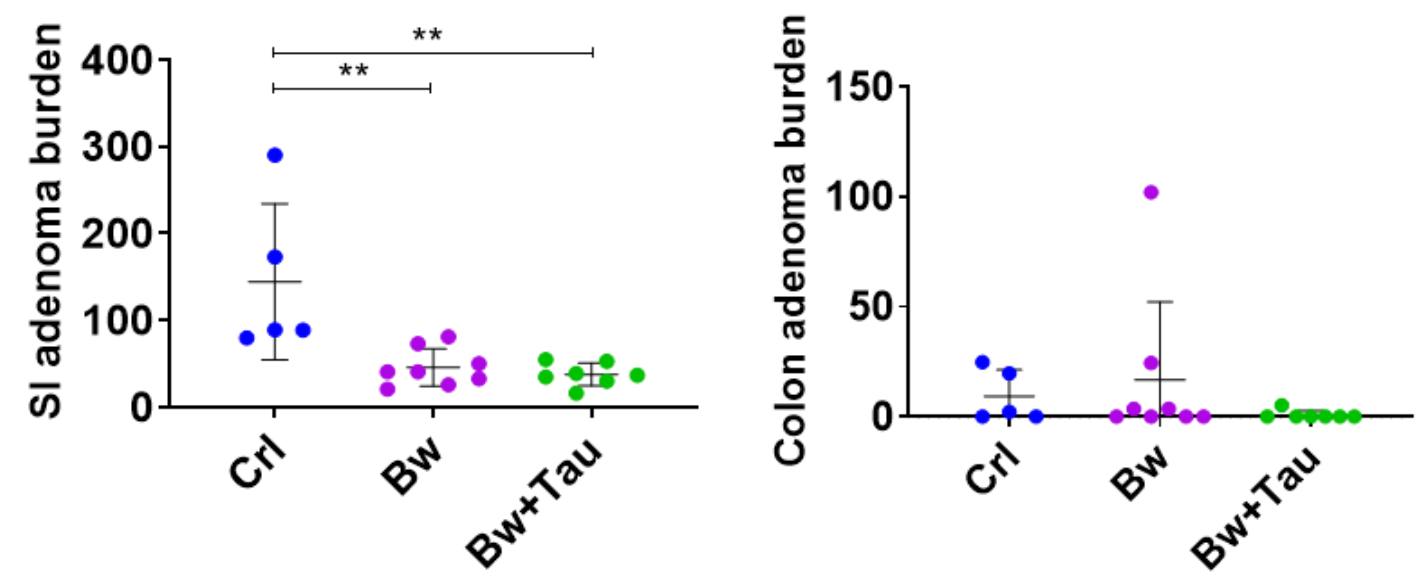

C

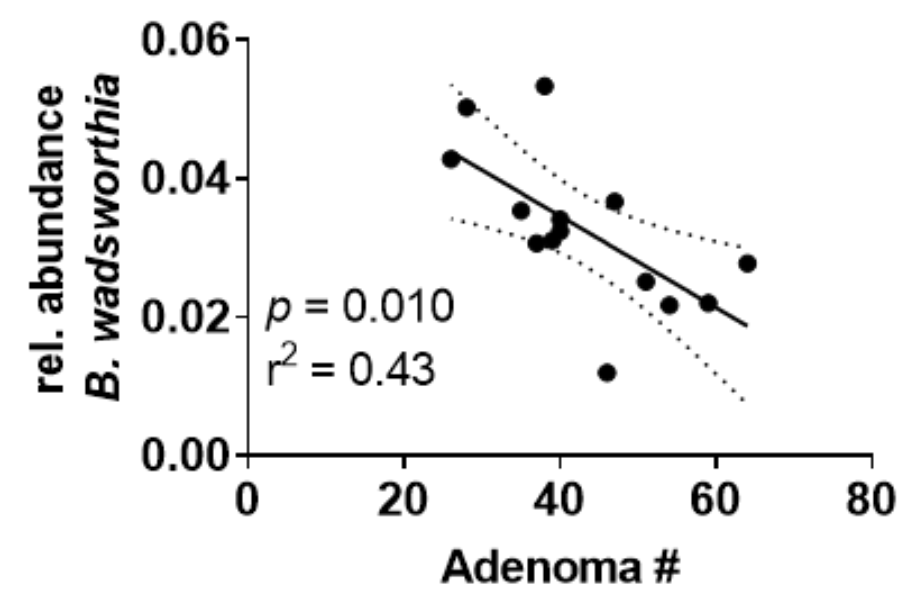


D
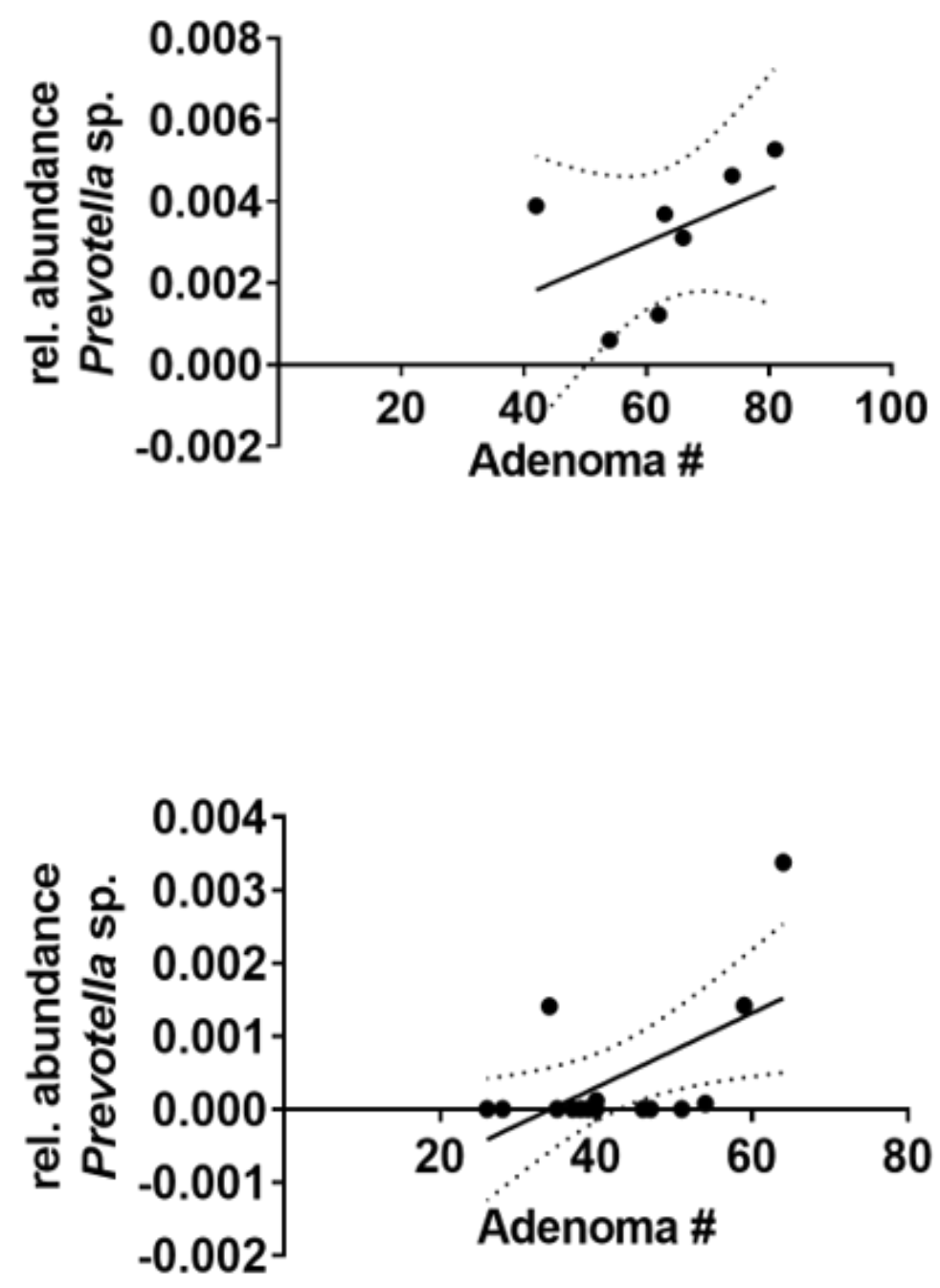
Figure 3. B. wadsworthia modulates the fecal and serum metabolome. A, PCA visualizing unsupervised clustering of untargeted fecal metabolite profiles of control $A p c^{M i n}$ mice $(\mathrm{n}=5)$ and $B$. wadsworthia + taurine $A p c^{\text {Min }}$ mice $(\mathrm{n}=5)$ at 3 months of age, and an associated pathway cloud plot, showing significantly modulated KEGG pathways based on differential metabolites. B, PCA visualizing unsupervised clustering of untargeted serum metabolite profiles of control $A p c^{M i n}$ mice $(\mathrm{n}=7)$ and $B$. wadsworthia + tau $A p c^{M i n}$ mice $(\mathrm{n}=7)$ at 3 months of age, and an associated pathway cloud plot, showing significantly modulated KEGG pathways based on differential metabolites. The $\mathrm{x}$-axis of the cloud plots indicate percentage of total metabolites mapped to a particular KEGG pathway that are differentially abundant between control and treatment groups. 
A
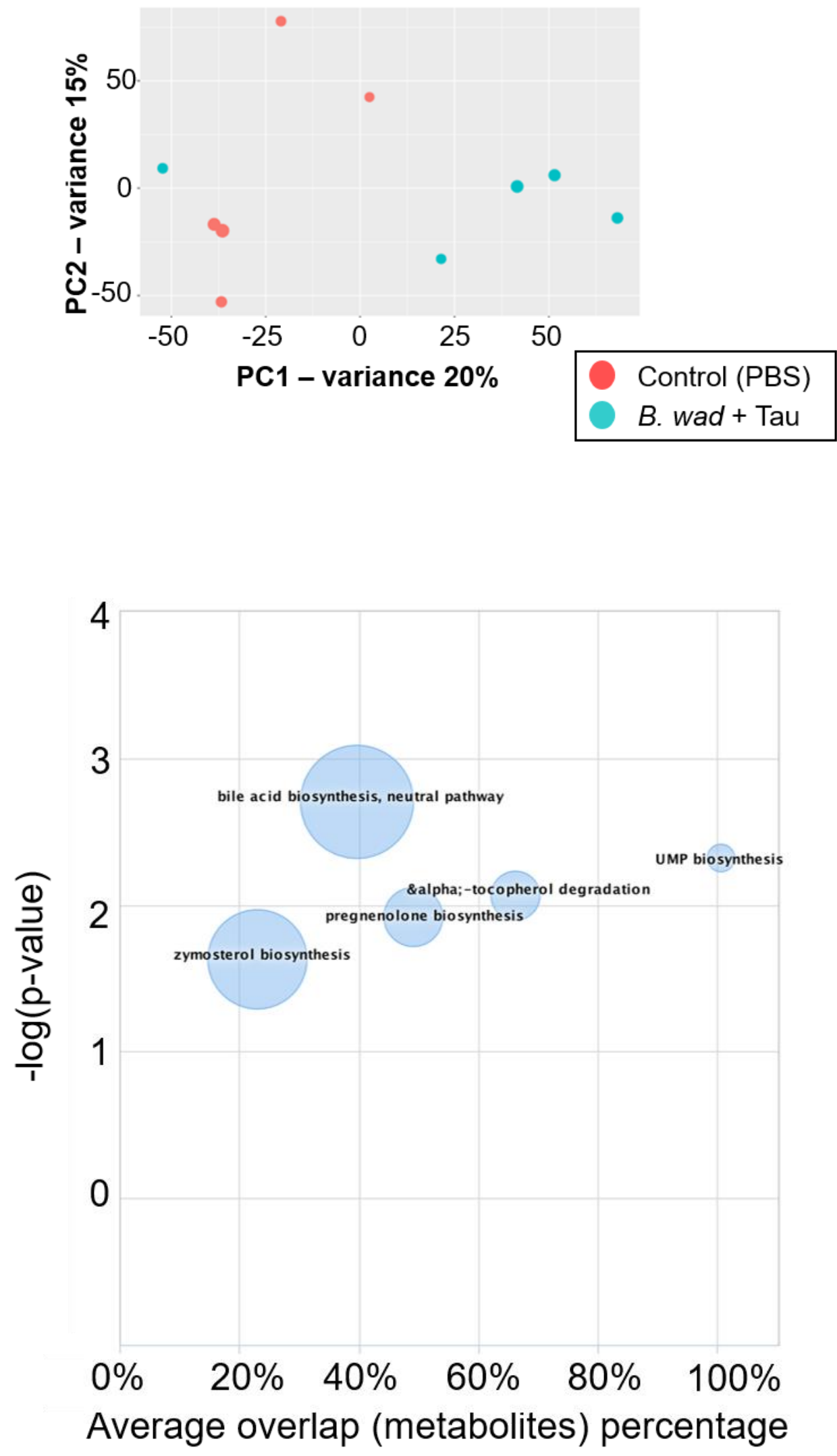
B
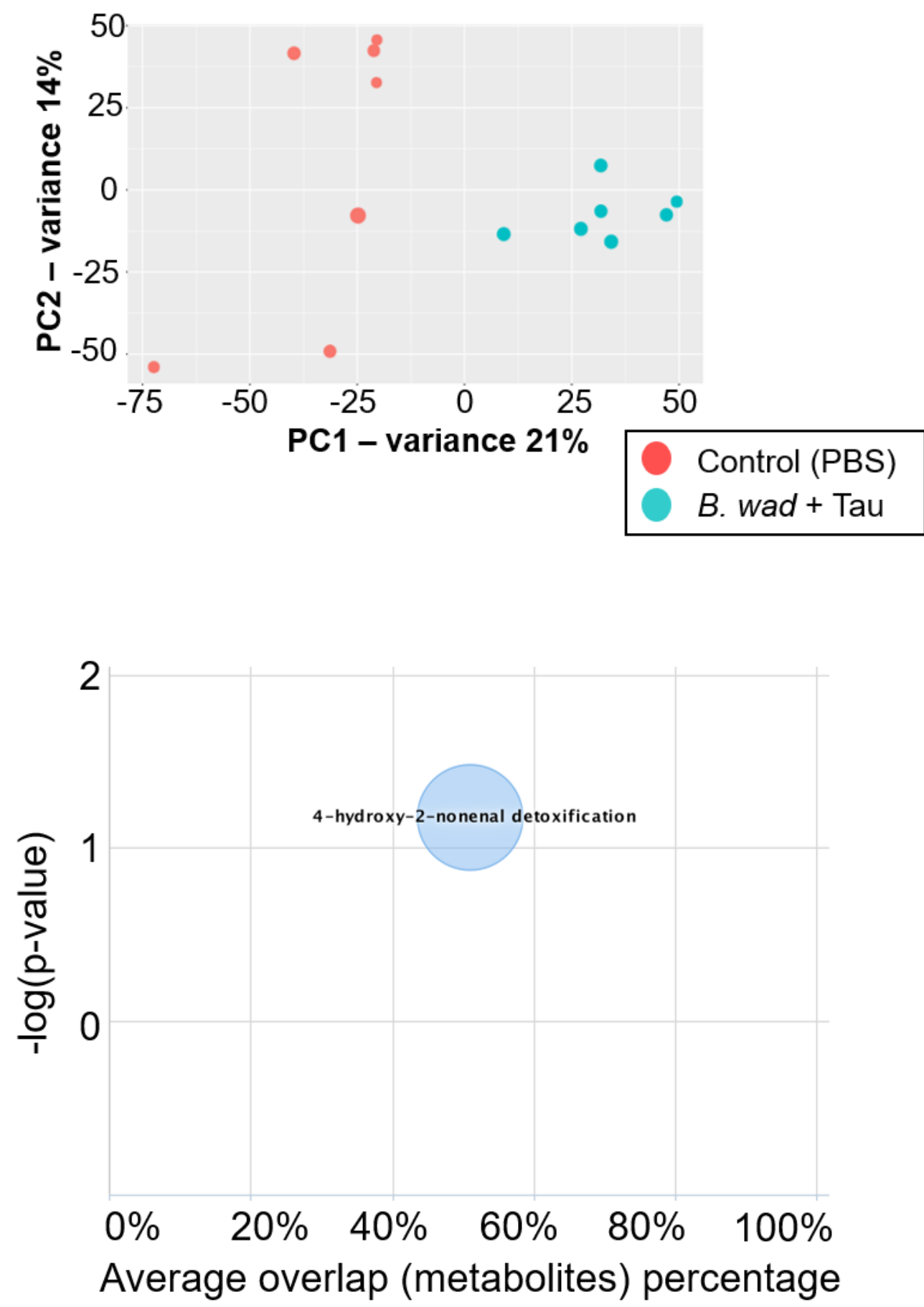
Figure 4. B. wadsworthia regulates fecal and serum bile acid composition. A, Scatter plot comparing mean $( \pm \mathrm{SD})$ relative abundances of detected fecal bile acids (control, $\mathrm{n}=$ 5; B. wadsworthia + taurine, $\mathrm{n}=5$ ) at 3 months of age. $\mathbf{B}$, Scatter plot comparing mean $( \pm \mathrm{SD})$ relative abundances of detected serum bile acids (control, $\mathrm{n}=7 ;$ B. wadsworthia + tau, $\mathrm{n}=7$ ) at 3 months of age. $* p<0.05, * * P<0.01$, $* * * p<0.001$; student's t-test.

A
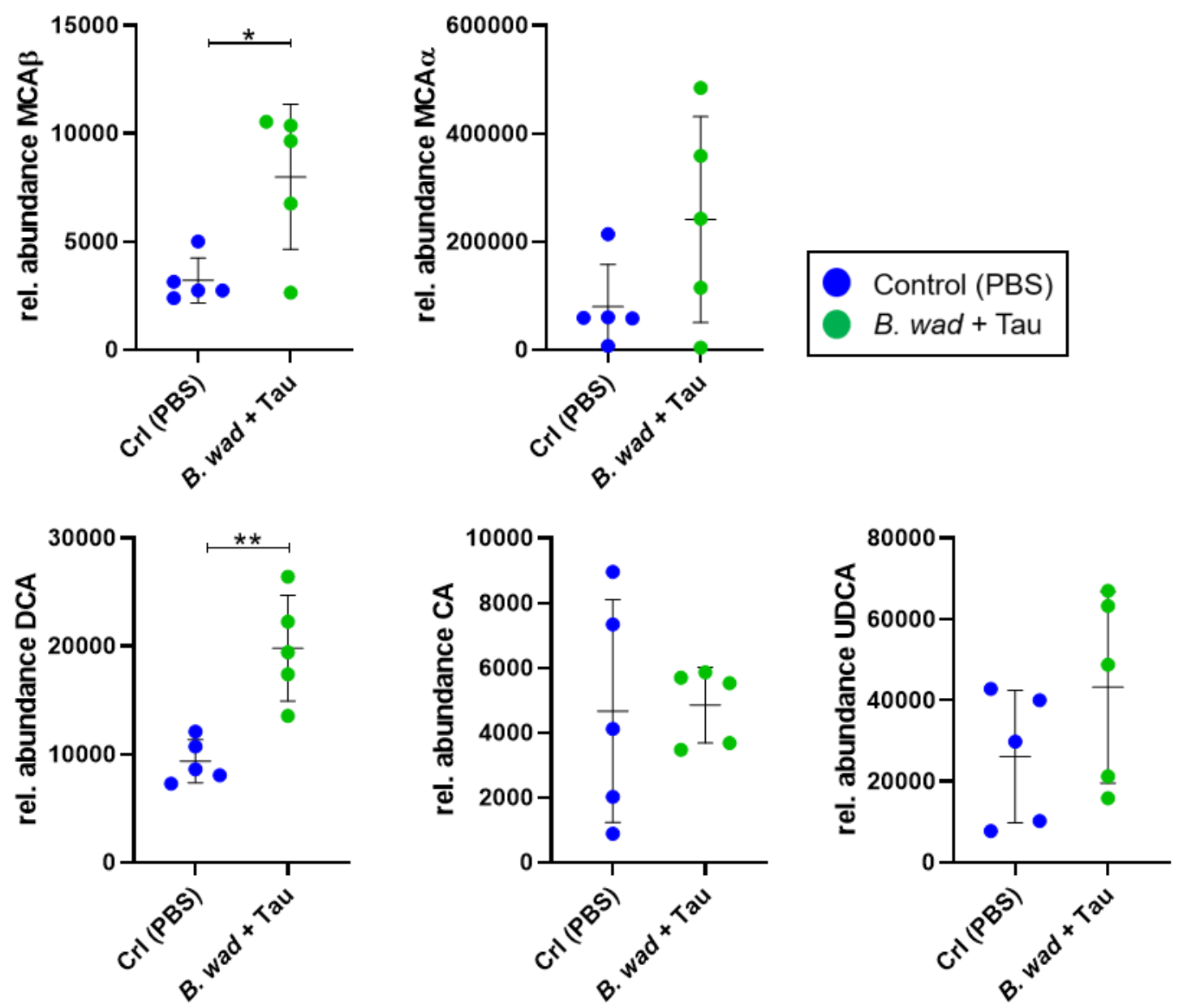
B
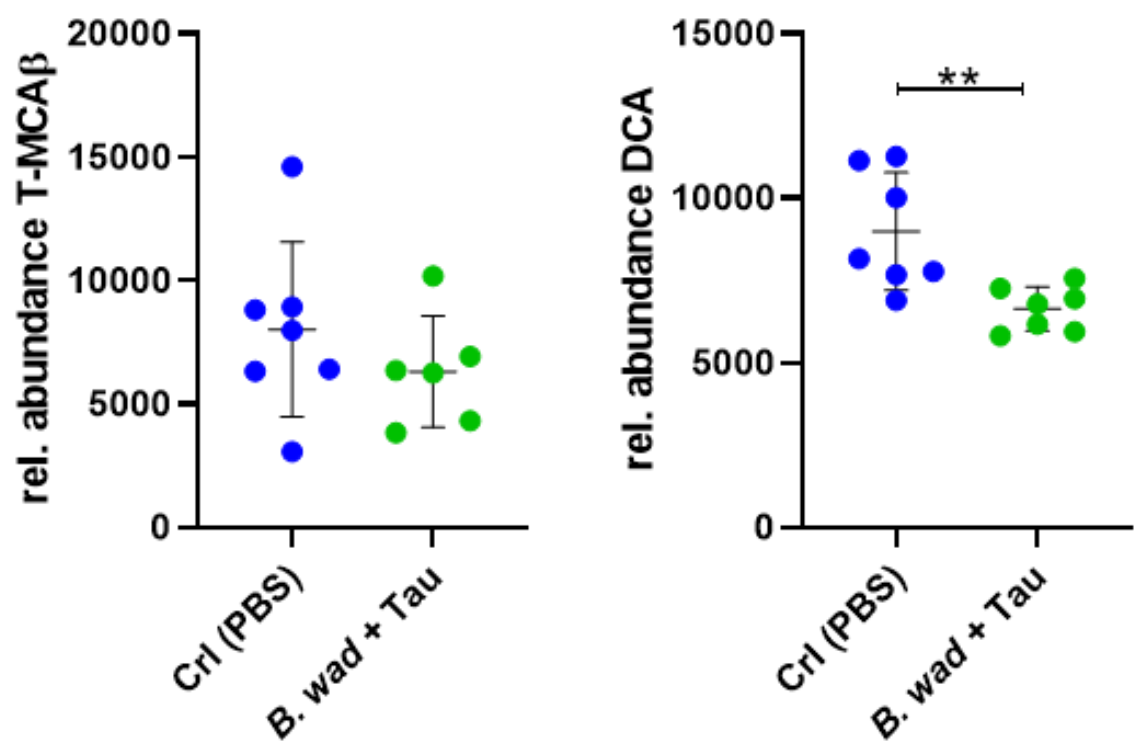

Control (PBS)

B. $w a d+\mathrm{Tau}$ 
Figure 5. Untargeted metabolites correlate with adenoma multiplicity and $B$.

wadsworthia abundance. A, Pearson's method was used to show significant correlations between untargeted fecal metabolites at 3 months of age and SI adenoma multiplicity in control and treated mice (control, $\mathrm{n}=5 ;$ B. wadsworthia + taurine, $\mathrm{n}=5$ ). $\mathbf{B}$, Fecal metabolites from panel A with concurrent correlations to fecal B. wadsworthia relative abundance in the treatment group are shown $($ B. wadsworthia + tau, $\mathrm{n}=5) . \mathbf{C}$, Pearson's method showing significant correlations between untargeted serum metabolites at 3 months of age and SI adenoma multiplicity (control, $\mathrm{n}=7 ;$ B. wadsworthia + tau, $\mathrm{n}=7$ ). D, Serum metabolites from panel C with concurrent correlations to fecal B. wadsworthia relative abundance in the treatment group are shown $(B$. wadsworthia + taurine, $\mathrm{n}=7)$. Metabolites with putative METLIN identification are labeled in y-axis, or are identified by mass-to-charge ratio/retention time $(\mathrm{m} / \mathrm{z}) / \mathrm{RT}$ if no identification is available. Pearson's coefficients of determination $\left(\mathrm{r}^{2}\right)$ and $p$ values are displayed on graphs. 
A
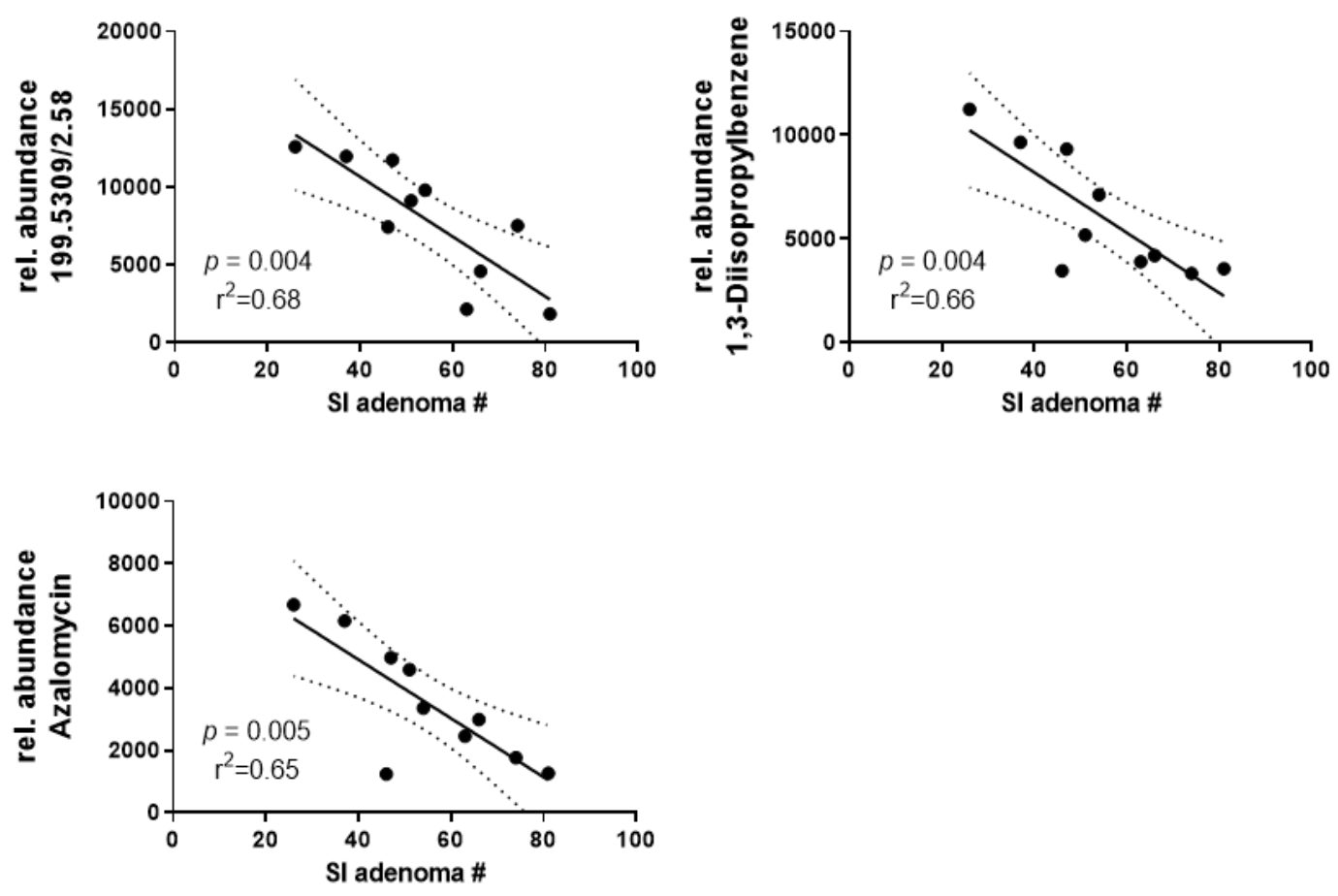

B
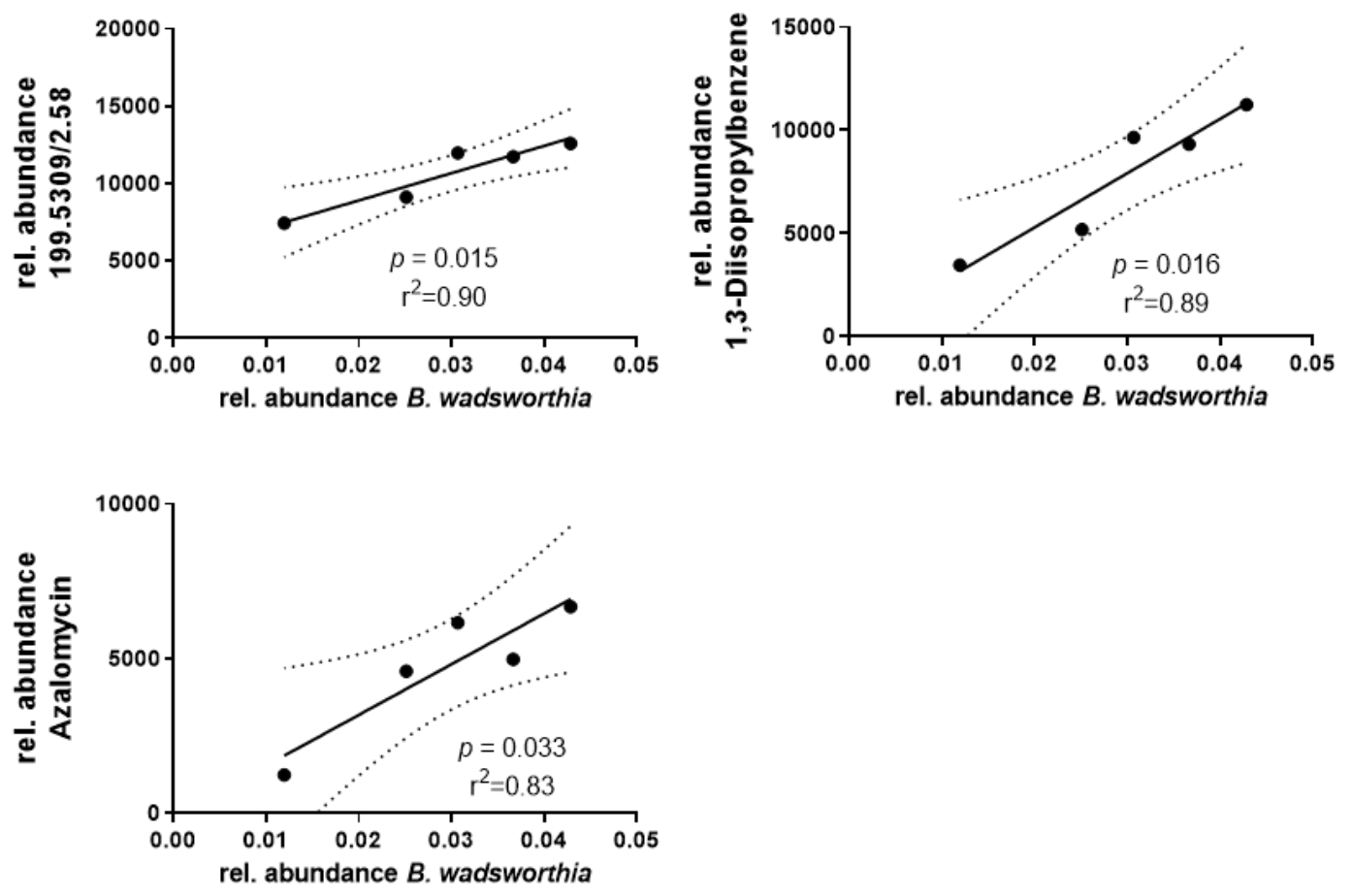
C
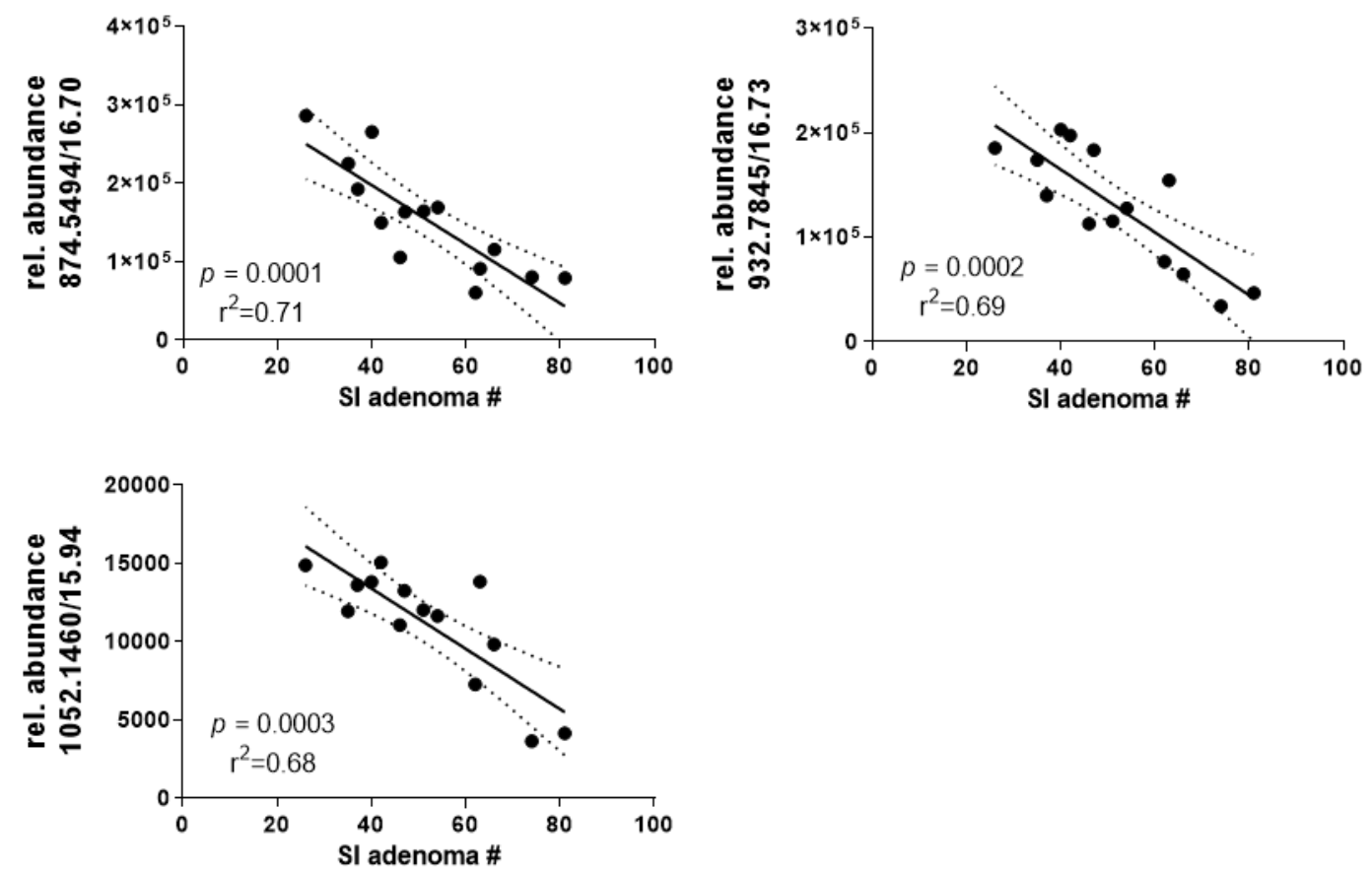

D
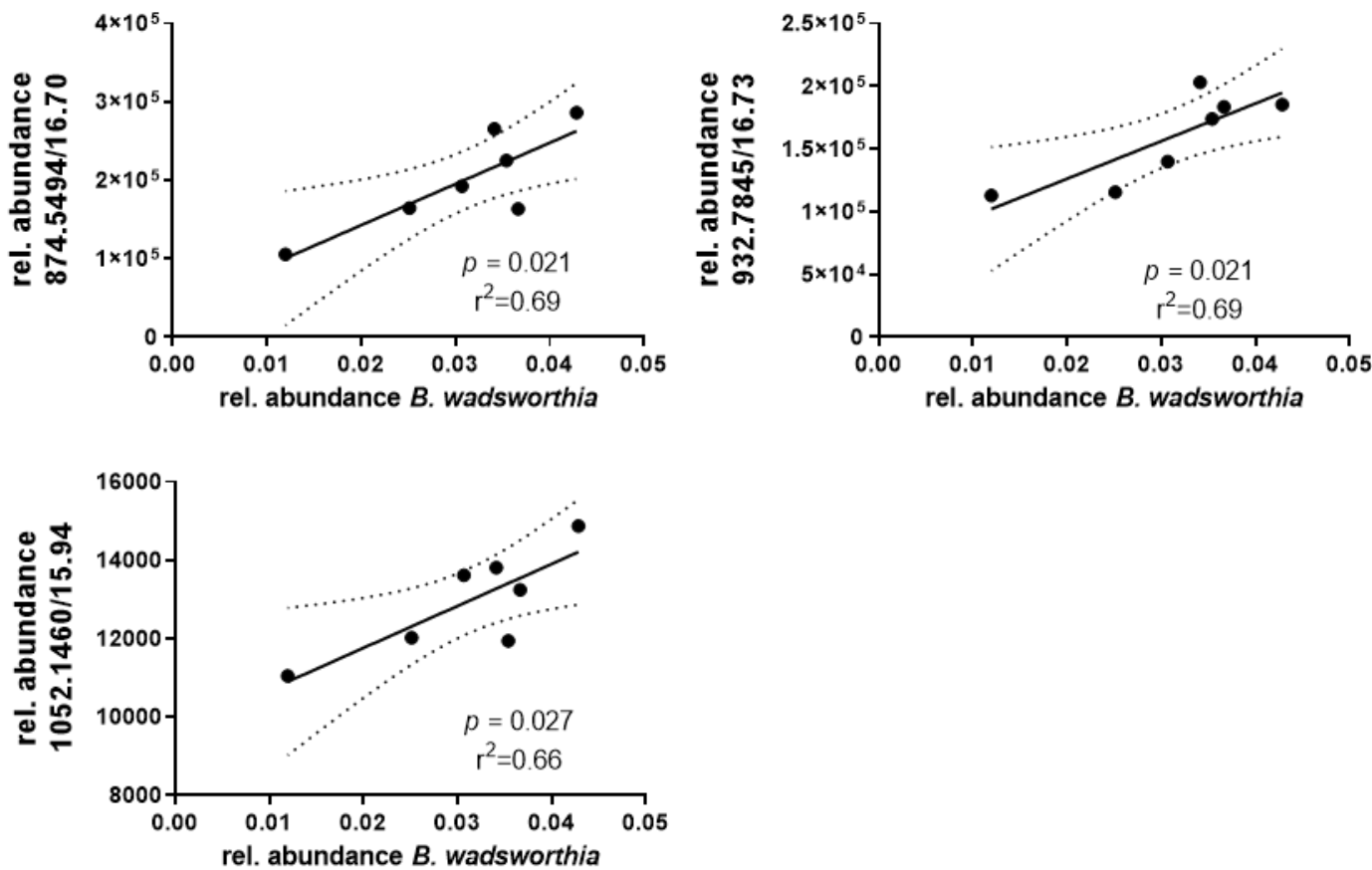
Supplementary Figure 1. Summary of SI and colonic adenoma sizes. Scatter plots comparing mean area $( \pm \mathrm{SD})$ of SI adenomas in four sections of the small intestine (S1S4) and the colon, after sacrifice at 3 months of age (controls, $\mathrm{n}=5 ;$ B. wadsworthia, $\mathrm{n}=$ $8, B$. wadsworthia + taurine). ${ }^{*} p<0.05,{ }^{*} P<<0.01,{ }^{* *} p<0.001$; ANOVA with the Student Newman-Keuls method.
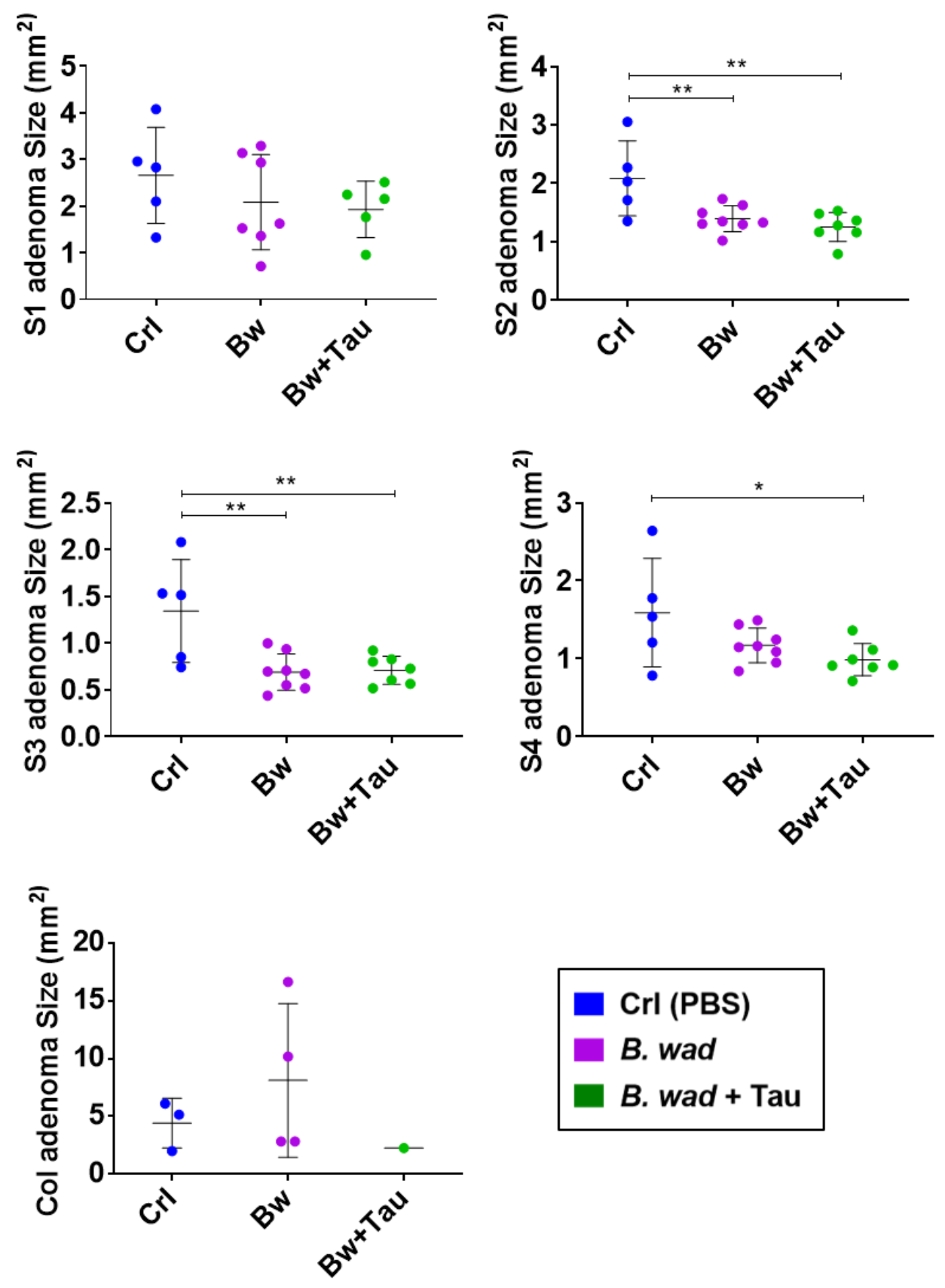
Supplementary Figure 2. PCA visualization of untargeted small intestinal content metabolite profiles. PCA visualizing unsupervised clustering of untargeted fecal metabolite profiles of control $A p c^{\text {Min }}$ mice $(\mathrm{n}=7)$ and $B$. wadsworthia + tau $A p c^{\text {Min }}$ mice $(n=7)$ at 3 months of age.

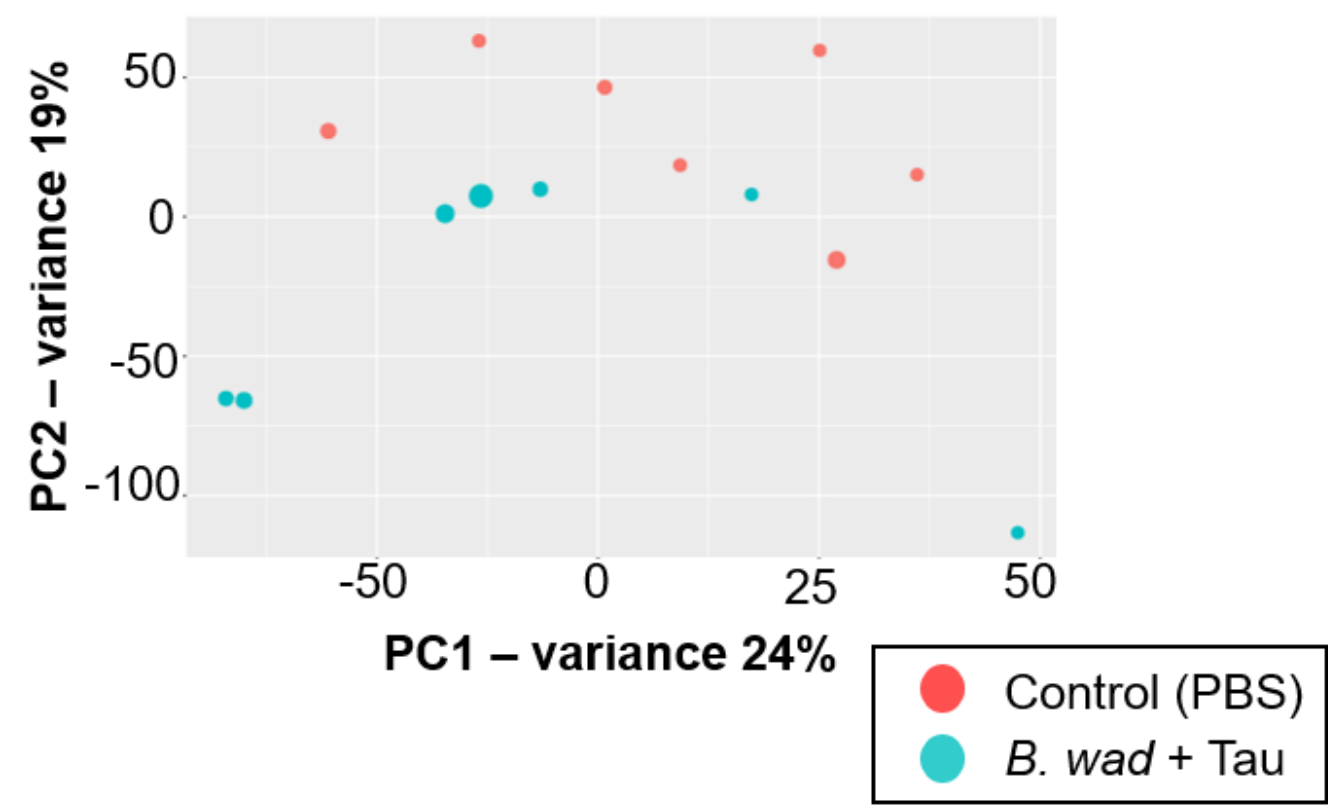


Supplementary Figure 3. Composition of detected bile acids in SI contents. Scatter plots comparing mean $( \pm \mathrm{SD}$ ) relative abundances of detected fecal bile acids (control, $\mathrm{n}$ $=7 ;$ B. wadsworthia + taurine, $\mathrm{n}=7$ ) at 3 months of age. Student's t-test.
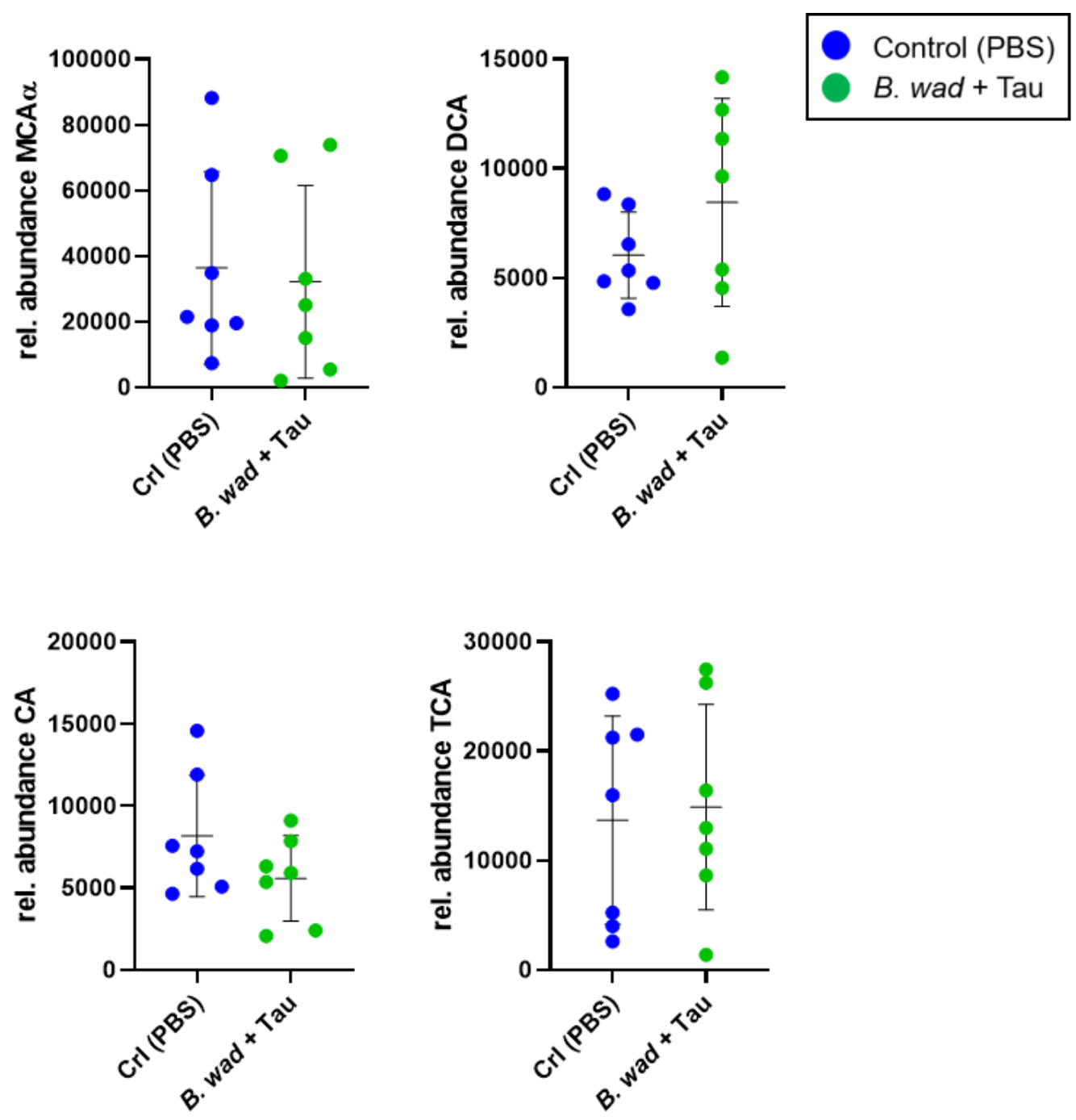


\section{Supplementary Figure 4. Untargeted fecal and serum metabolites correlate with}

adenoma multiplicity. A, Pearson's correlation method was used to show fecal

metabolites at 3 months of age with significant positive and negative correlations to SI adenoma multiplicity in control and treatment groups (control, $\mathrm{n}=5$, B. wadsworthia + taurine, $n=5$ ). B, Pearson's correlation method showing serum metabolites at 3 months of age with significant positive and negative correlations to SI adenoma multiplicity in control and treatment groups (control, $\mathrm{n}=7 ;$ B. wadsworthia + taurine, $\mathrm{n}=7$ ).

Metabolites are identified by mass-to-charge ratio/retention time (m/z)/RT. Pearson's coefficients of determination $\left(\mathrm{r}^{2}\right)$ and $p$ values are displayed on graphs.

A
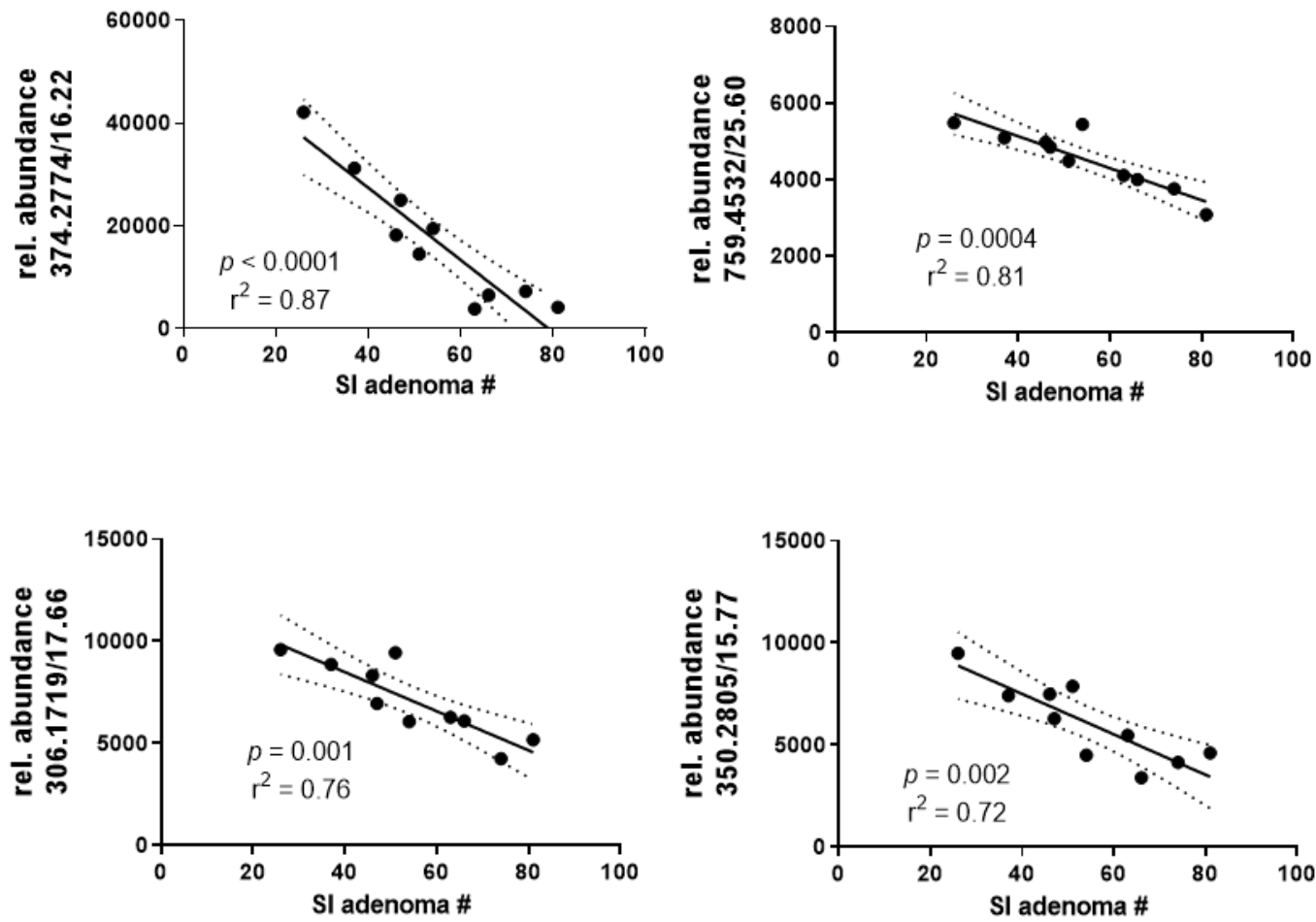

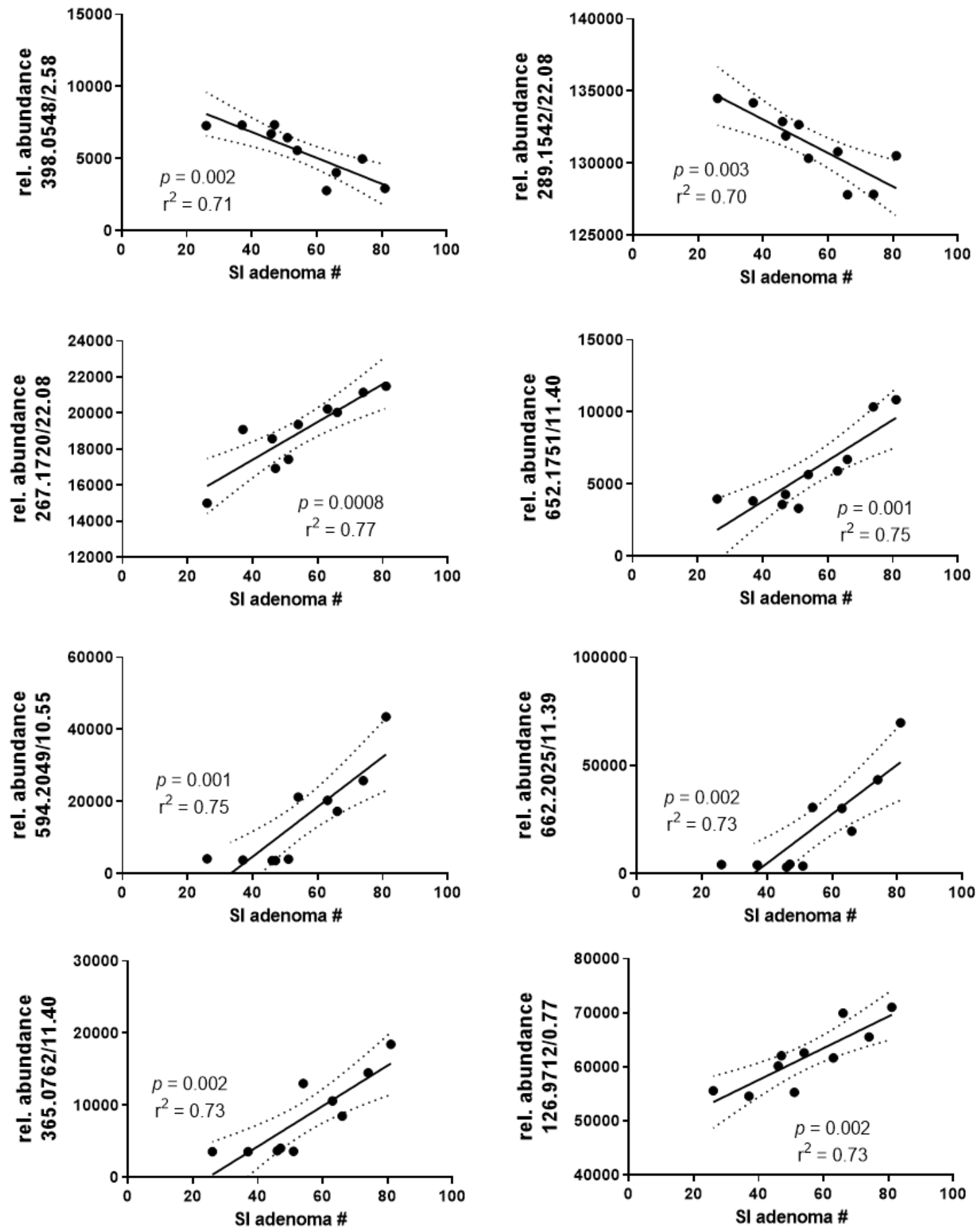
B
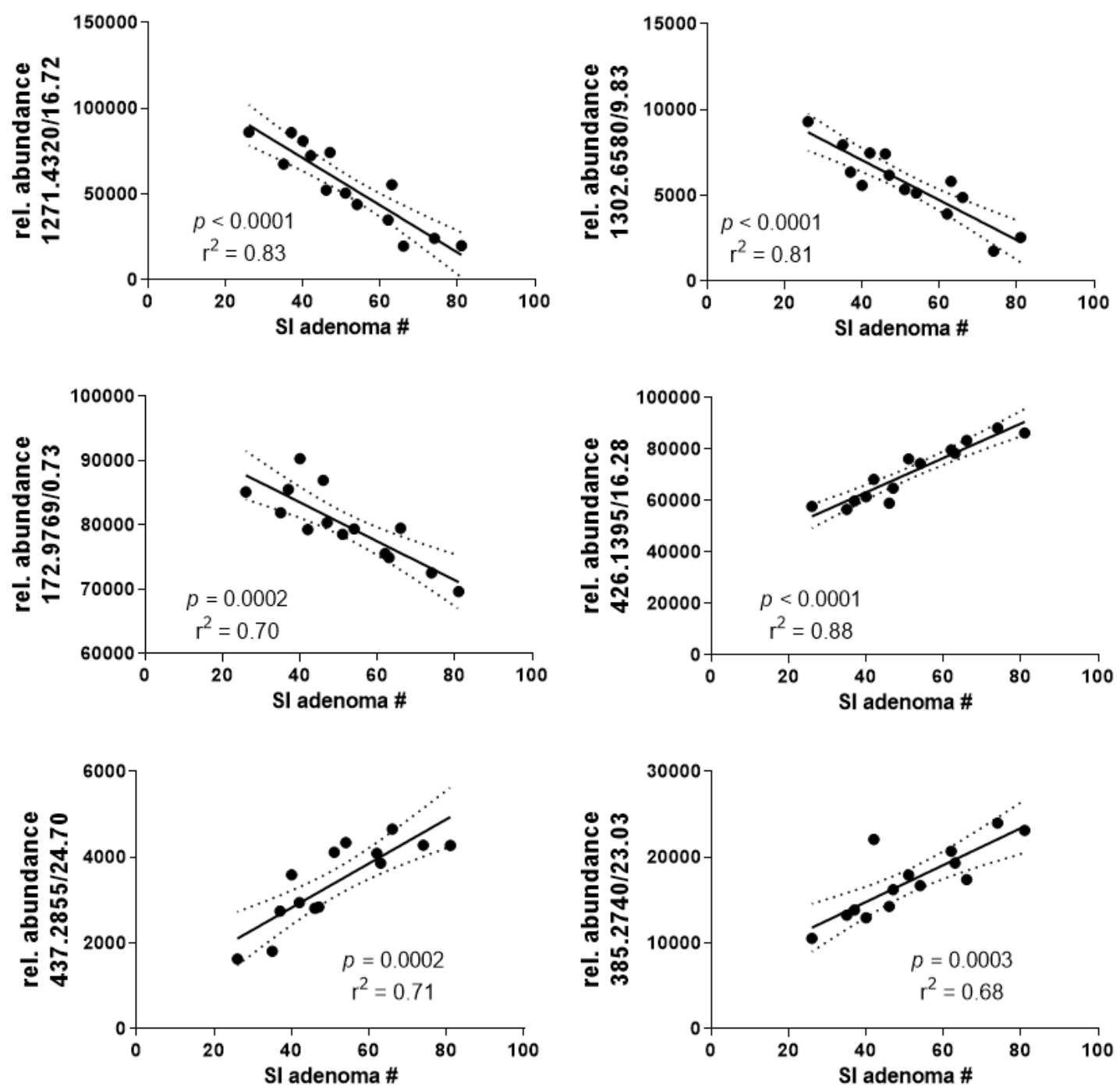


\section{Supplementary Figure 5. Untargeted metabolites correlate with B. wadsworthia}

relative abundance. A, Pearson's correlation shows fecal metabolites at 3 months of age with significant correlations to fecal relative abundance of $B$. wadsworthia (qPCR) in the treated group (B. wadsworthia + taurine, $\mathrm{n}=5$ ). B, Pearson's correlation shows serum metabolites at 3 months of age with significant correlations to qPCR-determined fecal relative abundance of $B$. wadsworthia in the treated group $(B$. wadsworthia + taurine, $\mathrm{n}=$ 7). Metabolites are identified by mass:charge ratio/retention time (m/z)/RT. Pearson's coefficients of determination $\left(\mathrm{r}^{2}\right)$ and $p$ values are displayed on graphs.

A
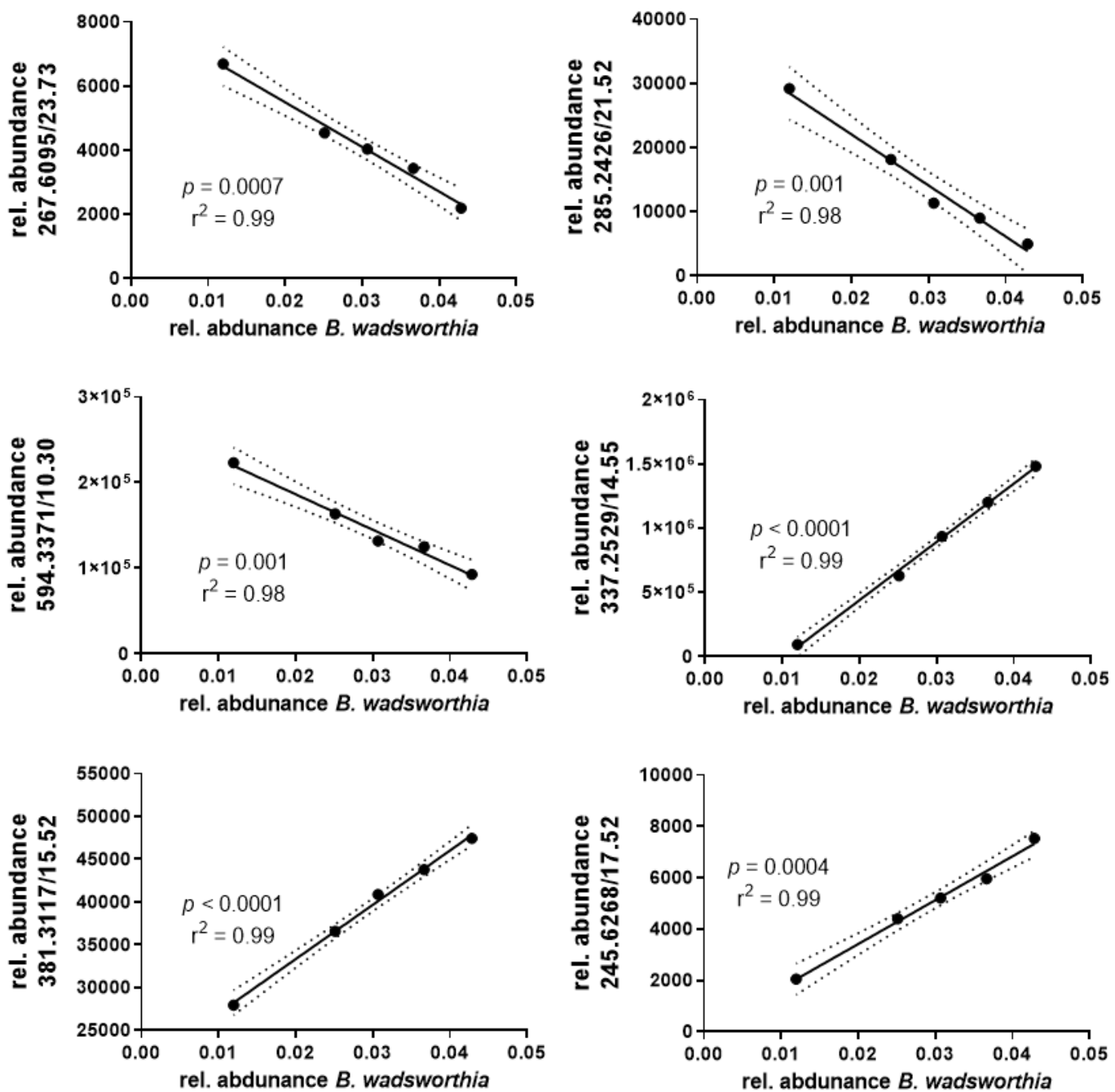
B
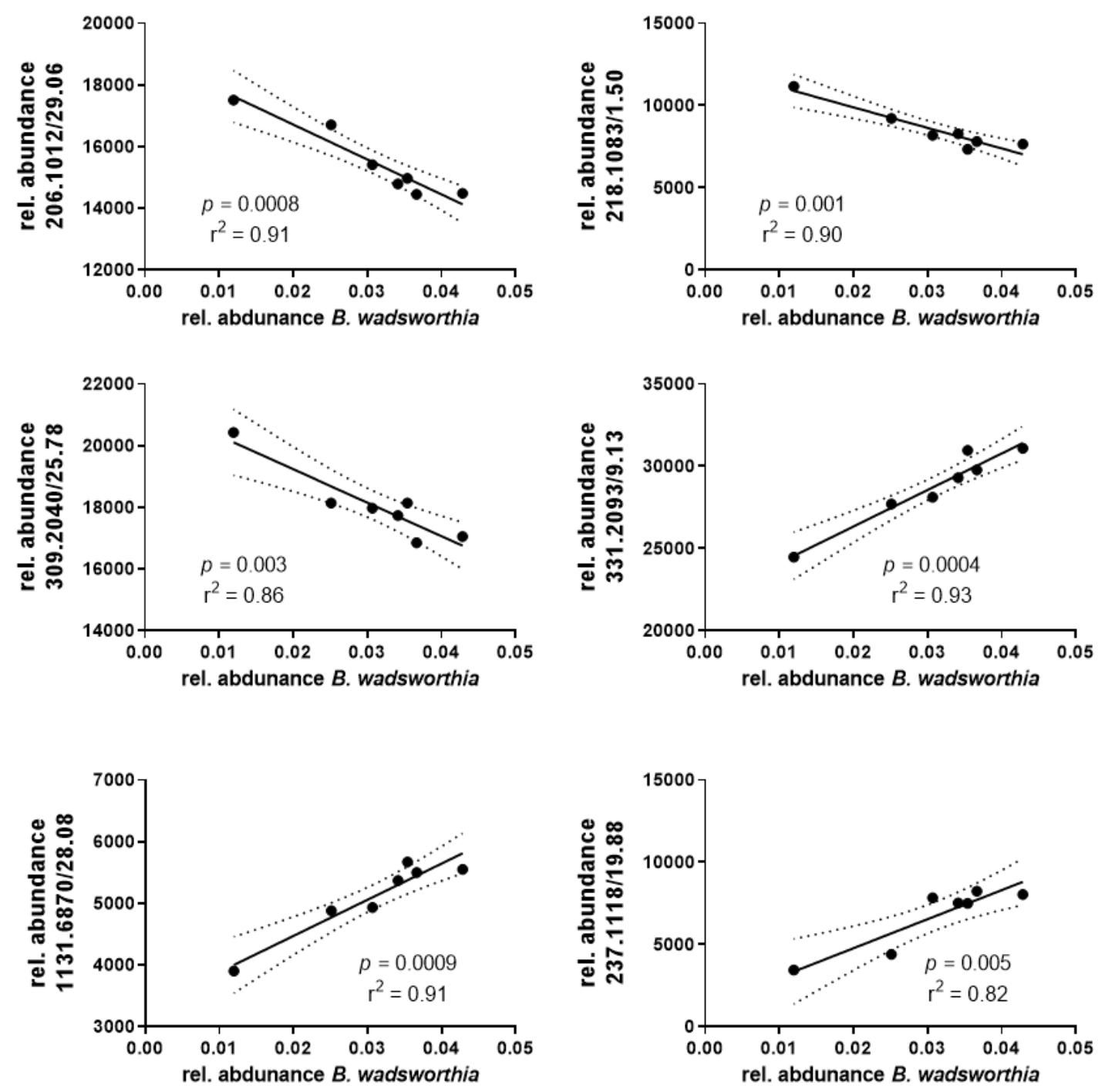


\section{Tables}

Supplementary Table 1. Significantly different OTUs in feces and ileal epithelium following B. wadsworthia supplementation

\begin{tabular}{|c|c|c|c|c|c|c|}
\hline & \multicolumn{2}{|c|}{ Significant OTUs (FDR < 0.05) } & \multirow[b]{2}{*}{ FDR } & \multicolumn{3}{|c|}{ Mean Relative Abundance ( \pm SEM) } \\
\hline Sample & Phylum & OTU & & $\begin{array}{l}\text { Control } \\
\text { (PBS) }\end{array}$ & B. wad & $\begin{array}{l}\text { B. wad }+ \\
\text { Tau }\end{array}$ \\
\hline \multirow{5}{*}{ Fecal-wean } & Proteobacteria & genus Bilophila & $1.7 \times 10^{-6}$ & ND & $\begin{array}{c}0.0011 \\
\pm 2.5 \times 10^{-4} \\
\end{array}$ & $\begin{array}{l}0.0051 \\
\pm .0019 \\
\end{array}$ \\
\hline & \multirow[t]{2}{*}{ Bacteroidetes } & family Muribaculaceae & $8.0 \times 10^{-4}$ & $\begin{array}{c}4.40 \times 10^{-4} \\
\pm 3.83 \times 10^{-5}\end{array}$ & $\begin{array}{c}1.40 \times 10^{-4} \\
\pm 7.36 \times 10^{-5}\end{array}$ & ND \\
\hline & & Genus Prevotella & .001 & $\begin{array}{c}.0091 \\
\pm .0033\end{array}$ & $\begin{array}{c}.0024 \\
\pm .0013\end{array}$ & $\begin{array}{r}9.99 \times 10^{-7} \\
\pm 9.99 \times 10^{-7}\end{array}$ \\
\hline & \multirow{2}{*}{ Firmicutes } & family Lachnospiraceae & .033 & $\begin{array}{c}.0316 \\
\pm .0072\end{array}$ & $\begin{array}{c}.0415 \\
\pm .0110\end{array}$ & $\begin{array}{c}.0087 \\
\pm .0041\end{array}$ \\
\hline & & genus Blautia & .046 & $\begin{array}{c}.0201 \\
\pm .0040 \\
\end{array}$ & $\begin{array}{c}.0189 \\
\pm .0058 \\
\end{array}$ & $\begin{array}{c}.0034 \\
\pm 7.71 \times 10^{-4} \\
\end{array}$ \\
\hline \multirow{5}{*}{ Fecal-D40 } & Proteobacteria & genus Bilophila & $6.1 \times 10^{-12}$ & ND & $\begin{array}{c}.0100 \\
\pm .0029\end{array}$ & $\begin{array}{c}.0151 \\
\pm .0048\end{array}$ \\
\hline & \multirow{2}{*}{ Bacteroidetes } & genus Prevotella & .011 & $\begin{array}{c}.0116 \\
\pm .0015\end{array}$ & $\begin{array}{c}.0030 \\
\pm .0016\end{array}$ & $\begin{array}{c}1.74 \times 10^{-5} \\
\pm 8.81 \times 10^{-6}\end{array}$ \\
\hline & & family Muribaculaceae & .011 & $\begin{array}{c}.0146 \\
\pm .0011\end{array}$ & $\begin{array}{c}.0058 \\
\pm .0017\end{array}$ & $\begin{array}{c}.0036 \\
\pm 5.16 \times 10^{-4}\end{array}$ \\
\hline & \multirow{2}{*}{ Firmicutes } & genus Lachnospiraceae NC2004 & $5.6 \times 10^{-4}$ & $\begin{array}{c}9.70 \times 10^{-4} \\
\pm 2.80 \times 10^{-4}\end{array}$ & $\begin{array}{c}1.40 \times 10^{-6} \\
\pm 1.40 \times 10^{-6}\end{array}$ & ND \\
\hline & & Eubacterium coprostanoligenes gr & .043 & $\begin{array}{c}.0026 \\
\pm .0011\end{array}$ & $\begin{array}{r}4.69 \times 10^{-5} \\
\pm 1.14 \times 10^{-5}\end{array}$ & $\begin{array}{r}1.07 \times 10^{-4} \\
\pm 1.06 \times 10^{-5}\end{array}$ \\
\hline
\end{tabular}




\begin{tabular}{|c|c|c|c|c|c|c|}
\hline & & genus Faecalibaculum & .045 & $\begin{array}{c}1.21 \times 10^{-4} \\
\pm 5.44 \times 10^{-5} \\
\end{array}$ & $\begin{array}{c}7.35 \times 10^{-4} \\
\pm 2.46 \times 10^{-4} \\
\end{array}$ & $\begin{array}{c}.0012 \\
\pm 4.23 \times 10^{-4} \\
\end{array}$ \\
\hline \multirow{5}{*}{ Fecal-3m } & Proteobacteria & genus Bilophila & $3.5 \times 10^{-13}$ & $\begin{array}{c}3.0 \times 10^{-6} \\
\pm 1.41 \times 10^{-6}\end{array}$ & $\begin{array}{c}.0207 \\
\pm .0043\end{array}$ & $\begin{array}{c}.0168 \\
\pm .0033\end{array}$ \\
\hline & \multirow{2}{*}{ Bacteroidetes } & genus Prevotella & .013 & $\begin{array}{c}.0166 \\
\pm .0032\end{array}$ & $\begin{array}{c}.0052 \\
\pm .0028\end{array}$ & $\begin{array}{c}4.32 \times 10^{-4} \\
\pm 4.31 \times 10^{-4}\end{array}$ \\
\hline & & family Muribaculaceae & .013 & $\begin{array}{c}2.69 \times 10^{-4} \\
\pm 2.18 \times 10^{-5}\end{array}$ & $\begin{array}{c}1.21 \times 10^{-4} \\
\pm 6.43 \times 10^{-5}\end{array}$ & $\begin{array}{c}6.14 \times 10^{-6} \\
\pm 6.14 \times 10^{-6}\end{array}$ \\
\hline & \multirow{2}{*}{ Firmicutes } & genus Oscillibacter & .021 & $\begin{array}{c}.0082 \\
\pm .0015 \\
\end{array}$ & $\begin{array}{c}.0033 \\
\pm 3.34 \times 10^{-4} \\
\end{array}$ & $\begin{array}{c}.0034 \\
\pm 4.38 \times 10^{-4} \\
\end{array}$ \\
\hline & & genus Lachnospiraceae NC2004 & .016 & $\begin{array}{c}3.69 \times 10^{-4} \\
\pm 1.97 \times 10^{-4}\end{array}$ & ND & ND \\
\hline \multirow{5}{*}{$\begin{array}{c}\text { Ileal } \\
\text { epithelium }\end{array}$} & Proteobacteria & genus Bilophila & $1.5 \times 10^{-10}$ & ND & $\begin{array}{c}.0017 \\
\pm 2.65 \times 10^{-4}\end{array}$ & $\begin{array}{c}.0026 \\
\pm 9.31 \times 10^{-4}\end{array}$ \\
\hline & Bacteroidetes & genus Prevotella & $3.4 \times 10^{-4}$ & $\begin{array}{c}.0032 \\
\pm 6.50 \times 10^{-4}\end{array}$ & $\begin{array}{c}7.99 \times 10^{-4} \\
\pm 4.29 \times 10^{-4}\end{array}$ & ND \\
\hline & \multirow{3}{*}{ Firmicutes } & genus Defluviitaleaceae UCG-011 & $8.1 \times 10^{-5}$ & $\begin{array}{c}3.55 \times 10^{-4} \\
\pm 5.83 \times 10^{-5}\end{array}$ & $\begin{array}{c}6.41 \times 10^{-5} \\
\pm 3.01 \times 10^{-5}\end{array}$ & ND \\
\hline & & genus Lachnospiraceae UCG-001 & .007 & $\begin{array}{c}1.29 \times 10^{-5} \\
\pm 1.29 \times 10^{-5}\end{array}$ & $\begin{array}{c}2.14 \times 10^{-4} \\
\pm 1.75 \times 10^{-4}\end{array}$ & $\begin{array}{c}.0013 \\
\pm 4.10 \times 10^{-4}\end{array}$ \\
\hline & & genus Lachnospiraceae NC2004 & .049 & $\begin{array}{c}2.5 \times 10^{-4} \\
\pm 9.46 \times 10^{-5}\end{array}$ & ND & ND \\
\hline
\end{tabular}

ND: Relative abundance was below detectable limits by 16 S sequencing

Statistical testing performed via ANOVA with Tukey's posthoc test 
Supplementary Table 2. Summary of significantly modulated serum

metabolites

\begin{tabular}{|l|l|l|l|l|}
\hline $\begin{array}{c}\text { Mass-charge/ } \\
\text { retention time } \\
\text { (mz/rt) }\end{array}$ & $\begin{array}{c}\text { Fold } \\
\text { change }\end{array}$ & q-value & $\begin{array}{c}\text { PP/DOWN in } \\
\text { Bwad + Tau }\end{array}$ & $\begin{array}{c}\text { Putative } \\
\text { Identification } \\
\text { METLIN ID }\end{array}$ \\
\hline $540.272 / 5.06$ & 1.94 & 0.0005 & DOWN & 155776 \\
\hline $1071.6115 / 24.84$ & 1.41 & 0.0105 & DOWN & NA \\
\hline $580.2927 / 24.84$ & 1.11 & 0.013 & DOWN & 163778 \\
\hline $122.0114 / 4.18$ & 1.17 & 0.0155 & DOWN & NA \\
\hline $517.3121 / 24.84$ & 1.34 & 0.0156 & DOWN & 138851 \\
\hline $268.1485 / 24.84$ & 1.27 & 0.017 & DOWN & 1137 \\
\hline $1098.6305 / 24.84$ & 1.33 & 0.0171 & DOWN & NA \\
\hline $713.3331 / 5.29$ & 2.09 & 0.0179 & DOWN & NA \\
\hline $267.6468 / 24.84$ & 1.29 & 0.018 & DOWN & NA \\
\hline $1084.1144 / 24.84$ & 1.57 & 0.0183 & DOWN & NA \\
\hline $425.1358 / 16.28$ & 1.28 & 0.0184 & DOWN & 1054226 \\
\hline $1073.6147 / 24.84$ & 1.46 & 0.0185 & DOWN & NA \\
\hline $1081.1046 / 24.84$ & 1.56 & 0.0185 & DOWN & NA \\
\hline $356.5267 / 24.84$ & 1.25 & 0.0185 & DOWN & NA \\
\hline $805.4683 / 24.84$ & 1.43 & 0.0185 & DOWN & 46653 \\
\hline $1070.601 / 24.84$ & 1.37 & 0.0187 & DOWN & NA \\
\hline $1075.624 / 24.84$ & 1.39 & 0.0188 & DOWN & NA \\
\hline $813.4452 / 24.84$ & 1.38 & 0.0188 & DOWN & 79016 \\
\hline $383.1162 / 0.61$ & 1.37 & 0.0188 & UP & 103590 \\
\hline $597.785 / 5.48$ & 2.49 & 0.0191 & DOWN & NA \\
\hline $516.8172 / 24.84$ & 1.45 & 0.0193 & DOWN & NA \\
\hline $516.3164 / 24.84$ & 1.45 & 0.0193 & DOWN & 986551 \\
\hline $276.6342 / 24.84$ & 1.21 & 0.0194 & DOWN & NA \\
\hline $278.1326 / 24.84$ & 1.2 & 0.0194 & DOWN & NA \\
\hline $356.861 / 24.84$ & 1.23 & 0.0194 & DOWN & NA \\
\hline $763.9831 / 24.84$ & 1.67 & 0.0195 & DOWN & NA \\
\hline $293.6623 / 26.61$ & 1.61 & 0.0196 & DOWN & NA \\
\hline $507.0073 / 6.5$ & 2.07 & 0.0196 & DOWN & 44119 \\
\hline $598.2862 / 5.47$ & 2.49 & 0.0196 & DOWN & NA \\
\hline $836.9495 / 24.84$ & 1.29 & 0.0197 & DOWN & NA \\
\hline $565.7791 / 24.84$ & 1.19 & 0.0197 & DOWN & NA \\
\hline
\end{tabular}




\begin{tabular}{|c|c|c|c|c|}
\hline $286.1402 / 24.84$ & 1.19 & 0.0197 & DOWN & NA \\
\hline $521.1976 / 23.52$ & 1.67 & 0.0198 & DOWN & 115714 \\
\hline $586.3098 / 24.84$ & 1.08 & 0.0198 & DOWN & 264086 \\
\hline $1114.5853 / 24.84$ & 1.38 & 0.0198 & DOWN & NA \\
\hline $1099.6345 / 24.84$ & 1.37 & 0.0198 & DOWN & NA \\
\hline $515.3134 / 24.84$ & 1.41 & 0.0199 & DOWN & 43148 \\
\hline $297.6478 / 24.84$ & 1.19 & 0.0199 & DOWN & NA \\
\hline $762.9799 / 24.84$ & 1.68 & 0.0199 & DOWN & NA \\
\hline $1043.1347 / 24.84$ & 1.79 & 0.0199 & DOWN & NA \\
\hline $814.453 / 24.84$ & 1.48 & 0.0199 & DOWN & NA \\
\hline $1082.6427 / 24.84$ & 1.6 & 0.02 & DOWN & NA \\
\hline $591.7527 / 16.28$ & 1.58 & 0.02 & DOWN & NA \\
\hline $233.5803 / 16.28$ & 1.25 & 0.02 & DOWN & NA \\
\hline $510.3562 / 26.48$ & 1.51 & 0.02 & DOWN & NA \\
\hline $274.6547 / 26.48$ & 1.41 & 0.02 & DOWN & NA \\
\hline $712.8304 / 5.29$ & 2.14 & 0.0201 & DOWN & NA \\
\hline $537.295 / 24.84$ & 1.24 & 0.0201 & DOWN & 69681 \\
\hline $518.3223 / 24.84$ & 1.3 & 0.0201 & DOWN & 40300 \\
\hline $401.3459 / 22.74$ & 1.39 & 0.0201 & DOWN & NA \\
\hline $478.3302 / 24.84$ & 1.34 & 0.0202 & DOWN & 40368 \\
\hline $515.8152 / 24.84$ & 1.4 & 0.0202 & DOWN & $\mathrm{NA}$ \\
\hline $1290.8032 / 24.84$ & 2.21 & 0.0202 & DOWN & NA \\
\hline $164.9298 / 0.69$ & 1.47 & 0.0202 & DOWN & NA \\
\hline $384.1198 / 0.59$ & 1.36 & 0.0202 & UP & NA \\
\hline $519.3257 / 24.84$ & 1.31 & 0.0202 & DOWN & NA \\
\hline $1247.3214 / 16.45$ & 1.32 & 0.0202 & DOWN & NA \\
\hline $1053.134 / 24.84$ & 1.61 & 0.0202 & DOWN & NA \\
\hline $1091.5983 / 24.84$ & 1.4 & 0.0202 & DOWN & 87624 \\
\hline $104.1069 / 24.84$ & 1.19 & 0.0202 & DOWN & NA \\
\hline $335.1256 / 23.57$ & 1.23 & 0.0202 & UP & 1011012 \\
\hline $222.5739 / 16.28$ & 1.26 & 0.0202 & DOWN & NA \\
\hline $426.1395 / 16.28$ & 1.28 & 0.0204 & DOWN & NA \\
\hline $277.6316 / 24.84$ & 1.19 & 0.0204 & DOWN & NA \\
\hline $524.2999 / 24.84$ & 1.35 & 0.0204 & DOWN & 40831 \\
\hline $1062.6191 / 24.84$ & 1.56 & 0.0204 & DOWN & NA \\
\hline $773.4672 / 24.84$ & 1.66 & 0.0204 & DOWN & NA \\
\hline $765.9883 / 24.84$ & 1.6 & 0.0204 & DOWN & NA \\
\hline $1331.2818 / 24.84$ & 1.68 & 0.0205 & DOWN & NA \\
\hline $1053.634 / 24.84$ & 1.91 & 0.0205 & DOWN & NA \\
\hline $498.3465 / 24.84$ & 1.38 & 0.0205 & DOWN & NA \\
\hline $481.9954 / 3.19$ & 1.97 & 0.0205 & DOWN & 1011802 \\
\hline $497.3436 / 24.84$ & 1.39 & 0.0205 & DOWN & NA \\
\hline $269.6436 / 24.84$ & 1.22 & 0.0206 & DOWN & NA \\
\hline
\end{tabular}




\begin{tabular}{|c|c|c|c|c|}
\hline $468.3457 / 28.88$ & 1.78 & 0.0206 & DOWN & 36726 \\
\hline $770.9656 / 24.84$ & 1.54 & 0.0206 & DOWN & NA \\
\hline $510.3561 / 26.13$ & 1.6 & 0.0207 & DOWN & NA \\
\hline $153.033 / 0.48$ & 1.14 & 0.0207 & DOWN & 62856 \\
\hline $241.9274 / 0.76$ & 1.37 & 0.0207 & DOWN & NA \\
\hline $496.3403 / 24.84$ & 1.41 & 0.0207 & DOWN & 1036728 \\
\hline $540.3064 / 21.91$ & 1.55 & 0.0208 & DOWN & 265004 \\
\hline $120.0527 / 16.28$ & 1.48 & 0.0208 & DOWN & NA \\
\hline $599.9491 / 4.21$ & 2.74 & 0.0208 & UP & NA \\
\hline $220.5531 / 16.27$ & 1.32 & 0.0208 & DOWN & NA \\
\hline $499.3492 / 24.84$ & 1.38 & 0.0208 & DOWN & NA \\
\hline $525.2989 / 24.84$ & 1.36 & 0.0209 & DOWN & NA \\
\hline $203.0526 / 0.6$ & 1.38 & 0.0209 & UP & NA \\
\hline $518.3248 / 21.91$ & 1.77 & 0.0209 & DOWN & 40300 \\
\hline $1014.659 / 24.84$ & 2.11 & 0.0209 & DOWN & NA \\
\hline $766.9913 / 24.84$ & 1.61 & 0.0209 & DOWN & NA \\
\hline $520.3286 / 24.84$ & 1.28 & 0.021 & DOWN & NA \\
\hline $417.6388 / 16.28$ & 1.33 & 0.021 & DOWN & NA \\
\hline $796.973 / 24.84$ & 1.37 & 0.021 & DOWN & NA \\
\hline $817.3173 / 16.28$ & 1.39 & 0.021 & DOWN & $\mathrm{NA}$ \\
\hline $385.274 / 23.03$ & 1.45 & 0.021 & DOWN & 986657 \\
\hline $511.3595 / 26.48$ & 1.41 & 0.021 & DOWN & NA \\
\hline $1061.1104 / 24.84$ & 1.53 & 0.021 & DOWN & 95547 \\
\hline $763.4816 / 24.84$ & 1.66 & 0.0211 & DOWN & 81082 \\
\hline $283.6599 / 26.48$ & 1.38 & 0.0211 & DOWN & NA \\
\hline $1085.1189 / 24.84$ & 1.51 & 0.0211 & DOWN & 95548 \\
\hline $1007.3956 / 16.28$ & 1.68 & 0.0211 & DOWN & NA \\
\hline $764.484 / 24.84$ & 1.55 & 0.0211 & DOWN & NA \\
\hline $849.2747 / 16.28$ & 1.38 & 0.0211 & DOWN & NA \\
\hline $1044.6372 / 24.84$ & 1.71 & 0.0211 & DOWN & NA \\
\hline $1013.6553 / 24.84$ & 2.07 & 0.0211 & DOWN & NA \\
\hline $1048.1488 / 24.84$ & 1.85 & 0.0211 & DOWN & NA \\
\hline $213.067 / 16.28$ & 1.28 & 0.0211 & DOWN & 1021606 \\
\hline $481.3124 / 25.57$ & 1.62 & 0.0212 & DOWN & NA \\
\hline $1015.6619 / 24.84$ & 2.1 & 0.0212 & DOWN & NA \\
\hline $766.4897 / 24.84$ & 1.63 & 0.0212 & DOWN & NA \\
\hline $1015.1592 / 24.84$ & 2.14 & 0.0212 & DOWN & NA \\
\hline $1021.6347 / 24.84$ & 1.8 & 0.0212 & DOWN & NA \\
\hline $1149.629 / 24.84$ & 1.39 & 0.0212 & DOWN & NA \\
\hline $848.0777 / 16.7$ & 1.84 & 0.0212 & UP & NA \\
\hline $362.2774 / 23.04$ & 1.52 & 0.0212 & DOWN & NA \\
\hline $292.1484 / 28.08$ & 1.31 & 0.0212 & DOWN & NA \\
\hline $772.9668 / 24.84$ & 1.59 & 0.0212 & DOWN & NA \\
\hline
\end{tabular}




\begin{tabular}{|c|c|c|c|c|}
\hline $314.0299 / 24.84$ & 1.2 & 0.0212 & DOWN & 989498 \\
\hline $524.8015 / 24.84$ & 1.34 & 0.0212 & DOWN & NA \\
\hline $792.4602 / 24.84$ & 1.36 & 0.0212 & DOWN & NA \\
\hline $226.9627 / 0.76$ & 1.31 & 0.0212 & DOWN & 92479 \\
\hline $1081.6408 / 24.84$ & 1.65 & 0.0212 & DOWN & NA \\
\hline $1089.5914 / 24.84$ & 1.55 & 0.0212 & DOWN & $\mathrm{NA}$ \\
\hline $591.2512 / 16.28$ & 1.56 & 0.0213 & DOWN & 80004 \\
\hline $275.6332 / 24.84$ & 1.18 & 0.0213 & DOWN & NA \\
\hline $1339.7633 / 24.84$ & 1.23 & 0.0213 & DOWN & NA \\
\hline $1040.1271 / 24.84$ & 1.77 & 0.0213 & DOWN & NA \\
\hline $771.9663 / 24.84$ & 1.62 & 0.0213 & DOWN & NA \\
\hline $1216.1961 / 16.7$ & 2.11 & 0.0213 & UP & NA \\
\hline $1063.6188 / 24.84$ & 1.74 & 0.0213 & DOWN & NA \\
\hline $801.9441 / 24.84$ & 1.4 & 0.0213 & DOWN & NA \\
\hline $336.1291 / 23.57$ & 1.25 & 0.0213 & UP & NA \\
\hline $525.7998 / 24.84$ & 1.31 & 0.0213 & DOWN & $\mathrm{NA}$ \\
\hline $773.9678 / 24.84$ & 1.51 & 0.0213 & DOWN & NA \\
\hline $286.0959 / 16.28$ & 1.27 & 0.0213 & DOWN & 1032387 \\
\hline $993.6801 / 24.84$ & 1.85 & 0.0213 & DOWN & NA \\
\hline $482.4972 / 3.19$ & 1.8 & 0.0213 & DOWN & NA \\
\hline $1011.6502 / 24.84$ & 2.04 & 0.0213 & DOWN & NA \\
\hline $294.0036 / 0.59$ & 1.48 & 0.0213 & UP & 999439 \\
\hline $1064.6192 / 24.84$ & 1.65 & 0.0213 & DOWN & NA \\
\hline $925.2933 / 16.28$ & 1.43 & 0.0214 & DOWN & 985265 \\
\hline $929.2358 / 16.28$ & 1.24 & 0.0216 & DOWN & NA \\
\hline $1247.1783 / 16.44$ & 1.34 & 0.0217 & DOWN & NA \\
\hline $263.5299 / 4.18$ & 1.08 & 0.0217 & DOWN & NA \\
\hline $818.3205 / 16.28$ & 1.44 & 0.0218 & DOWN & NA \\
\hline $1113.5828 / 24.84$ & 1.33 & 0.0218 & DOWN & $\mathrm{NA}$ \\
\hline $268.6494 / 24.84$ & 1.27 & 0.0218 & DOWN & NA \\
\hline $548.3717 / 26.6$ & 1.61 & 0.0219 & DOWN & 40011 \\
\hline $1092.6047 / 24.84$ & 1.39 & 0.0219 & DOWN & NA \\
\hline $1011.1482 / 24.84$ & 2.07 & 0.0219 & DOWN & $\mathrm{NA}$ \\
\hline $1064.1199 / 24.84$ & 1.58 & 0.0219 & DOWN & NA \\
\hline $1014.1566 / 24.84$ & 2.29 & 0.022 & DOWN & NA \\
\hline $1012.1502 / 24.84$ & 1.97 & 0.022 & DOWN & NA \\
\hline $1304.8127 / 24.84$ & 1.78 & 0.022 & DOWN & NA \\
\hline $222.054 / 16.28$ & 1.2 & 0.0221 & DOWN & NA \\
\hline $522.7971 / 24.84$ & 1.32 & 0.0221 & DOWN & NA \\
\hline $293.1021 / 16.28$ & 1.68 & 0.0222 & DOWN & 985231 \\
\hline $800.9439 / 24.84$ & 1.39 & 0.0223 & DOWN & NA \\
\hline $1332.2853 / 24.84$ & 1.65 & 0.0223 & DOWN & NA \\
\hline $500.3517 / 24.84$ & 1.28 & 0.0223 & DOWN & NA \\
\hline
\end{tabular}




\begin{tabular}{|c|c|c|c|c|}
\hline $480.309 / 25.57$ & 1.7 & 0.0223 & DOWN & 40282 \\
\hline $412.2097 / 25.71$ & 1.33 & 0.0223 & DOWN & 1002460 \\
\hline $543.2735 / 24.84$ & 1.19 & 0.0224 & DOWN & 57626 \\
\hline $606.3089 / 25.7$ & 1.4 & 0.0225 & DOWN & 197378 \\
\hline $1061.612 / 24.84$ & 1.54 & 0.0225 & DOWN & NA \\
\hline $804.3131 / 16.28$ & 1.43 & 0.0225 & DOWN & NA \\
\hline $148.0686 / 16.28$ & 1.38 & 0.0226 & DOWN & NA \\
\hline $771.4673 / 24.84$ & 1.6 & 0.0226 & DOWN & NA \\
\hline $1291.3036 / 24.84$ & 1.9 & 0.0226 & DOWN & 46967 \\
\hline $874.2963 / 16.28$ & 1.35 & 0.0226 & DOWN & NA \\
\hline $1010.6467 / 24.84$ & 2.12 & 0.0226 & DOWN & NA \\
\hline $549.3749 / 26.61$ & 1.66 & 0.0227 & DOWN & NA \\
\hline $1056.1426 / 24.84$ & 1.62 & 0.0227 & DOWN & 69813 \\
\hline $800.4834 / 24.84$ & 1.39 & 0.0227 & DOWN & NA \\
\hline $991.6735 / 24.84$ & 1.82 & 0.0227 & DOWN & NA \\
\hline $523.2992 / 24.84$ & 1.35 & 0.0228 & DOWN & 58161 \\
\hline $994.683 / 24.84$ & 1.77 & 0.0228 & DOWN & $\mathrm{NA}$ \\
\hline $1045.141 / 24.84$ & 1.8 & 0.0229 & DOWN & NA \\
\hline $259.6579 / 24.84$ & 1.39 & 0.0229 & DOWN & NA \\
\hline $502.2908 / 25.57$ & 1.75 & 0.023 & DOWN & 264379 \\
\hline $526.2932 / 23.03$ & 1.39 & 0.023 & DOWN & 62282 \\
\hline $1043.6357 / 24.84$ & 1.8 & 0.023 & DOWN & NA \\
\hline $1016.6648 / 24.84$ & 1.87 & 0.0231 & DOWN & NA \\
\hline $1057.1457 / 24.84$ & 1.5 & 0.0233 & DOWN & NA \\
\hline $670.3593 / 15.14$ & 1.57 & 0.0233 & DOWN & NA \\
\hline $521.6376 / 24.84$ & 1.34 & 0.0234 & DOWN & NA \\
\hline $467.1212 / 16.28$ & 1.25 & 0.0234 & DOWN & 987748 \\
\hline $280.6547 / 25.7$ & 1.4 & 0.0234 & DOWN & NA \\
\hline $992.6769 / 24.84$ & 1.83 & 0.0235 & DOWN & NA \\
\hline $772.4681 / 24.84$ & 1.63 & 0.0235 & DOWN & NA \\
\hline $600.2829 / 4.21$ & 2.08 & 0.0237 & UP & NA \\
\hline $281.1564 / 25.7$ & 1.37 & 0.0238 & DOWN & 985144 \\
\hline $455.2258 / 13.92$ & 1.29 & 0.0239 & UP & 154595 \\
\hline $523.8005 / 24.84$ & 1.34 & 0.024 & DOWN & $\mathrm{NA}$ \\
\hline $127.0389 / 16.28$ & 1.37 & 0.024 & DOWN & 35628 \\
\hline $1065.6012 / 24.84$ & 1.29 & 0.024 & DOWN & NA \\
\hline $1258.8155 / 24.84$ & 2.6 & 0.024 & DOWN & NA \\
\hline $1035.6387 / 24.84$ & 1.48 & 0.0241 & DOWN & NA \\
\hline $599.6146 / 4.21$ & 3.39 & 0.0241 & UP & NA \\
\hline $570.3433 / 14.59$ & 1.93 & 0.0243 & DOWN & 164973 \\
\hline $527.2968 / 23.03$ & 1.4 & 0.0243 & DOWN & NA \\
\hline $509.3446 / 24.1$ & 1.48 & 0.0243 & DOWN & NA \\
\hline $966.3982 / 16.74$ & 1.82 & 0.0243 & UP & NA \\
\hline
\end{tabular}




\begin{tabular}{|c|c|c|c|c|}
\hline $511.3596 / 26.13$ & 1.44 & 0.0243 & DOWN & NA \\
\hline $508.3404 / 24.1$ & 1.61 & 0.0244 & DOWN & 24068 \\
\hline $848.2783 / 16.28$ & 1.36 & 0.0245 & DOWN & NA \\
\hline $277.1358 / 24.84$ & 1.18 & 0.0245 & DOWN & NA \\
\hline $506.7571 / 6.5$ & 1.75 & 0.0246 & DOWN & NA \\
\hline $551.3152 / 25.7$ & 1.48 & 0.0249 & DOWN & NA \\
\hline $878.9968 / 28.09$ & 1.61 & 0.0249 & DOWN & NA \\
\hline $1003.6613 / 24.84$ & 2.06 & 0.025 & DOWN & NA \\
\hline $291.1512 / 28.08$ & 1.24 & 0.0251 & DOWN & NA \\
\hline $607.728 / 16.28$ & 1.66 & 0.0251 & DOWN & NA \\
\hline $482.2461 / 3.2$ & 1.97 & 0.0251 & DOWN & 43975 \\
\hline $1041.1267 / 24.84$ & 1.4 & 0.0251 & DOWN & NA \\
\hline $812.9431 / 24.84$ & 1.38 & 0.0251 & DOWN & NA \\
\hline $847.277 / 16.28$ & 1.37 & 0.0252 & DOWN & NA \\
\hline $473.1978 / 23.53$ & 1.58 & 0.0252 & DOWN & 44407 \\
\hline $791.9578 / 24.84$ & 1.26 & 0.0252 & DOWN & NA \\
\hline $104.107 / 25.7$ & 1.5 & 0.0252 & DOWN & $\mathrm{NA}$ \\
\hline $605.4241 / 27.88$ & 1.24 & 0.0252 & UP & 81214 \\
\hline $346.6264 / 16.28$ & 1.25 & 0.0253 & DOWN & NA \\
\hline $121.5097 / 4.18$ & 1.05 & 0.0253 & DOWN & $\mathrm{NA}$ \\
\hline $635.4016 / 24.84$ & 1.32 & 0.0254 & DOWN & 69744 \\
\hline $527.2988 / 24.84$ & 1.27 & 0.0256 & DOWN & 138026 \\
\hline $301.1412 / 23.57$ & 1.24 & 0.0256 & UP & 1060172 \\
\hline $589.2935 / 28.09$ & 1.26 & 0.0256 & DOWN & 63702 \\
\hline $792.9587 / 24.84$ & 1.43 & 0.0257 & DOWN & NA \\
\hline $542.3319 / 25.7$ & 1.56 & 0.0258 & DOWN & 162437 \\
\hline $438.2984 / 25.6$ & 1.63 & 0.0258 & DOWN & 1005933 \\
\hline $242.9347 / 0.77$ & 1.23 & 0.0259 & DOWN & NA \\
\hline $286.6366 / 24.84$ & 1.18 & 0.0259 & DOWN & NA \\
\hline $105.1103 / 24.84$ & 1.2 & 0.0259 & DOWN & NA \\
\hline $491.2339 / 16.28$ & 1.66 & 0.0259 & DOWN & NA \\
\hline $277.1535 / 24.84$ & 1.17 & 0.026 & DOWN & 1044503 \\
\hline $262.9067 / 0.69$ & 1.78 & 0.0261 & DOWN & 1065950 \\
\hline $172.9769 / 0.73$ & 1.11 & 0.0261 & UP & 2963 \\
\hline $469.1187 / 16.28$ & 1.3 & 0.0261 & DOWN & 995140 \\
\hline $1148.5549 / 24.84$ & 1.33 & 0.0261 & DOWN & $\mathrm{NA}$ \\
\hline $599.7417 / 16.28$ & 1.54 & 0.0261 & DOWN & $\mathrm{NA}$ \\
\hline $346.1247 / 16.28$ & 1.26 & 0.0261 & DOWN & 1032222 \\
\hline $111.0099 / 0.48$ & 1.15 & 0.0262 & DOWN & 2266 \\
\hline $504.344 / 25.73$ & 1.55 & 0.0262 & DOWN & 40378 \\
\hline $1018.6324 / 24.84$ & 1.96 & 0.0262 & DOWN & NA \\
\hline $407.1564 / 16.28$ & 1.25 & 0.0262 & DOWN & 1053751 \\
\hline $1259.3164 / 24.84$ & 2.42 & 0.0262 & DOWN & NA \\
\hline
\end{tabular}




\begin{tabular}{|c|c|c|c|c|}
\hline $412.1711 / 16.28$ & 1.32 & 0.0263 & DOWN & NA \\
\hline $588.3058 / 23.16$ & 1.36 & 0.0263 & UP & 188973 \\
\hline 438.1973/ 18.93 & 2.03 & 0.0263 & DOWN & NA \\
\hline $875.2982 / 16.28$ & 1.36 & 0.0263 & DOWN & NA \\
\hline $601.74 / 16.28$ & 1.47 & 0.0264 & DOWN & NA \\
\hline $217.9566 / 8.36$ & 1.06 & 0.0264 & UP & $\mathrm{NA}$ \\
\hline $118.0861 / 16.28$ & 1.43 & 0.0264 & DOWN & NA \\
\hline $1021.1347 / 24.84$ & 2 & 0.0264 & DOWN & NA \\
\hline $482.3248 / 23.17$ & 1.48 & 0.0265 & DOWN & 1086190 \\
\hline $830.2794 / 16.28$ & 1.36 & 0.0265 & DOWN & NA \\
\hline $1262.8256 / 24.84$ & 2.31 & 0.0266 & DOWN & NA \\
\hline $545.3415 / 25.7$ & 1.36 & 0.0266 & DOWN & NA \\
\hline $471.133 / 16.28$ & 1.17 & 0.0266 & DOWN & 65237 \\
\hline $794.3306 / 16.28$ & 1.78 & 0.0266 & DOWN & NA \\
\hline $240.0996 / 25.7$ & 1.41 & 0.0266 & DOWN & 58109 \\
\hline $794.8286 / 16.28$ & 1.62 & 0.0267 & DOWN & NA \\
\hline $1042.633 / 24.84$ & 1.73 & 0.0267 & DOWN & $\mathrm{NA}$ \\
\hline $281.6625 / 28.08$ & 1.28 & 0.0267 & DOWN & $\mathrm{NA}$ \\
\hline $408.1515 / 16.28$ & 1.27 & 0.0268 & DOWN & NA \\
\hline $504.3095 / 24.21$ & 1.26 & 0.0268 & DOWN & 62271 \\
\hline $289.1506 / 28.09$ & 1.47 & 0.0268 & DOWN & 22394 \\
\hline $726.2241 / 16.28$ & 1.43 & 0.0268 & DOWN & NA \\
\hline $267.2685 / 28.08$ & 1.45 & 0.0269 & DOWN & 6658 \\
\hline $565.2758 / 24.84$ & 1.15 & 0.0269 & DOWN & 213418 \\
\hline $427.1725 / 16.28$ & 1.27 & 0.0269 & DOWN & 19604 \\
\hline $1080.6022 / 24.84$ & 1.45 & 0.0269 & DOWN & NA \\
\hline $276.6519 / 24.84$ & 1.16 & 0.0269 & DOWN & NA \\
\hline $400.3427 / 22.74$ & 1.36 & 0.027 & DOWN & NA \\
\hline $599.2401 / 16.28$ & 1.53 & 0.027 & DOWN & 155757 \\
\hline $477.2783 / 24.71$ & 1.24 & 0.027 & DOWN & NA \\
\hline $1047.6468 / 24.84$ & 1.76 & 0.027 & DOWN & NA \\
\hline $98.9841 / 0.76$ & 1.66 & 0.0271 & DOWN & NA \\
\hline $1013.3776 / 16.28$ & 1.35 & 0.0272 & DOWN & $\mathrm{NA}$ \\
\hline $270.2794 / 29.48$ & 1.12 & 0.0272 & DOWN & NA \\
\hline $606.7289 / 16.28$ & 1.49 & 0.0273 & DOWN & NA \\
\hline $142.9481 / 0.67$ & 1.63 & 0.0273 & DOWN & NA \\
\hline $161.079 / 0.87$ & 1.52 & 0.0273 & UP & NA \\
\hline $496.5011 / 24.84$ & 1.56 & 0.0273 & DOWN & NA \\
\hline $165.0758 / 16.28$ & 1.43 & 0.0273 & DOWN & 95885 \\
\hline $1261.8238 / 24.84$ & 2.83 & 0.0274 & DOWN & 56134 \\
\hline $647.2817 / 28.09$ & 1.16 & 0.0274 & DOWN & NA \\
\hline $1019.6334 / 24.84$ & 2.04 & 0.0274 & DOWN & NA \\
\hline $549.279 / 23.51$ & 2.37 & 0.0274 & DOWN & NA \\
\hline
\end{tabular}




\begin{tabular}{|c|c|c|c|c|}
\hline $815.9523 / 24.84$ & 1.3 & 0.0274 & DOWN & NA \\
\hline $504.3065 / 23.17$ & 1.5 & 0.0274 & DOWN & 62271 \\
\hline $550.3155 / 25.7$ & 1.64 & 0.0275 & DOWN & 78847 \\
\hline $263.0916 / 16.28$ & 1.42 & 0.0275 & DOWN & 64561 \\
\hline $1020.634 / 24.84$ & 1.99 & 0.0275 & DOWN & NA \\
\hline $1020.1348 / 24.84$ & 2.03 & 0.0275 & DOWN & 64198 \\
\hline $602.2962 / 25.7$ & 1.41 & 0.0275 & DOWN & 264560 \\
\hline $247.0966 / 16.28$ & 1.45 & 0.0276 & DOWN & 64597 \\
\hline $533.3414 / 26.48$ & 1.51 & 0.0276 & DOWN & NA \\
\hline $1056.6444 / 24.84$ & 1.74 & 0.0276 & DOWN & NA \\
\hline $282.6653 / 28.08$ & 1.3 & 0.0277 & DOWN & NA \\
\hline $1261.3215 / 24.84$ & 2.74 & 0.0277 & DOWN & NA \\
\hline $560.2621 / 24.84$ & 1.27 & 0.0277 & DOWN & 188953 \\
\hline $1019.1344 / 24.84$ & 1.66 & 0.0278 & DOWN & NA \\
\hline $203.1068 / 16.28$ & 1.42 & 0.0278 & DOWN & 988142 \\
\hline $288.6701 / 29.65$ & 1.64 & 0.0278 & DOWN & NA \\
\hline $985.9152 / 16.28$ & 1.71 & 0.0278 & DOWN & 37981 \\
\hline $1036.6425 / 24.84$ & 1.42 & 0.0279 & DOWN & NA \\
\hline $241.9996 / 0.48$ & 1.22 & 0.028 & DOWN & NA \\
\hline $523.3595 / 25.7$ & 1.38 & 0.028 & DOWN & NA \\
\hline $1312.2892 / 24.84$ & 1.6 & 0.028 & DOWN & NA \\
\hline $1165.4396 / 16.71$ & 1.95 & 0.0281 & UP & NA \\
\hline $145.0495 / 16.28$ & 1.41 & 0.0281 & DOWN & 44698 \\
\hline $626.7008 / 16.28$ & 1.27 & 0.0281 & DOWN & NA \\
\hline $808.9796 / 24.84$ & 1.32 & 0.0281 & DOWN & $\mathrm{NA}$ \\
\hline $600.2431 / 16.28$ & 1.53 & 0.0282 & DOWN & 264248 \\
\hline $522.3054 / 24.84$ & 1.39 & 0.0283 & DOWN & NA \\
\hline $793.33 / 16.28$ & 1.78 & 0.0283 & DOWN & $\mathrm{NA}$ \\
\hline $500.2752 / 23.54$ & 2.56 & 0.0283 & DOWN & 130433 \\
\hline $1303.8072 / 24.84$ & 1.68 & 0.0283 & DOWN & NA \\
\hline $1259.8172 / 24.84$ & 2.06 & 0.0284 & DOWN & NA \\
\hline $524.3624 / 25.7$ & 1.39 & 0.0285 & DOWN & NA \\
\hline $406.1535 / 16.28$ & 1.23 & 0.0286 & DOWN & 994994 \\
\hline $1271.5687 / 16.74$ & 2.09 & 0.0286 & UP & 90717 \\
\hline $833.4388 / 24.84$ & 1.26 & 0.0286 & DOWN & NA \\
\hline $607.2266 / 16.28$ & 1.63 & 0.0287 & DOWN & 114058 \\
\hline $153.0383 / 16.28$ & 1.31 & 0.0288 & DOWN & 88838 \\
\hline $122.0681 / 16.28$ & 1.34 & 0.0288 & DOWN & NA \\
\hline $541.8307 / 25.7$ & 1.68 & 0.0289 & DOWN & NA \\
\hline $1090.5935 / 24.84$ & 1.39 & 0.029 & DOWN & NA \\
\hline $837.7725 / 16.28$ & 1.33 & 0.029 & DOWN & NA \\
\hline $311.1622 / 28.08$ & 1.26 & 0.029 & DOWN & 986940 \\
\hline $1076.6269 / 24.84$ & 1.36 & 0.0291 & DOWN & NA \\
\hline
\end{tabular}




\begin{tabular}{|c|c|c|c|c|}
\hline $572.3715 / 26.17$ & 1.43 & 0.0291 & DOWN & NA \\
\hline $799.9804 / 24.84$ & 1.3 & 0.0292 & DOWN & NA \\
\hline $814.3103 / 16.28$ & 1.37 & 0.0292 & DOWN & NA \\
\hline $105.0698 / 16.28$ & 1.39 & 0.0292 & DOWN & NA \\
\hline $600.7444 / 16.28$ & 1.51 & 0.0293 & DOWN & NA \\
\hline $805.9674 / 24.84$ & 1.3 & 0.0293 & DOWN & NA \\
\hline $110.0086 / 0.48$ & 1.14 & 0.0293 & DOWN & NA \\
\hline $437.1938 / 18.93$ & 2.01 & 0.0295 & DOWN & 23595 \\
\hline $369.17 / 16.28$ & 1.52 & 0.0296 & DOWN & 987170 \\
\hline $964.8807 / 16.69$ & 1.65 & 0.0296 & UP & NA \\
\hline $482.3247 / 27.97$ & 1.37 & 0.0296 & DOWN & 1086190 \\
\hline $792.3267 / 16.28$ & 1.76 & 0.0297 & DOWN & NA \\
\hline $986.4163 / 16.28$ & 1.58 & 0.0298 & DOWN & NA \\
\hline $324.9392 / 0.75$ & 1.72 & 0.0298 & DOWN & 1046727 \\
\hline $564.2405 / 23.51$ & 2.21 & 0.03 & DOWN & 57984 \\
\hline $785.8367 / 16.28$ & 1.52 & 0.03 & DOWN & NA \\
\hline $792.8283 / 16.28$ & 1.76 & 0.03 & DOWN & NA \\
\hline $1216.1539 / 16.69$ & 2.07 & 0.0301 & UP & NA \\
\hline $1069.5992 / 24.84$ & 1.38 & 0.0302 & DOWN & NA \\
\hline $522.356 / 25.7$ & 1.38 & 0.0302 & DOWN & 34488 \\
\hline $490.2312 / 16.28$ & 1.63 & 0.0302 & DOWN & NA \\
\hline $1066.6903 / 25.7$ & 1.51 & 0.0302 & DOWN & NA \\
\hline $383.1494 / 16.28$ & 1.66 & 0.0303 & DOWN & 1034601 \\
\hline $863.2493 / 16.28$ & 1.38 & 0.0303 & DOWN & 3696 \\
\hline $472.1364 / 16.28$ & 1.19 & 0.0304 & DOWN & NA \\
\hline $548.2754 / 23.51$ & 2.64 & 0.0304 & DOWN & 264685 \\
\hline $1295.8159 / 24.84$ & 1.96 & 0.0305 & DOWN & NA \\
\hline $793.8315 / 16.28$ & 1.76 & 0.0306 & DOWN & NA \\
\hline $812.3106 / 16.28$ & 1.23 & 0.0306 & DOWN & NA \\
\hline $541.3295 / 25.7$ & 1.71 & 0.0306 & DOWN & 89990 \\
\hline $1247.6077 / 16.45$ & 1.36 & 0.0306 & DOWN & NA \\
\hline $527.2967 / 23.51$ & 2.58 & 0.0306 & DOWN & NA \\
\hline $483.3282 / 23.17$ & 1.51 & 0.0307 & DOWN & NA \\
\hline $339.2899 / 24.94$ & 1.89 & 0.0307 & DOWN & 73128 \\
\hline $521.3313 / 24.84$ & 1.31 & 0.0308 & DOWN & NA \\
\hline $1003.162 / 24.84$ & 2.02 & 0.0309 & DOWN & NA \\
\hline $1110.5862 / 24.84$ & 1.36 & 0.0309 & DOWN & NA \\
\hline $543.0628 / 16.3$ & 1.27 & 0.0311 & UP & NA \\
\hline $263.0282 / 4.18$ & 1.06 & 0.0311 & DOWN & 72834 \\
\hline $355.6126 / 16.28$ & 1.19 & 0.0311 & DOWN & NA \\
\hline $339.9039 / 0.76$ & 1.58 & 0.0314 & DOWN & NA \\
\hline $601.2413 / 16.28$ & 1.52 & 0.0314 & DOWN & 124658 \\
\hline $1301.3051 / 24.84$ & 1.73 & 0.0314 & DOWN & NA \\
\hline
\end{tabular}




\begin{tabular}{|c|c|c|c|c|}
\hline $1331.7824 / 24.84$ & 1.61 & 0.0314 & DOWN & NA \\
\hline $858.3093 / 16.28$ & 1.38 & 0.0315 & DOWN & NA \\
\hline $765.3792 / 5.21$ & 2.11 & 0.0316 & DOWN & 264419 \\
\hline $250.1157 / 16.28$ & 1.6 & 0.0318 & DOWN & NA \\
\hline $249.1123 / 16.28$ & 1.57 & 0.0318 & DOWN & 44826 \\
\hline $526.2934 / 23.51$ & 2.62 & 0.0318 & DOWN & 62282 \\
\hline $406.6552 / 16.28$ & 1.24 & 0.0319 & DOWN & NA \\
\hline $1213.4424 / 16.28$ & 1.45 & 0.032 & DOWN & NA \\
\hline $294.0819 / 16.28$ & 1.25 & 0.0321 & DOWN & 1063969 \\
\hline $1050.1141 / 24.84$ & 1.39 & 0.0321 & DOWN & NA \\
\hline $492.2371 / 16.28$ & 1.54 & 0.0321 & DOWN & NA \\
\hline $1074.6137 / 24.84$ & 1.41 & 0.0321 & DOWN & NA \\
\hline $571.3049 / 28.08$ & 1.33 & 0.0321 & DOWN & 196973 \\
\hline $129.0545 / 16.28$ & 1.36 & 0.0322 & DOWN & 35704 \\
\hline $385.1683 / 29.68$ & 1.45 & 0.0323 & DOWN & 986328 \\
\hline $725.222 / 16.28$ & 1.45 & 0.0323 & DOWN & NA \\
\hline $407.6574 / 16.28$ & 1.29 & 0.0323 & DOWN & $\mathrm{NA}$ \\
\hline $489.2276 / 16.28$ & 1.61 & 0.0324 & DOWN & 47814 \\
\hline $281.1023 / 16.28$ & 1.31 & 0.0324 & DOWN & 3377 \\
\hline $478.2934 / 23.54$ & 2.82 & 0.0325 & DOWN & 40778 \\
\hline $1110.3917 / 16.28$ & 1.36 & 0.0325 & DOWN & NA \\
\hline $156.0142 / 0.48$ & 1.16 & 0.0325 & DOWN & NA \\
\hline $593.8103 / 28.08$ & 1.32 & 0.0326 & DOWN & NA \\
\hline $1292.3063 / 24.84$ & 1.68 & 0.0327 & DOWN & NA \\
\hline $282.1055 / 16.28$ & 1.35 & 0.0327 & DOWN & NA \\
\hline $298.6465 / 25.7$ & 1.33 & 0.0328 & DOWN & NA \\
\hline $795.3354 / 16.28$ & 1.41 & 0.0328 & DOWN & NA \\
\hline $479.3333 / 24.84$ & 1.28 & 0.0328 & DOWN & NA \\
\hline $1258.3126 / 24.84$ & 2.35 & 0.0329 & DOWN & NA \\
\hline $504.3066 / 27.96$ & 1.35 & 0.033 & DOWN & 62271 \\
\hline $1072.6146 / 24.84$ & 1.31 & 0.033 & DOWN & NA \\
\hline $501.2788 / 23.54$ & 2.3 & 0.0332 & DOWN & NA \\
\hline $1002.6611 / 24.84$ & 1.68 & 0.0332 & DOWN & NA \\
\hline $1262.3247 / 24.84$ & 2.52 & 0.0332 & DOWN & NA \\
\hline $399.0876 / 0.59$ & 1.17 & 0.0332 & UP & 50066 \\
\hline $460.2829 / 23.54$ & 2.55 & 0.0334 & DOWN & 10090909 \\
\hline $1011.8691 / 16.28$ & 1.49 & 0.0334 & DOWN & NA \\
\hline $1137.7035 / 28.09$ & 1.64 & 0.0334 & DOWN & NA \\
\hline $811.3077 / 16.28$ & 1.25 & 0.0335 & DOWN & 44896 \\
\hline $590.2926 / 28.08$ & 1.25 & 0.0336 & DOWN & NA \\
\hline $258.6235 / 23.53$ & 2.62 & 0.0337 & DOWN & NA \\
\hline $221.0532 / 16.28$ & 1.19 & 0.0338 & DOWN & 1049967 \\
\hline $525.2788 / 23.58$ & 1.76 & 0.0342 & DOWN & NA \\
\hline
\end{tabular}




\begin{tabular}{|c|c|c|c|c|}
\hline $561.7631 / 24.84$ & 1.17 & 0.0345 & DOWN & NA \\
\hline $174.9915 / 0.78$ & 1.22 & 0.0345 & UP & 71607 \\
\hline $316.9941 / 0.76$ & 1.27 & 0.0347 & DOWN & 1075643 \\
\hline $500.2776 / 21.37$ & 1.32 & 0.0347 & DOWN & 680632 \\
\hline $451.2307 / 15.11$ & 1.17 & 0.0348 & DOWN & 44903 \\
\hline $1304.3135 / 24.84$ & 1.73 & 0.0348 & DOWN & NA \\
\hline $571.3465 / 14.6$ & 1.57 & 0.0349 & DOWN & NA \\
\hline $351.2296 / 29.68$ & 1.62 & 0.0349 & DOWN & 1034019 \\
\hline $1050.6126 / 24.84$ & 1.4 & 0.035 & DOWN & NA \\
\hline $479.2968 / 23.53$ & 2.76 & 0.035 & DOWN & NA \\
\hline $1060.6085 / 24.84$ & 1.44 & 0.035 & DOWN & NA \\
\hline $104.1069 / 28.08$ & 1.21 & 0.035 & DOWN & NA \\
\hline $827.2702 / 16.28$ & 1.32 & 0.0351 & DOWN & NA \\
\hline $291.6472 / 28.09$ & 1.25 & 0.0354 & DOWN & NA \\
\hline $570.3539 / 26.6$ & 1.65 & 0.0354 & DOWN & 61710 \\
\hline $321.0414 / 16.28$ & 1.3 & 0.0354 & DOWN & 89369 \\
\hline $803.3143 / 16.28$ & 1.6 & 0.0355 & DOWN & 43668 \\
\hline $1039.6267 / 24.84$ & 1.58 & 0.0355 & DOWN & NA \\
\hline $835.9472 / 24.84$ & 1.19 & 0.0356 & DOWN & NA \\
\hline $1040.6262 / 24.84$ & 1.52 & 0.0356 & DOWN & $\mathrm{NA}$ \\
\hline $306.1518 / 24.84$ & 1.13 & 0.0356 & DOWN & 96662 \\
\hline $532.338 / 26.48$ & 1.39 & 0.0357 & DOWN & 62280 \\
\hline $604.3117 / 28.09$ & 1.1 & 0.0358 & DOWN & 264553 \\
\hline $282.1643 / 28.08$ & 1.26 & 0.0358 & DOWN & 985850 \\
\hline $427.1369 / 16.28$ & 1.24 & 0.0359 & DOWN & NA \\
\hline $521.9713 / 24.84$ & 1.36 & 0.036 & DOWN & NA \\
\hline $1287.294 / 24.84$ & 1.53 & 0.036 & DOWN & 47038 \\
\hline $1094.6057 / 24.84$ & 1.32 & 0.0361 & DOWN & $\mathrm{NA}$ \\
\hline $1271.4322 / 16.72$ & 1.84 & 0.0362 & UP & NA \\
\hline $796.3388 / 16.28$ & 1.38 & 0.0362 & DOWN & NA \\
\hline $314.03 / 28.09$ & 1.26 & 0.0363 & DOWN & 1073886 \\
\hline $964.8452 / 16.69$ & 1.56 & 0.0363 & UP & NA \\
\hline $867.248 / 16.28$ & 1.3 & 0.0363 & DOWN & NA \\
\hline $596.2794 / 25.7$ & 1.46 & 0.0364 & DOWN & 264213 \\
\hline $838.2736 / 16.28$ & 1.41 & 0.0364 & DOWN & NA \\
\hline $857.3063 / 16.28$ & 1.35 & 0.0365 & DOWN & NA \\
\hline $1043.7043 / 25.7$ & 1.69 & 0.0365 & DOWN & NA \\
\hline $813.9455 / 24.84$ & 1.21 & 0.0366 & DOWN & NA \\
\hline $370.1736 / 16.28$ & 1.49 & 0.0367 & DOWN & NA \\
\hline $822.4334 / 24.84$ & 1.25 & 0.0368 & DOWN & NA \\
\hline $1332.7855 / 24.84$ & 1.46 & 0.0368 & DOWN & NA \\
\hline $289.6417 / 25.7$ & 1.33 & 0.0368 & DOWN & NA \\
\hline $193.9299 / 0.76$ & 1.17 & 0.0368 & DOWN & NA \\
\hline
\end{tabular}




\begin{tabular}{|c|c|c|c|c|}
\hline $544.3381 / 25.7$ & 1.35 & 0.0369 & DOWN & 61703 \\
\hline $1065.6867 / 25.7$ & 1.58 & 0.0369 & DOWN & NA \\
\hline $1068.6016 / 24.84$ & 1.44 & 0.0373 & DOWN & NA \\
\hline $865.2488 / 16.28$ & 1.34 & 0.0373 & DOWN & NA \\
\hline $511.3956 / 28.99$ & 1.46 & 0.0376 & DOWN & NA \\
\hline $724.2178 / 16.28$ & 1.45 & 0.0377 & DOWN & NA \\
\hline $235.1695 / 21.44$ & 1.05 & 0.0379 & DOWN & 985513 \\
\hline $797.3418 / 16.28$ & 1.37 & 0.038 & DOWN & $\mathrm{NA}$ \\
\hline $874.5806 / 16.74$ & 1.66 & 0.0382 & UP & NA \\
\hline $926.2973 / 16.28$ & 1.31 & 0.0383 & DOWN & NA \\
\hline $539.3908 / 29.65$ & 1.44 & 0.0384 & DOWN & NA \\
\hline $144.102 / 0.63$ & 1.34 & 0.0384 & DOWN & 35932 \\
\hline $1287.795 / 24.84$ & 1.73 & 0.0384 & DOWN & NA \\
\hline $1075.696 / 28.08$ & 1.63 & 0.0385 & DOWN & NA \\
\hline $260.639 / 27.97$ & 1.4 & 0.0385 & DOWN & NA \\
\hline $1062.1177 / 24.84$ & 1.42 & 0.0385 & DOWN & NA \\
\hline $524.3717 / 28.08$ & 1.34 & 0.0385 & DOWN & NA \\
\hline $836.7704 / 16.28$ & 1.36 & 0.0386 & DOWN & NA \\
\hline $798.3447 / 16.28$ & 1.4 & 0.0387 & DOWN & NA \\
\hline $119.0491 / 16.28$ & 1.44 & 0.0388 & DOWN & 7012 \\
\hline $1084.6155 / 24.84$ & 1.24 & 0.039 & DOWN & $\mathrm{NA}$ \\
\hline $317.6167 / 25.69$ & 1.3 & 0.039 & DOWN & NA \\
\hline $297.1466 / 24.84$ & 1.15 & 0.0391 & DOWN & 985809 \\
\hline $770.4641 / 24.84$ & 1.35 & 0.0391 & DOWN & NA \\
\hline $609.2267 / 16.28$ & 1.46 & 0.0392 & DOWN & NA \\
\hline $282.6233 / 23.02$ & 1.31 & 0.0395 & DOWN & NA \\
\hline $608.2272 / 16.28$ & 1.48 & 0.0395 & DOWN & NA \\
\hline $483.3283 / 27.96$ & 1.31 & 0.0395 & DOWN & NA \\
\hline $1054.1333 / 24.84$ & 1.52 & 0.0395 & DOWN & NA \\
\hline $574.2624 / 24.84$ & 1.11 & 0.0397 & DOWN & NA \\
\hline $750.3164 / 28.09$ & 1.21 & 0.0398 & DOWN & 991236 \\
\hline $528.2993 / 23.02$ & 1.24 & 0.0398 & DOWN & NA \\
\hline $540.3057 / 24.84$ & 1.19 & 0.0398 & DOWN & 265004 \\
\hline $338.0873 / 2.89$ & 2.16 & 0.0398 & DOWN & 145590 \\
\hline $1247.4645 / 16.45$ & 1.36 & 0.0399 & DOWN & NA \\
\hline $480.2994 / 23.53$ & 2.25 & 0.04 & DOWN & NA \\
\hline $526.3779 / 28.08$ & 1.31 & 0.0401 & DOWN & NA \\
\hline 985.4127/ 16.28 & 1.58 & 0.0402 & DOWN & 84999 \\
\hline $415.2121 / 18.93$ & 1.87 & 0.0403 & DOWN & 985282 \\
\hline $1247.7505 / 16.46$ & 1.47 & 0.0405 & DOWN & NA \\
\hline $800.3128 / 16.28$ & 1.69 & 0.0405 & DOWN & 3660 \\
\hline $260.639 / 23.17$ & 1.43 & 0.0407 & DOWN & $\mathrm{NA}$ \\
\hline $802.3144 / 16.28$ & 1.65 & 0.0408 & DOWN & NA \\
\hline
\end{tabular}




\begin{tabular}{|c|c|c|c|c|}
\hline $608.3242 / 28.09$ & 1.14 & 0.0408 & DOWN & NA \\
\hline $528.2991 / 23.51$ & 2.18 & 0.0409 & DOWN & NA \\
\hline $298.145 / 24.84$ & 1.13 & 0.041 & DOWN & NA \\
\hline $1140.1777 / 28.09$ & 1.43 & 0.0411 & DOWN & NA \\
\hline $278.639 / 21.91$ & 1.75 & 0.0411 & DOWN & NA \\
\hline $246.9916 / 4.18$ & 1.04 & 0.0411 & DOWN & 44557 \\
\hline $608.7284 / 16.28$ & 1.47 & 0.0414 & DOWN & NA \\
\hline $803.8143 / 16.28$ & 1.46 & 0.0414 & DOWN & NA \\
\hline 932.6848/16.69 & 1.68 & 0.0414 & UP & 39849 \\
\hline $621.2818 / 23.66$ & 1.16 & 0.0416 & DOWN & 81194 \\
\hline $841.9229 / 24.84$ & 1.27 & 0.0417 & DOWN & NA \\
\hline $818.7821 / 16.28$ & 1.36 & 0.0418 & DOWN & NA \\
\hline $516.2408 / 23.53$ & 1.94 & 0.0419 & DOWN & 1019038 \\
\hline $108.5125 / 0.83$ & 1.28 & 0.0421 & UP & NA \\
\hline $106.0732 / 16.28$ & 1.34 & 0.0424 & DOWN & $\mathrm{NA}$ \\
\hline $414.14 / 16.28$ & 1.29 & 0.0424 & DOWN & 1022692 \\
\hline $767.493 / 24.84$ & 1.49 & 0.0425 & DOWN & NA \\
\hline $290.1431 / 25.7$ & 1.44 & 0.0425 & DOWN & NA \\
\hline $387.1804 / 16.28$ & 1.3 & 0.0425 & DOWN & 1028507 \\
\hline $128.0192 / 0.49$ & 1.11 & 0.0425 & DOWN & NA \\
\hline 964.7438/16.7 & 1.66 & 0.0426 & UP & 40484 \\
\hline $290.6498 / 28.08$ & 1.22 & 0.0426 & DOWN & NA \\
\hline $1311.7877 / 24.84$ & 1.58 & 0.0426 & DOWN & NA \\
\hline $130.0499 / 0.6$ & 1.21 & 0.0427 & DOWN & 22 \\
\hline $519.3282 / 21.91$ & 1.48 & 0.0428 & DOWN & NA \\
\hline $874.5494 / 16.7$ & 1.88 & 0.0428 & UP & NA \\
\hline $801.314 / 16.28$ & 1.66 & 0.0428 & DOWN & NA \\
\hline $808.5374 / 28.08$ & 1.72 & 0.0429 & DOWN & 46679 \\
\hline $1247.8939 / 16.45$ & 1.42 & 0.0429 & DOWN & NA \\
\hline $819.2844 / 16.28$ & 1.35 & 0.043 & DOWN & 58459 \\
\hline $524.2753 / 23.04$ & 1.37 & 0.043 & DOWN & 5554 \\
\hline $561.3976 / 27.82$ & 1.23 & 0.043 & DOWN & 5976 \\
\hline $580.2938 / 28.09$ & 1.18 & 0.0431 & DOWN & 163778 \\
\hline $213.5687 / 16.28$ & 1.22 & 0.0431 & DOWN & NA \\
\hline $527.3806 / 28.08$ & 1.35 & 0.0432 & DOWN & NA \\
\hline $543.8464 / 28.08$ & 1.46 & 0.0432 & DOWN & NA \\
\hline $680.2534 / 28.09$ & 1.19 & 0.0433 & DOWN & 43770 \\
\hline $234.9081 / 0.75$ & 1.51 & 0.0434 & DOWN & NA \\
\hline $684.2557 / 25.7$ & 1.26 & 0.0434 & DOWN & 264597 \\
\hline $547.3572 / 28.08$ & 1.28 & 0.0437 & DOWN & NA \\
\hline $525.3751 / 28.08$ & 1.32 & 0.0437 & DOWN & NA \\
\hline $151.0352 / 0.48$ & 1.11 & 0.0437 & DOWN & 88435 \\
\hline $506.3612 / 28.08$ & 1.43 & 0.0438 & DOWN & 40373 \\
\hline
\end{tabular}




\begin{tabular}{|c|c|c|c|c|}
\hline $455.2966 / 24.71$ & 1.19 & 0.0438 & DOWN & NA \\
\hline $783.9589 / 24.84$ & 1.31 & 0.0439 & DOWN & NA \\
\hline $406.2133 / 16.28$ & 1.36 & 0.0439 & DOWN & NA \\
\hline $1036.0574 / 16.72$ & 1.92 & 0.0439 & UP & NA \\
\hline $198.0852 / 0.58$ & 1.29 & 0.044 & DOWN & NA \\
\hline $552.8327 / 28.08$ & 1.43 & 0.044 & DOWN & NA \\
\hline $819.7865 / 16.28$ & 1.37 & 0.0441 & DOWN & NA \\
\hline $275.133 / 24.84$ & 1.21 & 0.0441 & DOWN & 985919 \\
\hline $1120.1821 / 28.08$ & 1.64 & 0.0443 & DOWN & NA \\
\hline $544.849 / 28.09$ & 1.43 & 0.0443 & DOWN & NA \\
\hline $409.1624 / 16.28$ & 1.29 & 0.0444 & DOWN & 1053790 \\
\hline $320.0344 / 16.28$ & 1.24 & 0.0444 & DOWN & 1048495 \\
\hline $1034.188 / 15.65$ & 1.51 & 0.0444 & UP & $\mathrm{NA}$ \\
\hline $502.2933 / 23.58$ & 2.34 & 0.0445 & DOWN & 62272 \\
\hline $815.0137 / 28.08$ & 1.69 & 0.0446 & DOWN & NA \\
\hline $160.0757 / 0.87$ & 1.44 & 0.0448 & UP & 6932 \\
\hline $282.6231 / 23.51$ & 1.81 & 0.0449 & DOWN & NA \\
\hline $932.5864 / 16.7$ & 1.59 & 0.045 & UP & NA \\
\hline $534.2927 / 24.84$ & 1.24 & 0.045 & DOWN & 129228 \\
\hline $806.0306 / 28.09$ & 1.64 & 0.045 & DOWN & NA \\
\hline $1296.3189 / 24.84$ & 1.39 & 0.0452 & DOWN & NA \\
\hline $411.1685 / 16.28$ & 1.27 & 0.0452 & DOWN & NA \\
\hline $302.3058 / 18.73$ & 1.21 & 0.0453 & DOWN & NA \\
\hline $526.3002 / 24.84$ & 1.22 & 0.0453 & DOWN & NA \\
\hline $801.8148 / 16.28$ & 1.65 & 0.0453 & DOWN & $\mathrm{NA}$ \\
\hline $880.3084 / 16.28$ & 1.27 & 0.0453 & DOWN & 991880 \\
\hline $838.0157 / 28.08$ & 1.35 & 0.0456 & DOWN & NA \\
\hline $1305.2006 / 16.28$ & 1.55 & 0.0457 & UP & NA \\
\hline $289.6594 / 25.69$ & 1.27 & 0.0458 & DOWN & NA \\
\hline $410.1658 / 16.28$ & 1.29 & 0.0459 & DOWN & NA \\
\hline $1131.6869 / 28.08$ & 1.38 & 0.0459 & DOWN & NA \\
\hline $99.512 / 24.59$ & 1.38 & 0.0459 & UP & NA \\
\hline $437.2855 / 24.7$ & 1.46 & 0.046 & DOWN & NA \\
\hline $1236.8507 / 16.27$ & 1.46 & 0.046 & UP & NA \\
\hline $524.2751 / 23.58$ & 2.14 & 0.046 & DOWN & 5554 \\
\hline $1249.6073 / 16.46$ & 1.24 & 0.0461 & DOWN & $\mathrm{NA}$ \\
\hline $543.3448 / 28.08$ & 1.45 & 0.0461 & DOWN & 84015 \\
\hline $525.3644 / 25.7$ & 1.31 & 0.0462 & DOWN & NA \\
\hline $790.3802 / 16.28$ & 1.35 & 0.0462 & DOWN & NA \\
\hline $1170.5447 / 24.84$ & 1.34 & 0.0463 & DOWN & NA \\
\hline $311.6634 / 28.09$ & 1.2 & 0.0463 & DOWN & NA \\
\hline $1036.3151 / 16.73$ & 1.75 & 0.0465 & UP & NA \\
\hline $815.5139 / 28.08$ & 1.44 & 0.0465 & DOWN & NA \\
\hline
\end{tabular}




\begin{tabular}{|c|c|c|c|c|}
\hline $1049.613 / 24.84$ & 1.29 & 0.0465 & DOWN & NA \\
\hline $404.2071 / 16.28$ & 1.35 & 0.0466 & DOWN & 67124 \\
\hline $853.2937 / 16.28$ & 1.25 & 0.0466 & DOWN & NA \\
\hline $299.6543 / 28.09$ & 1.21 & 0.0467 & DOWN & NA \\
\hline $829.276 / 16.28$ & 1.32 & 0.0467 & DOWN & NA \\
\hline $1018.1294 / 24.84$ & 1.64 & 0.0468 & DOWN & NA \\
\hline $845.5176 / 15.65$ & 1.79 & 0.0468 & UP & 80143 \\
\hline $1029.6266 / 24.84$ & 1.48 & 0.0469 & DOWN & NA \\
\hline $304.6491 / 23.13$ & 1.2 & 0.0469 & UP & NA \\
\hline $269.1291 / 16.28$ & 1.41 & 0.0469 & DOWN & NA \\
\hline $1033.9659 / 15.65$ & 1.69 & 0.0471 & UP & NA \\
\hline $544.3478 / 28.08$ & 1.44 & 0.0471 & DOWN & NA \\
\hline $995.6871 / 24.84$ & 1.44 & 0.0472 & DOWN & NA \\
\hline $123.1169 / 2.05$ & 1.38 & 0.0474 & DOWN & 87666 \\
\hline $598.2623 / 23.68$ & 1.26 & 0.0474 & DOWN & 121379 \\
\hline $375.5486 / 28.09$ & 1.27 & 0.0475 & DOWN & NA \\
\hline $1165.7293 / 16.69$ & 1.84 & 0.0476 & UP & NA \\
\hline $791.3837 / 16.28$ & 1.36 & 0.0476 & DOWN & NA \\
\hline $1096.2744 / 16.15$ & 1.86 & 0.0477 & UP & 90746 \\
\hline $1067.7121 / 28.07$ & 1.77 & 0.0477 & DOWN & NA \\
\hline $119.0856 / 18.93$ & 1.95 & 0.0477 & DOWN & 70027 \\
\hline $855.4906 / 28.08$ & 1.41 & 0.0477 & DOWN & NA \\
\hline $517.8125 / 24.84$ & 1.26 & 0.0478 & DOWN & NA \\
\hline $609.7268 / 16.28$ & 1.44 & 0.0478 & DOWN & NA \\
\hline $795.9667 / 24.84$ & 1.2 & 0.0478 & DOWN & NA \\
\hline $289.6491 / 28.09$ & 1.23 & 0.0479 & DOWN & NA \\
\hline $1050.7449 / 28.08$ & 1.61 & 0.0479 & DOWN & NA \\
\hline $559.7584 / 24.84$ & 1.32 & 0.0479 & DOWN & NA \\
\hline $503.2969 / 23.58$ & 2.29 & 0.048 & DOWN & NA \\
\hline $384.1527 / 16.28$ & 1.48 & 0.048 & DOWN & NA \\
\hline $847.8981 / 16.71$ & 1.61 & 0.048 & UP & NA \\
\hline $1288.7943 / 24.84$ & 1.43 & 0.048 & DOWN & NA \\
\hline $147.0652 / 16.28$ & 1.34 & 0.0481 & DOWN & 44745 \\
\hline $409.1624 / 15.81$ & 1.32 & 0.0481 & DOWN & 1053790 \\
\hline $416.139 / 16.28$ & 1.17 & 0.0481 & DOWN & NA \\
\hline $267.6096 / 23.54$ & 1.62 & 0.0481 & DOWN & NA \\
\hline $259.6312 / 25.58$ & 1.45 & 0.0482 & DOWN & NA \\
\hline $546.3537 / 28.08$ & 1.26 & 0.0482 & DOWN & 40019 \\
\hline $118.0862 / 0.61$ & 1.36 & 0.0482 & DOWN & 92812 \\
\hline $777.435 / 16.74$ & 1.89 & 0.0482 & UP & NA \\
\hline $1127.6759 / 28.08$ & 1.36 & 0.0482 & DOWN & NA \\
\hline $964.9838 / 16.68$ & 1.71 & 0.0483 & UP & NA \\
\hline $361.2741 / 23.04$ & 1.41 & 0.0483 & DOWN & 43158 \\
\hline
\end{tabular}




\begin{tabular}{|l|l|l|l|l|}
\hline $814.5145 / 28.09$ & 1.61 & 0.0484 & DOWN & NA \\
\hline $293.5786 / 16.28$ & 1.21 & 0.0484 & DOWN & NA \\
\hline $240.0997 / 28.09$ & 1.28 & 0.0484 & DOWN & 58109 \\
\hline $183.5352 / 16.27$ & 1.32 & 0.0485 & DOWN & NA \\
\hline $425.1367 / 14.95$ & 1.68 & 0.0485 & DOWN & 1090334 \\
\hline $571.3573 / 26.61$ & 1.31 & 0.0485 & DOWN & NA \\
\hline $1091.4072 / 16.45$ & 1.43 & 0.0486 & DOWN & NA \\
\hline $354.1109 / 16.28$ & 1.22 & 0.0486 & DOWN & 1041653 \\
\hline $809.5411 / 28.08$ & 1.34 & 0.0486 & DOWN & NA \\
\hline $405.2105 / 16.28$ & 1.34 & 0.0487 & DOWN & NA \\
\hline $512.3622 / 26.48$ & 1.31 & 0.0487 & DOWN & NA \\
\hline $805.0268 / 28.09$ & 1.76 & 0.0488 & DOWN & NA \\
\hline $578.2764 / 24.85$ & 1.29 & 0.0489 & DOWN & NA \\
\hline $295.0824 / 16.28$ & 1.16 & 0.0489 & DOWN & NA \\
\hline $1271.5216 / 16.73$ & 1.71 & 0.0491 & UP & NA \\
\hline $337.2741 / 22.92$ & 1.94 & 0.0492 & DOWN & 73127 \\
\hline $500.2779 / 21.9$ & 2 & 0.0493 & DOWN & 680632 \\
\hline $329.2479 / 29.67$ & 1.52 & 0.0494 & DOWN & 34850 \\
\hline $267.6098 / 22.92$ & 1.46 & 0.0494 & DOWN & NA \\
\hline $553.8314 / 28.08$ & 1.42 & 0.0495 & DOWN & NA \\
\hline $1012.8722 / 16.28$ & 1.45 & 0.0495 & DOWN & 39940 \\
\hline $930.2396 / 16.28$ & 1.18 & 0.0496 & DOWN & NA \\
\hline $251.0004 / 16.43$ & 1.27 & 0.0497 & UP & 73162 \\
\hline $239.2374 / 24.84$ & 1.16 & 0.0497 & DOWN & 36487 \\
\hline $516.2401 / 22.92$ & 1.72 & 0.0498 & DOWN & 1019038 \\
\hline & & & \\
\hline
\end{tabular}

NA: No associated METLIN ID 


\section{CHAPTER IV}

\section{The Gut Microbiota Modulates Differential Adenoma Suppression by}

\section{B6/J and B6/N Genetic Backgrounds in Min mice}

(Jacob Moskowitz, Federica Andreatta, and James Amos-Landgraf) 


\subsection{Overview}

The challenges of controlling for genetic and environmental variability in the study of human diseases such as colorectal cancer (CRC) necessitate the use of animal models that enable the resolution of complex traits. The $A p c^{\operatorname{Min}}$ (Min) mouse model of CRC, which harbors an autosomal dominant mutation in the Apc tumor suppressor gene causing the development of intestinal adenomas, provides an extensively studied and quantitative platform to investigate factors contributing to disease initiation (Shoemaker, Gould et al. 1997). Due to the well-known phenotypic variability across Min colonies, defined by differential adenoma multiplicity, the Min mouse is especially useful for elucidating underlying modifiers of disease susceptibility (Kwong and Dove 2009). In various studies, investigators have observed modifications of adenoma multiplicity stemming from both spontaneously occurring variants within the C57BL/6J (B6/J) strain, and through crosses with different mouse strains. Through these approaches, a number of loci that can substantially affect adenoma multiplicity were identified, and aptly termed

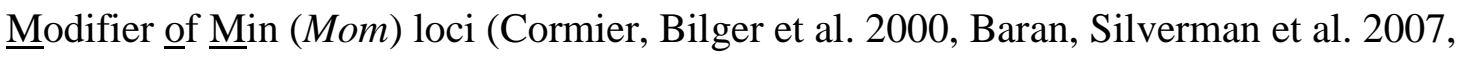
Kwong and Dove 2009). Despite the contributions of these studies, the extensive genetic variance between different inbred strains results in a large amount of "background noise," making it more difficult to resolve genetic modifiers of consequence. Thus, it may be advantageous to focus efforts to uncover modifiers of Min on strains more closely related to $\mathrm{B} 6 / \mathrm{J}$. To that end, the $\mathrm{C} 57 \mathrm{BL} / 6 \mathrm{~N}(\mathrm{~B} 6 / \mathrm{N})$ background is of interest as a related but divergent substrain of C57BL/6 that has now been separated from the B6/J substrain for hundreds of generations (Bryant 2011). During the course B6/J and B6/N divergence, the two substrains have accumulated distinct genotypes and phenotypes spanning various biological systems, ranging from neurobehavioral to immune responses (Bryant 2011, 
Fontaine and Davis 2016). Unfortunately, these substrains are often used interchangeably, and therefore important distinctions that may strongly influence a phenotype of interest are ignored. However, a keen awareness of these distinctions could provide leverage to uncover novel modifiers of complex traits. To date, it is unclear whether the B6/J and B6/N substrains have distinct modulatory effects on the Min phenotype as a result of their underlying divergence.

More recently, the gut microbiota (GM) has also emerged as an important factor in complex disease traits such as Inflammatory Bowel Disease (IBD) and CRC (Rubin, Shaker et al. 2012, Louis, Hold et al. 2014). The GM was initially identified as an important factor through associative studies, where investigators linked depletion or enrichment of certain taxa to disease status (Scanlan, Shanahan et al. 2008, Ohigashi, Sudo et al. 2013). Studies in animal models with quantitative complex disease traits have shed further light on the dynamic relationship between host and microbe. In a recent study, $i l 10^{-/-}$mice, which develop intestinal inflammation due to loss of immunoregulatory IL-10, were colonized with three distinct GM communities. The isogenic mice subsequently developed differing severity of disease based on GM colonization, corroborating the important role for intestinal microbial communities (Hart, Ericsson et al. 2017). Moreover, germ-free Min mice have a significantly lower adenoma burden than their colonized counterparts, suggesting that the GM influences the Min phenotype (Li, Kundu et al. 2012). Despite indications that the GM affects the Min phenotype, further studies are required to determine how different complex communities contribute to variability across Min colonies. 
In this study, we addressed two questions in an effort to further our understanding of different factors contributing to phenotypic variability across Min colonies. First, we aimed to determine how the $\mathrm{B} 6 / \mathrm{N}$ genetic background modifies adenoma multiplicity in comparison to the B6/J strain. Furthermore, we asked whether colonization with distinct complex GM communities further modulates adenoma multiplicity. We established F1 Min cohorts using Min males from the original colony at the McArdle Laboratory of the University of Wisconsin (C57BL/6JMlcr-Apc ${ }^{M i n} / \mathrm{Mmmh}$ abbrv. B6/JD), and either B6/J or B6/N wild-type females. F1 offspring from the B6/N lineage (B6NB6JDF1) were rederived using surrogates harboring two different complex GMs, such that isogenic mice were born with two distinct GMs. We describe differential adenoma suppression mediated by the B6/J and B6/N strains across GM-controlled groups, and further demonstrate GM-mediated phenotype modulation in isogenic mice. Through these approaches, we develop a novel platform for identifying genetic and bacterial modifiers of the Min phenotype, and concurrently illustrate how complex variables shape complex quantitative traits in a classic cancer model.

\subsection{Methods}

\subsubsection{Ethics Statement}

Animal studies were conducted in an Association for Assessment and Accreditation of Laboratory Animal Care International (AAALAC) accredited facility based on the guidelines provided by the Guide for the Care and Use of Laboratory Animals. All animal studies were approved by the University of Missouri Institutional Animal Care and Use Committee. 


\subsubsection{Animals}

Frozen C57BL/6JMlcr-Apc ${ }^{M i n} / \mathrm{Mmmh}(\mathrm{B} 6 / \mathrm{JD})$ embryos from the original Min colony at McArdle Laboratory, University of Wisconsin were acquired and rederived in our facility at the University of Missouri using CD-1 surrogate dams with a GM representing The Jackson Laboratory $\left(\mathrm{Crl}: \mathrm{CD} 1_{\mathrm{GM} 1}\right)$, previously generated in our laboratory (Hart, Ericsson et al. 2018). C57BL/6J-Apc ${ }^{\operatorname{Min}}(\mathrm{B} 6 / \mathrm{J})$ were acquired from the Jackson Laboratory. Both B6/JD and B6/J colonies were maintained at the University of Missouri as separate colonies. To generate B6JB6JDF1-Apc ${ }^{M i n}$ mice, 6-8 week old B6/J$A p c^{+/+}$females were mated with 6-8 week old B6/JD-Apc ${ }^{+/ M i n}$ males. Four to five week old female C57BL/6NHsd (B6/N) were purchased from Envigo (Indianapolis, IN). To generate B6NB6JDF1-Apc ${ }^{+/ \text {Min }}$ mice, in vitro fertilization (IVF) was performed as described previously using five to eight week old B6/N females and B6/JD males (Takeo T 2011). Presumed zygotes were then placed in a KSOM dish followed by incubation for 24 hours to allow advancement to the two-cell stage (Biggers JD 2000). In order to establish B6NB6JDF1-Apc ${ }^{+/ M i n}$ with two distinct complex GMs, two cohorts of CD-1 surrogate dams were used as embryo transfer recipients to perform Complex Microbiota Targeted Rederivation (CMTR) (Hart, Ericsson et al. 2018). CD-1 females harboring a GM (Hsd:CD $\left.1_{\mathrm{GM} 4}\right)$ were previously acclimated and maintained as a colony at the University of Missouri prior to their use as surrogates. The aforementioned $\mathrm{Crl}: \mathrm{CD} 1_{\mathrm{GM} 1}$ females harboring a GM representing The Jackson Laboratory were used as the second cohort, such that half of the B6NB6JDF1-Apc ${ }^{M i n}$ embryos were transferred into CD-1 $1_{\mathrm{GM} 1}$ surrogates, and half into $\mathrm{CD}-1_{\mathrm{GM} 4}$ surrogates. All female $\mathrm{CD}-1$ embryo recipients were mated with vasectomized $\mathrm{CD}-1_{\mathrm{GM} 1}$ or $\mathrm{CD}-1_{\mathrm{GM} 4}$ males, respectively, and those that were 
copulatory plug-positive were used for embryo transfer. CD-1 females were anesthetized via IM injection of ketamine/xylazine cocktail at $5.5 \mathrm{mg}$ and $1 \mathrm{mg}$ per $100 \mathrm{~g}$ body weight respectively. A dorsal midline incision was made and the uterine oviducts located by dissecting through the retroperitoneal muscle. Embryos in 3 to $5 \mu 1$ of media were injected into the oviducts using a glass hand-pipette. Skin incisions were closed with sterile surgical staples and mice received a subcutaneous injection of $2.5 \mathrm{mg} / \mathrm{kg}$ of body weight flunixin meglumine (Banamine $\left.{ }^{\circledR}\right)$ prior to recovery on a warming pad.

Surrogate dams were allowed to naturally deliver pups such that resulting B6NB6JDF1-Apc ${ }^{\text {Min }}$ would acquire the GM of their respective surrogate dams. Thus,

these mice are denoted as B6NB6JDF1-Apc ${ }^{\text {Min }}{ }_{\mathrm{GM} 1}$ and B6NB6JDF1-Apc ${ }^{\mathrm{Min}}{ }_{\mathrm{GM}}$. All mice were group-housed according sex, genetic lineage, and acquired GM (CMTR pups) in micro-isolator cages on ventilated racks (Thoren, Hazelton, PA). All mice had ad libitum access to 5058 irradiated breeder chow (LabDiet, St. Louis, MO) and acidified autoclaved water, maintained on paper chip bedding (Shepherd Specialty Papers, Watertown, TN) with 14:10 light:dark cycle. For genotyping, B6/J, B6/JD, and all F1 mice were ear-punched at 21 days of age (weaning). DNA was extracted using the “HotSHOT" genomic DNA preparation method as described (Truett, Heeger et al. 2000).

\subsubsection{Adenoma Counts}

At 100 days of age, mice requiring adenoma counts were euthanized via $\mathrm{CO}_{2}$ asphyxiation. Following exposure through the abdominal cavity, whole small and large intestines were incised longitudinally, flushed with saline and placed on bibulous paper with the luminal side facing up for formalin fixation. Grossly visible adenomas were counted manually using a Leica M165FC microscope at 1.25x magnification. 


\subsubsection{Sample collection and DNA extraction for 16S rRNA sequencing}

Fecal samples were collected from B6/J, B6/JD, B6JB6JDF1, and both CMTRderived B6NB6JDF1 groups at 1 month of age. Mice were placed in an empty autoclaved cage until they defecated naturally. Two fecal pellets per mouse were collected aseptically and placed in a $2 \mathrm{~mL}$ round-bottom tube containing $800 \mu \mathrm{l}$ of lysis buffer as described (Ericsson, Davis et al. 2015) and a $0.5 \mathrm{~cm}$ diameter stainless steel bead (Grainger, Lake Forest, Il). Fecal samples were mechanically disrupted using a TissueLyser II (Qiagen, Venlo, Netherlands) for 3 minutes at $45 \mathrm{~Hz}$, followed by incubation at $70^{\circ} \mathrm{C}$ for 20 minutes with periodic vortexing. DNA extraction from fecal pellets, cecal contents, and ileal epithelium for 16S rRNA sequencing was performed using a DNeasy Blood \& Tissue Kit ${ }^{\circledR}$ (Qiagen) as previously described (Ericsson, Davis et al. 2015).

\subsubsection{S library preparation and sequencing}

DNA extracted from fecal pellets was sent to the University of Missouri DNA Core facility (Columbia, MO) for further processing. An amplicon library of the hypervariable V4 region of the bacterial 16S rRNA gene was generated using the U515F/806R primer set as described previously (Caporaso, Lauber et al. 2010). Amplicons were sequenced on the Illumina MiSeq platform under the described conditions (Montonye, Ericsson et al. 2018).

\subsubsection{Informatics Processing}

Trimming, assembly, binning, and annotation of DNA sequences was performed at the University of Missouri Informatics Research Core Facility (Columbia, MO). 
Quality control of DNA, assembly of contiguous sequences, sequence removal after trimming for base quality, and chimera removal were completed as described (Montonye, Ericsson et al. 2018). All remaining sequences were assigned to operational taxonomic units (OTUs) by de novo OTU clustering based on $97 \%$ nucleotide similarity. OTUs were annotated using BLAST (Altschul, Madden et al. 1997) against the SILVA database (Ritari, Salojarvi et al. 2015) of 16s rRNA sequences and taxonomy. OTU relative abundances were subject to a $1 / 4$ root transformation prior to visualization using Principal Component Analysis (PCA). PCA visualization and $\alpha$-diversity indices (Chao1 and Shannon) were acquired using Past 3.12 software (Hammer, Harper et al. 2001). Open access Metaboanalyst 3.0 was used to generate heat maps of microbiome data based on Euclidian distance measurements and the Ward clustering algorithm applied to the cube root-transformed dataset.

\subsubsection{Statistical analysis}

Statistical analyses of all adenoma counts were performed using Analysis of Variance (ANOVA) with the Student Newman-Keul post-hoc test in Sigma Plot 14.0 (Systat Software Inc., Carlsbad CA). For statistical comparisons of bacterial phyla and $\alpha$ diversity indices, the student's t-test was used. Metaboanalyst 3.0 was used to determine statistically significant OTUs via the Student's t-test. For adenoma counts, phyla, and $\alpha$ diversity, $p$-values $<0.05$ were considered statistically significant, while FDR-values $<$ 0.05 were considered significantly different for bacterial OTUs. GraphPad Prism 8 was used to generate all scatter plots, bar graphs, and box plots.

\subsection{Results}




\subsubsection{B6JB6JDF1-Apc ${ }^{M i n}$ mice exhibit partial repression of the B6/JD adenoma phenotype}

The B6/JD and B6/J lineages were maintained as distinct colonies within the same facility at the University of Missouri. At 100 days of age, Min mice from both parental lines were humanely sacrificed for adenoma counts. We observed significantly fewer adenomas in the $\mathrm{B} 6 / \mathrm{J}$ line (44.2 \pm 16.5 adenomas) compared to the $\mathrm{B} 6 / \mathrm{JD}$ line $(106.6 \pm 24.0$ adenomas; $p<0.001$ ) (Fig. 1). To determine the capacity for the B6/J genetic background to repress the $\mathrm{B} 6 / \mathrm{JD}$ phenotype, wild-type female $\mathrm{B} 6 / \mathrm{J}$ mice were mated with Min male B6/JD mice to generate B6JB6JDF1-Apc ${ }^{\text {Min }}$ offspring. F1 offspring were similarly sacrificed at 100 days of age for manual adenoma counts. We found that the F1 generation displayed an intermediate adenoma phenotype (73.9 \pm 21.1 adenomas), as they developed significantly fewer adenomas that the B6/JD line and significantly more adenomas than the B6/J parents $(p<0.001)$ (Fig. 1).

\subsubsection{B6/ $\mathrm{N}$ repression of adenomas is modulated by the gut microbiota}

Given the divergence between the B6/J and B6/N lineages, we first asked whether the $\mathrm{B} 6 / \mathrm{N}$ genetic background would exert similar adenoma repression to the $\mathrm{B} 6 / \mathrm{J}$ genetic background when crossed with the B6/JD lineage. Furthermore, we aimed to determine whether distinct complex GM communities would modulate the effects of the B6/N genetic background in the context of adenoma multiplicity. We performed Complex Microbiota Targeted Rederivation (CMTR) to establish B6NB6JDF1-Apc ${ }^{\text {Min }}$ mice with two distinct complex GMs; a low-richness community (GM1) and a high-richness community (GM4) using CD-1 surrogate dams with GMs that originally represented The Jackson Laboratory (GM1) or Envigo (GM4) (Fig. 2A). At 100 days of age, both 
B6NB6JDF1- $A p c^{M i n}{ }_{\mathrm{GM} 1}$ and B6NB6JDF1-Apc ${ }^{\mathrm{Min}}{ }_{\mathrm{GM}}$ groups were sacrificed for adenoma counts, and compared to B6JB6JDF1-Apc ${ }^{M i n}$. We found that Min B6NB6JDF1 mice, regardless of GM colonization, developed significantly fewer SI adenomas than Min B6JB6JDF1 mice (Fig. 2B). We also observed a significant effect of the GM, where Min B6NB6JDF1 mice colonized with GM4 (53.1 \pm 11.2 adenomas) developed more adenomas than those colonized with GM1 (34.3 \pm 6.8 adenomas). Thus, the B6/N genetic background demonstrated stronger suppression of the B6/JD phenotype compared to the B6/J background. Moreover, the degree of B6/N-mediated suppression was modulated by GM colonization.

\subsubsection{Distinct GMs confer differential adenoma susceptibility}

Given the significant effect of GM colonization on adenoma numbers in B6NB6JDF1-Apc Min mice, we aimed to characterize GM1 and GM4. Fecal samples were collected from $\mathrm{B} 6 \mathrm{NB} 6 \mathrm{JDF} 1_{\mathrm{GM} 1}$ and $\mathrm{B} 6 \mathrm{NB} 6 \mathrm{JDF} 1_{\mathrm{GM} 4}$ mice at 1 month of age and subject to sequencing of the $16 \mathrm{~S}$ rRNA microbial gene to determine relative abundances of all detected bacterial phyla and operational taxonomic units (OTUs). To visualize overall GM community similarity of parental lineages and F1 offspring, a Principal Component Analysis (PCA) was used (Fig. 3A). Notably, B6JB6JDF1 and B6NB6JDF1 $1_{\mathrm{GM} 1}$ demonstrate similar overall GM communities, both of which are distinct from B6NB6JDF1- $A p c^{M i n}{ }_{\text {GM4 }}$ mice. B6JB6JDF1 mice also have a distinct GM compared to the B6/J and B6/JD parental lineages from which they were generated. Mice colonized with GM4 had increased Chao1 and Shannon indices, indicating increased richness and diversity compared to GM1, respectively (Fig. 3B). We found that GM4 was enriched with the phyla Actinobacteria, Cyanobacteria, Deferribacteres, Proteobacteria, and had 
an increased Firmicutes to Bacteroidetes ratio relative to GM1. Meanwhile, GM1 was enriched for Patescibacteria, Tenericutes, and Verrucomicrobia (Fig. 3C). Overall, 60 OTUs were determined to be differentially abundant between GM1 and GM4 based on a False Discovery Rate (FDR) $<0.05$ (Table S1). A heat map was used to show foldchange of the 25 most significantly modulated OTUs, and further describes the distinct nature of GM1 and GM4 through hierarchical clustering analysis (Fig. 3D). To summarize, GM1 and GM4 are distinct complex GM communities at the phylum and OTU level, and confer differential susceptibility to adenoma development in B6NB6JDF1-Apc ${ }^{\text {Min }}$ mice.

\subsection{Discussion}

For almost three decades, the Min mouse has served as one of the most widely used animal disease models, providing a wealth of information about underlying mechanisms and potential therapeutic targets in CRC. The quantitative nature of the Min phenotype has been particularly useful for identifying potential modifiers of adenoma initiation. In this study, we aimed to determine whether two related inbred genetic strains, C57BL/6J and C57BL/6N, differentially modulate the Min adenoma phenotype, and to establish whether distinct GM communities could modify the Min phenotype. In doing so, we provide a platform for the discovery of novel genetic and microbial modifiers of Min, and demonstrate the multifaceted determinants of adenoma initiation comprising the commonly observed phenotypic variability associated with the Min mouse.

We crossed C57BL/6JD-Apc ${ }^{\text {Min }}$ (B6/JD) males from the original Min colony of

the McArdle Laboratory at the University of Wisconsin with C57BL/6J (B6/J) females to 
generate a B6JB6JDF1 Min cohort (Fig. 1). We also used C57BL/6N (B6/N) females to generate a B6NB6JDF1 Min cohort, such that we could compare the two F1 groups to determine their modulatory effects on adenoma initiation. Furthermore, B6NB6JDF1 embryos were rederived using surrogate dams harboring two different GMs (Fig. 2A): GM1 resembles the GM of B6JB6JDF1 mice, while GM4 represents a highly distinct community (Fig. 3A), thereby enabling interrogation of the GM as an environmental modifier of Min (Hart, Ericsson et al. 2018). We found that the B6/J background conferred moderate suppression of the original B6/JD adenoma phenotype, resulting in an intermediate F1 phenotype relative to parental strains (Fig. 1) (colonic adenoma counts, Fig. S1). We also observed that both GM1- and GM4-colonized B6NB6JDF1 mice developed fewer adenomas than B6JB6JDF1 animals (Fig. 2B), indicating that the B6/N background has a significantly stronger capacity to suppress adenomas than the B6/J background. Despite the relative relatedness of the B6/J and B6/N strains, these results suggest that during the course of their divergence, each strain has acquired variants that confer differential susceptibility to adenoma initiation. A number of studies have described phenotypic differences between $\mathrm{B} 6 / \mathrm{J}$ and $\mathrm{B} 6 / \mathrm{N}$ related to behavior, metabolism, and immunity. These changes are largely attributed to the approximated 150 homozygous SNPs detected between the substrains, including the classically referenced mutation in the nicotinamide nucleotide transhydrogenase (Nnt) gene in B6/J (Bryant 2011, Fontaine and Davis 2016). Given that these substrains have accumulated variants that differentially modify the Min phenotype, documented genomic differences between $\mathrm{B} 6 / \mathrm{J}$ and $\mathrm{B} 6 / \mathrm{N}$ can be used as a platform to identify additional modifiers of Min. 
Moreover, these data provide further evidence that investigators should be highly cognizant of specific substrains used for complex trait analysis.

While both GM1- and GM4-colonized B6NB6JDF1 mice had fewer adenomas than B6JB6JDF1 mice, we noted a significant modulatory effect of the GM, where GM4colonized mice developed more adenomas than their GM1 counterparts (Fig. 2B) (Colonic adenoma counts, Fig. S2). Thus, Min mice with genetically identical backgrounds may have phenotypic variability based on GM colonization. Nextgeneration sequencing (NGS) of the microbial 16S rRNA gene enables identification and relative quantification of all detected Operational Taxonomic Units (OTUs) (Ward, Weller et al. 1990). Given the apparent phenotypic effects of the GM, we utilized this approach to characterize the GMs of all F1 mice, as well as the B6/J and B6/JD parental lines. We subsequently performed Principal Component Analysis (PCA) to visualize $\beta$ diversity and thus infer similarity of overall GM community structure between different groups (Fig. 3A). As mentioned previously, PCA demonstrates the relative similarity of the GMs of B6JB6JDF1 and B6NB6JDF1 $1_{\mathrm{GM} 1}$ mice. Thus, we adequately controlled for GM variability when comparing the two groups, confirming that the $\mathrm{B} 6 / \mathrm{N}$ genetic background mediates enhanced adenoma suppression compared to B6/J. PCA also demonstrates the distinct nature of GM1 and GM4 that resulted in differential adenoma susceptibility, as anticipated based on the surrogate dams used for rederivation. Further analysis of GM1 and GM4 revealed differentially abundant phyla, which included a significant increase in the Firmicutes:Bacteroidetes ratio in GM4 relative to the GM1 (Fig. 3B). Interestingly, previous reports analyzing samples from human IBD and CRC patients have found similar trends when compared to healthy controls (Bamola, Ghosh et 
al. 2017). We also noted enrichment of several members of the Proteobacteria phylum, including sulfidogenic Desulfovibrio and Bilophila sp (Fig. 3D). Though various studies have suggested a potentially carcinogenic role for these bacteria (Attene-Ramos, Wagner et al. 2006, Hellmich and Szabo 2015, Guo, Yu et al. 2016), studies that directly interrogate their phenotypic influence in the context of a complex GM are required. Of note, we also found that the GM of B6JB6JDF1 mice primarily clustered separately from their B6/J and B6/JD parents. This phenomenon demonstrates the possibility of GM drift across multi-generational experiments using genetic crosses. Investigators should therefore remain mindful of these potential changes, particularly when analyzing quantitative phenotypes that may be subject to the GM.

The Min mouse is a classic quantitative trait with various biological systems contributing to composite adenoma multiplicity. This model has particularly served those in the genetics community seeking to identify genetic modifiers of adenoma susceptibility. In this study, we demonstrate differential adenoma suppression mediated by the $\mathrm{B} 6 / \mathrm{J}$ and $\mathrm{B} 6 / \mathrm{N}$ genetic backgrounds, establishing the need for investigators to be highly attentive to specific substrains when interpreting quantitative trait analyses. Furthermore, we show that the GM may also modify complex traits such as Min adenoma multiplicity. Thus, we not only provide a platform for the discovery of novel genetic and microbial modifiers of Min, but also establish a complex combination of physiological determinants comprising quantitative trait outcomes.

\subsection{Author Contributions and Acknowledgements}


JM and JAL designed the described experiments. JM and FA performed all experiments. The authors wish to acknowledge Nathan Bivens and the MU DNA Core for their assistance with 16S rDNA sequencing, and Bill Spollen, Christopher Bottoms and the MU Informatics Research Core Facility for assistance with 16S rRNA sequencing analysis. The authors would also like to acknowledge the MU Office of Animal Resources for their role in animal husbandry and maintenance.

\subsection{Financial Support}

This research was funded by grants from the National Institutes of Health to the Mutant Mouse Resource and Research Center at the University of Missouri (U42 OD010918), and by the University of Missouri to Dr. James Amos-Landgraf (Startupfunding). JM was supported by NIH T32 OD011126. 


\subsection{Figures}

Figure 1. B6JB6JDF1-Apc ${ }^{M i n}$ mice display an intermediate adenoma phenotype.

Scatter plots comparing mean $( \pm \mathrm{SD})$ small intestinal $(\mathrm{SI})$ adenoma counts of the original B6- $A p c^{M i n}$ colony generated at UW McArdle Laboratory (B6/JD), B6- $A p c^{M i n}$ mice acquired from The Jackson Laboratory and maintained at University of Missouri (B6/J), and their F1 offspring (B6JB6JDF1) (B6/JD, $\mathrm{n}=19 ; \mathrm{B} 6 / \mathrm{J}, \mathrm{n}=22 ; \mathrm{B} 6 \mathrm{JB} 6 \mathrm{JDF} 1, \mathrm{n}=19)$. $* * * p<0.001$; ANOVA with the Student Newman-Keuls method.

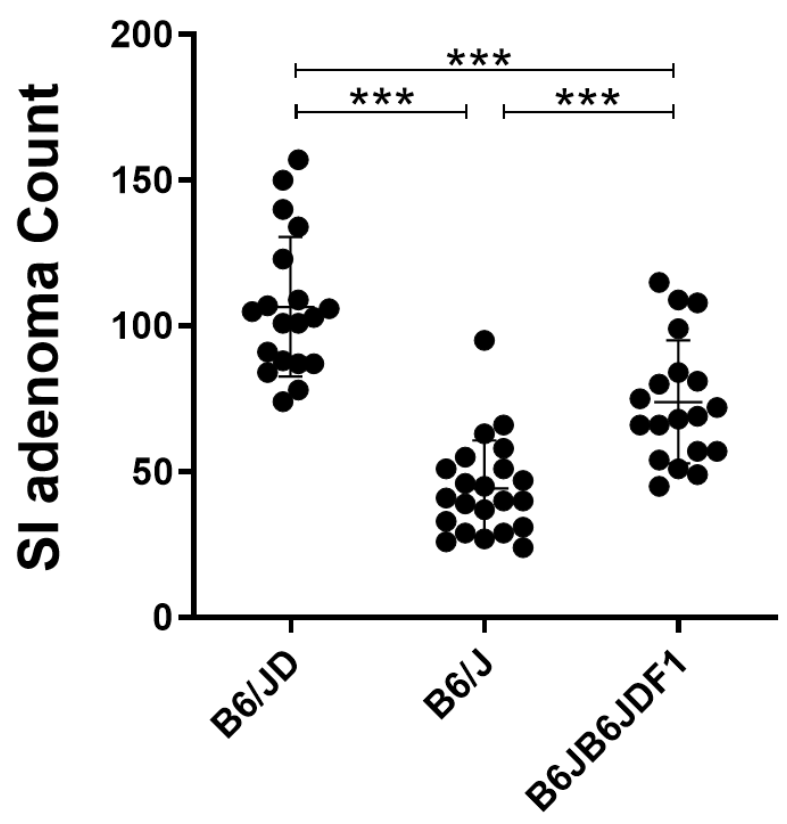


Figure 2. The C57BL/6NHsd genetic background and the gut microbiota modulate adenoma repression in $A p c^{M i n}$ mice. A, F1 embryos from C57BL/6NHsd-Apc ${ }^{+/+}$and C57BL/6JD-Apc $c^{+/ M i n}$ parental lines were generated via IVF, and transplanted into CD-1 surrogate dams harboring two distinct complex GM profiles; GM1 and GM4. Offspring maintain their F1 hybrid genetic lineage while inheriting a GM from respective surrogate CD-1 dams. (B6NB6JDF1-Apc ${ }^{M i n}{ }_{\mathrm{GM} 1}, \mathrm{n}=9$; B6NB6JDF1-Apc $\left.{ }^{M i n}{ }_{\mathrm{GM} 4}, \mathrm{n}=8\right)$. B, Scatter plots comparing mean ( \pm SD) SI adenoma counts of B6JB6JDF1-Apc ${ }^{\text {Min }}$, B6NB6JDF1$A p c^{M i n}{ }_{\mathrm{GM} 1}$, and B6NB6JDF1-Apc ${ }^{M i n}{ }_{\mathrm{GM} 4}$ mice. ${ }^{*} p<0.05, * * P<0.01, * * * p<0.001$; ANOVA with the Student Newman-Keuls method.

A

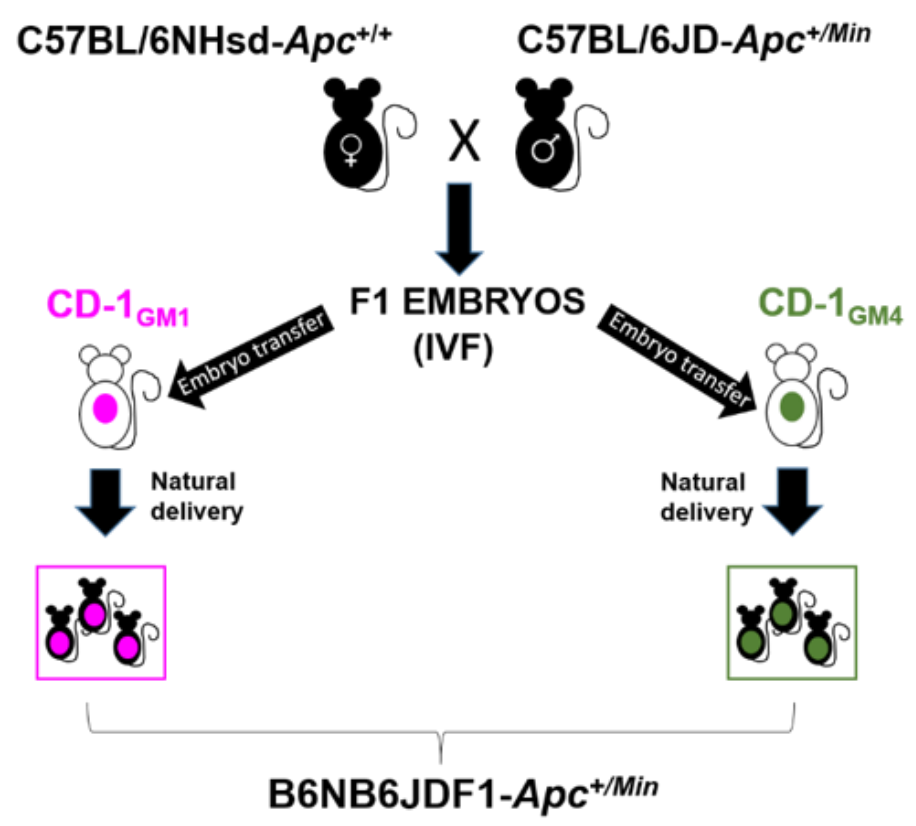


B

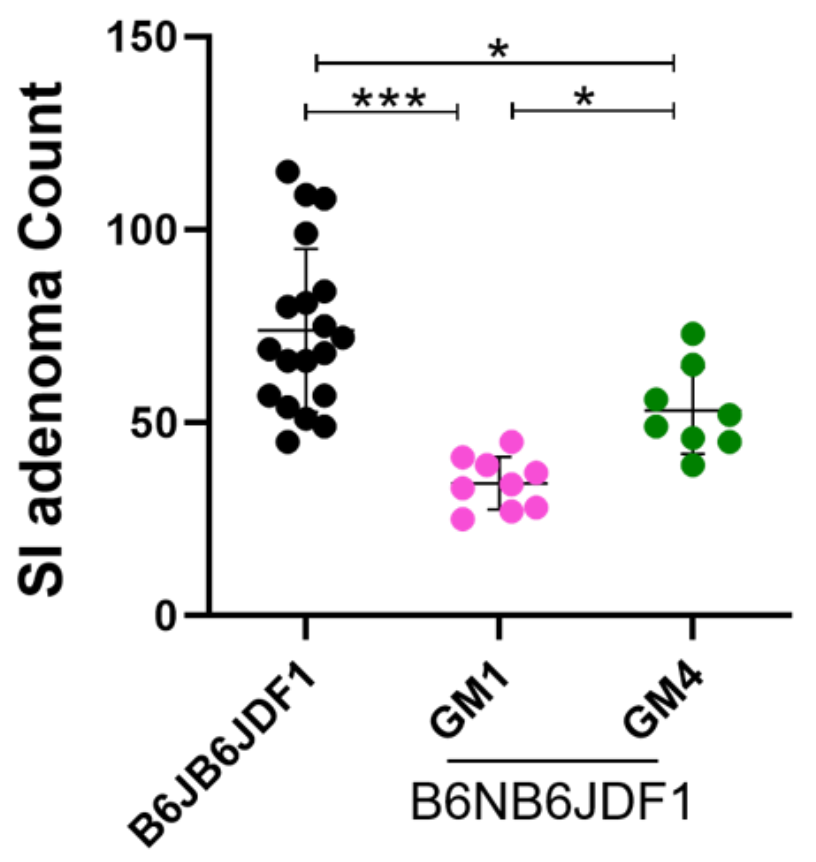


Figure 3. Distinct complex gut microbiota communities contribute to differential

adenoma counts. A, Principal Component Analysis (PCA) representing differences in $\beta$ diversity at the Operational Taxanomic Unit (OTU) level between complex GM profiles of B6/J $(n=8)$ and B6/JD $(n=11)$ parental lines, B6JB6JDF1 offspring $(n=10)$, B6NB6JDF1-Apc ${ }^{M i n}{ }_{\mathrm{GM} 1}(\mathrm{n}=13)$, and B6NB6JDF1-Ap$c^{M i n}{ }_{\mathrm{GM} 4}$ mice $(\mathrm{n}=14) . \quad$ B, Differences in GM richness (Chao1 index) and $\alpha$-diversity (Shannon Index) are shown with Tukey's boxplots (GM1, $\mathrm{n}=9$, GM4, $\mathrm{n}=8)$. C, Bar charts representing relative abundances (mean \pm SEM) of phyla with detected significant differences between GM1 and GM4, in fecal samples from mice at 1 month of age (GM1, n =9;GM4, n= 8). D, Heatmap showing 25 taxa with significantly different $($ FDR $<0.05)$ fecal relative abundances between GM1 and GM4 at 1 month. Color intensity represents fold-change of each OTU. Hierarchical clustering based on Euclidean distances (top) demonstrates clustering of samples based on GM. All statistically significant OTUs and associated fold changes are represented in supplementary table $1 .{ }^{*} p<0.05, * * p<0.01, * * * p<0.001$;

Student's t-test.

A

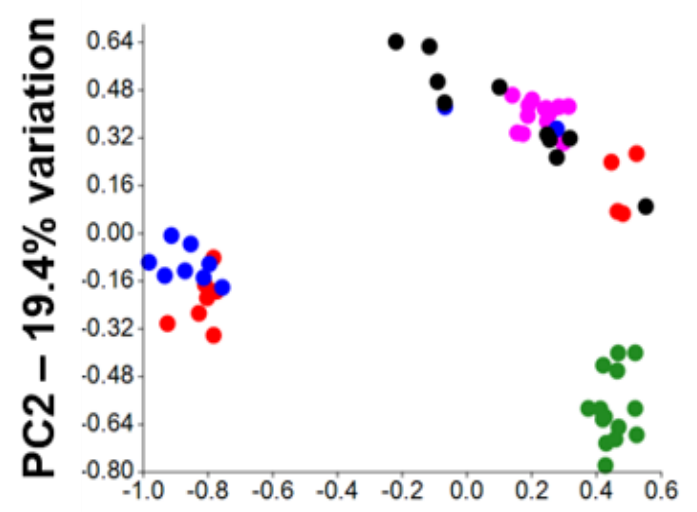

B6-Apc ${ }^{+/ M i n}$ - B6/J (parents) - B6/JD (parents) B6JB6JDF1

B6NB6JDF1 - GM4

PC1 - 31.3\% variation 
B
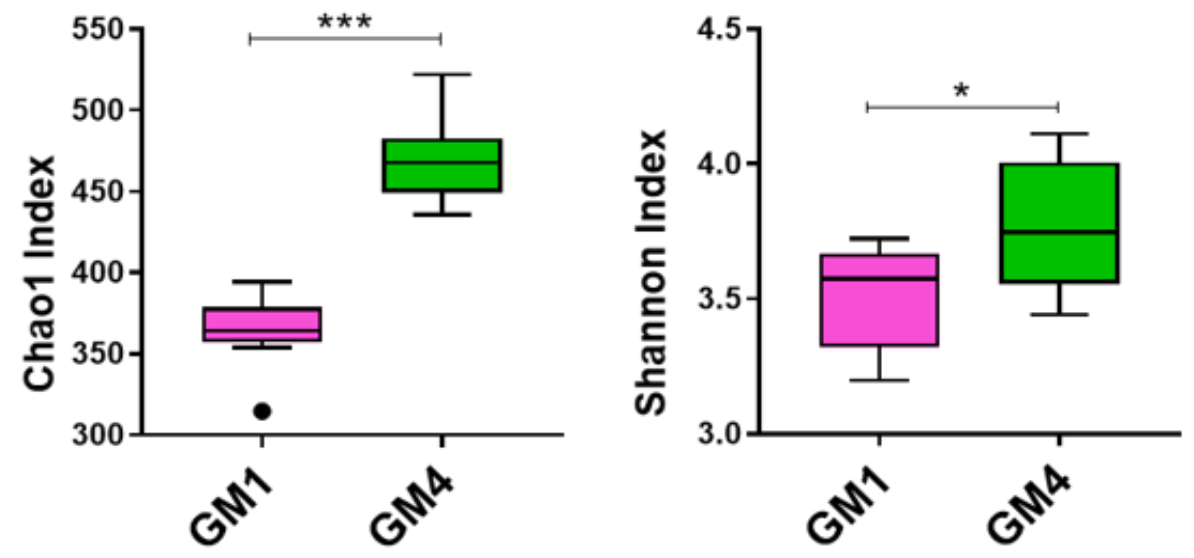
C
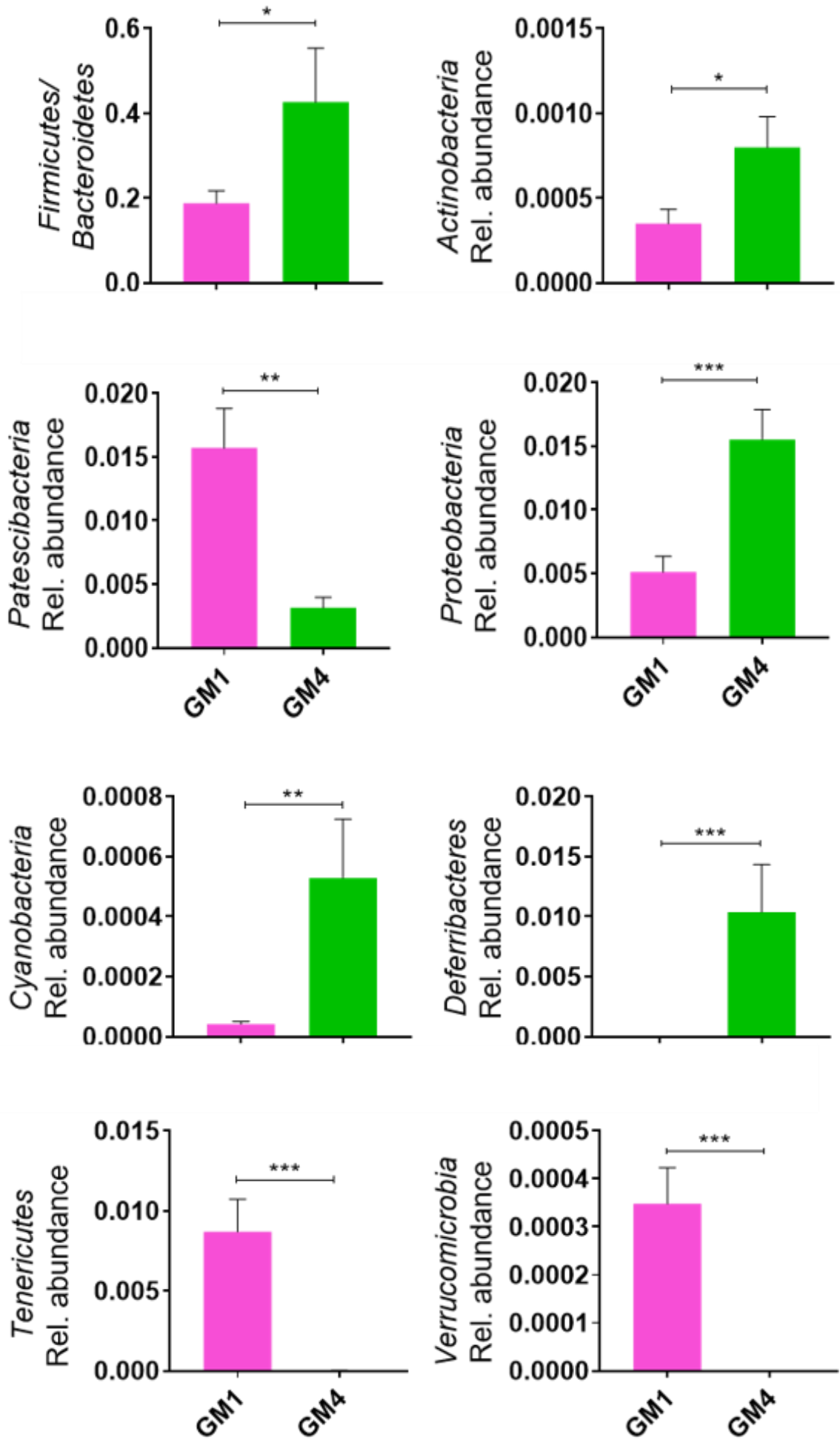
D

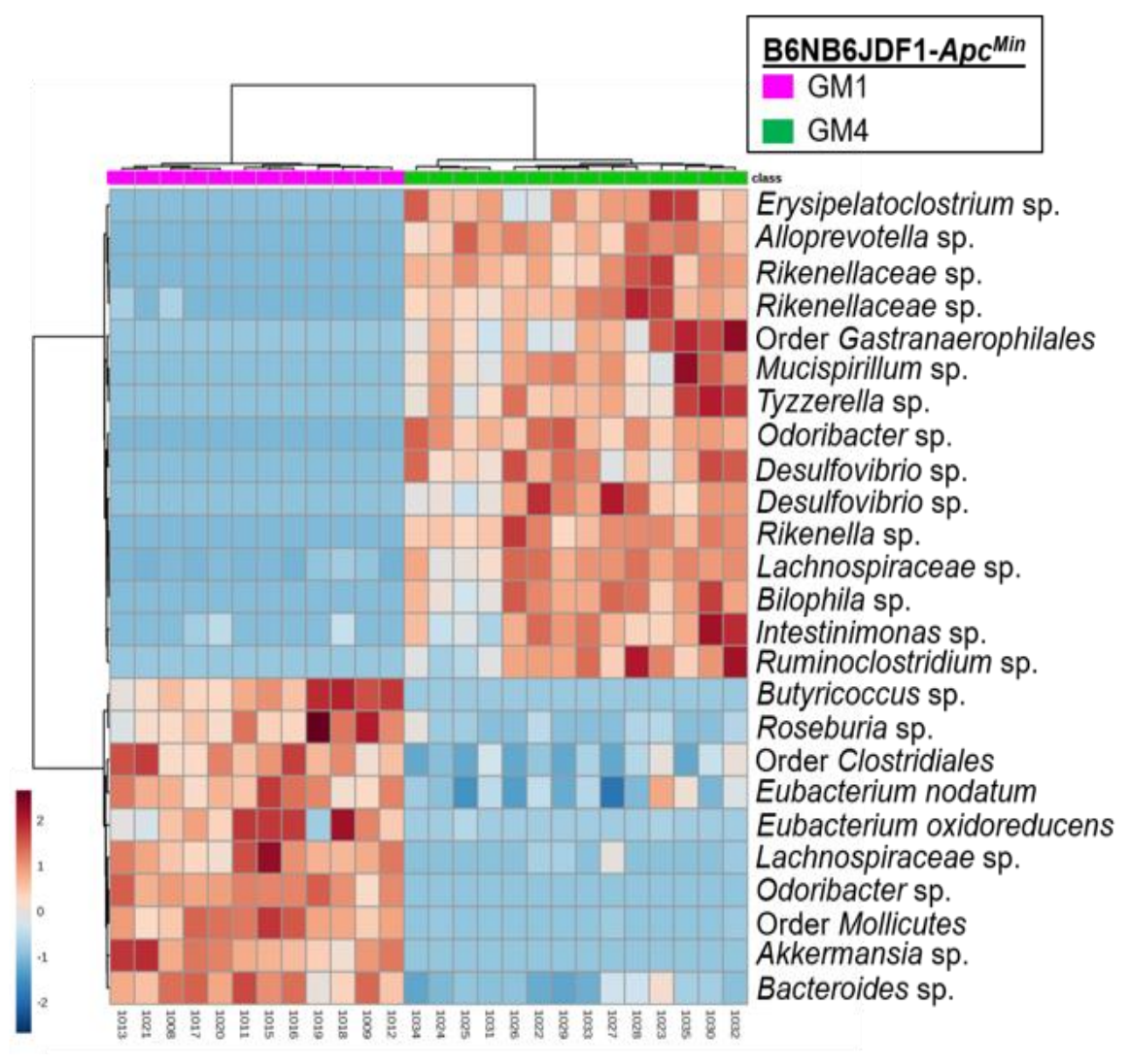


Supplementary Figure 1. Colonic adenoma numbers in B6/J and B6/JD parental lines and B6JB6JDF1-Apc ${ }^{M i n}$ offspring. Scatter plots comparing mean $( \pm$ SD) colonic adenoma counts of the original B6-Apc ${ }^{\mathrm{Min}}$ colony generated at UW McArdle Laboratory (B6/JD), B6-Apc $c^{M i n}$ mice acquired from The Jackson Laboratory and maintained at University of Missouri (B6/J), and their F1 offspring (B6JB6JDF1) (B6/JD, n = 19; B6/J, $\mathrm{n}=22 ;$ B6JB6JDF1, $\mathrm{n}=19$ ). ANOVA with the Student Newman-Keuls method.

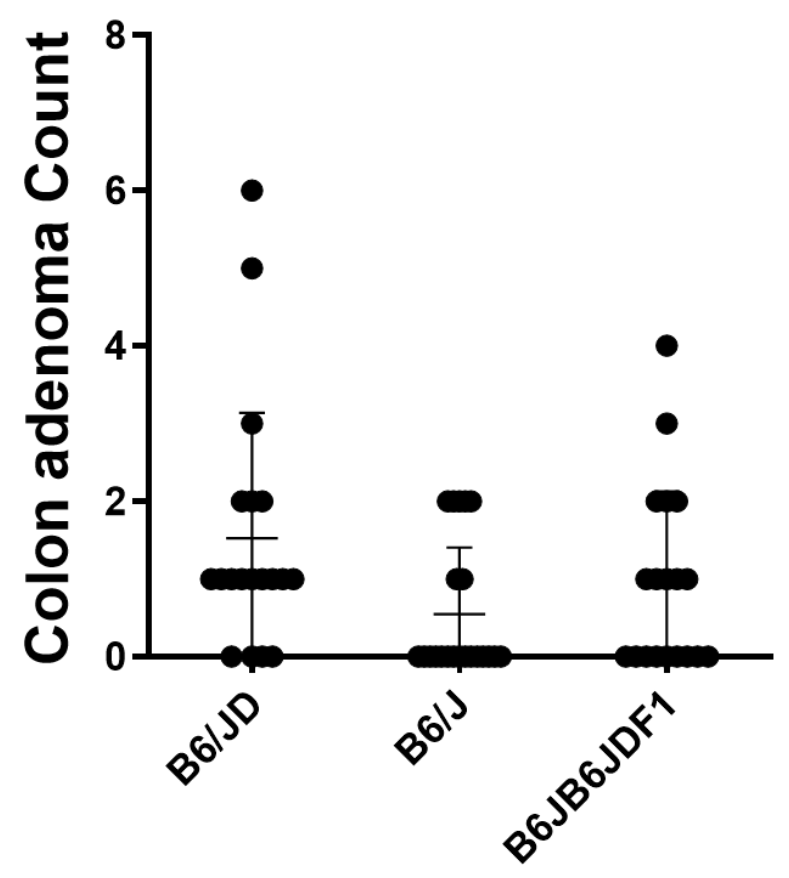


Supplementary Figure 2. Colonic adenoma counts in rederived F1 Apc ${ }^{M i n}$ mice. Scatter plots comparing mean $( \pm \mathrm{SD})$ colonic adenoma counts of B6JB6JDF1-Apc ${ }^{\text {Min }}$, B6NB6JDF1-Apc ${ }^{M i n}{ }_{\mathrm{GM} 1}$, and B6NB6JDF1-Apc ${ }^{\mathrm{Min}}{ }_{\mathrm{GM} 4}$ mice. ANOVA with the Student Newman-Keuls method.

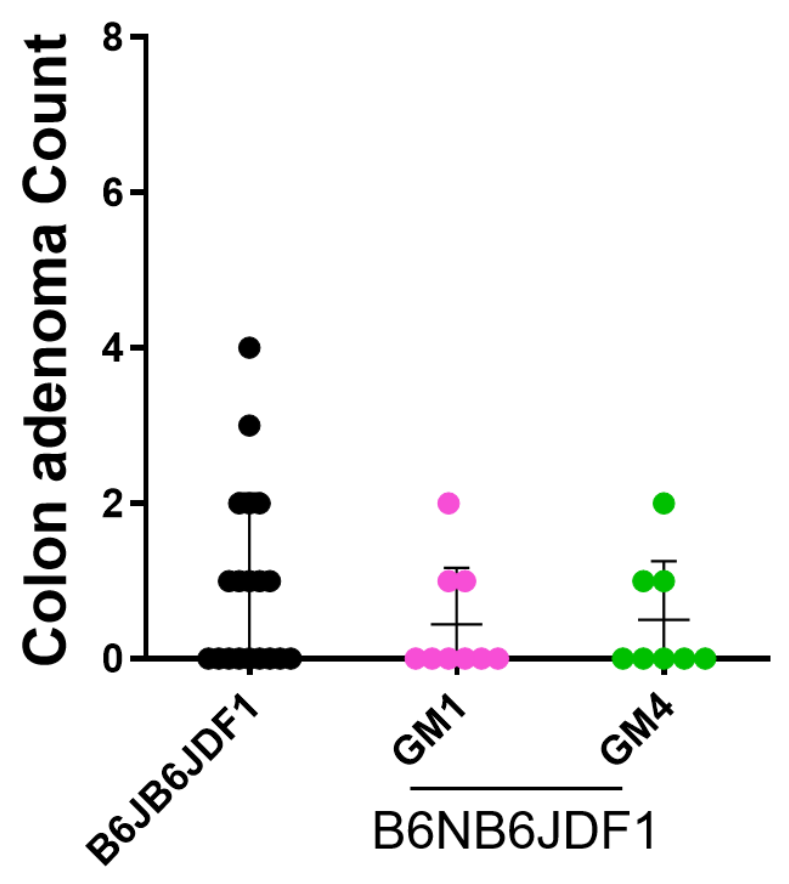




\subsection{Tables}

\section{Supplementary Table 1. Significantly different fecal OTUs between GM1 and GM4}

\begin{tabular}{|c|c|c|c|c|c|}
\hline \multicolumn{2}{|c|}{ Significant OTUs in B6NB6JDF1 mice $($ FDR $<0.05)$} & \multicolumn{2}{|c|}{$\begin{array}{c}\text { GM1 } \\
\text { relative to GM4 } \\
\end{array}$} & \multirow{2}{*}{$\begin{array}{c}\text { GM1 } \\
\begin{array}{c}\text { Mean Relative } \\
\text { Abundance } \\
( \pm \text { SEM })\end{array} \\
\end{array}$} & \multirow{2}{*}{$\begin{array}{c}\text { GM4 } \\
\text { Mean Relative } \\
\text { Abundance } \\
( \pm \text { SEM })\end{array}$} \\
\hline Phylum & OTU & FDR & $\begin{array}{c}\text { Mean } \\
\text { Fold } \\
\text { Change } \\
\end{array}$ & & \\
\hline Bacteroidetes & genus Odoribacter.1 & NA & NA & $0.00554 \pm 0.0006$ & ND \\
\hline Bacteroidetes & genus Odoribacter & NA & NA & ND & $0.0027 \pm 0.0004$ \\
\hline Bacteroidetes & genus Alloprevotella.1 & NA & NA & ND & $0.0326 \pm 0.0048$ \\
\hline Bacteroidetes & genus Rikenella & NA & NA & ND & $0.0026 \pm 0.0004$ \\
\hline Bacteroidetes & genus Rikenellaceae $\mathrm{RC} 9$ gut group & NA & NA & ND & $0.0020 \pm 0.0003$ \\
\hline Tenericutes & order Mollicutes RF39.1 & 3.69E-12 & 1342.3 & $0.0071 \pm 0.0012$ & $2.66 \mathrm{E}-06 \pm 1.47 \mathrm{E}-06$ \\
\hline Verrucomicrobia & genus Akkermansia & NA & NA & $\begin{array}{c}0.0003 \\
\pm 5.93 \mathrm{E}-05 \\
\end{array}$ & ND \\
\hline Firmicutes & $\begin{array}{l}\text { genus Lachnospiraceae NK4A136 } \\
\text { group.3 }\end{array}$ & $3.41 \mathrm{E}-11$ & 0.002448 & $0.0399 \pm 0.0074$ & $0.1325 \pm 0.0234$ \\
\hline Bacteroidetes & $\begin{array}{l}\text { genus Rikenellaceae } \mathrm{RC} 9 \text { gut } \\
\text { group.1 }\end{array}$ & $5.21 \mathrm{E}-10$ & 0.012343 & $3.77 \mathrm{E}-06 \pm 2.03 \mathrm{E}-06$ & $0.0005 \pm 9.30 \mathrm{E}-05$ \\
\hline Proteobacteria & genus Bilophila & NA & NA & ND & $0.0012 \pm 0.0002$ \\
\hline Firmicutes & genus Lachnospiraceae UCG-009 & 4.41E-09 & 35.276 & $0.0003 \pm 6.079 \mathrm{E}-05$ & $5.79 \mathrm{E}-06 \pm 3.95 \mathrm{E}-06$ \\
\hline Proteobacteria & genus Desulfovibrio.1 & NA & NA & ND & $0.0058 \pm 0.0012$ \\
\hline
\end{tabular}




\begin{tabular}{|c|c|c|c|c|c|}
\hline Firmicutes & genus Erysipelatoclostridium & NA & NA & ND & $7.82 \mathrm{E}-05 \pm 1.33 \mathrm{E}-05$ \\
\hline Bacteroidetes & genus Bacteroides.1 & $9.30 \mathrm{E}-09$ & 7.2568 & $0.1031 \pm 0.0102$ & $0.0138 \pm 0.0030$ \\
\hline Firmicutes & genus Butyricicoccus.1 & NA & NA & $0.0021 \pm 0.0005$ & ND \\
\hline Deferribacteres & genus Mucispirillum & NA & NA & ND & $0.0082 \pm 0.0024$ \\
\hline Firmicutes & genus Tyzzerella 4 & NA & NA & ND & $0.0003 \pm 7.12 \mathrm{E}-05$ \\
\hline Firmicutes & order Clostridiales. 1 & $2.23 \mathrm{E}-07$ & 10.095 & $0.0002 \pm 2.52 \mathrm{E}-05$ & $1.59 \mathrm{E}-05 \pm 5.16 \mathrm{E}-06$ \\
\hline Proteobacteria & genus Desulfovibrio & NA & NA & ND & $0.0010 \pm 0.0003$ \\
\hline Firmicutes & genus Roseburia.1 & 5.09E-06 & 60.418 & $0.0022 \pm 0.0008$ & $3.89 \mathrm{E}-05 \pm 2.14 \mathrm{E}-05$ \\
\hline Firmicutes & genus Intestinimonas & $6.99 \mathrm{E}-06$ & 0.043832 & $3.17 \mathrm{E}-06 \pm 1.51 \mathrm{E}-06$ & $0.0001 \pm 2.90 \mathrm{E}-05$ \\
\hline Firmicutes & genus Eubacterium nodatum & $7.53 \mathrm{E}-06$ & 3.7466 & $0.0005 \pm 4.57 \mathrm{E}-05$ & $0.0001 \pm 3.04 \mathrm{E}-05$ \\
\hline Firmicutes & genus Eubacterium oxidoreducens & $1.40 \mathrm{E}-05$ & 469.53 & $0.0018 \pm 0.0006$ & $1.78 \mathrm{E}-06 \pm 1.32 \mathrm{E}-06$ \\
\hline Cyanobacteria & order Gastranaerophilales.1 & NA & NA & ND & $0.0005 \pm 0.0002$ \\
\hline Firmicutes & genus Ruminiclostridium 6.1 & NA & NA & ND & $0.0015 \pm 0.0005$ \\
\hline Firmicutes & family Lachnospiraceae.5 & 2.69E-05 & 91.759 & $0.0032 \pm 0.0011$ & $2.92 \mathrm{E}-05 \pm 8.47 \mathrm{E}-06$ \\
\hline Firmicutes & $\begin{array}{l}\text { genus Lachnospiraceae } \mathrm{NC} 2004 \\
\text { group }\end{array}$ & NA & NA & ND & $0.0004 \pm 0.0001$ \\
\hline Bacteroidetes & family Muribaculaceae. 1 & 3.82E-05 & 5.0969 & $0.0001 \pm 1.52 \mathrm{E}-05$ & $1.73 \mathrm{E}-05 \pm 4.44 \mathrm{E}-06$ \\
\hline Proteobacteria & genus Parasutterella & NA & NA & ND & $5.76 \mathrm{E}-05 \pm 1.45 \mathrm{E}-05$ \\
\hline Bacteroidetes & family Muribaculaceae & 8.97E-05 & 0.45146 & $0.0021 \pm 0.0002$ & $0.0047 \pm 0.0005$ \\
\hline Firmicutes & order Clostridiales. 2 & NA & NA & ND & $8.11 \mathrm{E}-05 \pm 2.39 \mathrm{E}-05$ \\
\hline
\end{tabular}




\begin{tabular}{|c|c|c|c|c|c|}
\hline Patescibacteria & genus Candidatus Saccharimonas.1 & 0.000149 & 5.9624 & $0.0048 \pm 0.0010$ & $0.0008 \pm 0.0002$ \\
\hline Patescibacteria & genus Candidatus Saccharimonas & 0.000333 & 3.8575 & $0.0083 \pm 0.0014$ & $0.0021 \pm 0.0004$ \\
\hline Actinobacteria & genus Bifidobacterium & 0.000365 & 0.009988 & $1.70 \mathrm{E}-06 \pm 1.23 \mathrm{E}-06$ & $0.0004 \pm 0.0001$ \\
\hline Firmicutes & family Erysipelotrichaceae. 1 & 0.000381 & 9.0356 & $0.0002 \pm 5.18 \mathrm{E}-05$ & 2.79E-05 $\pm 8.53 \mathrm{E}-06$ \\
\hline Bacteroidetes & genus Parabacteroides & 0.000696 & 2.5102 & $0.0010 \pm 0.0001$ & $0.0003 \pm 7.70 \mathrm{E}-05$ \\
\hline Firmicutes & genus Ruminococcaceae UCG-014 & 0.000775 & 3.8747 & $0.0013 \pm 0.0003$ & $0.0003 \pm 0.0002$ \\
\hline Firmicutes & $\begin{array}{l}\text { genus Ruminococcaceae UCG- } \\
010.2\end{array}$ & 0.000775 & 2.1063 & $0.0004 \pm 4.25 \mathrm{E}-05$ & $0.0002 \pm 2.73 \mathrm{E}-05$ \\
\hline Bacteroidetes & genus Prevotella 9 & 0.000803 & 3.2998 & $0.0086 \pm 0.0011$ & $0.0025 \pm 0.0007$ \\
\hline Firmicutes & Lactobacillus gasseri & 0.000849 & 0.14097 & $9.59 \mathrm{E}-05 \pm 3.80 \mathrm{E}-05$ & $0.0007 \pm 0.0002$ \\
\hline Tenericutes & genus Anaeroplasma & NA & NA & $0.0017 \pm 0.0006$ & ND \\
\hline Firmicutes & genus Blautia.2 & NA & NA & ND & $6.10 \mathrm{E}-05 \pm 2.38 \mathrm{E}-05$ \\
\hline Firmicutes & genus Ruminiclostridium & 0.00122 & 0.012429 & $4.25 \mathrm{E}-06 \pm 1.99 \mathrm{E}-06$ & $0.0005 \pm 0.0002$ \\
\hline Firmicutes & family Christensenellaceae.1 & 0.002353 & 2.6456 & $0.0002 \pm 2.48 \mathrm{E}-05$ & 7.57E-05 $\pm 1.75 \mathrm{E}-05$ \\
\hline Firmicutes & genus Tyzzerella 3 & NA & NA & $0.0001 \pm 6.01 \mathrm{E}-05$ & ND \\
\hline Firmicutes & $\begin{array}{l}\text { genus Lachnospiraceae NK4A136 } \\
\text { group. } 2\end{array}$ & 0.002871 & 0.30295 & $1.75 \mathrm{E}-05 \pm 7.60 \mathrm{E}-06$ & $0.0087 \pm 0.0012$ \\
\hline Firmicutes & genus Oscillibacter.1 & 0.002998 & 0.24738 & $0.0019 \pm 0.0004$ & $0.0080 \pm 0.0017$ \\
\hline Proteobacteria & genus Oxalobacter & 0.004546 & 2.794 & $0.0002 \pm 2.87 \mathrm{E}-05$ & 4.84E-05 $\pm 1.58 \mathrm{E}-05$ \\
\hline Firmicutes & genus Lachnospiraceae UCG-010 & 0.005379 & 8.7386 & $0.0001 \pm 3.30 \mathrm{E}-05$ & $1.13 \mathrm{E}-05 \pm 4.30 \mathrm{E}-06$ \\
\hline Firmicutes & genus Lachnoclostridium.1 & 0.005824 & 5.1709 & $0.0033 \pm 0.0009$ & $0.0007 \pm 0.0002$ \\
\hline
\end{tabular}




\begin{tabular}{|l|l|c|c|c|c|}
\hline Firmicutes & family Clostridiales vadinBB60 & 0.006673 & 0.44348 & $0.0014 \pm 0.0003$ & $0.0033 \pm 0.0005$ \\
\hline Firmicutes & genus Intestinimonas.1 & 0.011292 & 0.21214 & $5.41 \mathrm{E}-05 \pm 1.22 \mathrm{E}-05$ & $0.0002 \pm 6.11 \mathrm{E}-05$ \\
\hline Firmicutes & genus Harryflintia.1 & 0.011569 & 0.27058 & $2.64 \mathrm{E}-05 \pm 8.10 \mathrm{E}-06$ & $9.83 \mathrm{E}-05 \pm 2.50 \mathrm{E}-05$ \\
\hline Firmicutes & family Lachnospiraceae.4 & 0.012257 & 3.6923 & $0.0015 \pm 0.0004$ & $0.0004 \pm 7.30 \mathrm{E}-05$ \\
\hline Firmicutes & genus Ruminococcus 1 & 0.013196 & 0.22256 & $6.74 \mathrm{E}-05 \pm 1.39 \mathrm{E}-05$ & $0.0003 \pm 8.96 \mathrm{E}-05$ \\
\hline Firmicutes & genus Tyzzerella & NA & NA & ND & $8.56 \mathrm{E}-06 \pm 2.78 \mathrm{E}-06$ \\
\hline Firmicutes & family Ruminococcaceae & 0.021464 & 0.3296 & $2.09 \mathrm{E}-05 \pm 5.34 \mathrm{E}-06$ & $5.70 \mathrm{E}-05 \pm 1.11 \mathrm{E}-05$ \\
\hline Firmicutes & genus Marvinbryantia & 0.022038 & 0.22811 & $6.54 \mathrm{E}-05 \pm 1.38 \mathrm{E}-05$ & $0.0003 \pm 7.89 \mathrm{E}-05$ \\
\hline Firmicutes & genus Acetatifactor & 0.023512 & 7.8109 & $0.0005 \pm 0.0002$ & $6.58 \mathrm{E}-05 \pm 1.78 \mathrm{E}-05$ \\
\hline Firmicutes & family Erysipelotrichaceae & 0.044132 & 2.691 & $0.0007 \pm 0.0002$ & $0.0003 \pm 4.32 \mathrm{E}-05$ \\
\hline
\end{tabular}

$\exists$ ND: Relative abundance was below detectable limits by $16 \mathrm{~S}$ sequencing

NA: Statistical comparison was not applicable due to a relative abundance below detectable limits (ND) in at least one GM group 


\section{CHAPTER V}

Conclusions and Future Directions 
Despite decades of research on both initiating factors and therapeutic approaches in colorectal cancer, CRC remains one of the leading causes of cancer-related morbidity and mortality. The breadth of CRC research to date speaks to the multifactorial etiologies of sporadic disease, and necessitates dedication to a variety of research avenues including genetic predisposition, dietary and environmental factors, and pre-disposing conditions among others. Epidemiological studies have described various associations between the aforementioned factors and CRC risk across human populations. However, human studies are limited in their capacity to address underlying mechanisms driving these associations, leading to the revolutionary use of animal models to distinguish causative factors. Though various models of CRC exist, we opted to use the $A p c^{\text {Min }}$ mouse model of early intestinal neoplasia in the outlined body of work due to its well-characterized genetics that includes known disease modifying variants, its quantitative adenoma phenotype, and established phenotype variability across different colonies.

Meta-analysis of published Min datasets reveals significant variability of unknown origin with respect to the adenoma phenotype between distinct colonies. These disparities reflect our minimal understanding of how various complex factors collectively determine overall adenoma susceptibility, and further highlight the challenges of enhancing reproducibility in our animal models. In the described body of work, we aimed to better understand how genetic variation and the complex gut microbiota (GM) each contribute to adenoma initiation in Min mice, and thus account for observed phenotypic variability (chapter 2). To follow, we used Min mice with a defined complex GM as a platform to interrogate the influence of individual bacterial species on adenoma initiation and development (chapter 3, Appendix A). Finally, we assessed the phenotypic effects of 
related substrain genetic backgrounds, and further evaluated whether the complex GM influences substrain-mediated adenoma suppression (chapter 4).

In chapter 2, we used Complex Microbiota Targeted Rederivation (CMTR) to determine how genetic variation acquired through colony divergence, and distinct complex GMs representing two common mouse producers (GMJAX and GMHSD), each contribute to Min adenoma multiplicity. We found that genetic lineage and complex GM additively conferred overall adenoma multiplicity, supporting the multifactorial nature of CRC. Whole-genome sequencing (WGS) and untargeted metabolomics were used to characterize genetic divergence and to identify differentially modulated pathways between the two lineages. Notably, each approach independently identified genetic modulation of bile acid (BA) metabolism, suggesting that metabolomics could be used as a tool to discern functionally relevant variants detected by WGS. To that end, we found that BA metabolism changes were associated with an upstream variant of Fatty acid binding protein 6 (Fabp6), which encodes for a BA transporter in the small intestinal (SI) epithelium. Moreover, segregation of the Fabp6 variant correlated with adenoma number in N2 cohorts generated from the divergent lineages, suggesting the variant's potential role as a modifier of Min.

Interestingly, we noted that several outliers were present in the N2 cohorts despite an overall association between the Fabp6 variant and adenoma multiplicity. This raises the possibility that the true modifier variant is not Fabp6 itself, but instead another variant linked to Fabp6 on chromosome 11. In such a case, the observed N2 outliers represent instances in which the unknown linked variant segregated from Fabp6 by recombination in the $\mathrm{N} 2$ generation. Thus, further studies interrogating the functionality 
of other WGS-detected variants on chromosome 11 are required to determine whether an unknown linked variant mediates the observed modifying effect.

Finally, we used $16 \mathrm{~S}$ rRNA sequencing to determine the relative abundances of all detected OTUs and thereby characterize community differences between adenomasuppressing GMJAX and adenoma-enhancing GMHSD. We determined that GMHSD had significantly increased overall richness and $\alpha$-diversity compared to GMJAX, indicating the relative difference in complexity between the two communities. Moreover, sulfite-reducing Bilophila wadsworthia and mucin-degrading Akkermansia muciniphila were undetectable in the GMJAX community, while they were present at various abundances in GMHSD. Given these disparities between GMJAX and GMHSD at the individual OTU level, Min mice colonized with GMJAX serve as an ideal platform to test the effects of candidate bacteria in the context of a complex GM.

Despite these findings, a mechanistic understanding of how each community as a whole induces a differential adenoma phenotype is lacking. The immune system can promote cell growth and survival or tumor suppression, and is therefore intimately associated with cancer susceptibility (Carvalho, Pires et al. 2014). The commensal GM has a critical role in sustaining host mucosal immune development and responses, suggesting that distinct communities might predispose the host towards an anti- or protumorigenic immunophenotype. Various studies have demonstrated the anti-tumorigenic role of Th1 immune responses, whereas microbiota-mediated induction of mucosal Th17 responses are associated with enhanced tumorigenesis (Osawa, Nakajima et al. 2006, Wu, Rhee et al. 2009, Chae, Gibson et al. 2010). Thus, additional studies addressing differential host immune responses following colonization with GMJAX and GMHSD 
may provide important insight into underlying mechanisms driving GM-mediated differential adenoma multiplicity.

In chapter 3, we used Min mice colonized with GMJAX as a platform to determine how Bilophila wadsworthia affects the broader GM, the host metabolome, and adenoma development. We observed that maternal supplementation with $B$. wadsworthia successfully transferred to Min offspring, which notably resulted in the depletion of fecal and mucosal-associated Prevotella sp. These results suggest that B. wadsworthia and Prevotella sp. share an environmental niche within the GI tract and may competitively exclude one another. This observation further demonstrates the importance of incorporating models with complex GMs rather than solely relying on highly simplified microbiotas, as we would not have detected the inverse relationship between $B$. wadsworthia and Prevotella in simplified models. Despite our hypothesis that $B$. wadsworthia would enhance adenoma multiplicity, its supplementation unexpectedly reduced Min adenoma number and size. Moreover, $B$. wadsworthia supplementation modulated both local and systemic metabolism, as evidenced by changes in the fecal and serum metabolomes characterized by untargeted and targeted metabolomics. These changes were highlighted by increased fecal abundance of the secondary BA deoxycholic acid (DCA), and decreased serum abundance of DCA.

Though it is presently unclear how B. wadsworthia supplementation suppresses adenoma initiation and progression, microbiota-mediated host immune induction provides one plausible explanation. Previous studies have described the immunogenic nature of $B$. wadsworthia characterized by induction of classic anti-tumorigenic Th1 immune responses in the intestines, whereas Prevotella sp. induce pro-tumorigenic Th17- 
mediated inflammation in vitro and in vivo via associated cytokines such as IL-17, IL-23, and IL-1 (Devkota, Wang et al. 2012, Larsen 2017). Combining the aforementioned results with previously established studies, it would be of interest to determine whether there are differences in mucosal immunity between Min mice colonized with GMJAX and supplemented B. wadsworthia compared to those with GMJAX alone, with special focus on quantifying markers of Th1 and Th17 responses in the mucosa and local mesenteric lymph nodes. Furthermore, studies using complex GMs can be used in concert with gnotobiotic methods to facilitate our understanding of the relationship between $B$. wadsworthia and Prevotella sp. Using a simplified GM baseline, co-inoculation experiments incorporating the two candidate taxa may determine whether $B$. wadsworthia-mediated Min adenoma suppression is dependent on Prevotella sp. depletion. These studies would provide substantial mechanistic insight into microbiotamediated adenoma modulation, and demonstrate an ideal combination of complex GM and gnotobiotic approaches to enhance model translatability.

In addition to our experiments concerning the effects of $B$. wadsworthia, we also used Min mice colonized with GMJAX to determine whether Akkermansia muciniphila influences Min adenoma multiplicity (Appendix A). As we observed in our $B$. wadsworthia experiments, maternal supplementation resulted in successful maternal transfer of A. muciniphila to Min offspring. However, there were no changes in adenoma multiplicity associated with increased relative abundance of A. muciniphila, suggesting that this bacterium does not have a direct role in adenoma initiation. Interestingly, this experiment was complicated by significant morbidity and mortality among weanlings born from supplemented females, characterized by a failure to thrive. Further analysis 
demonstrated a marked increase in colonic goblet cells and mucus deposition on the colonic epithelial surface. Though it is presently unclear whether these changes are directly responsible for increased morbidity and mortality, these observations suggest that enrichment of A. muciniphila in neonates could have detrimental effects on growth and development. Given the recent push to include A. muciniphila as a probiotic to counteract obesity and metabolic disease (Cani and de Vos 2017), further studies are required to evaluate its potential negative effects in pregnant women and neonates.

In chapter 4, we asked whether the background genetics of related inbred mouse substrains, C57BL/6J (B6/J) and C57BL/6NHsd (B6/N) have differential modifier effects with respect to the Min phenotype. Of note, both B6/J and B6/N backgrounds dominantly repressed adenoma multiplicity. However, the $\mathrm{B} 6 / \mathrm{N}$ background was associated with stronger suppression of the phenotype compared to B6/J. Moreover, we used CMTR to demonstrate that the complex GM further modulated B6/N-mediated adenoma suppression, such that GMHSD again conferred greater adenoma susceptibility than GMJAX. These results suggest that the differences between $\mathrm{B} 6 / \mathrm{J}$ and $\mathrm{B} 6 / \mathrm{N}$ provide a platform for future identification of modifiers of Min. Moreover, these results should serve as a caution for investigators that use these substrains interchangeably. Here we demonstrate that these substrains have differential effects on the Min phenotype, but their influence on other commonly used models of complex disease traits remains unknown. Thus, strict control of background genetics and complex GM in disease models is required to enhance reproducibility and translatability to humans.

Collectively, the outlined body of work demonstrates the use of rapidly advancing technology to interrogate factors that influence the adenoma phenotype in the Min model 
of CRC. Based on the reported results, it is clear that we must pursue a variety of research avenues to gain a better understanding of the most important factors driving composite disease susceptibility. As precision medicine approaches to complex diseases continue to gain traction, incorporation of omics techniques that develop a broad understanding of an individual's risk for disease development and response to therapeutic strategies is critical. Thus, models in which GM profiling and genomic characterization are coupled with other omics strategies such as metabolomics, proteomics, metagenomics, and metatranscriptomics will pave the way for integrated approaches in humans that describe functional biological changes associated with diseases of interest. Moreover, pairing large-data analyses with follow-up mechanistic studies will further enable the development of specific therapeutic strategies that target the pathways elucidated by integrated omics. Although this work only begins to tap into the vast array of genetic and environmental variables that affect CRC, it provides a framework for novel approaches to a range of disease processes. In doing so, this work has the potential to enhance risk assessment, diagnostic and prognostic approaches, and patient-targeted therapeutic strategies for both CRC and other complex disease processes. 


\section{APPENDIX A: \\ Effects of Akkermansia muciniphila Supplementation on Min Adenoma Initiation}




\section{A.1. Overview}

Colorectal cancer (CRC) is a multifactorial disease with overall susceptibility determined by genetic and environmental etiologies (Lichtenstein, Holm et al. 2000, de la Chapelle 2004). The gut microbiota (GM) is a complex collection of bacterial species that normally exist in a symbiotic relationship with their host, and is shaped in part by environmental factors such as diet and geographical location (Lozupone, Stombaugh et al. 2012, Brim, Yooseph et al. 2013, Dejea, Wick et al. 2014). Culture-independent methods of bacterial identification have provided a wealth of knowledge about the beneficial roles of these communities, including protection from enteric pathogens, mucosal immune development, and nutrient extraction from dietary components (Lozupone, Stombaugh et al. 2012, Dingemanse, Belzer et al. 2015). However, disruption of the GM can contribute to various metabolic diseases, inflammatory conditions, and even promote tumor development (Backhed, Ding et al. 2004, Arthur and Jobin 2011). Therefore, studies evaluating how specific commensal strains contribute to health and disease will inform the use of the GM for risk assessment and novel therapeutic strategies.

Akkermansia muciniphila is a gram-negative, mucin-degrading species found in the intestinal mucus layer and feces of adults (Collado, Derrien et al. 2007). Due to the $A$. muciniphila's intimate association with the intestinal mucosa, its potential to mediate host-microbiota interactions that modulate disease susceptibility is of interest. To that end, studies focused on this strain's role in cross-talk with the host highlight $A$. muciniphila's protective role in metabolic shifts associated with diabetes and obesity. Everard et al. demonstrated that A. muciniphila administration counteracts diet-induced 
metabolic endotoxemia, fat mass development, and altered adipose tissue metabolism (Everard, Belzer et al. 2013), while others have associated A. muciniphila enrichment with the anti-diabetic effects of metformin (Shin, Lee et al. 2014). Based on these studies, many have suggested $A$. muciniphila as a next-generation probiotic to combat increasing prevalence of negative health effects associated with metabolic syndrome. While the aforementioned pre-clinical data is encouraging, studies that adequately explore possible negative effects of A. muciniphila enrichment in the GM are required to inform any risks associated with its use as a probiotic species. In contrast to apparent beneficial health effects, A. muciniphila was reportedly enriched approximately 4-fold in CRC patients compared to healthy controls (Weir, Manter et al. 2013). Presently, it is unclear whether this association reflects an underlying causative role of $A$. muciniphila in CRC development, or if the commensal expands as a bystander. Therefore, studies that specifically address the role of $A$. muciniphila in $\mathrm{CRC}$ will provide a broader understanding of potential risks associated with enriched A. muciniphila in the context of the complex GM.

The C57BL/6J-Apc $c^{\text {Min }}\left(A p c^{\text {Min }}\right)$ mouse has a dominant mutation in the $A p c$ gene resulting in the development of intestinal adenomas, which can be used as a quantitative phenotype to test genetic or environmental modifiers of CRC susceptibility (Shoemaker, Gould et al. 1997). Here, we use specific-pathogen-free (SPF) $A p c^{M i n}$ mice with no previously detectable $A$. muciniphila to explore how addition of the commensal strain to an existing complex GM affects adenoma initiation. We show that single treatment of pregnant dams results in highly efficient vertical transmission of A. muciniphila to Apc ${ }^{\text {Min }}$ offspring. Offspring of A. muciniphila-treated dams displayed significant differences in 
colonic development of the mucosal barrier system compared to controls. Moreover, these developmental changes dramatically influence the pups' ability to thrive during the neonatal period. However, there was no apparent effect of $A$. muciniphila supplementation on adenoma multiplicity in $A p c^{M i n}$ mice. These results suggest that although A. muciniphila does not appear to affect adenomagenesis in $A p c^{\text {Min }}$ mice, maternal transfer of high levels of this commensal may have significant health implications for neonates.

\section{A.2. Methods}

\section{A.2.1. Ethics statement}

Animal studies were conducted in an Association for Assessment and Accreditation of Laboratory Animal Care International (AAALAC) accredited facility based on the guidelines provided by the Guide for the Care and Use of Laboratory Animals. All animal studies were approved by the University of Missouri Institutional Animal Care and Use Committee.

\section{A.2.2. Animals}

Mice used in all described experiments were purchased from The Jackson Laboratory (Bar Harbor, ME), and were acclimated for several generations prior to use. Six to eight week old male C57BL/6J-Apc $c^{\mathrm{Min}} /+$ mice were mated with wild-type six to eight week old female C57BL/6J mice. After pregnancy was confirmed in mated females, they were individually housed and assigned to either a control (PBS) group or $A$. muciniphila group. At weaning (21 days of age), offspring of pregnant dams were grouphoused with sex-matched littermates within their experimental group. To genotype for the 
Min allele, all offspring were ear-punched at weaning, and DNA extracted using the "HotSHOT" genomic DNA preparation method as described as described (Truett, Heeger et al. 2000). Fecal samples were also collected from all Min offspring at weaning and stored at $-80^{\circ} \mathrm{C}$. Offspring from pregnant dams were separated into two cohorts. The first cohort was sacrificed at weaning for histological characterization and A. muciniphila detection, while the second cohort was sacrificed at 3 months of age for adenoma phenotyping. All experimental animals were housed in micro-isolator cages on ventilated racks (Thoren, Hazelton, PA) with ad libitum access to 5058 irradiated breeder chow (LabDiet, St. Louis, MO) and acidified autoclaved water. Mice were maintained on paper chip bedding (Shepherd Specialty Papers, Watertown, TN) with a 14:10 light:dark cycle.

\section{A.2.3. Akkermansia muciniphila culture and treatment}

Akkermansia muciniphila was purchased from American Type Culture Collection (ATCC BAA-835; Manassas, VA), grown in Brain Heart Infusion (BHI) broth (Anaerobe Systems, Morgan Hill, CA), then flash-frozen in glycerol solution (25\% v/v glycerol) and stored at $-80^{\circ} \mathrm{C}$. For treatment, $1 \mathrm{~mL}$ of frozen stock was thawed, inoculated into $10 \mathrm{~mL}$ $\mathrm{BHI}$ broth medium, and incubated at $37^{\circ} \mathrm{C}$ in an anaerobic chamber. When cultures reached late log-phase of growth, bacteria was pelleted by centrifugation and washed twice with 1x phosphate-buffered saline (PBS) pH 7.3 (Ausubel FM 1994). The pellet was resuspended in PBS to a final concentration of $1.0 \times 10^{7} \mathrm{CFUs} / 250 \mathrm{uL}$ PBS. Intragastric gavage was used for a single treatment of dams in the final week of pregnancy with either 250uL PBS (control group), or 250uL PBS containing 1.0x107 CFUs $A$. muciniphila (A.m. group).

\section{A.2.4. DNA extraction for qPCR analysis}


Fecal samples and SI contents were mechanically disrupted using a TissueLyser II (Qiagen, Venlo, Netherlands) for 3 minutes at $45 \mathrm{~Hz}$, then incubated at $70^{\circ} \mathrm{C}$ for 20 minutes with periodic vortexing. DNA extraction from fecal pellets for 16S rRNA sequencing was performed using the DNeasy Blood \& Tissue Kit ${ }^{(Q i a g e n)}$ as previously described (Ericsson, Davis et al. 2015).

\section{A.2.5. Akkermansia muciniphila colonization}

Fecal samples and SI contents were collected from cohort 1 upon sacrifice at weaning to test for colonization of A. muciniphila by quantitative PCR (qPCR) analysis. A muciniphila quantification is expressed as copy number relative to $16 \mathrm{~S}$ eubacterial copy number for an estimate of relative abundance. Each $10 \mathrm{uL}$ reaction contained $5 \mathrm{uL}$ of 2x iTaq ${ }^{\mathrm{TM}}$ Universal SYBR ${ }^{\circledR}$ Green Supermix (Bio-Rad, Hercules, CA), $2 \mathrm{uL}$ of $2 \mathrm{uM}$ forward and reverse primers (final concentration $400 \mathrm{nM}$ ), and $1 \mathrm{ng}$ DNA template. The following PCR parameters were used: initial incubation at $95^{\circ} \mathrm{C}$ for 10 minutes, denaturation at $95^{\circ} \mathrm{C}$ for 15 seconds, annealing and elongation at $60^{\circ} \mathrm{C}$ for 1 minute for a total of 45 cycles. Previously validated primers were used to detect A. mисiniphila and universal 16S rRNA as described (Alam, Leoni et al. 2016), and all reactions were performed in triplicate. Standard curves were generated using DNA extracted from pure A. muciniphila culture using both A. muciniphila primers and 16S rRNA eubacterial primers. Standard curves were then used to estimate copy numbers of A. muciniphila and 16S rRNA in fecal and SI samples.

\section{A.2.6. Histological quantification of goblet cells}

At weaning age (21 days), $A p c^{M i n}$ mice in cohort 1 were sacrificed by $\mathrm{CO} 2$ asphyxiation. The abdominal cavity was opened and the entire gastrointestinal tract was 
removed. An approximately $1 \mathrm{~cm}$ length of terminal ileum and proximal colon was removed from each animal's GI tract for histological sectioning. Tissue sections were immersed in 10\% buffered formalin for 24 hours and processed using Alcian Blue (final pH 2.5) stain with a Periodic Acid-Schiff (PAS) counterstain to visualize sulfated and carboxylated sialomucins (Takeyama, Fahy et al. 2001). For goblet cell (GC) quantification, GCs and total nuclei were counted in five colonic and five ileal crypts in each animal, then averaged. These values were used to determine a colonic and SI GC:nucleus ratio in each mouse. GC and nuclei counts, and histological imaging were performed using a Zeiss Axiophot microscope (Oberkochen, Germany) with the Olympus DP70 imaging system (Tokyo, Japan).

\section{A.2.7. Adenoma multiplicity}

All $A p c^{M i n}$ offspring in cohort 2 were sacrificed 3 months of age via $\mathrm{CO}_{2}$ asphyxiation for adenoma phenotyping. Following exposure through the abdominal cavity, whole SI and colons were removed and opened longitudinally, then placed on bibulous paper with the luminal side facing up. The luminal surface was flushed with saline and formalin-fixed. Grossly visible adenomas were counted manually using a Leica M165FC microscope (Leica, Buffalo Grove, IL) at 1.25x magnification.

\section{A.2.8. Statistical analysis}

Statistical analyses for qPCR determination of A. muciniphila colonization, GC quantification, and adenoma multiplicity were performed with Sigma Plot 14.0 (Systat Software Inc., Carlsbad CA). The Mann-Whitney rank sum test was used to compare relative abundance A. muciniphila between control and A.m. groups, and the student's ttest was used for statistical comparisons GC:nucleus ratios and adenoma multiplicity. 
For all statistical tests, $p<0.05$ was considered significant. GraphPad Prism 8 was used to generate all scatter plots and bar graphs.

\section{A.3. Results}

\section{A.3.1. Akkermansia muciniphila demonstrates maternal transmission to Min offspring}

To determine the effects of $A$. muciniphila on adenoma multiplicity, we aimed to colonize neonatal $A p c^{M i n}$ mice with the bacterium. To do so, we used mice from a colony with no previously detected A. muciniphila, and opted to supplement pregnant dams such that experimental $A p c^{\text {Min }}$ offspring would acquire it immediately upon birth. Pregnant dams were assigned to either a PBS (control) group or PBS + A. muciniphila (A.m.) group. In the final week of pregnancy, controls were treated with PBS while the A.m. group was treated with $1.0 \times 10^{7} \mathrm{CFUs}$ A. muciniphila by gastric gavage. Following treatment, qPCR analysis of fecal samples was used to monitor maternal relative abundance of A. muciniphila. (Fig. 1A). Apc ${ }^{\text {Min }}$ offspring of control and A.m. pregnant dams were separated into two cohorts, one of which was sacrificed at weaning for evaluation A. muciniphila colonization in SI contents and feces and histological characterization. In both SI and feces samples, $A p c^{\text {Min }}$ pups from A.m. dams had significantly increased relative abundances of $A$. muciniphila compared to pups from control dams (Fig. 1B). Thus, maternal supplementation resulted in successful colonization of $A p c^{M i n}$ mice with $A$. muciniphila by vertical transmission.

\section{A.3.2. Akkermansia muciniphila modulates colonic goblet cells and mucin secretion}


After successful colonization of $A p c^{\text {Min }}$ mice with A. muciniphila we sought to characterize early intestinal changes that may coincide, and potentially influence adenoma initiation. A. muciniphila degrades mucins as a source of carbon and nitrogen, and therefore localizes close to the intestinal epithelium for access to mucins secreted by goblet cells. We asked whether the presence of $A$. muciniphila modulates GCs within the SI and colonic epithelium, and whether there are differences in mucin secretion between control and A.m. mice. Histological sections of SI and colon were stained with Alcian Blue to visualize GCs and secreted carboxylated and sulfated sialomucins. Representative images of stained histological sections demonstrate that while control mice have relatively few GCs with very little apparent mucin staining on the epithelial surface, A.m. mice harbor abundant distinct GCs (red arrow) with significant mucus blebbing (black arrow) (Fig. 2A). To follow, GCs in the terminal ileum and proximal colon were quantified as a ratio to total nuclei per crypt. GC quantification revealed that A.m. mice had significantly more colonic GCs per crypt compared to control mice (Fig. 2B), while this effect was not observed in the terminal ileum (Fig. S1). These results indicate that the presence of maternally transmitted A. muciniphila alters colonic GC proliferation and mucin secretion.

\section{A.3.3. Akkermansia muciniphila colonization does not influence Min adenoma initiation}

Having established significant physiological effects of A. muciniphila, we evaluated whether Min adenoma initiation was altered in A.m. Apc ${ }^{\text {Min }}$ mice. To address this, a second cohort of control and A.m. Apc ${ }^{M i n}$ mice was sacrificed at 3 months of age for SI and colonic adenoma phenotyping. We used adenoma multiplicity to estimate 
overall susceptibility to adenoma initiation, and therefore manually counted all visible adenomas. We found no significant differences in either colonic or SI adenoma multiplicity between controls and A.m (Fig. 3), indicating that there was no apparent effect of $A$. muciniphila on adenoma formation in Min mice.

\section{A.4. Discussion}

Recent studies have established the GM as an important component of the multifactorial etiology of CRC (Ericsson, Akter et al. 2015, Chen and Vitetta 2018, Mendes, Paulino et al. 2018). In a previous study (Chapter 2, supplementary table 4), we identified enrichment of Akkermansia sp. in a complex GM community associated with increased adenoma multiplicity in $A p c^{M i n}$ mice. Here, we used $A p c^{M i n}$ to determine whether Akkermansia muciniphila has a causative role in increased adenoma initiation. We inoculated pregnant dams with A. muciniphila, which successfully transferred to $A p c^{M i n}$ offspring such that they were heavily colonized by weaning age. We show that $A$. muciniphila supplementation modulates colonic GC proliferation and mucin secretion, and further show that A. muciniphila does not affect adenoma multiplicity in the $A p c^{\text {Min }}$ mouse model. Together, this data demonstrates that while there is no evidence of a causative link between A. muciniphila and adenoma initiation, this microbe does have significant potential to mediate health through established physiological effects.

Vertical transmission of the GM from mother to newborn is thought to initiate host-microbe symbiosis which has an important role in early intestinal development and lifelong health, but little is known about maternal transfer of specific commensal strains such as A. muciniphila (Korpela, Costea et al. 2018). We found that when pregnant 
female mice were inoculated with A. muciniphila, there was highly efficient transfer of the commensal bacteria to $A p c^{M i n}$ offspring (Fig. 1). Interestingly, one study focused on mothers and newborn infants suggested variable vertical transmission of A. muciniphila, and a delayed enrichment of A. muciniphila in those that did acquire it (Korpela, Costea et al. 2018). This disparity may be explained by the mode of maternal acquisition of $A$. muciniphila. While Korpela et al. traced commensals that were endogenously present in the maternal microflora, our study models introduction of the bacteria in previously naive late-term pregnant dams. Thus, efficient vertical transmission may be due to higher relative abundances of A. muciniphila within the GI tract and more active shedding of the bacteria in feces. Thus, our approach more closely models novel exposure to $A$. muciniphila by means of probiotics or dietary changes that promote its expansion in the GI tract (Desai, Seekatz et al. 2016).

A. Muciniphila degrades mucins via breakdown of $\mathrm{N}$-acetylglucosamine and $\mathrm{N}$ acetylgalactosamine as a source of carbon and nitrogen, and is therefore intimately associated with the intestinal mucosa (Collado, Derrien et al. 2007, Derrien, Collado et al. 2008). Thus, we hypothesized that histological analysis of weanling $A p c^{M i n}$ mice from A.m.-treated dams would reveal a diminished mucus layer on SI and colonic epithelial surfaces relative to controls. In fact, we observed that the opposite was true, such that A.m. mice had abundant mucus "blebs" on the colonic epithelial surface, while this was not observed in controls (Fig. 2A). Moreover, these histological changes were associated with significantly increased colonic GC density in A.m. mice compared to controls (Fig. 2B). Although the underlying cause for this observation is not clear, it is possible that $A$. muciniphila's mucin-degrading activity induces compensatory proliferation of GCs and 
subsequent mucin secretion. Ganesh et al. assessed whether A. muciniphila supplementation induces increased expression of $M u c 2$, which encodes for the primary mucin in the intestinal mucus layer, but found that was not that case (van der Lugt, van Beek et al. 2019). Thus, further studies are required to determine how A. muciniphila induces GC proliferation and mucin secretion.

Of note, we observed pup morbidity and mortality over the course of this experiment, which was limited to offspring of A.m.-treated dams. This was characterized by a failure to thrive at approximately 2 weeks of age, necessitating humane euthanasia of several pups in the A.m. group. Failure to thrive was observed in both $A p c^{M i n}$ offspring and their wild-type siblings, indicating that this phenomenon was not dependent on an interaction between the Min allele and A. mисiniphila. Given the changes in GC density and mucin secretion associated with A. muciniphila, it is possible that altered architecture of the mucus layer interfered with normal nutrient absorption in pups. In this case, novel introduction of high levels of A. muciniphila in pregnant women could pose a significant health threat to newborn infants. A. muciniphila has recently been proposed as a nextgeneration probiotic due to its benefits with respect to obesity and other metabolic diseases (Cani and de Vos 2017), and could therefore become a source of A. muciniphila enrichment. Additionally, recent studies show that shifts towards a low-fiber diet in mice promotes significant expansion of A. muciniphila (Desai, Seekatz et al. 2016). Based on our preliminary results indicating a failure to thrive in A.m. mice, factors that promote expansion of A. muciniphila in pregnant females and potentially harmful sequelae in neonatal infants due to vertical transfer should be thoroughly investigated. 
Finally, we evaluated whether intestinal changes associated with maternal transmission of A. muciniphila affected adenoma initiation in genetically-susceptible $A p c^{M i n}$ mice. We found that there were no significant differences in SI or colonic adenoma multiplicity between A.m. and control mice. Interestingly, these results contrast with previously reported work demonstrating increased adenoma multiplicity in Apc mutant mice following A. muciniphila supplementation (Dingemanse, Belzer et al. 2015). In this study, the authors treated mice with A. muciniphila at approximately 3-4 weeks of age, while our mice were colonized via natural transmission at birth. The disparate outcomes across these two studies suggest that timing of inoculation may be of critical importance to A. muciniphila's effects on adenomagenesis. It is possible that delayed introduction to the novel bacteria results in a pro-tumorigenic immune response (Derrien, Van Baarlen et al. 2011), whereas natural inheritance of A. muciniphila confers tolerance. Moreover, Dingemanse et al. found that co-inoculating Apc mutants with A. muciniphila and Helicobacter typhlonius resulted in reduced adenoma multiplicity compared to controls, suggesting that the surrounding intestinal communities dictate how $A$. muciniphila affects adenoma initiation. Thus, further studies examining how $A$. muciniphila interacts with the broader GM are necessary for a better understanding of the commensal's pathogenic potential.

As evidenced by these results, A. muciniphila can substantially affect intestinal physiology and development in neonatal mice. Here, we confirm that maternal transmission of supplemented A. muciniphila results in distinct changes in colonic GC density and mucus barrier composition. These observations coincided with pup morbidity and mortality prior to weaning due to a failure to thrive, suggesting a possible nutrient 
absorption deficiency. Finally, A. muciniphila supplementation had no apparent effect on adenoma formation in genetically susceptible $A p c^{M i n}$ mice. With the proposed use of $A$. muciniphila as a probiotic to combat a plethora of metabolic diseases, further studies addressing the safety of high levels of this commensal in pregnant women with respect to neonatal infants are warranted. Moreover, studies focused on how timing of introduction to specific commensal strains, as well as the complex interactions between various species, will further enhance our understanding of microbial etiologies in CRC.

\section{A.5. Financial Support}

This research was funded by grants from the National Institutes of Health to the Mutant Mouse Resource and Research Center at the University of Missouri (U42 OD010918), and by the University of Missouri to Dr. James Amos-Landgraf (Startup-funding). JEM was supported by NIH T32 OD011126. 


\section{A.6. Figures}

Figure 1. Maternal transmission of supplemented A. muciniphila. A, Dams in the final week of pregnancy after mating with C57BL/6J-Apc ${ }^{\text {Min }}$ mice were supplemented with PBS (control, $\mathrm{n}=3$ ) or $10^{7}$ CFUs A. muciniphila suspended in PBS $(A . m ., \mathrm{n}=4)$ via gastric gavage. Time-points shown on the $\mathrm{x}$-axis are relative to the date of treatment. Bar graphs represent quantitative PCR analysis of fecal samples for A. muciniphila copy number relative to $16 \mathrm{~S}$ rRNA gene copy number to assess mean ( \pm SEM) relative abundance of $A$. muciniphila in controls and treated mice. $\mathbf{B}$, Bar graphs comparing mean $( \pm \mathrm{SEM})$ relative abundance of $A$. muciniphila in SI contents and feces of $A p c^{M i n}$ offspring (control, $\mathrm{n}=15 ;$ A.m., $\mathrm{n}=15$ ) at weaning. $* * * p<0.001$; Mann-whitney rank sum test.

A

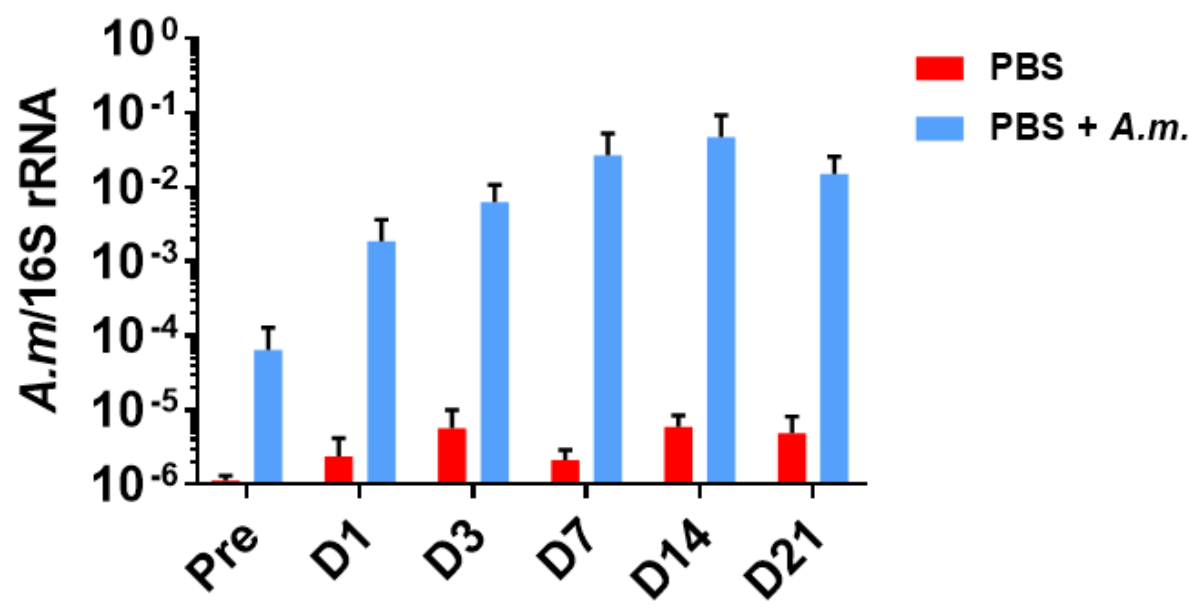


B

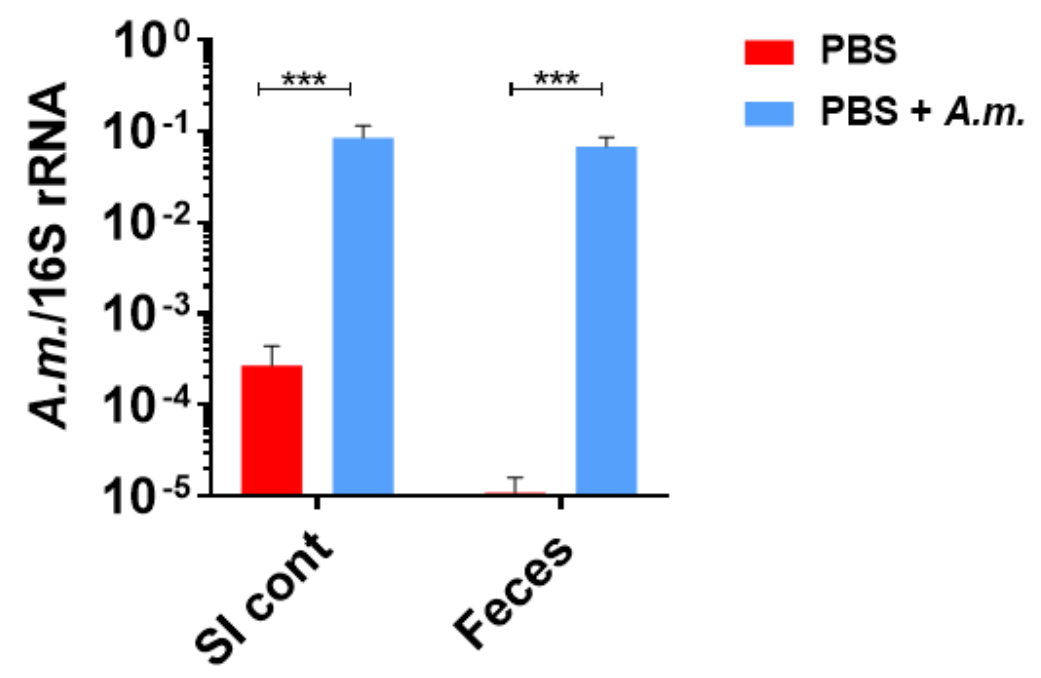


Figure 2. A. muciniphila supplementation effects on colonic goblet cells. A,

Representative images of colonic mucosa in weaning-aged $A p c^{M i n}$ offspring from dams supplemented with PBS (control, left) or PBS + A. muciniphila (A.m., right). Tissue sections were stained with alcian blue $(\mathrm{pH} 2.5)$ and PAS counterstain. Black arrow shows secreted mucins, and red arrow shows a mucin-filled goblet cell. 200X magnification. B, Scatter plot comparing mean $( \pm \mathrm{SD}$ ) colonic (control, $\mathrm{n}=15 ;$ A.m., $\mathrm{n}=15$ ) goblet cell to nucleus ratio. Each data-point represents the average GC:nucleus ratio using five crypts from an individual mouse. $* * * p<0.001$; Student's t-test.

A
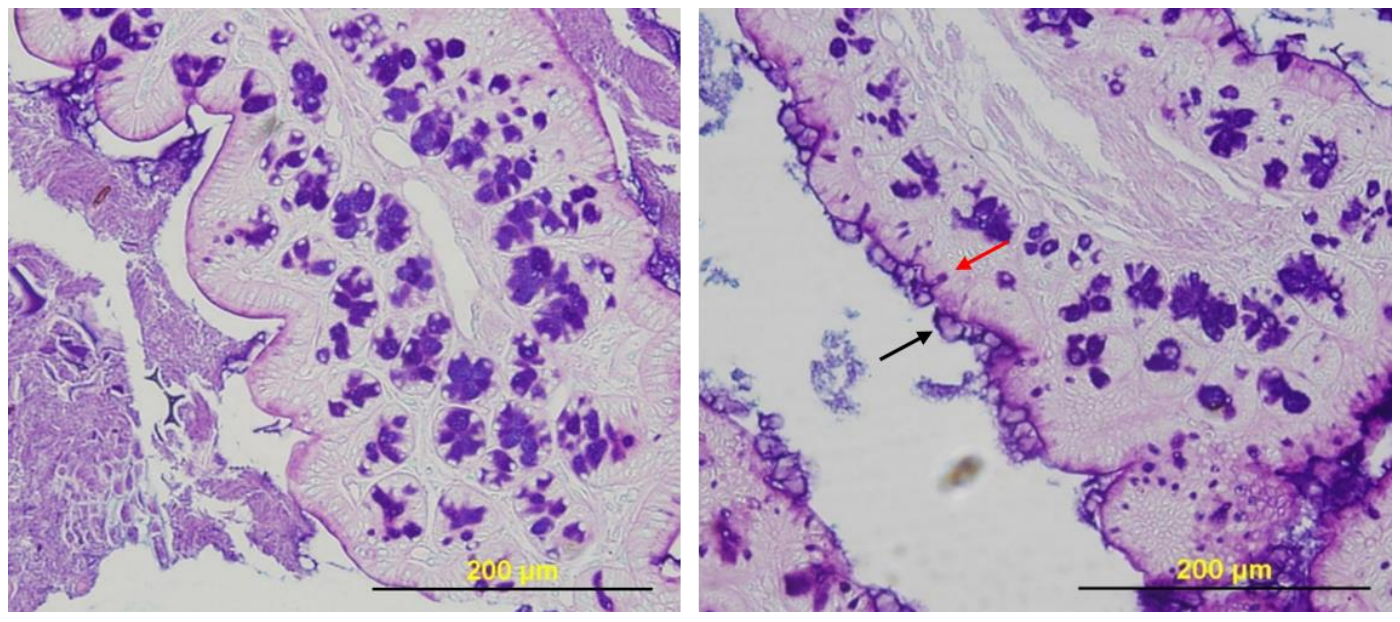
B

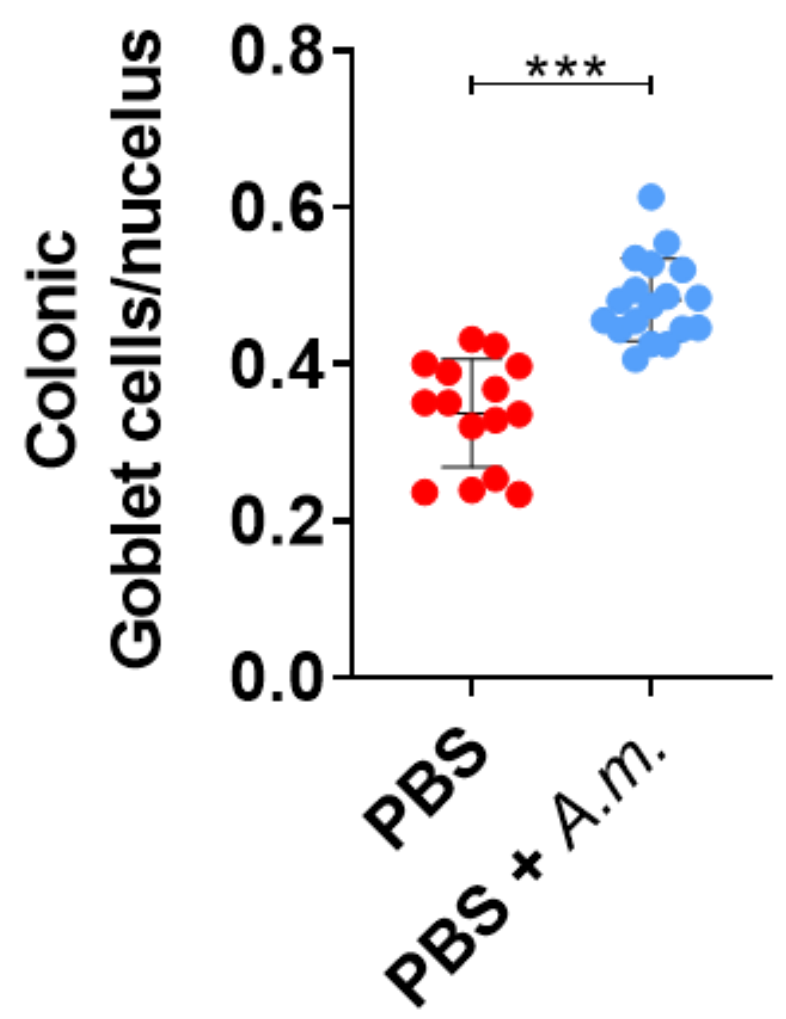


Figure 3. A. muciniphila supplementation effects on adenoma multiplicity. Scatter plots comparing mean $( \pm \mathrm{SD})$ colon $(\mathbf{A})$ and SI $(\mathbf{B})$ adenoma numbers in $A p c^{M i n}$ offspring (control, $\mathrm{n}=6$, A.m., $\mathrm{n}=6$ ) after sacrifice at 3 months of age. Student's t-test.

A

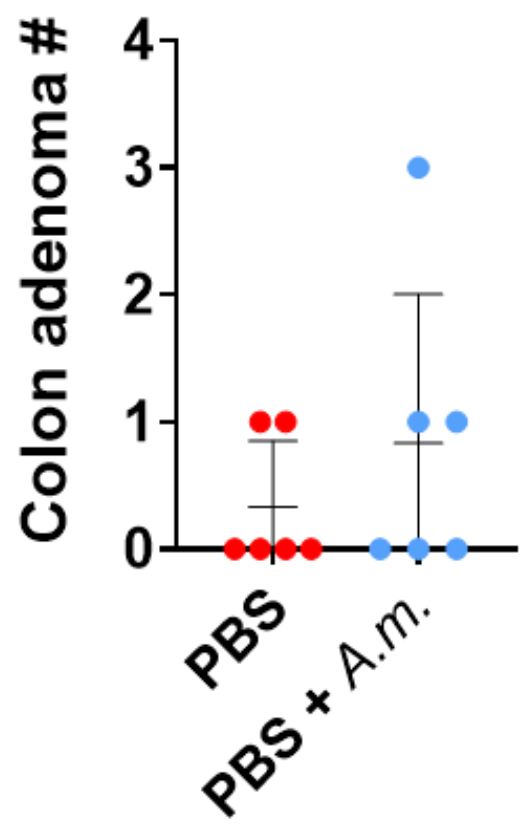

B

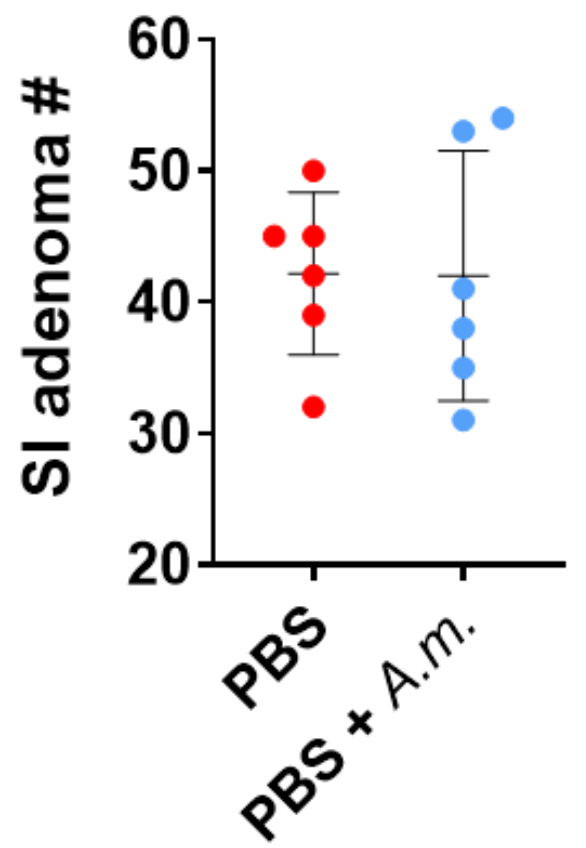




\section{Supplementary figure 1. A. muciniphila supplementation effects on SI goblet cells.}

A, Representative images of the ileal mucosa in weaning-aged $A p c^{M i n}$ offspring from dams supplemented with PBS (control, left) or PBS + A. muciniphila (A.m., right). Tissue sections were stained with alcian blue $(\mathrm{pH} 2.5)$ and PAS counterstain. The red arrow shows a mucin-filled goblet cell. 200X magnification. B, Scatter plot comparing mean ( \pm $\mathrm{SD}$ ) colonic (control, $\mathrm{n}=6 ;$ A.m., $\mathrm{n}=9$ ) goblet cell to nucleus ratio. Each data-point represents the average GC:nucleus ratio using five crypts from an individual mouse. Student's t-test.

\section{A}
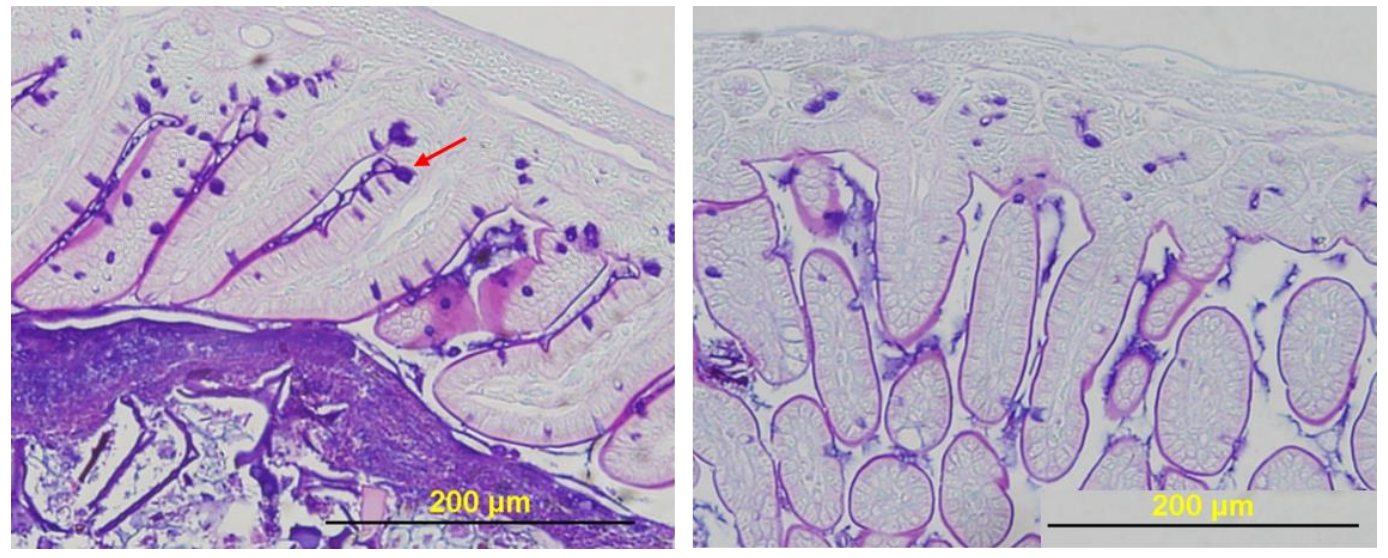
B

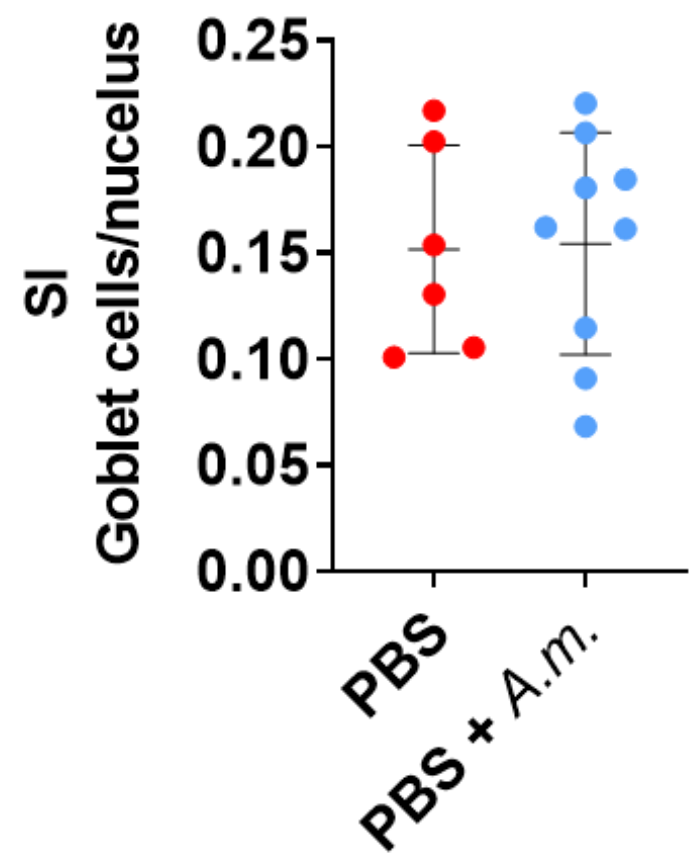




\section{APPENDIX B:}

Cross fostering as a Method of Complex Microbiota Transfer 


\section{B.1. Overview}

As investigators continue to develop interest the GM and how it affects model phenotypes, a strong understanding of different techniques used for microbiota manipulation is critical. Gnotobiotic facilities were established to enable the use of germfree mice, as well as mice with a simplified GM. However, there are a number of different approaches, each with advantages and disadvantages, that facilitate the transfer of complex GM communities. Some of the commonly used techniques for complex GM transfer include embryo transfer, fecal transplant, co-housing, and cross fostering (CF) (Hart, Ericsson et al. 2018). Hart et. al. developed colonies of CD1 outbred mice that harbored distinct complex GMs with the goal of using females within these colonies as GM donors for mouse models of interest. They demonstrated the utility of these colonies by transferring embryos harvested from an isogenic $1110^{-/-}$colony into CD1 surrogates harboring distinct complex GMs (Complex Microbiota Targeted Rederivation), and observed differential phenotype severity based on complex GM (Hart, Ericsson et al. 2017). We have also described the use of two of these colonies (GMJAX and GMHSD) to show that complex GM influences the Min adenoma phenotype (Chapter 2). Although rederivation yields efficient maternal microbiota transfer (Franklin and Ericsson 2017), this technique is relatively expensive and requires significant expertise for successful embryo transfer. Thus, studies are required to determine the effectiveness of other methods of GM transfer. Here, we test whether CF results in successful transfer of the GM from the aforementioned CD1 surrogates to B6/J offspring. Moreover, we use both GMJAX and GMHSD B6/J recipients and CD1 donors to determine whether the richness and diversity of the recipient and donor influences effectiveness of microbiota transfer. 


\section{B.2. Methods}

B6/J mice colonized with either GMJAX or GMHSD, as described in chapter 2, were used as recipients for $\mathrm{CF}$. Timed matings were set up such that $\mathrm{B} 6 / \mathrm{J}_{\mathrm{GMJAX}}$ dams would have pups at the same time as $\mathrm{CD} 1_{\mathrm{GMHSD}}$ surrogates, and $\mathrm{B} 6 / \mathrm{J}_{\mathrm{GMHSD}}$ dams would have pups at the same time as CD1 $1_{\mathrm{GMJAX}}$ surrogates. Within 12 hours of birth, B6/J $\mathrm{GMJAX}$ neonatal mice were removed from their cage, and fostered onto $\mathrm{CD} 1_{\mathrm{GMHSD}}$ surrogates in order to transfer GMHSD to the $\mathrm{B} 6 / \mathrm{J}_{\mathrm{GMJAx}}$ pups. Similarly, B6/J $\mathrm{J}_{\mathrm{GMHSD}}$ neonatal mice were fostered onto $\mathrm{CD} 1_{\mathrm{GMJAX}}$ surrogates to transfer GMJAX to the $\mathrm{B} 6 / \mathrm{J}_{\mathrm{GMHSD}}$ pups (Fig. 1). Fecal samples were collected from CF pups immediately after weaning and at 3 months of age, when all pups were humanely euthanized. DNA was extracted from all samples, and the microbial 16S rRNA genes were amplified, sequenced, and processed to determine the relative abundance of all detected OTUs. All DNA extraction, sequencing, and processing was performed as previously described in chapter 2, sections 2.5-2.6. Statistical analysis of richness, $\alpha$-diversity, and $\beta$-diversity were performed as described in chapter 2, section 2.12 . 


\section{B.3. Results and Figures}

Figure 1. Experimental design. C57BL/6J mice were previously colonized and maintained with either GMJAX or GMHSD via rederivation. B6/J pups born with the higher richness/diversity GMHSD (recipient, $\mathrm{n}=9$ ) were cross fostered to timed-mated CD1 GMJAX surrogate dams (donor) within 12 hours of birth, and B6/J pups born with the lower richness/diversity GMJAX (recipient, $\mathrm{n}=9$ ) with cross fostered to timed-mated $\mathrm{CD} 1_{\mathrm{GMHSD}}$ dams (donor) within 12 hours of birth. The surrogate dams were then allowed to nurse the foster pups in their cage until weaning at 3 weeks of age.

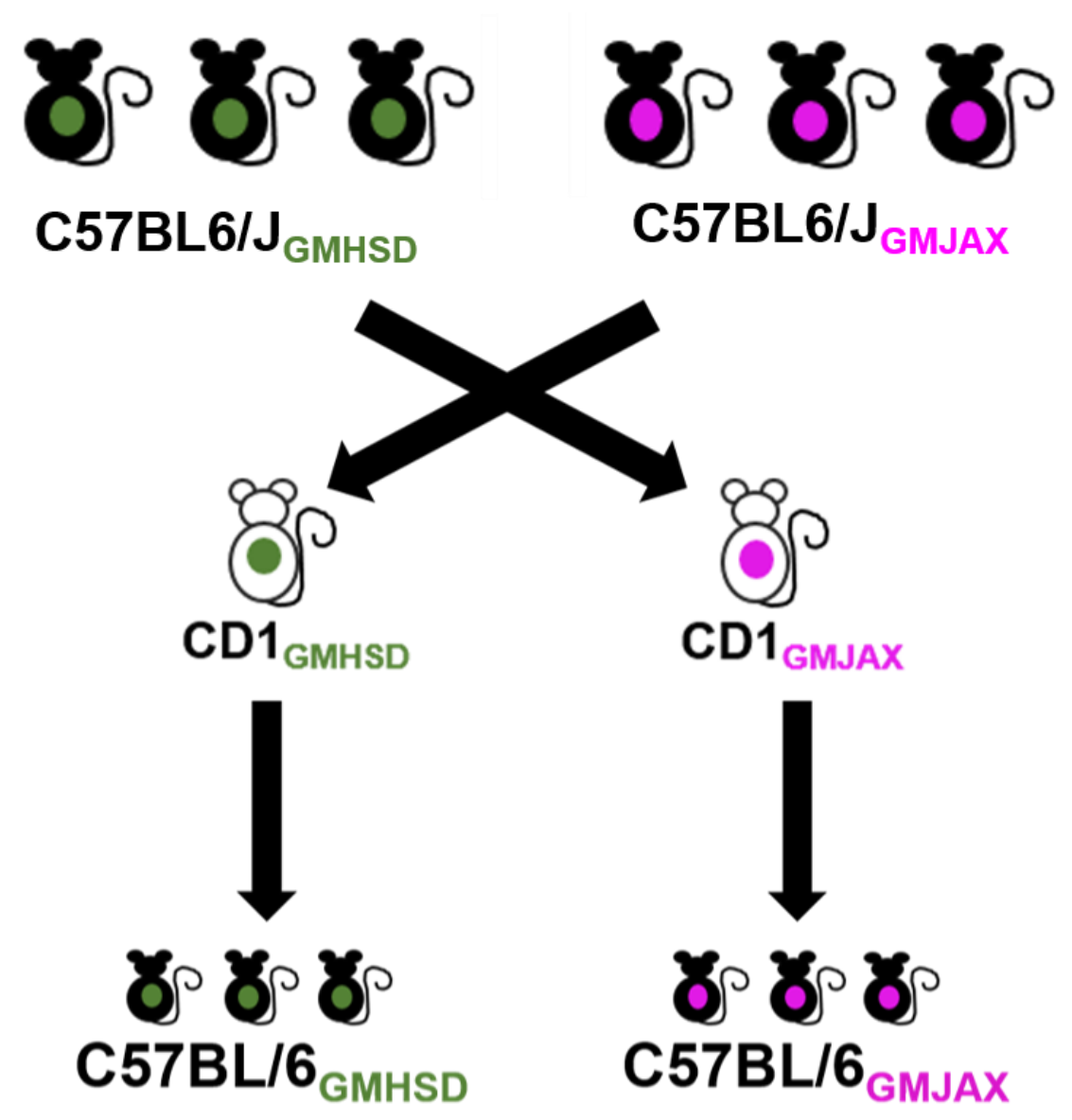


Figure 2. Analysis of $\alpha$-diversity measures of CF pup recipients and surrogate dam donors. Differences in GM richness (OTUs observed) (A) and $\alpha$-diversity (Shannon Index) (B) at 3 months of age are shown with scatter plots. Pups are classified based on their donor (e.g. Pups classified as GMJAX originated with GMHSD and were fostered onto GMJAX surrogate donors). The richness of the CF pups matched their surrogate donors in both GMJAX and GMHSD pups. There was one exception in which a GMJAX pup maintained the high richness associated with its original GMHSD colonization. In both GMJAX and GMHSD, pups seem to display higher $\alpha$-diversity than their surrogate donors, but statistical comparisons between the donor dams and their fostered pups cannot be performed due to the low number of surrogates. CF pups with a GMHSD donor has significantly higher richness and $\alpha$-diversity than CF pups with GMJAX donors. $* * * p<0.001$; CF pups were statistically compared using a Student's t-test (dams could not be included due to low experimental numbers).

\section{A}

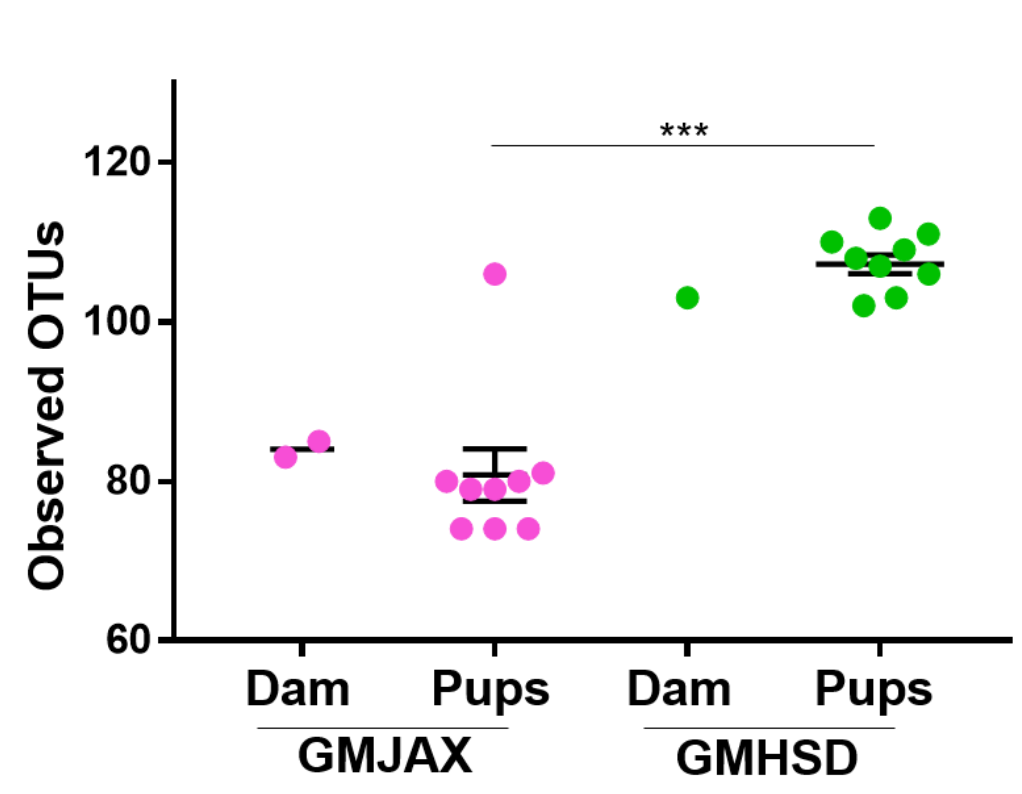


B

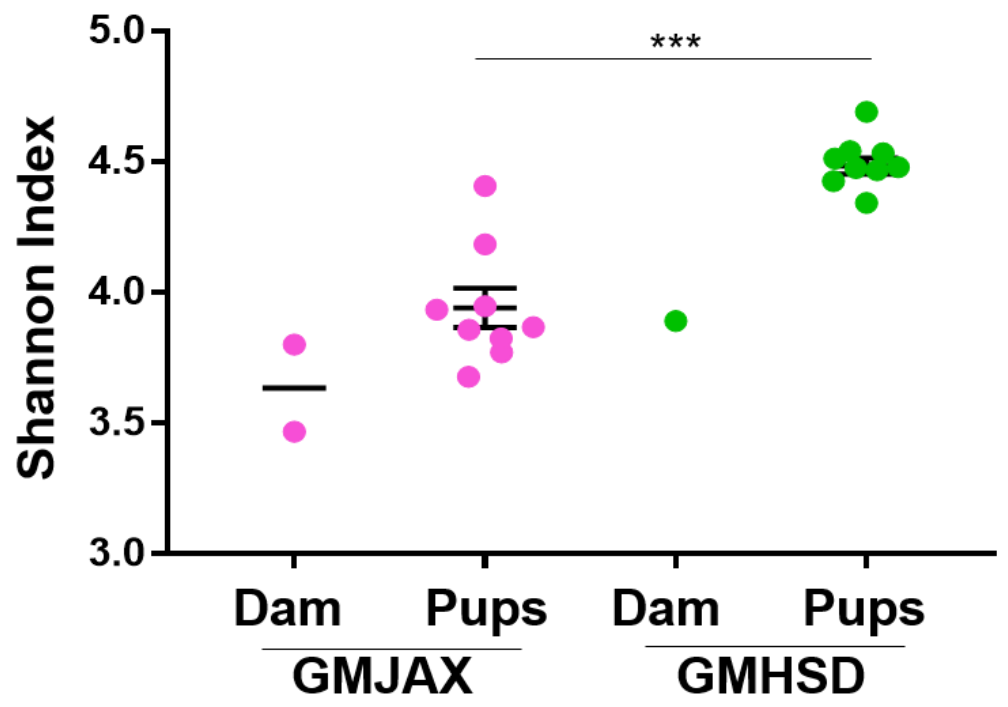


Figure 3. Analysis of $\beta$-diversity measures of CF pup recipients and surrogate dam donors. Unweighted PCoA representing differences in $\beta$-diversity at the Operational Taxanomic Unit (OTU) level between GMJAX and GMHSD CF pups and their surrogate GM donors at weaning (A) and 3 months of age (B). CF pups clustered with their surrogate donors along principal coordinate 1 at weaning and 3 months of age, regardless of their original GM colonization. At weaning, there was separation noted between surrogate donors and their CF pups along principal coordinate 2. There was also one GMJAX pup outlier at weaning, which appears to have a hybrid of GMJAX and GMHSD.

\section{A}

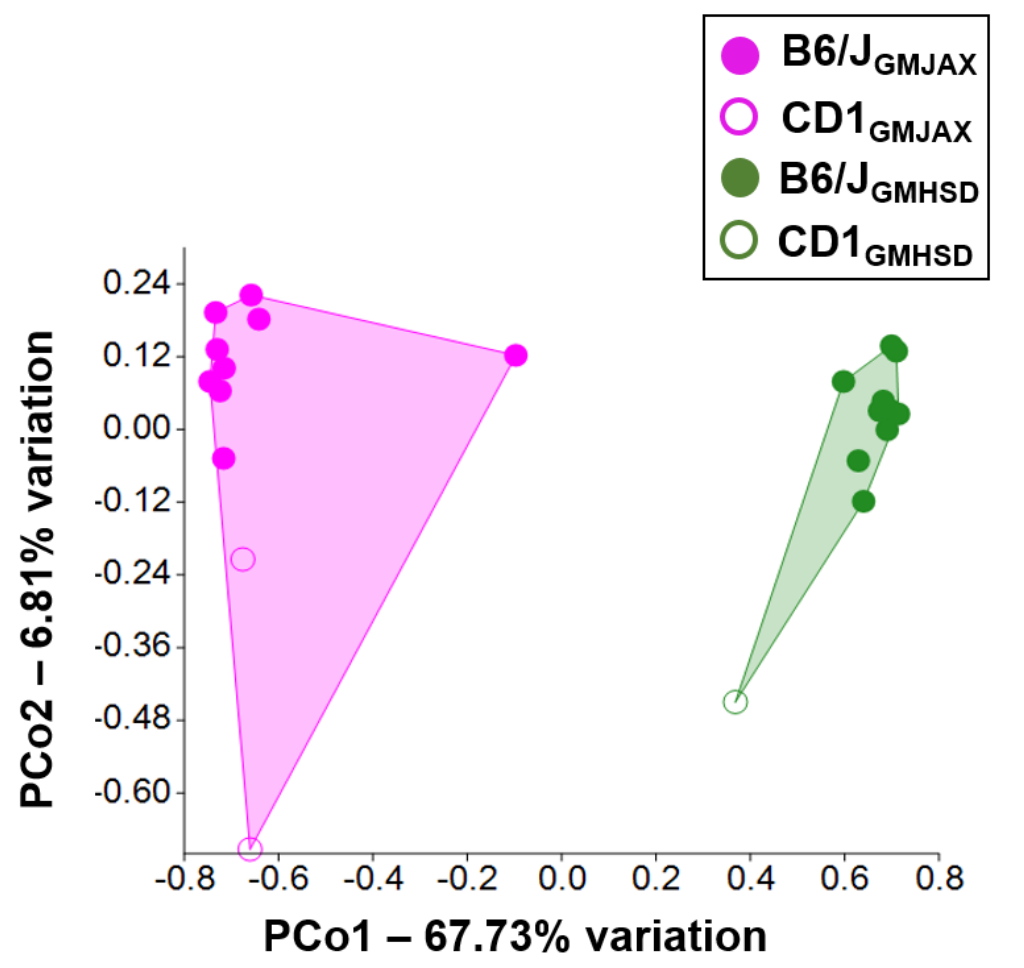


B

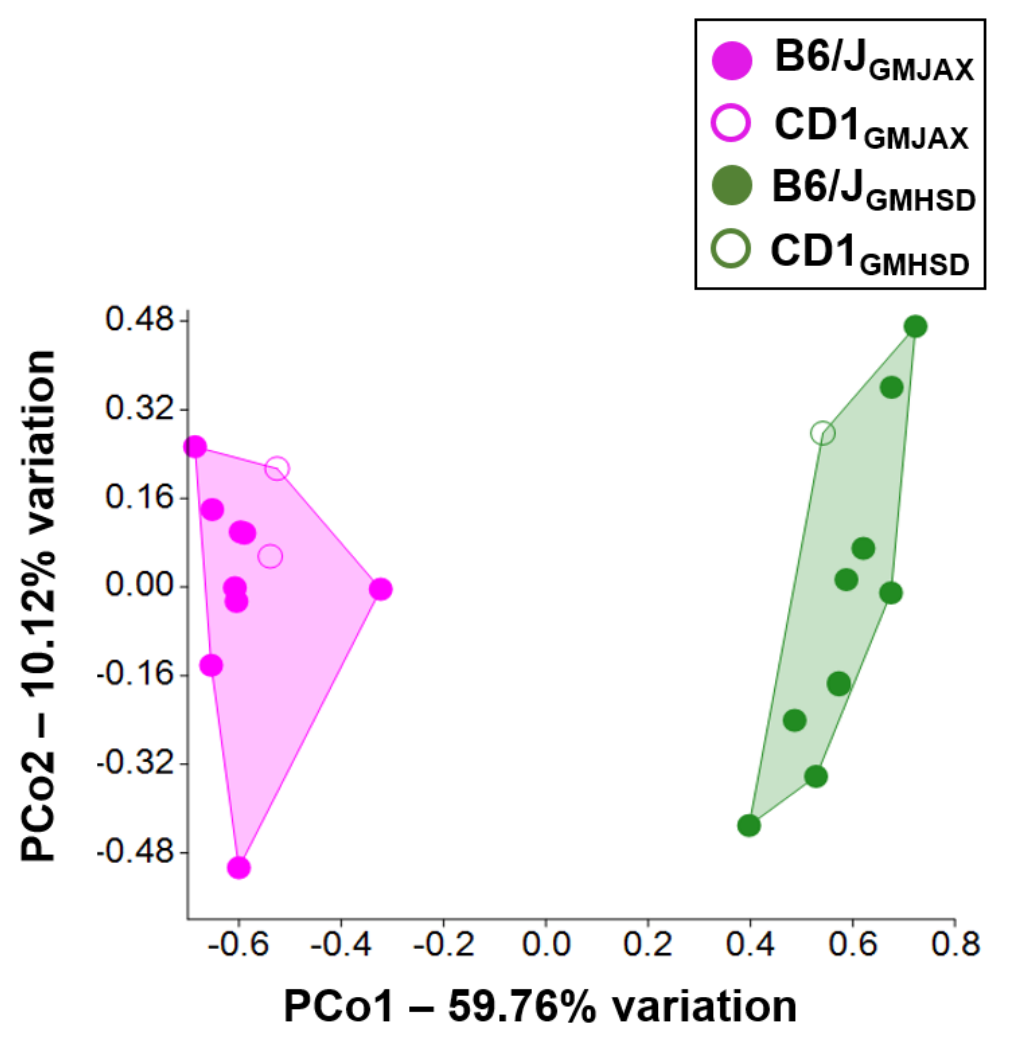




\section{B.4. Discussion}

In this preliminary study, we tested whether cross fostering can be used to transfer the complex GM from surrogate donor to recipient. We specifically designed this experiment to assess whether the recipient and donor GM richness and diversity influence overall effectiveness of CF. To address this, we used B6/J recipients colonized with either a lower richness/diversity GM (GMJAX) or higher richness/diversity (GMHSD), and cross fostered them onto timed-mated surrogates harboring the opposite GM within 12 hours of birth. To follow, we evaluated richness, $\alpha$-diversity, and $\beta$-diversity measures at weaning and 3 months of age to determine whether GM transfer was successful.

We found that regardless of whether the recipient began with GMJAX or GMHSD, the richness (OTUs observed) of the surrogate donors was transferred to the CF recipients. This indicates that the OTUs present in the surrogate donors were largely transferred to the recipients, though further analysis is required to confirm that the same OTUs are present. Interestingly, one significant outlier was observed in the B6/J pups transferred to GMJAX surrogates (Fig. 2A). This outlier displayed a higher richness, which was similar to the richness of the GMHSD surrogates and their recipients. This suggests that in this particular individual, there was not successful transfer from the highrichness GMHSD to the low-richness GMJAX. In contrast, both GMJAX and GMHSD B6/J recipients demonstrated a higher $\alpha$-diversity (Shannon index) than their surrogate counterparts (Fig. 2B). Presently, the cause of this phenomenon is unclear, and larger surrogate donor sample sizes are required to statistically compare the surrogate dams and recipient pups to determine whether this observation persists. Taken together, these 
results indicate successful transfer of OTUs from donor to recipient via CF, but suggest that $\mathrm{CF}$ affects the distribution of these OTUs as determined by $\alpha$-diversity.

We also performed $\beta$-diversity community analysis by Principal Coordinate Analysis (PCoA) using fecal samples from weaning and 3-month old B6/J recipients and their surrogate donors. At weaning, we noted clustering of B6/J recipients and their surrogate donors along PCo1, indicating overall community similarity between the two. However, we observed separation between the surrogate donors and their CF pups on $\mathrm{PCo} 2$ at weaning. Interestingly, at 3 months of age, this separation was no longer apparent as the surrogate donors clustered with their CF recipients along both PCo1 and PCo2, suggesting that age of sampling was an important factor. Of note, the aforementioned GMJAX outlier in Fig. 2A, which maintained a high GM richness, was also an outlier in our $\beta$-diversity community analysis. At weaning, this individual's fecal sample was plotted between the GMJAX and GMHSD clusters, indicating that it was likely a hybrid of the two GMs.

To summarize, we achieved relatively successful GM transfer via cross fostering, regardless of whether the recipients started with a low or high richness/diversity. However, there were substantial caveats and limitations to our conclusions from this preliminary study. Notably, $\alpha$-diversity was consistently higher in recipients compared to their surrogate donors. Moreover, we observed a $\mathrm{B} 6 / \mathrm{J}_{\mathrm{GMHSD}}$ recipient that failed to transfer to the surrogate GMJAX microbiota. Thus, this study should be repeated with larger sample sizes to determine whether this failure of transfer is a true outlier. Overall, this work defines our early understanding of the effectiveness of various microbiota transfer techniques that are applicable to a wide range of research. 


\section{Bibliography}

Ajouz, H., D. Mukherji and A. Shamseddine (2014). "Secondary bile acids: an underrecognized cause of colon cancer." World Journal of Surgical Oncology 12(164).

Al-Tassan, N. A., N. Whiffin, F. J. Hosking, C. Palles, S. M. Farrington, S. E. Dobbins, R. Harris, M. Gorman, A. Tenesa, B. F. Meyer, S. M. Wakil, B. Kinnersley, H.

Campbell, L. Martin, C. G. Smith, S. Idziaszczyk, E. Barclay, T. S. Maughan, R. Kaplan, R. Kerr, D. Kerr, D. D. Buchanan, A. K. Win, J. Hopper, M. Jenkins, N. M. Lindor, P. A. Newcomb, S. Gallinger, D. Conti, F. Schumacher, G. Casey, M. G. Dunlop, I. P. Tomlinson, J. P. Cheadle and R. S. Houlston (2015). "A new GWAS and meta-analysis with 1000Genomes imputation identifies novel risk variants for colorectal cancer." Sci Rep 5: 10442 .

Alam, A., G. Leoni, M. Quiros, H. Wu, C. Desai, H. Nishio, R. M. Jones, A. Nusrat and A. S. Neish (2016). "The microenvironment of injured murine gut elicits a local prorestitutive microbiota." Nat Microbiol 1: 15021.

Alrawi, S. J., M. Schiff, R. E. Carroll, M. Dayton, J. F. Gibbs, M. Kulavlat, D. Tan, K. Berman, D. L. Stoler and G. R. Anderson (2006). "Aberrant Crypt Foci." Anticancer Research 26: 107-120.

Altschul, S. F., T. L. Madden, A. A. Schaffer, J. Zhang, Z. Zhang, W. Miller and D. J. Lipman (1997). "Gapped BLAST and PSI-BLAST: a new generation of protein database search programs." Nucleic Acids Research 25(17): 3389-3402.

Altschul, S. F., T. L. Madden, A. A. Schaffer, J. Zhang, Z. Zhang, W. Miller and D. J. Lipman (1997). "Gapped BLAST and PSI-BLAST: a new generation of protein database search programs." Nucleic Acids Res 25(17): 3389-3402.

Amitay, E. L., S. Werner, M. Vital, D. H. Pieper, D. Hofler, I. J. Gierse, J. Butt, Y. Balavarca, K. Cuk and H. Brenner (2017). "Fusobacterium and colorectal cancer: causal factor or passenger? Results from a large colorectal cancer screening study." Carcinogenesis 38(8): 781-788.

Amos-Landgraf, J. M., J. Heijmans, M. C. Wielenga, E. Dunkin, K. J. Krentz, L. Clipson, A. G. Ederveen, P. G. Groothuis, S. Mosselman, V. Muncan, D. W. Hommes, A. Shedlovsky, W. F. Dove and G. R. van den Brink (2014). "Sex disparity in colonic adenomagenesis involves promotion by male hormones, not protection by female hormones." Proc Natl Acad Sci U S A 111(46): 16514-16519.

Amos-Landgraf JM, H. J., Wielenga MCB, Dunkin E, Krentz KJ, et al. (2014). "Sex disparity in colonic adenomagenesis involves promotion by male hormones, not protection by female hormones." PNAS 111(46): 16514-16519. 
Amos-Landgraf, J. M., A. A. Irving, C. Hartman, A. Hunter, B. Laube, X. Chen, L. Clipson, M. A. Newton and W. F. Dove (2012). "Monoallelic silencing and haploinsufficiency in early murine intestinal neoplasms." Proceedings of the National Academy of Sciences 109(6): 2060-2065.

Amos-Landgraf, J. M., L. N. Kwong, C. M. Kendziorski, M. Reichelderfer, J. Torrealba, J. Weichert, J. D. Haag, K. Chen, J. L. Waller, M. N. Gould and W. F. Dove (2007). "A target-selected $A p c$-mutant rat kindred enhances the modeling of familial human colon cancer." Proc Natl Acad Sci U S A 104(10): 4036-4041.

Aoki, K., Y. Tamai, S. Horiike, M. Oshima and M. M. Taketo (2003). "Colonic polyposis caused by mTOR-mediated chromosomal instability in Apc+/Delta716 Cdx2+/compound mutant mice." Nat Genet 35(4): 323-330.

Arthur, J. C. and C. Jobin (2011). "The struggle within: microbial influences on colorectal cancer." Inflamm Bowel Dis 17(1): 396-409.

Attene-Ramos, M. S., E. D. Wagner, H. R. Gaskins and M. J. Plewa (2007). "Hydrogen sulfide induces direct radical-associated DNA damage." Mol Cancer Res 5(5): 455-459.

Attene-Ramos, M. S., E. D. Wagner, M. J. Plewa and H. R. Gaskins (2006). "Evidence that hydrogen sulfide is a genotoxic agent." Mol Cancer Res 4(1): 9-14.

Ausubel FM, B. R., Kingston RE, Moore DD, Seidman JG, Smith JA, Struhl K (1994). Current Protocols in Molecular Biology. Current Protocols in Molecular Biology. New York, NY, John Wiley \& Sons. 2.

Backhed, F., H. Ding, T. Wang, L. V. Hooper, G. Y. Koh, A. Nagy, C. F. Semenkovich and J. I. Gordon (2004). "The gut microbiota as an environmental factor that regulates fat storage." Proc Natl Acad Sci U S A 101(44): 15718-15723.

Balamurugan, R., E. Rajendiran, S. George, G. V. Samuel and B. S. Ramakrishna (2008). "Real-time polymerase chain reaction quantification of specific butyrate-producing bacteria, Desulfovibrio and Enterococcus faecalis in the feces of patients with colorectal cancer." J Gastroenterol Hepatol 23(8 Pt 1): 1298-1303.

Bamola, V. D., A. Ghosh, R. K. Kapardar, B. Lal, S. Cheema, P. Sarma and R. Chaudhry (2017). "Gut microbial diversity in health and disease: experience of healthy Indian subjects, and colon carcinoma and inflammatory bowel disease patients." Microb Ecol Health Dis 28(1): 1322447.

Baran, A. A., K. A. Silverman, J. Zeskand, R. Koratkar, A. Palmer, K. McCullen, W. J. Curran, Jr., T. B. Edmonston, L. D. Siracusa and A. M. Buchberg (2007). "The modifier of Min 2 (Mom2) locus: embryonic lethality of a mutation in the Atp5a1 gene suggests a novel mechanism of polyp suppression." Genome Res 17(5): 566-576. 
Barcenilla, A., S. E. Pryde, J. C. Martin, S. H. Duncan, C. S. Stewart, C. Henderson and H. J. Flint (2000). "Phylogenetic Relationships of Butyrate-Producting Bacteria from the Human Gut." Applied and Environmental Microbiology 66(4): 1654-1661.

Baron, E. J., P. Summanen, J. Downes, M. C. Roberts, H. Wexler and S. M. Finegold (1989). "Bilophila wadsworthia, gen. nov. and sp. nov., a Unique Gram-negative Anaerobic Rod Recovered from Appendicitis Specimens and Human Faeces." Journal of General Microbiology 135: 3405-3411.

Baron, J. A., B. F. Cole, R. S. Sandler, R. W. Haile, D. Ahnen, R. Bresalier, G. McKeown-Eyssen, R. W. Summers, R. Rothstein, C. A. Burke, D. C. Snover, T. R. Church, J. I. Allen, M. Beach, G. J. Beck, J. H. Bond, T. Byers, R. Greenberg, J. S. Mandel, N. Marcon, L. A. Mott, L. Pearson, F. Saibil and R. U. van Stolk (2003). "A Randomized Trial of Aspirin to Prevent Colorectal Adenomas." The New England Journal of Medicine 348(10): 891-899.

Bertagnolli, M. M., M. Hsu, E. T. Hawk, C. J. Eagle, A. G. Zauber and I. Adenoma Prevention with Celecoxib Study (2010). "Statin use and colorectal adenoma risk: results from the adenoma prevention with celecoxib trial." Cancer Prev Res (Phila) 3(5): 588596.

Bidot, W. A., A. C. Ericsson and C. L. Franklin (2018). "Effects of water decontamination methods and bedding material on the gut microbiota." PLoS One 13(10): e0198305.

Bienz, M. and H. Clevers (2000). "Linking Colorectal Cancer to Wnt Signaling." Cell 103: $311-320$.

Biggers JD, M. L., Raffin M. (2000). "Amino Acids and Preimplantation Development of the Mouse in Protein-Free Potassium Simplex Optimized Medium." Biology of Reproduction 63: 281-293.

Bino, R. J., R. D. Hall, O. Fiehn, J. Kopka, K. Saito, J. Draper, B. J. Nikolau, P. Mendes, U. Roessner-Tunali, M. H. Beale, R. N. Trethewey, B. M. Lange, E. S. Wurtele and L. W. Sumner (2004). "Potential of metabolomics as a functional genomics tool." Trends Plant Sci 9(9): 418-425.

Birt, D. F. and G. J. Phillips (2014). "Diet, genes, and microbes: complexities of colon cancer prevention." Toxicol Pathol 42(1): 182-188.

Bordonaro, M. and D. L. Lazarova (2015). "CREB-binding protein, p300, butyrate, and Wnt signaling in colorectal cancer." World J Gastroenterol 21(27): 8238-8248.

Breuer, K., A. K. Foroushani, M. R. Laird, C. Chen, A. Sribnaia, R. Lo, G. L. Winsor, R. E. Hancock, F. S. Brinkman and D. J. Lynn (2013). "InnateDB: systems biology of innate 
immunity and beyond--recent updates and continuing curation." Nucleic Acids Res 41(Database issue): D1228-1233.

Brim, H., S. Yooseph, E. G. Zoetendal, E. Lee, M. Torralbo, A. O. Laiyemo, B. Shokrani, K. Nelson and H. Ashktorab (2013). "Microbiome analysis of stool samples from African Americans with colon polyps." PLoS One 8(12): e81352.

Brown, D. G., S. Rao, T. L. Weir, J. O'Malia, M. Bazan, R. J. Brown and E. P. Ryan (2016). "Metabolomics and metabolic pathway networks from human colorectal cancers, adjacent mucosa, and stool." Cancer Metab 4: 11.

Bryant, C. D. (2011). "The blessings and curses of C57BL/6 substrains in mouse genetic studies." Ann N Y Acad Sci 1245: 31-33.

Bultman, S. J. and C. Jobin (2014). "Microbial-derived butyrate: an oncometabolite or tumor-suppressive metabolite?" Cell Host Microbe 16(2): 143-145.

Cani, P. D. and W. M. de Vos (2017). "Next-Generation Beneficial Microbes: The Case of Akkermansia muciniphila." Front Microbiol 8: 1765.

Cao, H., S. Luo, M. Xu, Y. Zhang, S. Song, S. Wang, X. Kong, N. He, X. Cao, F. Yan and B. Wang (2014). "The secondary bile acid, deoxycholate accelerates intestinal adenoma-adenocarcinoma sequence in Apc ( $\mathrm{min} /+)$ mice through enhancing Wnt signaling." Fam Cancer 13(4): 563-571.

Caporaso, J. G., C. L. Lauber, W. A. Walters, D. Berg-Lyons, C. A. Lozupone, P. J. Turnbaugh, N. Fierer and R. Knight (2010). "Global patterns of 16S rRNA diversity at a depth of millions of sequences per sample." Proc Natl Acad Sci U S A 108: 4516-4522.

Carbonero, F., A. C. Benefiel, A. H. Alizadeh-Ghamsari and H. R. Gaskins (2012). "Microbial pathways in colonic sulfur metabolism and links with health and disease." Front Physiol 3: 448.

Carvalho, M. I., I. Pires, J. Prada and F. L. Queiroga (2014). "A role for T-lymphocytes in human breast cancer and in canine mammary tumors." Biomed Res Int 2014: 130894

Chae, W. J., T. F. Gibson, D. Zelterman, L. Hao, O. Henegariu and A. L. Bothwell (2010). "Ablation of IL-17A abrogates progression of spontaneous intestinal tumorigenesis." Proc Natl Acad Sci U S A 107(12): 5540-5544.

Chattopadhyay, M., R. Kodela, K. R. Olson and K. Kashfi (2012). "NOSH-aspirin (NBS1120), a novel nitric oxide- and hydrogen sulfide-releasing hybrid is a potent inhibitor of colon cancer cell growth in vitro and in a xenograft mouse model." Biochem Biophys Res Commun 419(3): 523-528. 
Chen, J. and L. Vitetta (2018). "Inflammation-Modulating Effect of Butyrate in the Prevention of Colon Cancer by Dietary Fiber." Clin Colorectal Cancer 17(3): e541-e544.

Chen, W., F. Liu, Z. Ling, X. Tong and C. Xiang (2012). "Human intestinal lumen and mucosa-associated microbiota in patients with colorectal cancer." PLoS One 7(6): e39743.

Cheng, K. and J. P. Raufman (2005). "Bile acid-induced proliferation of a human colon cancer cell line is mediated by transactivation of epidermal growth factor receptors." Biochem Pharmacol 70(7): 1035-1047.

Chiang, J. Y. (2009). "Bile acids: regulation of synthesis." J Lipid Res 50(10): 19551966.

Coenye, T. and P. Vandamme (2003). "Intragenomic heterogeneity between multiple 16S ribosomal RNA operons in sequenced bacterial genomes." FEMS Microbiology Letters 228(1): 45-49.

Collado, M. C., M. Derrien, E. Isolauri, W. M. de Vos and S. Salminen (2007). "Intestinal integrity and Akkermansia muciniphila, a mucin-degrading member of the intestinal microbiota present in infants, adults, and the elderly." Appl Environ Microbiol 73(23): 7767-7770.

Colnot, S., M. Niwa-Kawakita, G. Hamard, C. Godard, S. Le Plenier, C. Houbron, B. Romagnolo, D. Berrebi, M. Giovannini and C. Perret (2004). "Colorectal cancers in a new mouse model of familial adenomatous polyposis: influence of genetic and environmental modifiers." Lab Invest 84(12): 1619-1630.

Cormier, R. T., A. Bilger, A. J. Lillich, R. B. Halberg, K. H. Hong, K. A. Gould, N. Borenstein, E. S. Lander and W. F. Dove (2000). "The Mom $1^{\mathrm{AKR}}$ intestinal tumor resistance region consists of Pla2g2a and a locus distal to D4Mit64." Oncogene 19: 3182-3192.

Cortes-Ciriano, I., S. Lee, W.-Y. Park, T.-M. Kim and P. J. Park (2017). "A molecular portrait of microsatellite instability across multiple cancers." Nature Communications 8(1).

Costarelli, V., T. J. Key, P. N. Appleby, D. S. Allen, I. S. Fentiman and T. A. Sanders (2002). "A prospective study of serum bile acid concentrations and colorectal cancer risk in post-menopausal women on the island of Guernsey." Br J Cancer 86(11): 1741-1744.

Couturier-Maillard, A., T. Secher, A. Rehman, S. Normand, A. De Arcangelis, R. Haesler, L. Huot, T. Grandjean, A. Bressenot, A. Delanoye-Crespin, O. Gaillot, S. Schreiber, Y. Lemoine, B. Ryffel, D. Hot, G. Nunez, G. Chen, P. Rosenstiel and M. Chamaillard (2013). "NOD2-mediated dysbiosis predisposes mice to transmissible colitis and colorectal cancer." J Clin Invest 123(2): 700-711. 
Crist, R. C., J. J. Roth, A. A. Baran, B. J. McEntee, L. D. Siracusa and A. M. Buchberg (2010). "The armadillo repeat domain of Apc suppresses intestinal tumorigenesis." Mamm Genome 21(9-10): 450-457.

Cuevas-Ramos, G., C. R. Petit, I. Marcq, M. Boury, E. Oswald and J. P. Nougayrede (2010). "Escherichia coli induces DNA damage in vivo and triggers genomic instability in mammalian cells." Proc Natl Acad Sci U S A 107(25): 11537-11542.

Cunningham, J. M., E. R. Christensen, D. J. Tester, C. Kim, P. C. Roche, L. J. Burgart and S. N. Thibodeau (1998). "Hypermethylation of the $h M L H 1$ Promoter in Colon Cancer with Microsatellite Instability." Cancer Res 58: 3455-3460.

Danaei, G., S. Vander Hoorn, A. D. Lopez, C. J. L. Murray and M. Ezzati (2005). "Causes of cancer in the world: comparative risk assessment of nine behavioural and environmental risk factors." The Lancet 366(9499): 1784-1793.

Danecek, P., A. Auton, G. Abecasis, C. A. Albers, E. Banks, M. A. DePristo, R. E. Handsaker, G. Lunter, G. T. Marth, S. T. Sherry, G. McVean, R. Durbin and G. Genomes Project Analysis (2011). "The variant call format and VCFtools." Bioinformatics 27(15): 2156-2158.

Daniela, E., S. Sara, M. Marcella, A. Giovanni, A. Meynier, V. Sophie and C. M. Cristina (2014). "Fecal water genotoxicity in healthy free-living young Italian people." Food Chem Toxicol 64: 104-109.

de la Chapelle, A. (2004). "Genetic predisposition to colorectal cancer." Nat Rev Cancer 4(10): 769-780.

De Simone, V., F. Pallone, G. Monteleone and C. Stolfi (2013). "Role of TH17 cytokines in the control of colorectal cancer." Oncoimmunology 2(12): e26617.

Degirolamo, C., S. Modica, G. Palasciano and A. Moschetta (2011). "Bile acids and colon cancer: Solving the puzzle with nuclear receptors." Trends Mol Med 17(10): 564572.

DeHaven, C. D., A. M. Evans, H. Dai and K. A. Lawton (2010). "Organization of GC/MS and LC/MS metabolomics data into chemical libraries." Journal of Cheminformatics 2(9).

Dejea, C. M., E. C. Wick, E. M. Hechenbleikner, J. R. White, J. L. Mark Welch, B. J. Rossetti, S. N. Peterson, E. C. Snesrud, G. G. Borisy, M. Lazarev, E. Stein, J. Vadivelu, A. C. Roslani, A. A. Malik, J. W. Wanyiri, K. L. Goh, I. Thevambiga, K. Fu, F. Wan, N. Llosa, F. Housseau, K. Romans, X. Wu, F. M. McAllister, S. Wu, B. Vogelstein, K. W. Kinzler, D. M. Pardoll and C. L. Sears (2014). "Microbiota organization is a distinct 
feature of proximal colorectal cancers." Proc Natl Acad Sci U S A 111(51): 1832118326.

Dermadi, D., S. Valo, S. Ollila, R. Soliymani, N. Sipari, M. Pussila, L. Sarantaus, J. Linden, M. Baumann and M. Nystrom (2017). "Western Diet Deregulates Bile Acid Homeostasis, Cell Proliferation, and Tumorigenesis in Colon." Cancer Res 77(12): 33523363.

Derrien, M., M. C. Collado, K. Ben-Amor, S. Salminen and W. M. de Vos (2008). "The Mucin degrader Akkermansia muciniphila is an abundant resident of the human intestinal tract." Appl Environ Microbiol 74(5): 1646-1648.

Derrien, M., P. Van Baarlen, G. Hooiveld, E. Norin, M. Muller and W. M. de Vos (2011). "Modulation of Mucosal Immune Response, Tolerance, and Proliferation in Mice Colonized by the Mucin-Degrader Akkermansia muciniphila." Front Microbiol 2: 166.

Desai, M. S., A. M. Seekatz, N. M. Koropatkin, N. Kamada, C. A. Hickey, M. Wolter, N. A. Pudlo, S. Kitamoto, N. Terrapon, A. Muller, V. B. Young, B. Henrissat, P. Wilmes, T. S. Stappenbeck, G. Nunez and E. C. Martens (2016). "A Dietary Fiber-Deprived Gut Microbiota Degrades the Colonic Mucus Barrier and Enhances Pathogen Susceptibility." Cell 167(5): 1339-1353 e1321.

Devkota, S., Y. Wang, M. W. Musch, V. Leone, H. Fehlner-Peach, A. Nadimpalli, D. A. Antonopoulos, B. Jabri and E. B. Chang (2012). "Dietary-fat-induced taurocholic acid promotes pathobiont expansion and colitis in Il10-/- mice." Nature 487(7405): 104-108.

Dietrich, W. F., E. S. Lander, J. S. Smith, A. R. Moser, K. A. Gould, C. Luongo, N. Borenstein and W. F. Dove (1993). "Genetic Identification of Mom-1, a Major Modifier Locus Affecting Min-Induced Intestinal Neoplasia in the Mouse." Cell 75: 631-639.

Dingemanse, C., C. Belzer, S. A. van Hijum, M. Gunthel, D. Salvatori, J. T. den Dunnen, E. J. Kuijper, P. Devilee, W. M. de Vos, G. B. van Ommen and E. C. Robanus-Maandag (2015). "Akkermansia muciniphila and Helicobacter typhlonius modulate intestinal tumor development in mice." Carcinogenesis 36(11): 1388-1396.

Donohoe, D. R., D. Holley, L. B. Collins, S. A. Montgomery, A. C. Whitmore, A. Hillhouse, K. P. Curry, S. W. Renner, A. Greenwalt, E. P. Ryan, V. Godfrey, M. T. Heise, D. S. Threadgill, A. Han, J. A. Swenberg, D. W. Threadgill and S. J. Bultman (2014). "A gnotobiotic mouse model demonstrates that dietary fiber protects against colorectal tumorigenesis in a microbiota- and butyrate-dependent manner." Cancer Discov 4(12): 1387-1397.

Doran, A. G., K. Wong, J. Flint, D. J. Adams, K. W. Hunter and T. M. Keane (2016). "Deep genome sequencing and variation analysis of 13 inbred mouse strains defines candidate phenotypic alleles, private variation and homozygous truncating mutations." Genome Biol 17(1): 167. 
Dorman, A., D. Baer, I. Tomlinson, R. Mott and F. A. Iraqi (2016). "Genetic analysis of intestinal polyp development in Collaborative Cross mice carrying the Apc (Min/+) mutation." BMC Genet 17: 46.

Dossa, A. Y., O. Escobar, J. Golden, M. R. Frey, H. R. Ford and C. P. Gayer (2016). "Bile acids regulate intestinal cell proliferation by modulating EGFR and FXR signaling." Am J Physiol Gastrointest Liver Physiol 310(2): G81-92.

Ericsson, A. C., S. Akter, M. M. Hanson, S. B. Busi, T. W. Parker, R. J. Schehr, M. A. Hankins, C. E. Ahner, J. W. Davis, C. L. Franklin, J. M. Amos-Landgraf and E. C. Bryda (2015). "Differential susceptibility to colorectal cancer due to naturally occurring gut microbiota." Oncotarget 6(32): 33689-33704.

Ericsson, A. C., J. W. Davis, W. Spollen, N. Bivens, S. Givan, C. E. Hagan, M. McIntosh and C. L. Franklin (2015). "Effects of vendor and genetic background on the composition of the fecal microbiota of inbred mice." PLoS One 10(2): e0116704.

Everard, A., C. Belzer, L. Geurts, J. P. Ouwerkerk, C. Druart, L. B. Bindels, Y. Guiot, M. Derrien, G. G. Muccioli, N. M. Delzenne, W. M. de Vos and P. D. Cani (2013). "Crosstalk between Akkermansia muciniphila and intestinal epithelium controls diet-induced obesity." Proc Natl Acad Sci U S A 110(22): 9066-9071.

Farhana, L., P. Nangia-Makker, E. Arbit, K. Shango, S. Sarkar, H. Mahmud, T. Hadden, Y. Yu and A. P. Majumdar (2016). "Bile acid: a potential inducer of colon cancer stem cells." Stem Cell Res Ther 7(1): 181.

Fearon, E. R. and B. Vogelstein (1990). "A Genetic Model for Colorectal Tumorigenesis." Cell 61: 759-767.

Fontaine, D. A. and D. B. Davis (2016). "Attention to Background Strain Is Essential for Metabolic Research: C57BL/6 and the International Knockout Mouse Consortium." Diabetes 65(1): 25-33.

Forster, G. M., K. Raina, A. Kumar, S. Kumar, R. Agarwal, M. H. Chen, J. E. Bauer, A. M. McClung and E. P. Ryan (2013). "Rice varietal differences in bioactive bran components for inhibition of colorectal cancer cell growth." Food Chem 141(2): 15451552.

Fotiadis, C. I., C. N. Stoidis, B. G. Spyropoulos and E. D. Zografos (2008). "Role of probiotics, prebiotics and synbiotics in chemoprevention for colorectal cancer." World J Gastroenterol 14(42): 6453-6457.

Franklin, C. L. and A. C. Ericsson (2017). "Microbiota and reproducibility of rodent models." Lab Anim (NY) 46(4): 114-122. 
Franzosa, E. A., A. Sirota-Madi, J. Avila-Pacheco, N. Fornelos, H. J. Haiser, S. Reinker, T. Vatanen, A. B. Hall, H. Mallick, L. J. McIver, J. S. Sauk, R. G. Wilson, B. W. Stevens, J. M. Scott, K. Pierce, A. A. Deik, K. Bullock, F. Imhann, J. A. Porter, A. Zhernakova, J. Fu, R. K. Weersma, C. Wijmenga, C. B. Clish, H. Vlamakis, C. Huttenhower and R. J. Xavier (2019). "Gut microbiome structure and metabolic activity in inflammatory bowel disease." Nat Microbiol 4(2): 293-305.

Frigola, J., J. Song, C. Stirzaker, R. A. Hinshelwood, M. A. Peinado and S. J. Clark (2006). "Epigenetic remodeling in colorectal cancer results in coordinate gene suppression across an entire chromosome band." Nat Genet 38(5): 540-549.

Fuchs, M. (2003). "Bile Acid Regulation of Hepatic Physiology III. Rregulation of bile acid synthesis: past progress and future challenges." Am J Physiol Gastrointest Liver Physiol 284: G551-G559.

Fukugaiti, M. H., A. Ignacio, M. R. Fernandes, U. Ribeiro Junior, V. Nakano and M. J. Avila-Campos (2015). "High occurrence of Fusobacterium nucleatum and Clostridium difficile in the intestinal microbiota of colorectal carcinoma patients." Braz J Microbiol 46(4): 1135-1140.

Goodwin, A. C., C. E. Destefano Shields, S. Wu, D. L. Huso, X. Wu, T. R. MurrayStewart, A. Hacker-Prietz, S. Rabizadeh, P. M. Woster, C. L. Sears and R. A. Casero, Jr. (2011). "Polyamine catabolism contributes to enterotoxigenic Bacteroides fragilisinduced colon tumorigenesis." Proc Natl Acad Sci U S A 108(37): 15354-15359.

Goss, K. H., M. A. Risinger, J. J. Kordich, M. M. Sanz, J. E. Straughen, L. E. Slovek, A. H. Capobianco, J. German, G. P. Boivin and J. Groden (2002). "Enhanced Tumor Formation in Mice Heterozygous for Blm Mutation." Science 297: 2051-2053.

Groden, J., A. Thliveris, W. Samowitz, M. Carlson, L. Gelbert, H. Albertsen, G. Joslyn, J. Stevens, L. Spirio, M. Robertson, L. Sargeant, K. Krapcho, E. Wolff, R. Burt, J. P. Hughes, J. Warrington, J. McPherson, J. Wasmuth, D. L. Paslier, H. Abderrahim, D. Cohen, M. Leppert and R. White (1991). "Identification and Characterization of the Familial Adenomatous Polyposis Coli Gene." Cell 66: 589-600.

Gu, Y., X. Wang, J. Li, Y. Zhang, H. Zhong, R. Liu, D. Zhang, Q. Feng, X. Xie, J. Hong, H. Ren, W. Liu, J. Ma, Q. Su, H. Zhang, J. Yang, X. Wang, X. Zhao, W. Gu, Y. Bi, Y. Peng, X. Xu, H. Xia, F. Li, X. Xu, H. Yang, G. Xu, L. Madsen, K. Kristiansen, G. Ning and W. Wang (2017). "Analyses of gut microbiota and plasma bile acids enable stratification of patients for antidiabetic treatment." Nat Commun 8(1): 1785.

Guo, F. F., T. C. Yu, J. Hong and J. Y. Fang (2016). "Emerging Roles of Hydrogen Sulfide in Inflammatory and Neoplastic Colonic Diseases." Front Physiol 7: 156.

Hammer, O., D. A. T. Harper and P. D. Ryan (2001). "PAST: Paleontological Statistics Software Package for Education and Data Analysis." Palaeontological Electronica 4(1). 
Hart, M. L., A. C. Ericsson and C. L. Franklin (2017). "Differing Complex Microbiota Alter Disease Severity of the IL-10(-/-) Mouse Model of Inflammatory Bowel Disease." Front Microbiol 8: 792.

Hart, M. L., A. C. Ericsson, K. C. K. Lloyd, K. N. Grimsrud, A. R. Rogala, V. L. Godfrey, J. N. Nielsen and C. L. Franklin (2018). "Development of outbred CD1 mouse colonies with distinct standardized gut microbiota profiles for use in complex microbiota targeted studies." Sci Rep 8(1): 10107.

Hebels, D. G., K. M. Sveje, M. C. de Kok, M. H. van Herwijnen, G. G. Kuhnle, L. G. Engels, C. B. Vleugels-Simon, W. G. Mares, M. Pierik, A. A. Masclee, J. C. Kleinjans and T. M. de Kok (2012). "Red meat intake-induced increases in fecal water genotoxicity correlate with pro-carcinogenic gene expression changes in the human colon." Food Chem Toxicol 50(2): 95-103.

Hellmich, M. R. and C. Szabo (2015). "Hydrogen Sulfide and Cancer." Handb Exp Pharmacol 230: 233-241.

Holmes, E., J. V. Li, T. Athanasiou, H. Ashrafian and J. K. Nicholson (2011). "Understanding the role of gut microbiome-host metabolic signal disruption in health and disease." Trends Microbiol 19(7): 349-359.

Houlston, R. S., J. Cheadle, S. E. Dobbins, A. Tenesa, A. M. Jones, K. Howarth, S. L. Spain, P. Broderick, E. Domingo, S. Farrington, J. G. Prendergast, A. M. Pittman, E. Theodoratou, C. G. Smith, B. Olver, A. Walther, R. A. Barnetson, M. Churchman, E. E. Jaeger, S. Penegar, E. Barclay, L. Martin, M. Gorman, R. Mager, E. Johnstone, R. Midgley, I. Niittymaki, S. Tuupanen, J. Colley, S. Idziaszczyk, C. Consortium, H. J. Thomas, A. M. Lucassen, D. G. Evans, E. R. Maher, C. Consortium, C. C. Group, C. C. Group, T. Maughan, A. Dimas, E. Dermitzakis, J. B. Cazier, L. A. Aaltonen, P. Pharoah, D. J. Kerr, L. G. Carvajal-Carmona, H. Campbell, M. G. Dunlop and I. P. Tomlinson (2010). "Meta-analysis of three genome-wide association studies identifies susceptibility loci for colorectal cancer at 1q41,3q26.2, 12q13.13 and 20q13.33." Nat Genet 42(11): 973-977.

Imray, C. H. E., S. Radley, A. Davis, G. Barker, C. W. Hendrickse, I. A. Donovan, A. M. Lawson, P. R. Baker and J. P. Neoptolemos (1992). "Faecal unconjugated bile acids in patients with colorectal cancer or polyps." Gut 33: 1239-1245.

Islam, K. B., S. Fukiya, M. Hagio, N. Fujii, S. Ishizuka, T. Ooka, Y. Ogura, T. Hayashi and A. Yokota (2011). "Bile acid is a host factor that regulates the composition of the cecal microbiota in rats." Gastroenterology 141(5): 1773-1781.

Itzkowitz, S. H. and X. Yio (2004). "Inflammatation and Cancer IV. Colorectal cancer in inflammatory bowel disease: the role of inflammation." Am J Physiol Gastrointest Liver Physiol 287: G7-G17. 
Jackstadt, R. and O. J. Sansom (2016). "Mouse models of intestinal cancer." J Pathol 238(2): 141-151.

Jacoby, R. F., K. Seibert, C. E. Cole, G. Kelloff and R. A. Lubet (2000). "The Cyclooxygenase-2 Inhibitor Celecoxib Is a Potent Preventative and Therapeutic Agent in the Min Mouse Model of Adenomatous Polyposis." Cancer Research 60: 5040-5044.

Jandhyala, S. M., R. Talukdar, C. Subramanyam, H. Vuyyuru, M. Sasikala and D. Nageshwar Reddy (2015). "Role of the normal gut microbiota." World J Gastroenterol 21(29): 8787-8803.

Joshi-Tope, G., M. Gillespie, I. Vastrik, P. D'Eustachio, E. Schmidt, B. de Bono, B. Jassal, G. R. Gopinath, G. R. Wu, L. Matthews, S. Lewis, E. Birney and L. Stein (2005). "Reactome: a knowledgebase of biological pathways." Nucleic Acids Res 33(Database issue): D428-432.

Kanehisa, M. and S. Goto (2000). "KEGG: Kyoto Encylcopedia of Genes and Genomes." Nucleic Acids Res 28(1): 27-30.

Kinzler, K. W. and B. vogelstein (1996). "Lessons from hereditary colorectal cancer." Cell 87: 159-170.

Knudson, A. G. (1993). "Antioncogenes and human cancer." Proc Natl Acad Sci U S A 90: 10914-10921.

Korpela, K., P. Costea, L. P. Coelho, S. Kandels-Lewis, G. Willemsen, D. I. Boomsma, N. Segata and P. Bork (2018). "Selective maternal seeding and environment shape the human gut microbiome." Genome Res 28(4): 561-568.

Kostic, A. D., E. Chun, L. Robertson, J. N. Glickman, C. A. Gallini, M. Michaud, T. E. Clancy, D. C. Chung, P. Lochhead, G. L. Hold, E. M. El-Omar, D. Brenner, C. S. Fuchs, M. Meyerson and W. S. Garrett (2013). "Fusobacterium nucleatum potentiates intestinal tumorigenesis and modulates the tumor-immune microenvironment." Cell Host Microbe 14(2): 207-215.

Kostic, A. D., D. Gevers, C. S. Pedamallu, M. Michaud, F. Duke, A. M. Earl, A. I. Ojesina, J. Jung, A. J. Bass, J. Tabernero, J. Baselga, C. Liu, R. A. Shivdasani, S. Ogino, B. W. Birren, C. Huttenhower, W. S. Garrett and M. Meyerson (2012). "Genomic analysis identifies association of Fusobacterium with colorectal carcinoma." Genome Res 22(2): 292-298.

Kuczynski, J., J. Stombaugh, W. A. Walters, A. Gonzalez, J. G. Caporaso and R. Knight (2012). "Using QIIME to analyze 16S rRNA gene sequences from microbial communities." Curr Protoc Microbiol Chapter 1: Unit 1E 5. 
Kwong, L. N. and W. F. Dove (2009). "APC and its modifiers in colon cancer." Adv Exp Med Biol 656: 85-106.

Kwong, L. N., A. Shedlovsky, B. S. Biehl, L. Clipson, C. A. Pasch and W. F. Dove (2007). "Identification of Mom7, a novel modifier of Apc(Min/+) on mouse chromosome 18." Genetics 176(2): 1237-1244.

Larsen, J. M. (2017). "The immune response to Prevotella bacteria in chronic inflammatory disease." Immunology 151(4): 363-374.

Laue, H., M. Friedrich, J. Ruff and A. M. Cook (2001). "Dissimilatory sulfite reductase (desulfoviridin) of the taurine-degrading, non-sulfate-reducing bacterium Bilophila wadsworthia RZATAU contains a fused DsrB-DsrD subunit." J Bacteriol 183(5): 17271733.

Lefebvre, P., B. Cariou, F. Lien, F. Kuipers and B. Staels (2009). "Role of bile acids and bile acid receptors in metabolic regulation." Physiol Rev 89(1): 147-191.

Li, H., B. Handsaker, A. Wysoker, T. Fennell, J. Ruan, N. Homer, G. Marth, G. Abecasis, R. Durbin and S. Genome Project Data Processing (2009). "The Sequence Alignment/Map format and SAMtools." Bioinformatics 25(16): 2078-2079.

Li, S., Y. Park, S. Duraisingham, F. H. Strobel, N. Khan, Q. A. Soltow, D. P. Jones and B. Pulendran (2013). "Predicting network activity from high throughput metabolomics." PLoS Comput Biol 9(7): e1003123.

Li, Y., P. Kundu, S. W. Seow, C. T. de Matos, L. Aronsson, K. C. Chin, K. Karre, S. Pettersson and G. Greicius (2012). "Gut microbiota accelerate tumor growth via c-jun and STAT3 phosphorylation in APCMin/+ mice." Carcinogenesis 33(6): 1231-1238.

Lichtenstein, P., N. V. Holm, P. K. Verkasalo, A. Iliadou, J. Kaprio, M. Koskenvuo, E. Pukkala, A. Skytthe and K. Hemminki (2000). "Environmental and heritable factors in the causation of cancer." The New England Journal of Medicine 343(2): 78-85.

Louis, P., G. L. Hold and H. J. Flint (2014). "The gut microbiota, bacterial metabolites and colorectal cancer." Nat Rev Microbiol 12(10): 661-672.

Lozupone, C. A., J. I. Stombaugh, J. I. Gordon, J. K. Jansson and R. Knight (2012). "Diversity, stability and resilience of the human gut microbiota." Nature 489(7415): 220230.

MacGregor, D. J., Y. S. Kim, M. H. Sleisenger and L. K. Johnson (2000). "Chemoprevention of colon cancer carcinogenesis by balsalazide: inhibition of azoxymethane-induced aberrant crypt formation in the rat colon and intestinal tumor formation in the B6-Min/+ mouse." International Journal of Oncology 17(1): 173-179. 
Mai, V., L. H. Colbert, D. Berrigan, S. N. Perkins, R. Pfeiffer, J. A. Lavigne, E. Lanza, D. C. Haines, A. Schatzkin and S. D. Hursting (2003). "Calorie Resitriction and Diet Composition Modulate Spontaneous Intestinal Tumorigenesis in $A p c^{\mathrm{Min}}$ Mice through Different Mechanisms." Cancer Research 63: 1752-1755.

Mariat, D., O. Firmesse, F. Levenez, V. Guimaraes, H. Sokol, J. Dore, G. Corthier and J. P. Furet (2009). "The Firmicutes/Bacteroidetes ratio of the human microbiota changes with age." BMC Microbiol 9: 123.

Marsh, V., D. J. Winton, G. T. Williams, N. Dubois, A. Trumpp, O. J. Sansom and A. R. Clarke (2008). "Epithelial Pten is dispensable for intestinal homeostasis but suppresses adenoma development and progression after Apc mutation." Nat Genet 40(12): 14361444.

McKenna, A., M. Hanna, E. Banks, A. Sivachenko, K. Cibulskis, A. Kernytsky, K. Garimella, D. Altshuler, S. Gabriel, M. Daly and M. A. DePristo (2010). "The Genome Analysis Toolkit: a MapReduce framework for analyzing next-generation DNA sequencing data." Genome Res 20(9): 1297-1303.

McLaren, W., B. Pritchard, D. Rios, Y. Chen, P. Flicek and F. Cunningham (2010). "Deriving the consequences of genomic variants with the Ensembl API and SNP Effect Predictor." Bioinformatics 26(16): 2069-2070.

Melnik, A. V., R. R. da Silva, E. R. Hyde, A. A. Aksenov, F. Vargas, A. Bouslimani, I. Protsyuk, A. K. Jarmusch, A. Tripathi, T. Alexandrov, R. Knight and P. C. Dorrestein (2017). "Coupling Targeted and Untargeted Mass Spectrometry for MetabolomeMicrobiome-Wide Association Studies of Human Fecal Samples." Anal Chem 89(14): 7549-7559.

Mendes, M. C. S., D. S. Paulino, S. R. Brambilla, J. A. Camargo, G. F. Persinoti and J. B. C. Carvalheira (2018). "Microbiota modification by probiotic supplementation reduces colitis associated colon cancer in mice." World J Gastroenterol 24(18): 1995-2008.

Millar, C. B., J. Guy, O. J. Sansom, J. Selfridge, E. MacDougall, B. Hendrich, P. D. Keightley, S. M. Bishop, A. R. Clarke and A. Bird (2002). "Enhanced CpG Mutability and Tumorigenesis in MBD4-Deficient Mice." Science 297: 403-405.

Milovic, V., I. C. Teller, D. Faust, W. F. Caspary and J. Stein (2002). "Effects of deoxycholate on human colon cancer cells: apoptosis of proliferation." European Journal of Clinical Investigation 32: 29-34.

Miyoshi, Y., H. Nagase, H. Ando, A. Horii, S. Ichii, S. Nakatsuru, T. Aoki, Y. Miki, T. Mori and Y. Nakamura (1992). "Somatic mutations of the APC gene in colorectal tumors: mutation cluster region in the APC gene." Human Molecular Genetics 1(4): 229-233. 
Montonye, D. R., A. C. Ericsson, S. B. Busi, C. Lutz, K. Wardwell and C. L. Franklin (2018). "Acclimation and Institutionalization of the Mouse Microbiota Following Transportation." Front Microbiol 9: 1085.

Morin, P., A. B. Sparks, V. Korinek, N. Barker, H. Clevers, B. vogelstein and K. W. Kinzler (1997). "Activation of B-catenin-Tcf Signaling in Colon Cancer by Mutations in B-Catenin or APC." Science 275: 1787-1790.

Moser, A. R., H. C. Pitot and W. F. Dove (1990). "A Dominant Mutation That Predisposes to Multiple Intestinal Neoplasia in the Mouse." Science 247: 322-324.

Natividad, J. M., B. Lamas, H. P. Pham, M. L. Michel, D. Rainteau, C. Bridonneau, G. da Costa, J. van Hylckama Vlieg, B. Sovran, C. Chamignon, J. Planchais, M. L. Richard, P. Langella, P. Veiga and H. Sokol (2018). "Bilophila wadsworthia aggravates high fat diet induced metabolic dysfunctions in mice." Nat Commun 9(1): 2802.

Nguyen, T. T., T. T. Ung, N. H. Kim and Y. D. Jung (2018). "Role of bile acids in colon carcinogenesis." World J Clin Cases 6(13): 577-588.

Nishisho, I., Y. Nakamura, Y. Miyoshi, Y. Miki, H. Ando, A. Horii, K. Koyama, J. Utsunomiya, S. Baba, P. Hedge, A. Markham, A. J. Krush, G. Petersen, S. R. Hamilton, M. Nilbert, D. B. Levy, T. M. Bryan, A. C. Preisinger, K. J. Smith, L. Su, K. W. Kinzler and B. Vogelstein (1991). "Mutations of Chromosome 5q21 Genes in FAP and Colorectal Cancer Patients." Science 253(5020): 665-669.

Ohigashi, S., K. Sudo, D. Kobayashi, O. Takahashi, T. Takahashi, T. Asahara, K. Nomoto and H. Onodera (2013). "Changes of the intestinal microbiota, short chain fatty acids, and fecal pH in patients with colorectal cancer." Dig Dis Sci 58(6): 1717-1726.

Ohmachi, T., H. Inoue, K. Mimori, F. Tanaka, A. Sasaki, T. Kanda, H. Fujii, K. Yanaga and M. Mori (2006). "Fatty acid binding protein 6 is overexpressed in colorectal cancer." Clin Cancer Res 12(17): 5090-5095.

Osawa, E., A. Nakajima, T. Fujisawa, Y. I. Kawamura, N. Toyama-Sorimachi, H. Nakagama and T. Dohi (2006). "Predominant T helper type 2-inflammatory responses promote murine colon cancers." Int J Cancer 118(9): 2232-2236.

Oshima, M., H. Oshima, K. Kitagawa, M. Kobayashi, C. Itakura and M. Taketo (1995). "Loss of $A p c$ heterozygosity and abnormal tissue building in nascent intestinal polyps in mice carrying a truncated Apc gene." Proc Natl Acad Sci U S A 92: 4482-4486.

Pages, F., A. Berger, M. Camus, F. Sanchez-Cabo, A. Costes, R. Molidor, B. Mlecnik, K. A., M. Nilsson, D. Damotte, T. Meatchi, P. Bruneval, P. Cuhnenc, Z. Trajanoski, W. Fridman and J. Galon (2005). "Effector Memory T Cells, Early Metastasis, and Survival in Colorectal Cancer." The New England Journal of Medicine 353(25): 2654-2666. 
Pai, R., A. S. Tarnawski and T. Tran (2004). "Deoxycholic acid activates beta-catenin signaling pathway and increases colon cell cancer growth and invasiveness." Mol Biol Cell 15(5): 2156-2163.

Park, M. Y., M. Y. Kim, Y. R. Seo, J. S. Kim and M. K. Sung (2016). "High-fat Diet Accelerates Intestinal Tumorigenesis Through Disrupting Intestinal Cell Membrane Integrity." J Cancer Prev 21(2): 95-103.

Peck, S. C., K. Denger, A. Burrichter, S. M. Irwin, E. P. Balskus and D. Schleheck (2019). "A glycyl radical enzyme enables hydrogen sulfide production by the human intestinal bacterium Bilophila wadsworthia." Proc Natl Acad Sci U S A 116(8): 31713176.

Peng, L., X. W. Bian, D. K. Li, C. Xu, G. M. Wang, Q. Y. Xia and Q. Xiong (2015). "Large-scale RNA-Seq Transcriptome Analysis of 4043 Cancers and 548 Normal Tissue Controls across 12 TCGA Cancer Types." Sci Rep 5: 13413.

Peters, U., S. Jiao, F. R. Schumacher, C. M. Hutter, A. K. Aragaki, J. A. Baron, S. I. Berndt, S. Bezieau, H. Brenner, K. Butterbach, B. J. Caan, P. T. Campbell, C. S. Carlson, G. Casey, A. T. Chan, J. Chang-Claude, S. J. Chanock, L. S. Chen, G. A. Coetzee, S. G. Coetzee, D. V. Conti, K. R. Curtis, D. Duggan, T. Edwards, C. S. Fuchs, S. Gallinger, E. L. Giovannucci, S. M. Gogarten, S. B. Gruber, R. W. Haile, T. A. Harrison, R. B. Hayes, B. E. Henderson, M. Hoffmeister, J. L. Hopper, T. J. Hudson, D. J. Hunter, R. D. Jackson, S. H. Jee, M. A. Jenkins, W. H. Jia, L. N. Kolonel, C. Kooperberg, S. Kury, A. Z. Lacroix, C. C. Laurie, C. A. Laurie, L. Le Marchand, M. Lemire, D. Levine, N. M. Lindor, Y. Liu, J. Ma, K. W. Makar, K. Matsuo, P. A. Newcomb, J. D. Potter, R. L. Prentice, C. Qu, T. Rohan, S. A. Rosse, R. E. Schoen, D. Seminara, M. Shrubsole, X. O. Shu, M. L. Slattery, D. Taverna, S. N. Thibodeau, C. M. Ulrich, E. White, Y. Xiang, B. W. Zanke, Y. X. Zeng, B. Zhang, W. Zheng, L. Hsu, R. Colon Cancer Family, G. the and C. Epidemiology of Colorectal Cancer (2013). "Identification of Genetic Susceptibility Loci for Colorectal Tumors in a Genome-Wide Meta-analysis." Gastroenterology 144(4): 799-807 e724.

Pfalzer, A. C., F. K. Kamanu, L. D. Parnell, A. K. Tai, Z. Liu, J. B. Mason and J. W. Crott (2016). "Interactions between the colonic transcriptome, metabolome, and microbiome in mouse models of obesity-induced intestinal cancer." Physiol Genomics 48(8): 545-553.

Rao, C. V., Y. Yang, M. V. Swamy, T. Liu, Y. Fang, R. Mahmood, M. Jhanwar-Uniyal and W. Dai (2005). "Colonic tumorigenesis in $\mathrm{BubR}^{+/-} \mathrm{Apc}^{\mathrm{Min} /+}$ compound mutant mice is linked to premature separation of sister chromatids and enhanced genomic stability." Proc Natl Acad Sci U S A 102(12): 4365-4370.

Ridlon, J. M., S. C. Harris, S. Bhowmik, D. J. Kang and P. B. Hylemon (2016). "Consequences of bile salt biotransformations by intestinal bacteria." Gut Microbes 7(1): 22-39. 
Ridlon, J. M., D. J. Kang and P. B. Hylemon (2006). "Bile salt biotransformations by human intestinal bacteria." J Lipid Res 47(2): 241-259.

Ridlon, J. M., D. J. Kang, P. B. Hylemon and J. S. Bajaj (2014). "Bile acids and the gut microbiome." Curr Opin Gastroenterol 30(3): 332-338.

Ridlon, J. M., P. G. Wolf and H. R. Gaskins (2016). "Taurocholic acid metabolism by gut microbes and colon cancer." Gut Microbes 7(3): 201-215.

Rieger, M. A., A. Parlesak, B. L. Pool-Zobel, G. Rechkemmer and C. Bode (1999). "A diet high in fat and mean but low in dietary fibre increases the genotoxic protential of 'faecal water'." Carcinogenesis 20(12): 2311-2316.

Ritari, J., J. Salojarvi, L. Lahti and W. M. de Vos (2015). "Improved taxonomic assignment of human intestinal $16 \mathrm{~S}$ rRNA sequences by a dedicated reference database." BMC Genomics 16: 1056.

Roediger, W. E. W. (1980). "Role of anaerobic bacteria in the metabolic welfare of the colonic mucosa in man." Gut 21: 793-798.

Round, J. L. and S. K. Mazmanian (2009). "The gut microbiota shapes intestinal immune responses during health and disease." Nat Rev Immunol 9(5): 313-323.

Rowan, A. J., H. Lamlum, M. Ilyas, J. Wheeler, J. Straub, A. Papadopoulou, D. Bicknell, W. F. Bodmer and I. P. M. Tomlinson (1999). "APC mutations in sporadic colorectal tumors: A mutational "hotspot" and interdependence of the "two hits"." Proc Natl Acad Sci U S A 97(7): 3352-3357.

Rubin, D. C., A. Shaker and M. S. Levin (2012). "Chronic intestinal inflammation: inflammatory bowel disease and colitis-associated colon cancer." Front Immunol 3: 107.

Sansom, O. J., J. Berger, S. M. Bishop, B. Hendrich, A. Bird and A. R. Clarke (2003). "Deficiency of Mbd2 suppresses intestinal tumorigenesis." Nature Genetics 34(2): 145147.

Sansom, O. J., V. Meniel, J. A. Wilkins, A. M. Cole, K. A. Oien, V. Marsh, T. J. Jamieson, C. Guerra, G. H. Ashton, M. Barbacid and A. R. Clarke (2006). "Loss of Apc allows phenotypic manifestation of the transforming properties of an endogenous K-ras oncogene in vivo." Proc Natl Acad Sci U S A 103(38): 14122-14127.

Scanlan, P. D., F. Shanahan, Y. Clune, J. K. Collins, G. C. O'Sullivan, M. O'Riordan, E. Holmes, Y. Wang and J. R. Marchesi (2008). "Culture-independent analysis of the gut microbiota in colorectal cancer and polyposis." Environ Microbiol 10(3): 789-798. 
Scanlan, P. D., F. Shanahan and J. R. Marchesi (2009). "Culture-independent analysis of desulfovibrios in the human distal colon of healthy, colorectal cancer and polypectomized individuals." FEMS Microbiol Ecol 69(2): 213-221.

Schmittgen, T. D. and K. J. Livak (2008). "Analyzing real-time PCR data by the comparative CT method." Nature Protocols 3(6): 1101-1108.

Scott, K. P., J. C. Martin, G. Campbell, C. D. Mayer and H. J. Flint (2006). "Wholegenome transcription profiling reveals genes up-regulated by growth on fucose in the human gut bacterium "Roseburia inulinivorans"." J Bacteriol 188(12): 4340-4349.

Segata, N., J. Izard, L. Waldron, D. Gevers, L. Miropolsky, W. S. Garrett and C. Huttenhower (2011). "Metagenomic biomarker discovery and explanation." Genome Biology 12(6): R60-R60.

Sekirov, I., S. L. Russell, L. C. Antunes and B. B. Finlay (2010). "Gut microbiota in health and disease." Physiol Rev 90(3): 859-904.

Shin, N. R., J. C. Lee, H. Y. Lee, M. S. Kim, T. W. Whon, M. S. Lee and J. W. Bae (2014). "An increase in the Akkermansia spp. population induced by metformin treatment improves glucose homeostasis in diet-induced obese mice." Gut 63(5): 727-735.

Shoemaker, A. R., K. A. Gould, C. Luongo, A. R. Moser and W. F. Dove (1997). "Studies of neoplasia in the Min mouse." Biochimica et Biophysica Acta 1332: F25-F48.

Shoemaker, A. R., A. R. Moser, C. A. Midgley, L. Clipson, M. A. Newton and W. F. Dove (1998). "A resistant genetic background leading to incomplete penetrance of intestinal neoplasia and reduced loss of heterozygosity in $A p c^{M i n} /+$ mice." Proc Natl Acad Sci U S A 95: 10826-10831.

Sinha, R., J. Ahn, J. N. Sampson, J. Shi, G. Yu, X. Xiong, R. B. Hayes and J. J. Goedert (2016). "Fecal Microbiota, Fecal Metabolome, and Colorectal Cancer Interrelations." PLoS One 11(3): e0152126.

Smith, D. L., P. Keshavan, U. Avissar, K. Ahmed and S. D. Zucker (2010). "Sodium taurocholate inhibits intestinal adenoma formation in APCMin/+ mice, potentially through activation of the farnesoid X receptor." Carcinogenesis 31(6): 1100-1109.

Sobhani, I., J. Tap, F. Roudot-Thoraval, J. P. Roperch, S. Letulle, P. Langella, G. Corthier, J. Tran Van Nhieu and J. P. Furet (2011). "Microbial dysbiosis in colorectal cancer (CRC) patients." PLoS One 6(1): e16393.

Spratlin, J. L., N. J. Serkova and S. G. Eckhardt (2009). "Clinical applications of metabolomics in oncology: a review." Clin Cancer Res 15(2): 431-440. 
Su, L., K. W. Kinzler, B. vogelstein, A. C. Preisinger, A. R. Moser, C. Luongo, K. A. Gould and W. F. Dove (1992). "Multiple Intestinal Neoplasia Caused by a Mutation in the Murine Homolog of the APC Gene." Science 256: 668-670.

Takayama, T., S. Katsuki, Y. Takahashi, M. Ohi, S. Nojiri, S. Sakamaki, J. Kato, K. Kogawa, H. Miyake and Y. Nitsu (1998). "Aberrant Crypt Foci of the Colon as Precursors of Adenoma and Cancer." The New England Journal of Medicine 339(18): 1277-1284.

Takeo T, N. N. (2011). "Reduced Glutathione Enhances Fertility of Frozen/Thawed C57BL/6 Mouse Sperm after Exposure to Methyl-Beta-Cyclodextrin." Biology of Reproduction 85: 1066-1072.

Takeyama, K., J. V. Fahy and J. A. Nadel (2001). "Relationship of Epidermal Growth Factor Receptors to Goblet Cell Production in Human Bronchi." American Journal of Respiratory and Critical Care Medicine 163: 511-516.

Thaiss, C. A., M. Levy, T. Korem, L. Dohnalova, H. Shapiro, D. A. Jaitin, E. David, D. R. Winter, M. G. BenAri, E. Tatirovsky, T. Tuganbaev, S. Federici, N. Zmora, D. Zeevi, M. Dori-Bachash, M. Pevsner-Fischer, E. Kartvelishvily, A. Brandis, A. Harmelin, O. Shibolet, Z. Halpern, K. Honda, I. Amit, E. Segal and E. Elinav (2016). "Microbiota Diurnal Rhythmicity Programs Host Transcriptome Oscillations." Cell 167: 1495-1510.

Theriot, C. M., M. J. Koenigsknecht, P. E. Carlson, Jr., G. E. Hatton, A. M. Nelson, B. Li, G. B. Huffnagle, Z. L. J and V. B. Young (2014). "Antibiotic-induced shifts in the mouse gut microbiome and metabolome increase susceptibility to Clostridium difficile infection." Nat Commun 5: 3114.

Thomas, C., R. Pellicciari, M. Pruzanski, J. Auwerx and K. Schoonjans (2008). "Targeting bile-acid signalling for metabolic diseases." Nat Rev Drug Discov 7(8): 678693.

Thune, I. and E. Lund (1996). "Physical activity and the risk of colorectal cancer in men and women." British Journal of Cancer 73: 1134-1140.

Trauner, M. and J. L. Boyer (2003). "Bile Salt Transporters: Molecular Characterization, Function, Regulation." Physiol Rev 83: 633-671.

Travaglione, S., A. Fabbri and C. Fiorentini (2008). "The Rho-activating CNF1 toxin from pathogenic E. coli: a risk factor for human cancer development?" Infect Agent Cancer 3: 4.

Tringe, S. G., C. von Mering, A. Kobayashi, A. A. Salamov, K. Chen, H. W. Chang, M. Podar, J. M. Short, E. J. Mathur, J. C. Detter, P. Bork, P. Hugenholtz and E. M. Rubin (2005). "Comparative Metagenomics of Microbial Communities." Science 308: 554-557. 
Truett, G. E., P. Heeger, R. L. Mynatt, A. A. Truett, J. A. Walker and M. L. Warman (2000). "Preparation of PCR-quality mouse genomic DNA with hot sodium hydroxide and tris (HotSHOT)." Biotechniques 29(1): 52, 54.

van Baarlen, P., F. J. Troost, S. van Hemert, C. van der Meet, W. M. de Vos, P. J. de Groot, G. J. E. J. Hooiveld, R. M. Brummer and M. Kleerebezem (2009). "Differential NF-kB pathways induction by Lactobacillus plantarum in the duodenum of healthy humans correlating with immune tolerance." Proc Natl Acad Sci U S A 106(7): 23712376.

van der Lugt, B., A. A. van Beek, S. Aalvink, B. Meijer, B. Sovran, W. P. Vermeij, R. M. C. Brandt, W. M. de Vos, H. F. J. Savelkoul, W. T. Steegenga and C. Belzer (2019). "Akkermansia muciniphila ameliorates the age-related decline in colonic mucus thickness and attenuates immune activation in accelerated aging Ercc1 (-/Delta7) mice." Immun Ageing 16: 6.

Wang, T., G. Cai, Y. Qiu, N. Fei, M. Zhang, X. Pang, W. Jia, S. Cai and L. Zhao (2012). "Structural segregation of gut microbiota between colorectal cancer patients and healthy volunteers." ISME J 6(2): 320-329.

Ward, D. M., R. Weller and M. M. Bateson (1990). "16S rRNA sequences reveal numerous uncultured microorganisms in a natural community." Nature 345: 63-65.

Weir, T. L., D. K. Manter, A. M. Sheflin, B. A. Barnett, A. L. Heuberger and E. P. Ryan (2013). "Stool microbiome and metabolome differences between colorectal cancer patients and healthy adults." PLoS One 8(8): e70803.

Weisburg, W. G., S. M. Barns, D. A. Pelletier and D. J. Lane (1991). "16S Ribosomal DNA Amplification for Phylogenetic Study." American Society for Microbiology 173(2): 697-703.

Wells, J. E., K. B. Williams, T. R. Whitehead, D. M. Heuman and P. B. Hylemon (2003). "Development and application of a polymerase chain reaction assay for the detection and enumeration of bile acid $7 \alpha$-dehydroxylating bacteria in human feces." Clinica Chimica Acta 331(1-2): 127-134.

Wikoff, W. R., A. T. Anfora, J. Liu, P. G. Schultz, S. A. Lesley, E. C. Peters and G. Siuzdak (2009). "Metabolomics analysis reveals large effects of gut microflora on mammalian blood metabolites." Proc Natl Acad Sci U S A 106(10): 3698-3703.

Wollowski, I., G. Rechkemmer and B. L. Pool-Zobel (2001). "Protective role of probiotics and prebiotics in colon cancer." Am J Clinical Nutrition 73: 451-455.

Wu, S., K. J. Rhee, E. Albesiano, S. Rabizadeh, X. Wu, H. R. Yen, D. L. Huso, F. L. Brancati, E. Wick, F. McAllister, F. Housseau, D. M. Pardoll and C. L. Sears (2009). "A 
human colonic commensal promotes colon tumorigenesis via activation of $\mathrm{T}$ helper type 17 T cell responses." Nat Med 15(9): 1016-1022.

Xia, J., I. V. Sinelnikov, B. Han and D. S. Wishart (2015). "MetaboAnalyst 3.0--making metabolomics more meaningful." Nucleic Acids Res 43(W1): W251-257.

Yazici, C., P. G. Wolf, H. Kim, T. L. Cross, K. Vermillion, T. Carroll, G. J. Augustus, E. Mutlu, L. Tussing-Humphreys, C. Braunschweig, R. M. Xicola, B. Jung, X. Llor, N. A. Ellis and H. R. Gaskins (2017). "Race-dependent association of sulfidogenic bacteria with colorectal cancer." Gut 66(11): 1983-1994.

Zackular, J. P., N. T. Baxter, K. D. Iverson, W. D. Sadler, J. F. Petrosino, G. Y. Chen and P. D. Schloss (2013). "The Gut Microbiome Modulates Colon Tumorigenesis." 4(6): $1-9$.

Zeineldin, M. and K. L. Neufeld (2013). "More than two decades of Apc modeling in rodents." Biochim Biophys Acta 1836(1): 80-89.

Zell, J. A., N. A. Ignatenko, H. F. Yerushalmi, A. Ziogas, D. G. Besselsen, E. W. Gerner and H. Anton-Culver (2007). "Risk and risk reduction involving arginine intake and meat consumption in colorectal tumorigenesis and survival." Int J Cancer 120(3): 459-468.

Zeng, H., S. Umar, B. Rust, D. Lazarova and M. Bordonaro (2019). "Secondary Bile Acids and Short Chain Fatty Acids in the Colon: A Focus on Colonic Microbiome, Cell Proliferation, Inflammation, and Cancer." Int J Mol Sci 20(5).

Zierer, J., M. A. Jackson, G. Kastenmuller, M. Mangino, T. Long, A. Telenti, R. P. Mohney, K. S. Small, J. T. Bell, C. J. Steves, A. M. Valdes, T. D. Spector and C. Menni (2018). "The fecal metabolome as a functional readout of the gut microbiome." Nat Genet 50(6): 790-795. 


\section{VITA}

Jacob (Jake) Moskowitz was born and raised in suburban Baltimore, Maryland. Jake enjoyed spending his earliest years outside on the soccer field, forging new paths through the local forests, and taking care of a variety of pets. He was eager to pursue his academic interests in the sciences, and so departed Baltimore to complete a Bachelor's degree in biology at Case Western Reserve University in Cleveland, Ohio. During his time at Case Western, Jake developed a passion for research as an undergraduate assistant in the labs of Dr. Kristian Baker and Dr. Peter Harte, where he studied nonsense-mediated decay of mRNA and the impact of epigenetic modifications on aging in Drosophila, respectively. He also discovered a passion for foreign language and culture during his time in Cleveland, which led him to pursue minors in both Spanish and German language, and an unforgettable study abroad experience in Edinburgh, Scotland. While in Edinburgh, Jake discovered an opportunity to care for a variety of farm species, which spurred a strong interest in veterinary medicine.

In 2010, Jake was accepted to attend the University of Missouri, College of Veterinary Medicine to pursue his newfound passion for veterinary medicine. He always maintained his strong interest in the biomedical sciences, which led him to engage in a number of research opportunities during his "spare" time in veterinary school. Under the mentorship of Drs. Chris Baines, Yuksel Agca, and Craig Franklin, Jake explored a number of topics including mitochondrial involvement in breast cancer, cryo-recovery of ovarian tissue function, and finally interactions between the gut microbiome and epigenetics. From these experiences, Jake knew that he wanted to pursue post-DVM opportunities that would allow him to combine his passions for medicine and research. 
After completing an elective rotation with MU's Comparative Medicine Program, Jake decided that pursuing a residency in laboratory animal medicine with the CMP was the perfect way to incorporate all of his interests, and was fortunate to join the program after completing his DVM in 2014. As Jake continued to hone his research interests, he maintained a strong interest in the gut microbiome and hoped to investigate its role in cancer. To that end, he joined the laboratory of Dr. James Amos-Landgraf within the Department of Veterinary Pathobiology to explore interactions between host genetics and the gut microbiota in a mouse model of colorectal cancer. The exciting results of this work are described in detail in the body of this dissertation.

Jake continues to have a strong interest in research, pathology, and medicine, and looks forward to the next stages of his burgeoning career. He is incredibly grateful for the invaluable guidance he has received from his mentors, without whom he would not be where he is today. Jake also expresses his gratitude to his family and friends who have supported his long-winded academic pursuits without question. Moving forward, Jake plans to further his expertise in a translational research setting studying the role of the gut microbiome in intestinal diseases. He hopes to remain in the academy, where he can also pursue his passion for teaching the next generation of young scientists and veterinarians. Ultimately, Jake hopes to use his research career to bridge human and veterinary medicine, and to have a positive impact on biomedical research that benefits the health of all species. 